\title{
SINGLE-WELL TRACER PUSH-PULL METHOD DEVELOPMENT FOR SUBSURFACE PROCESS CHARACTERIZATION
}

Early-time tracer injection-flowback test for stimulated fracture characterization, numerical simulation uses and efficiency for flow and solute transport

Dissertation

-to acquire the doctoral degree in mathematics and natural science

"Doctor rerum naturalium"

-at the Georg-August-Universität Göttingen in the doctoral program Geoscience at Georg-August University School of Science (GAUSS)

Submitted by

Shyamal Karmakar

from Rangamati, Bangladesh

Göttingen, 2016 


\section{Betreuungsausschuss:}

Professor Dr. Martin Sauter, GZG - Abteilung Angewandte Geologie, Universität Göttingen Dr. Iulia Ghergut, GZG - Abteilung Angewandte Geologie, Universität Göttingen

\section{Mitglieder der Prüfungskommission}

\section{Referent:}

Professor Dr. Martin Sauter, GZG - Abteilung Angewandte Geologie, Universität Göttingen

\section{Koreferent:}

Dr. Iulia Ghergut, GZG - Abteilung Angewandte Geologie, Universität Göttingen

Professor Dr. Günter Buntebarth, Instituts für Geophysik, Technische Universität Clausthal

\section{weitere Mitglieder der Prüfungskommission:}

Professor Dr.-Ing. Thomas Ptak-Fix, GZG - Abteilung Angewandte Geologie, Universität Göttingen

Dr. Reinhard Jung, Jung Geotherm, Isernhagen, Hannover

Dr. Ulrich Maier, GZG - Abteilung Angewandte Geologie, Universität Göttingen

Tag der mündlichen Prüfung: 15.06.2016 
To my Mother

and to my TEACHERS 


\section{Summary}

Geological inherent knowledge, hydraulic test and geophysical methods can estimate most of the stimulated georeservoir properties. The transport effective parameters such as fracture aperture and effective porosity cannot estimate by using these methods. The in-situ methods, the inter-well test or single-well test, are sensitive to transport effective parameters. Transport effective parameters determine geo reservoir's efficiency, sustainability or lifetime. The inter-well test needs more than one well which is not typical to install during early stage of geo-reservoir development to avoid too much investment before a proven use is confirmed. Hence, single-well test design for a specific sensitivity regime from 'early' to 'very-late' pull signal is rather practical for transport effective parameter estimation. Moreover, a typical single-well test that design for 'mid' to 'late-time' signal also loaded with many sensitive parameters. Secondly, tracer test design, and parameter sensitivity estimation depends on numerical simulation reliability. Finite element and finite difference code based different numerical method shows significant improvement toward this parameter inversion and test design.

The use of single-well (SW) short-term tracer signals to characterize stimulated fractures at the Groß-Schönebeck EGS pilot site is studied in chapter 2, part 1. Short-time tracer flowback signals suffer from ambiguity in fracture parameter inversion from measured single-tracer signals. This ambiguity arises commonly due to a certain degree of interdependence between parameters such as fracture porosity, fracture thickness, fracture dispersivity. This ambiguity can, to some extent, be overcome by (a) combining different sources of information, and/or (b) using different types of tracers, such as conservative tracer pairs with different diffusivities, or tracer pairs with contrasting sorptivity on target surfaces. Fracture height is likely to be controlled by lithostratigraphy while fracture length can be determined from hydraulic monitoring (pressure signals). Since the flowback rate is known during an individual-fracture test, the unknown parameters to be inferred from tracer tests are (i) transport-effective aperture in a water fracture or (ii) fracture thickness and porosity for a gel-proppant fracture. Tracers with different sorptivity on proppant coatings and matrix rock surfaces for gel-proppant fractures and tracers with contrasting-diffusivity or -sorptivity for a water fracture were considered. This simulation study has produced two significant results: (1) water fracture aperture can be effectively evaluated based on early-time tracer signals of a conservative tracer; and (2) by using the combination of matrix sorptive and proppant sorptive tracers, it is possible to estimate fracture thickness and porosity in gel proppant fractures from a single test. The injection and flowback of a small fluid volume, and thus little dilution of the injected tracers, has three practical advantages: (1) there is no need to 
inject large tracer quantities; (2) one does not have to wait for the tails of the test signals; and (3) the field and laboratory monitoring of the tracer signals does not have to be conducted for ultralow tracer concentrations, which is known to be a major challenge, with the highly-mineralized and especially high organic content fluids typically encountered in many sedimentary basement georeservoir. Additionally, it requires only a very small chaser injection volume (about half of fracture volume).

Short-term flowback signals from injection-flowback tracer test face a certain degree of ambiguity in fracture parameter inversion from the measured signal of a single tracer. To improve the earlytime characterization of induced fractures, of either gel-proppant or waterfrac, we recommend using tracers of contrasting sorptivity to rock surfaces, and to proppant coatings where applicable. The application that described in early time flowback tracer test study article at chapter 2, part 1. However, the tracer was not exhaustively demonstrating its complete range of uses for stimulated georeservoir. Sorptive tracer either on proppant or on a matrix that used for stimulated fracture characterization has raised the question about the range of sorptive tracer to produce for an effective tracer test. For the purposes, a lower sorptive tracer than its minimum necessary was suggested and a sensitivity improvement factor (ratio between sorptive tracer signal changes to conservative tracer signals changes, s/c) approximately equal to $\sqrt{ }(1+0.7 \times$ sorption coefficient, $\kappa)$ is formulated. One needs to note that the higher the tracer's retardation, the lower is its fracture invasion, and consequently a poorer capability for characterizing the fracture as a whole. In principle, this could be compensated by increasing the chaser volume (i.e., by injecting sorptive tracers earlier than conservative tracers).

Modeling flow and solute transport become a state of the art for a set of engineering and hydrogeological applications. For hydrogeological modeling, a number of numerical software is available as commercial code as well as many research initiatives is emerging to develop a new one. This section (Chapter 3) of the thesis attempts to develop solute transport module in fracture using COMSOL. FEFLOW software with it discrete feature element (e.g. fracture) module it can simulate fully couple process for flow, solute, and heat simulation. For the study of early time, tracer flowback signal, the flow, and solute transport process coupling in fracture-matrix domain is studied using tetrahedral mesh. To compare the consistency of numerical result with spatial and temporal discretization as well as in different numerical approach, a same numerical model set up in COMSOL. Qualitative comparison of the between the codes reveals that dispersivity tensor application can cause a minor variation in the tracer breakthrough in single-well tracer flowback 
simulation. The result is compared in terms similarities and capturing the spikes of injection and flowback in early flow back tracer test.

A set of well-established software, frequently used for modeling flow and transport in geological reservoirs, is tested and compared (MODFLOW/MT3DMS, FEFLOW, COMSOL Multiphysics and $\mathrm{DuMu}^{\mathrm{x}}$ ). Those modeling tools are based on different numerical discretization schemes i.e. finite differences, finite volumes and finite element methods. The influence of dispersivity, which is directly related to the numerical modeling, is investigated in parametric studies and results are compared with analytical approximations. At the same time, relative errors are studied in a complex field scale example. For 1D and 2D cases all three tested modeling software show good agreement with the analytical solutions. By refining the grid discretization all four software packages get an improvement in accuracy. It is shown for the $2 \mathrm{D}$ problem that COMSOL Multiphysics needs a finer mesh to produce the same accuracy as FEFLOW and DuMux ${ }^{\mathrm{x}}$ For transport simulations in forced gradient, where a commonly expected dispersion or higher value occurs, the finite element software FEFLOW is the best choice. From this comparative study, it is revealed that under forced gradient conditions, finite element codes COMSOL and FEFLOW show a higher accuracy with respect to the analytical approximation for a certain range of dispersivity than $\mathrm{DuMu}^{\mathrm{x}}$ and MODFLOW/MT3DMS. Comparing simulation time and code parallelization, FEFLOW performs better than COMSOL. Computational time is lowest for finite difference software MODFLOW/MT3DMS for a small number of mesh elements $(\sim$ less than 12800 elements). For large meshes (12800 elements or higher) finite element software FEFLOW performs better. Nevertheless, the study showed that improving the numerical performance by optimizing discretization methods, solvers and parallelization methods still remain a crucial field of research. 


\section{Table of Contents}

Single-well tracer push-pull method development for subsurface process characterization

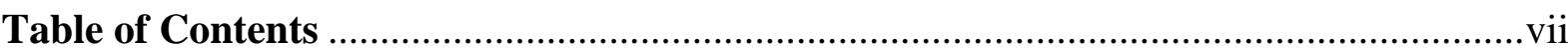

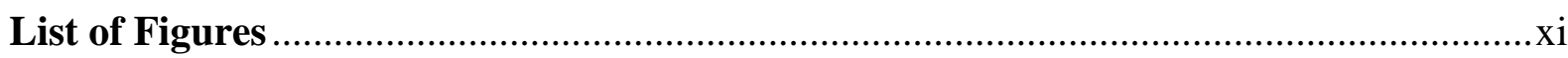

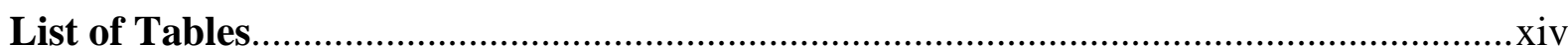

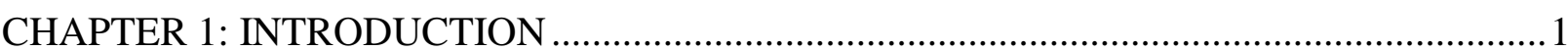

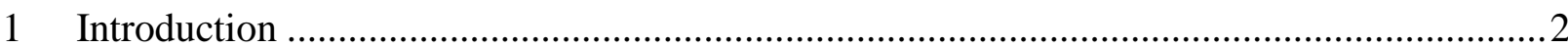

1.1 Renewable energy sources and geothermal energy.............................................2

1.2 Significance of fracture characterization for stimulated geo-reservoir ..........................2

1.3 Single well tracer test and tracer flowback for fracture characterization....................... 3

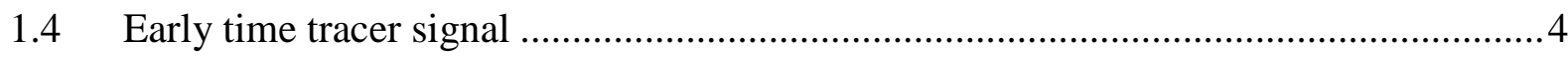

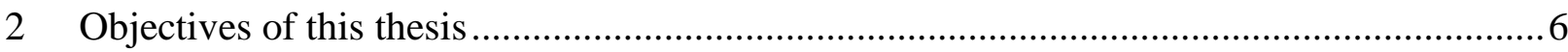

2.1 Single well tracer push-pull/injection-flowback test: dispersion in porous media and fractured porous media; Chapter 2- Part 1, Part 2 and Part 3 ................................................. 7

2.1.1 Part 1: Early-time tracer signal for fracture thickness, fracture porosity, and dispersivity in gel-proppant fracture and dispersivity, fracture aperture in water fracture $\ldots . .8$

2.1.2 Part 2: Early time tracer injection-flowback test: injection duration- 'Tpush' and 'injection rate' effect on the parameter sensitivity .....

2.1.3 Part 3: Multiple fracture and single fracture systems for sorption-matrix diffusion

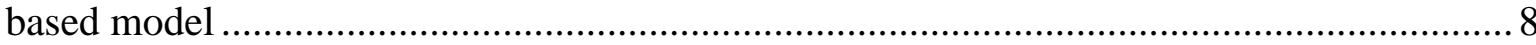

2.2 Benchmark study on flow and solute transport; Chapter 3- Part 1 and Part 2 .............. 9

2.2.1 Part 1: Spatial and temporal discretization sensitivity to single fracture simulation using finite element code FEFLOW and COMSOL- a benchmark study ...........................9

2.2.2 Part 2: Benchmark Study On Flow and Solute Transport in Geological

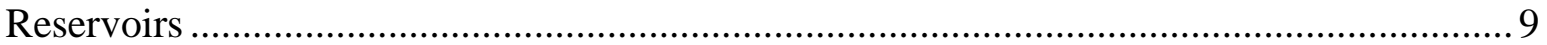

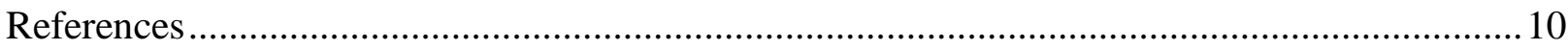

CHAPTER 2-PART 1: Early-flowback tracer signals for fracture characterization in an EGS developed in deep crystalline and sedimentary formations: a parametric study ....................... 14

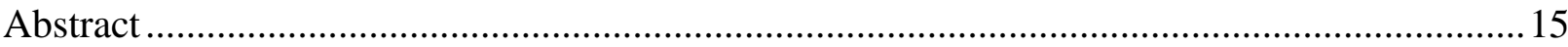

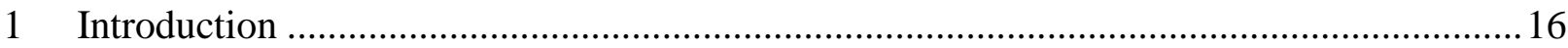

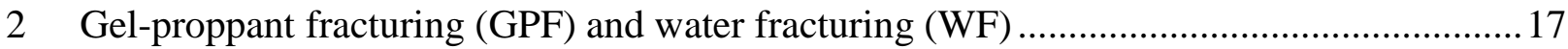

3 Model setup, transport processes, and simulation parameters for tracer tests.................... 18

4 Spatial discretization and hydraulic treatment of injection-flowback tests .......................22

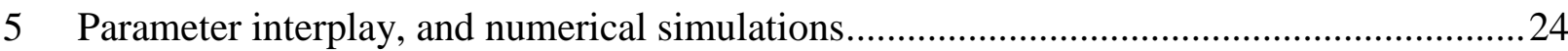

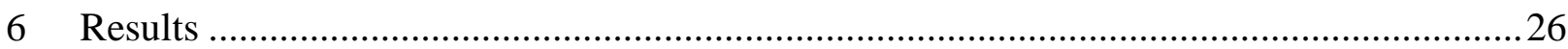

6.1 Conservative-tracer signals during early flowback ..............................................26 
6.2 MST signals during early flowback

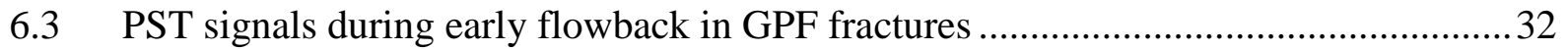

7 Discussion, and recommendations for future tracer tests ...............................................34

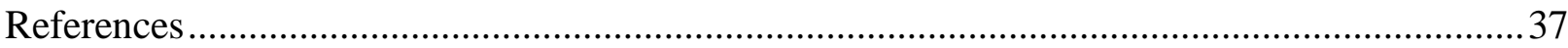

CHAPTER 2-PART 2: EGS in sedimentary basins: sensitivity of early-flowback tracer signals to

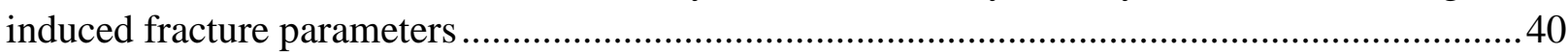

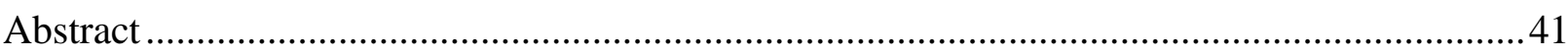

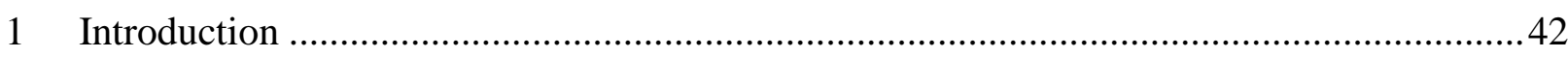

2 Gel proppant fracture and water fracture simulation parameters ..................................43

3 Conservative and sorptive tracer test in gel proppant fracture ........................................4 43

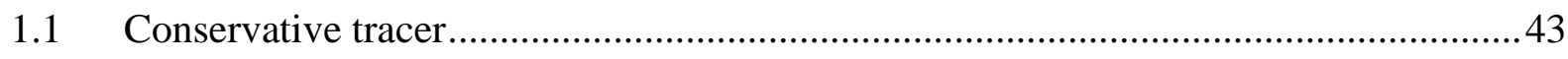

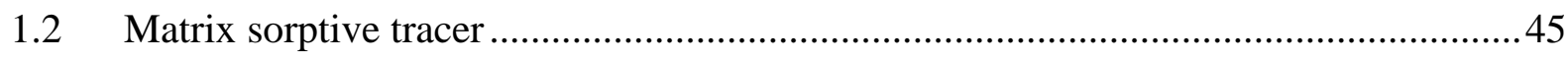

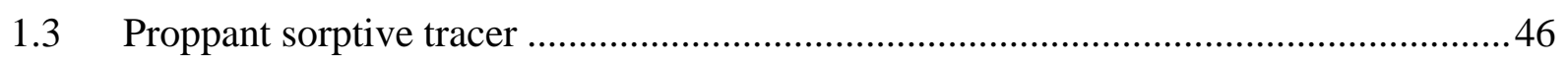

2 Conservative and sorptive tracer test in water fracture ............................................. 48

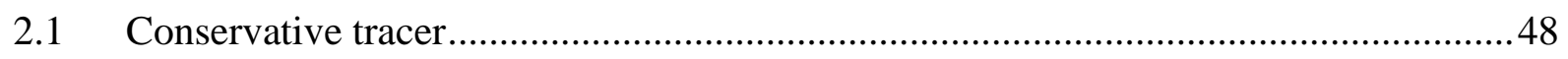

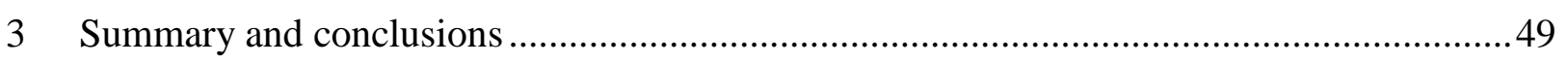

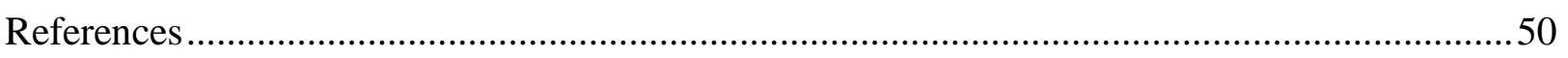

CHAPTER 2-PART 3: Early time flowback tracer test for stimulated crystalline-georeservoir of

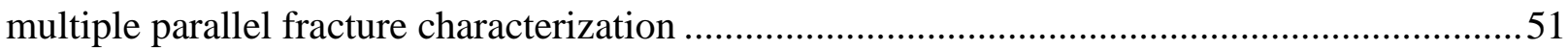

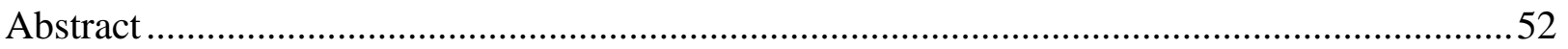

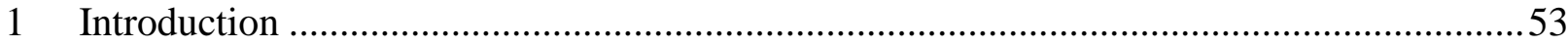

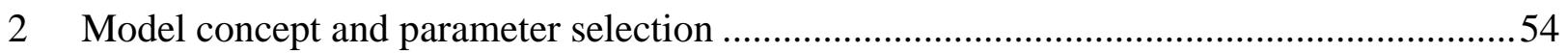

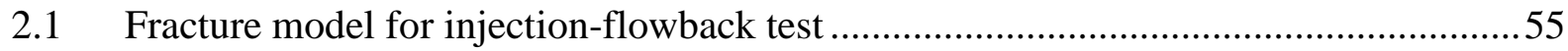

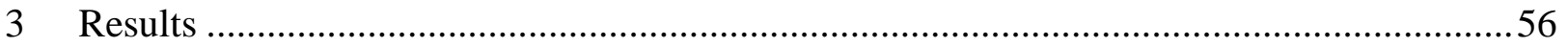

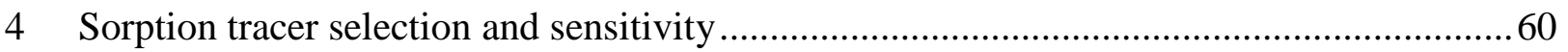

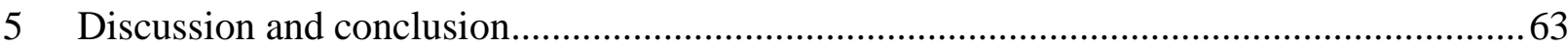

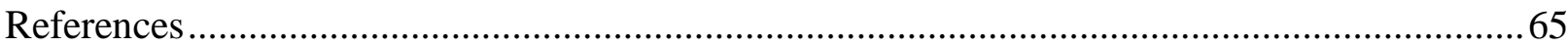

CHAPTER 3-PART 1: Single-well tracer injection-flowback test simulation in fractured georeservoir using finite element code FEFLOW and COMSOL- a benchmark study ..............66

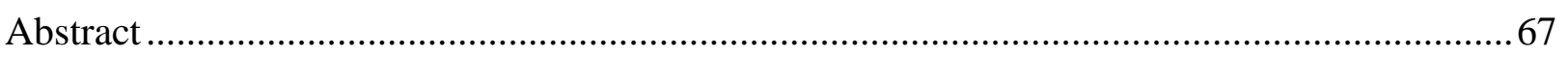

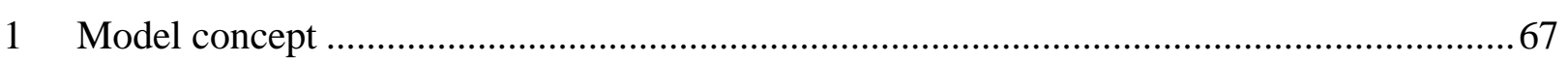

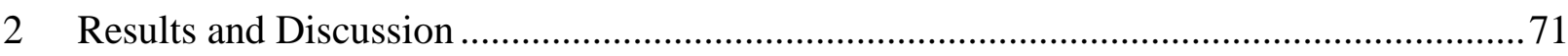

2.1 Spatial and Temporal discretization effect on FEFLOW single fracture SW injection

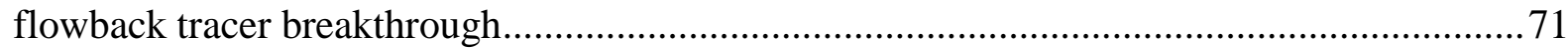

2.2 Tracer flowback signals in COMSOL and FEFLOW from gel-proppant fracture and

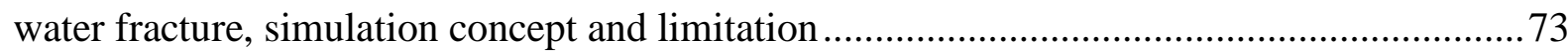


CHAPTER 3-Part 2: A set of benchmark studies on flow and solute transport in geological

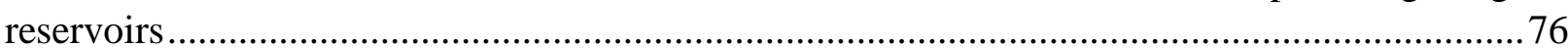

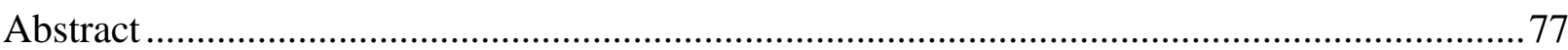

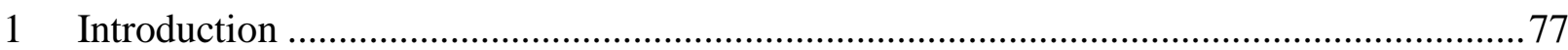

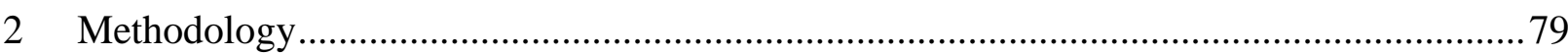

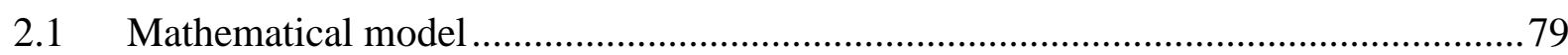

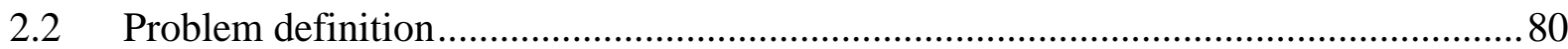

2.2.1 Problem 1: 1D - Solute tracer transport for steady state flow with a forced head

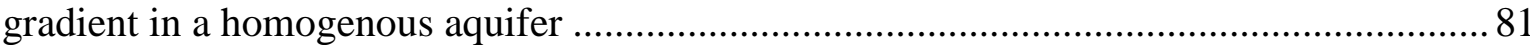

2.2.2 Problem 2: 2D-Solute transport in a confined homogenous aquifer from a forced

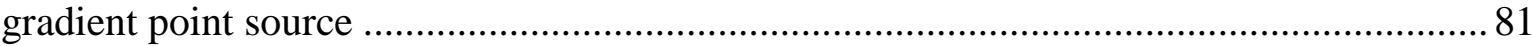

2.2.3 Problem 3: 3D- Solute transport for confined homogeneous multi-layered forced

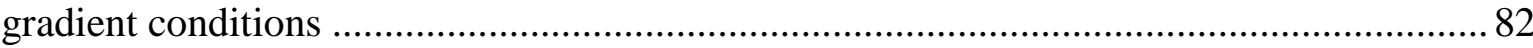

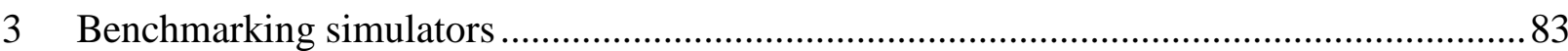

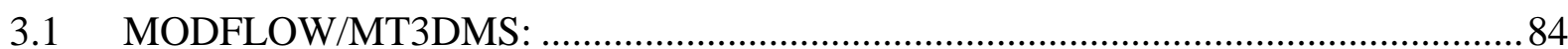

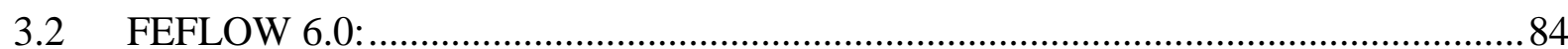

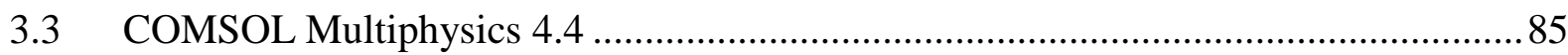

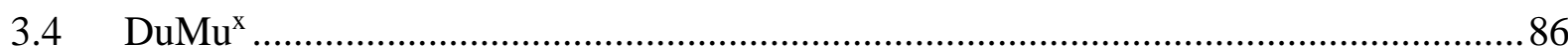

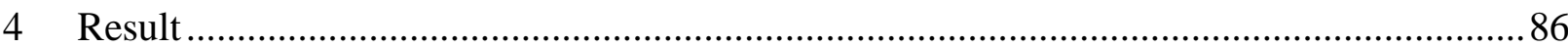

4.1 Problem 1: 1D - Solute transport in a homogeneous aquifer with a natural gradient ..86

4.2 Problem 2: 2D-Solute transport in forced gradient homogeneous aquifer.................... 88

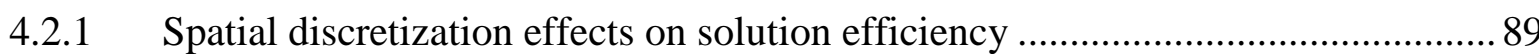

4.3 Problem 3: 3D -Flow and solute transport simulation in a layered georeservoir..........90

4.4 Simulation time (CPU time) of single processor and parallelization ...........................94

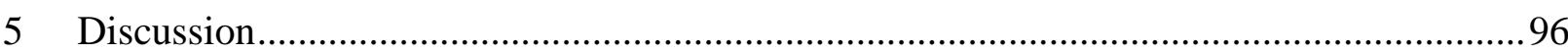

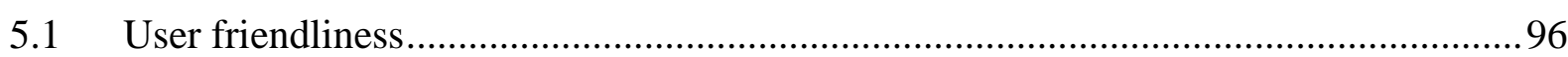

5.2 Solute transport simulation efficiency of the benchmark problems ............................97

5.3 Model implementation, simulation time and resource use efficiency and discretization .

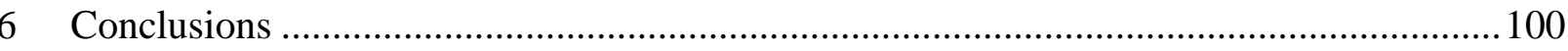

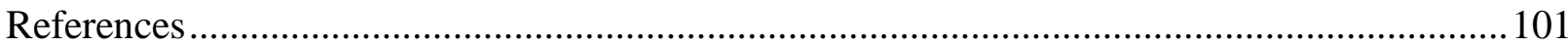

CHAPTER 4: GENERAL DISCUSSION, CONCLUSIONS, AND FUTURE WORK............103

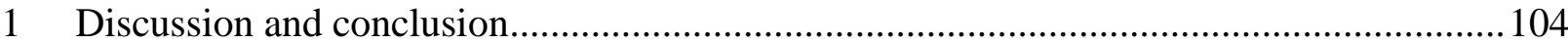

1.1 Single well tracer injection-flowback/withdrawal test-early-time tracer signal study 104

1.2 Tracer selection for early time tracer injection-flowback test................................... 107

1.3 Benchmark study for efficient numerical method selection and code development... 108 
2.1 Early time solute tracer push-pull test for dispersion estimation in aquifer......

2.2 Heat tracer uses for pulse injection flowback test-design and parameter estimation.. 110

2.3 Solute tracer pulses injection flowback test-design and parameter estimation in shale/gas

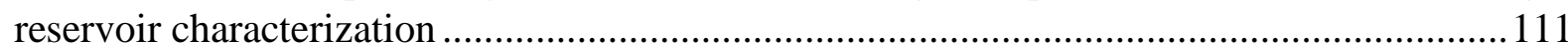

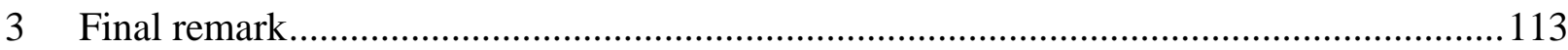

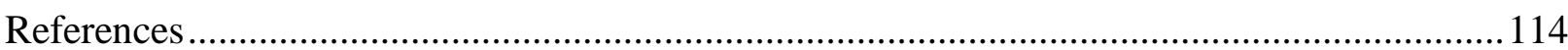

Appendix 1-Early flowback tracer signal for fracture characterization using proppant sorptive, matrix sorptive and conservative tracers.

Appendix 2a: Single-well tracer injection-flowback test simulation in fractured georeservoir in FEFLOW and COMSOL

Appendix 2b: A set of benchmark studies for flow and solute transport in geo-reservoir

List of Publications

Conference proceedings, poster, and oral presentation

Curriculum Vitae

Acknowledgement 


\section{List of Figures}

Figure 2.3.1: 3D model domain and hydrogeological parameter distribution (after Blöcher et al., 2010)

Figure 2.4.1: Fluid and tracer injection-flowback in GPF and WF treatments. The transition from constant injection to constant flowback was approximated as linear (dashed-line segment); its duration is enlarged for better recognition. In the simulations, the transition spans only 1/60 of Tpush.

23

Figure 2.6.1: Simulated signals of conservative tracers during GPF flowback: the effect of dispersion processes (expressed by eight different values of longitudinal dispersivity $\alpha L$, as indicated by signal labels, in meters), shown for two different values of GPF thickness (solid line: $12 \mathrm{~mm}$, dashed-dotted line: 20mm), with a fixed value of GPF porosity (45\%).

Figure 2.6.2: Simulated signals of conservative tracers during GPF flowback: the effect of dispersion processes (expressed by eight different values of longitudinal dispersivity $\alpha L$, as indicated by signal labels, in meters), shown for three different values of GPF porosity (solid line: 30\%, dashed line: 45\%, dasheddotted line: 60\%), with a fixed value of GPF thickness (12mm). 28

Figure 2.6.3: Simulated signals of conservative tracers during WF flowback: the distinct effects of fracture aperture (with values represented by different shadings as shown by legend) and of longitudinal dispersivity (solid line: $7 m$, dash-dotted line: $5 m$ ).

Figure 2.6.4: Simulated signals of various MST (characterized by different Kd values) during GPF flowback, at a fixed value of GPF thickness (12 mm) and proppant-packing porosity $30 \%)$. Signals are labeled by the retardation factor $R$, instead of $K d$ values the signal of a conservative (non-sorptive, $K d=0, R=1$ ) tracer is shown for comparison. 30

Figure 2.6.5: Simulated signals of two MST during GPF flowback: the effect of GPF thickness (values are indicated as signal labels, in $\mathrm{mm}$ ), with a fixed value of proppant-packing porosity (45\%), shown for two tracer species, a less sorptive one (dimensionless $\kappa=0.9$ ), and a more sorptive one (dimensionless $\kappa=1.52$ ).

Figure 2.6.6: Simulated signals for one MST (characterized by dimensionless $k=1.5$ ) during GPF flowback: the effect of GPF thickness (values are indicated as signal labels, in $\mathrm{mm}$ ), with two values of GPF porosity (solid line: 30\%, broken line: 60\%)

Figure 2.6.7: Simulated signals for one PST (characterized by dimensionless $k=40$ ) during GPF flowback: the effects of GPF thickness and of GPF porosity (solid lines: 35\%, broken lines: 55\%) illustrate parameter interplay.

Figure 2.6.8: Simulated signals for one PST (characterized by dimensionless $k=40$ ) during GPF flowback: the effect of GPF thickness (values are indicated in $\mathrm{mm}$ ), and the effect of GPF porosity (gray tones, from light: $30 \%$, to dark: 60\%), illustrating the increase of porosity sensitivity with increasing thickness....... 34 Figure 2.2.1: Conservative tracer flowback signals from different dispersivity and fracture thickness: broken line- $4 \mathrm{~mm}$, solid line- $16 \mathrm{~mm}$ .44

Figure 2.2.2: Conservative tracer flowback signals from different dispersivity and fracture porosity......44 
Figure 2.2.3: MST ( $k=1.5)$ tracer concentrations resulting from a GPF treatment with fracture porosity $55 \%$

Figure 2.2.4: PST $(k=40)$ flowback concentration for porosity (por) 35\% and 55\% for fracture thickness ( $t F$ ) in gel-proppant fracture.

Figure 2.2.5: Tracer injection duration effect on flowback proppant sorptive tracer signals for different fracture porosity.

Figure 2.2.6: Tracer injection duration effect on flowback proppant sorptive tracer signals for different fracture thickness.

Figure 2.2.7: Conservative tracer flowback signals for different fracture aperture (af) and dispersion length $(d L)$ in WF treatment.

Figure 2.3.1: Conceptual model of HDR type EGS formation and injection-flowback well. The red box indicates fracture and matrix domain for simulation that pertaining 1/8 of a fracture volume from equally spaced fractures. 56

Figure 2.3.2: Weak matrix sorption tracer (MSTs) sensitivity in different fracture length for hydraulically stimulated fracture in HDR type EGS. It shows that very weak sorptive or conservative tracer $R=1-1.5$, matrix porosity $3 \%, k=0-0.01$ is sensitive to the fracture length while a big aperture $(1 \mathrm{~mm}-2 \mathrm{~mm})$ is created.

Figure 2.3.3: Strong MST (matrix sorption tracer) sensitivity in different fracture length for hydraulically stimulated fracture in HDR type EGS, matrix porosity $1 \%$ for a fracture aperture $2 \mathrm{~mm}$. Sensitive sorptive tracer range is $k-0.5-1.5$.

Figure 2.3.4: Medium range MSTs sensitivity in different fracture length for hydraulically stimulated fracture in HDR type EGS, matrix porosity $1 \%$ for a fracture aperture $1 \mathrm{~mm}$. Sensitive sorptive tracer range is $k-0.5-1.5$.

Figure 2.3.5: Effect of higher injection rate/volume, which exceed the fracture volume, for the high MST ( $k$ 5-100, matrix porosity-3\%). It shows that strong MSTs show no clear trend with the fracture length.60 Figure 2.3.6: Simulated signals of multiple PST and MST (characterized by different Kd values) during GPF flowback, at a fixed value of GPF thickness $(12 \mathrm{~mm})$ and proppant-packing porosity (30\%). 61 Figure 2.3.7: Simulated signals of multiple PST during GPF flowback, at a different value of GPF and proppant-packing porosity (30\% and $60 \%$ ). 62 Figure 2.3.8: Simulated signals of multiple PST (characterized by different Kd values) during GPF flowback, at a fixed value of GPF thickness $(12 \mathrm{~mm})$ and proppant-packing porosity (40\%). Signals are labeled by the retardation factor $k$ ( $k=K d \times$ proppant density).

Figure 3.1.1: 3D model domain and hydrogeological parameter distribution. The rectangular mesh shown here is used in FEFLOW simulation. COMSOL simulation is done in the triangular mesh. 69 Figure 3.1.2: Spatial and temporal discretization effect on FEFLOW numerical solution single fracture tracer flowback concentrations. 
Figure 3.1.3: Tracer spreading inside the fracture and matrix during at 1 day while flowback start at 0.5 days for conservative tracer-3a (from left), low matrix sorptive tracer-3b and high MSTs- 3c............... 73 Figure 3.1.4a-b: Tracer concentration (a) and Hydraulic head (b) from different fracture porosity for injection-flowback benchmark study in single fracture-matrix domain using PDEs module in software package COMSOL Multiphysics. 74

Figure 3.2.1: Problem 1:1D model domain assuming a free flow boundary at right end and higher gradient at left end with a constant point contaminant source.

Figure 3.2.2: Problem 2: 2D domain assuming a radial symmetry of flow and solute at the injection point of solute located the middle of the domain. Hence, half of the domain is taken into account for the numerical simulation, with the injection well and point source applied at the middle point of the lower border of the domain and the rest of the lower border constrained as no-flow boundary. .82 Figure 3.2.3: 3D model domain, showing the rectangular mesh and the permeability and porosity distribution over the layers. Left side points: injection points; right side points: pumping well. .83 Figure 3.2.4: Problem 1, tracer breakthrough from various solvers with dispersivities $5 \mathrm{~m}$ and $0.7 \mathrm{~m} . . . .87$ Figure 3.2.5: The relative difference between the numerical and analytical solutions within the simulated range of dispersivity values.

Figure 3.2.6: Time-concentration curve for two different dispersivity value $0.7 \mathrm{~m}$ and $5 \mathrm{~m}$ simulated in MODFLOW-MT3DMS, FEFLOW, COMSOL and DUMU ${ }^{x}$ for problem 2D and analytical solution from Gelhar and Collins (1971)...

Figure 3.2.7: The relative difference of the numerical solution from the analytical solution for different dispersion value for benchmark problem 2: 2D.

Figure 3.2.8: Spatial discretization sensitivity on the solution accuracy convergence in different simulators for a standard dispersivity $5 \mathrm{~m}$. .90 Figure 3.2.9a-c: 3D model tracer concentration from numerical simulation using MODFLOW, FEFLOW, COMSOL Multiphysics and DuMux respectively at three different layers a) top layer b) middle layer c) bottom layer with a dispersivity value $5 \mathrm{~m}$ .92 Figure 3.2.10a-c: Relative difference of tracer concentration for different dispersivity from concentration curve of MODFLOW/MT3DMS at three different layers a) top layer b) middle layer c) bottom layer......94 Figure 4.1: Dispersion value sensitivity of Schroth et al. (2001) single-well tracer push-pull test pull signal. 


\section{List of Tables}

Table 1: Values of matrix and fracture parameters in WF and GPF model

Table 2: Solute tracer uses and sensitivity with different EGS georeservoirs

Table 3: Simulation time: Computational time in the simulation computer for the problem 2-2D domain and refined mesh.

Table 4: Simulation time: Computational time in the simulation computer for the problem 3-3D domain and refined mesh.

Table 5: Early time tracer push-pull test uses in fracture characterization 
CHAPTER 1: INTRODUCTION 


\section{Introduction}

\subsection{Renewable energy sources and geothermal energy}

Renewable energies feature as a high impact issue on the global energy agenda with a strong perception of reduced uncertainty (WEC, 2016). A green energy source, such as the wind, solar and hydropower energy with a relatively small environmental footprint, suffer from temporality or seasonality challenged the sustainability of aspired 'decarbonized societies' energy supply. Geothermal energy, as the largest renewable energy resources (WEA, 2000; IPCC, 2011), with an estimated technical potential of up to 5000 EJ per year, can potentially supplement up to $8.3 \%$ of the total world electricity (WEC, 2016). Geothermal energy, belonging to green energy sources with the least carbon footprint; it can resolve the riddle of temporal variations of energy through complementing base load energy supply. Petro-thermal resources are commonly occurring in the crystalline basement throughout the world, and lowenthalpy hydrothermal resource occurs in the sedimentary basement at a depth of $3 \mathrm{~km}-7 \mathrm{~km}$ (Breede et al. 2013). Geothermal resource needs to ensure a certain degree of permeability between the wells and a certain amount of contact surface area known as heat exchange area (Ghergut 2011) to provide production which can be achieved by stimulating fractures through hydraulic fracturing.

\subsection{Significance of fracture characterization for stimulated geo-reservoir}

The engineered geothermal system (EGS) is the promising technology that enriched with a many experimentations and learning as well as realizations (Jung 2013). The success and longterm viability of any geothermal energy extraction scheme based on heat transfer from hot rock to circulating fluids essentially depends on upon the contact surface between the host porous rock and the active fracture network. For an industrial scale, viable geothermal resource requires a $\sim 501 / \mathrm{s}$ pumping rate from a volume of rock to be accessed by the fracture system has to be in the order of $0.2 \mathrm{~km}^{3}$ (Rybach 2004) with a temperature of $>160{ }^{\circ} \mathrm{C}$ for a period of 25-30 years (Stober et al, 2014, Jung 2013, Breede 2013). The success of stimulation can estimate using appropriate characterization techniques. Ptak and Teutsch (1994) and others (Ghergut et al., 2011) agreed that the size and properties of fracture surface cannot unambiguously be determined by hydraulic or geophysical methods nor from the short-term temperature signals. An early characterization, using first drilled well, will undoubtedly reduce the cost of geothermal resource development. Therefore, the use of the single-well for characterization is rather practical and financially more attractive. 
The estimation of the fracture geometry is one of the most difficult technical challenges in hydraulic fracturing technology (Zhang et al., 2010). Albeit, tracer method cannot achieve characterization goal while working "stand-alone" rather needs a concurrent effort using a geophysical method and/or hydrological method. Inter-well or single-well tracer test gives an opportunity for in-situ appraisal of subsurface process parameters like 'effective porosity' and 'interface/exchange area density'. The inter-well tests are more appealing as it able to investigate flow-path properties over long distances, encompassing large reservoir volumes. In contrary, the SWPP test enables flow reversibility observations which are advantageous for the evaluation of time-dependent processes (e.g. Nordqvist and Gustafsson, 2002; Ghergut et al., 2012).

\subsection{Single well tracer test and tracer flowback for fracture characterization}

Single-well tracer push-pull (SWPP) method developed to quantify fluid phase saturation in two-phase systems using reactive/partitioning (PTTs) in oil reservoir engineering tests (Tomich et al. 1973, Sheely, 1978). Thereafter, it became a standard practice in a wide range of uses covering flow field characterization (advection velocities, and/or dispersivities, cf. Bachmat et al. 1984, Leap and Kaplan 1988), to characterizing everything else except flow fields, matrix diffusion (Kocabas and Horne 1987, Haggerty et al. 2001, Pruess and Doughty 2010, Jung and Pruess 2012, Ghergut et al 2013b); in-situ reaction (assuming AD, matrix diffusion, etc. negligible or can be calibrated away) (Istok et al. 1997, Haggerty et al. 1998, Snodgrass and Kitanidis 1998, Schroth et al. 2001, Lee et al. 2010). Some authors have discussed the use of SWPP in the dominion of georeservoir characterization (e.g. Carrera et al. 1998, Snodgrass and Kitanides 1998, Ghergut et al. 2007, 2011, Herfort and Sauter 2003, Herfort et al. 2003). A useful literature overview on various experiences made with SWPP is given by Neretnieks (2007), with a focus on applications in the realm of contaminant hydrology as well as geological storage. Single well tracer test is known only for characteristic tracer signal during the flowback. However, the 'flowback' term appeared in in this thesis for this method in many instances always not synonymous with 'pull' or 'withdrawal'. The terms 'injection-flowback' (SWIF) are used in the context of SW tests pertain different meaning and context from the 'backflow' describe in more details in Chapter 2, part 1 (Karmakar et al. 2015a). With an objective to control the interaction of time dependent process on the target surface or volume, SWPP or SWIF consists a/multiple 'shut-in' period or no shut-in period before pull phase. In deep geothermal wells, assuming a non-existence of a production pump (at a depth of several hundred meters), in the case of sufficient pressure build up, injection-flowback provides an 
inexpensive method for SW tracer tests aimed at quantifying fluid and heat transport in the target formation.

\subsection{Early time tracer signal}

SWPP or SWIF method suffers from the limitations (non-uniqueness of interpreted parameters) (Haggerty et al., 1998; Schroth et al., 2001; Novakowski et al.,1998;) caused by parameter 'interplay', necessitate characteristic types of tracer development and test design. The way to reduce the ambiguity from SWPP signal is to reduce/enhance the sensitive parameters. This specific goal can only achieve through identification a sensitivity regime. The parameter interplay in pull signal has initiated an innovative tracer test design so that it can reduce the sensitive parameter. An effort toward this goal, Ghergut et al. (2013b) has identified four characteristic regimes in pull signals viz. 'early-time,' 'mid-time' or 'late time' or 'very late time' in single-planer fracture model. They have identified ten sensitive parameters in the initial-boundary value problems (IBVP) in the transport PDE for SWPP test.

$$
\begin{aligned}
& \frac{\partial C}{\partial t}+\frac{Q}{2 \pi B_{e f f}} \frac{\partial C}{r \partial r}-\frac{\alpha|Q|}{2 \pi B_{e f f}} \frac{\partial^{2} C}{r \partial r^{2}}-\left.\frac{\varphi_{m} D_{m}}{b} \frac{\partial C}{r \partial y}\right|^{y=a}=0 \\
& \frac{\partial C_{m}}{\partial t}-D_{m} \frac{\delta^{2} C_{m}}{\delta y^{2}}-D_{m} \frac{\delta^{2} C_{m}}{\delta r^{2}}=0 \ldots \ldots \ldots \ldots \ldots \ldots \ldots \ldots \ldots \ldots \ldots \ldots \ldots \ldots \ldots
\end{aligned}
$$

It includes two fracture geometrical parameters (fracture aperture 'b'- relevant with $B_{\text {eff }}$ and fracture spacing ' $a$ ' in parallel fracture system - relevant to y), five hydrogeological properties (matrix porosity, matrix diffusion coefficients, longitudinal dispersivity within fracture, 'aquifer' thickness, hydraulic diffusivity-all related to dispersion tensor $D_{m}$ in fracture and matrix), and three SWPP test design variables, pull phase duration, injection and extraction rates or volumes (Ghergut et al., 2013a), where many of them not sensitive or possible to ignore during an 'early-time' SWPP test (Karmakar et al 2015a, 2015b). However, the fracture parameter estimation potential from this kind of single-well tracer test in stimulated georeservoir is not apprehended before as first recognized by Ghergut et al. (2013b). Furthermore, though traditionally dispersion seldom recognizes as a single well push-pull sensitive parameter, Behrens et al. (2009) and Ghergut et al. (2011) identified that 'dispersion' is not fully insensitive to SWPP tracer method. This thesis includes a novel application (sorption) for fracture parameter estimation also discussed non-traditional push-pull parameter such as dispersion estimation too. 
A conceptual model for stimulated fracture parameter estimation using single-well tracer method founded on the lesson learned from several tracer studies in Northern and Southeast German sedimentary and crystalline basement. One-eighth of fracture-matrix volume assumed suffice to model due to considering the symmetry of fracture axis perpendicular to the injection well with a planar fracture (Ghergut et al., 2013b) for parallel-fracture systems. The partial differential equation of linear flow and transport equation are solved the IBVPs numerically by using a commercial finite element software, FEFLOW 6.0 (Diersch 2011) analyzing the output (i.e., the simulated tracer signals) regarding sensitivity to target parameters and of parameter interplay, as applicable). Though all hydrogeological parameters are of distributed (local) type, and their values may change with time by virtue of coupled THMC processes (as induced by SWPP-forced hydraulic and thermal gradients), implying a virtually infinite number of degrees of freedom, one global value for each parameter is assumed during the simulation study, i.e. a spatially homogeneous system whose properties do not change with time (see also Ghergut et al. 2006, 2011, 2013a). Multiple tracers of different sorptivity and diffusivity are considered for early time tracer flowback test following the idea of Maloszewski and Zuber (1992) in a single fracture model.

Surface sorptive tracer: The EGS evaluation report (USDOE 2008) has recommended on the needs of measuring rockfluid interface areas in geothermal systems, stating that "reliable tracers that can measure and/or monitor the surface area responsible for rock-fluid heat and mass exchange do not exist”. Again, its Glossary enlisted two separate tracer definitions: a mere "tracer" being used to determine flow paths and velocities, and a "smart tracer" being needed for determining "the surface area contacted by the tracer". The sorption of solutes from the flowing fluid to the reservoir rock being a process that directly involves the fluid-rock interface, it seems that sorptive tracers can provide the answer to the cited USDOE challenge (Ghergut et al 2012). Rose et al (2011) investigated how the use of "quantum dot tracers with controllable surface sorption characteristics", and with "low matrix diffusivity" within "singlewell tracer testing methodologies should result in significant advances in the interrogation of surface area in enhanced geothermal reservoirs". Indeed, unlike matrix diffusion (cf. Carrera et al. 1998, Haggerty et al. 2001), tracer sorption appears as a robust, easily-quantifiable process, whose modeling is much less intricate than that of matrix diffusion, and also much less dependent on various theoretical assumptions regarding void-space structure. The tracer that used in stimulated georeservoir for flow-path tracings using single-well test or inter-well test or a combination of both, mostly as forced gradient flow condition during a field test are 
ranged from spiked water molecule (Tritium) to organo-molecules (e.g. fluorescein dye, naphthalene di-sulfonate etc.) also assumed to be stable in very different pressure and temperature situation in georeservoir (e.g. Ghergut et al 2016). A great deal of time and resource has been invested over decades to develop a stable tracer group that will not dissociated, react, precipitated in highly variable pressure and temperature situation in georeservoir and revealed a significant success (Rose et al 2011, 2012, Dean et al 2015) in georeservoir application. However, use of different surface sorptive tracer in georeservoir characterization rather new to be reported in literature or case studies. Furthermore, Rose et al. (2011) has described a new tracer group 'nano-colloidal CdSe', a semi-conductive material based fluoresce tracer, to reveal reservoir parameters. Colloidal nanocrystal 'quantum dots' are small crystallites of semiconductors ( 1 to $\sim 20 \mathrm{~nm}$ ) that is composed of a few hundred to several thousands of atoms. Due to their reduced spatial dimensions, nanometer-sized semiconductors display unique size and shape-related electronic and optical properties as a result of quantum size effects and strongly confined excitons (Alivisatos, 1996; Efros et al, 2003). Moreover, using a surface sensitive coating (i.e. proppant sorptive tracer and matrix sorptive tracer) on the quantum dot tracer will bring a new generation tracer which can be detected in the visible to near infrared range. This tracer development initiative would influence use of tracer for georeservoir characterization scheme greatly. The anticipation from surface sensitive tracer in EGS characterization becomes evident from the study in this thesis (chapter 2).

Numerical technique to solve flow and solute transport problem has a significant improvement in last two decades. Moreover, with the increase of computation capacity, the numerical simulation in standard laptop computer is also possible. The finite element software, FEFLOW was used in the most of the study (chapter 2 and part of chapter 3). In tracer test design, test result interpretation for single-well tracer test and simulation result reliability and efficiency are some major issues that apportioned and discussed in this thesis in Chapter 3.

\section{Objectives of this thesis}

1. Development of SWPP method is to estimate-

i) fracture parameter of stimulated geo-reservoirs of sedimentary where fracture porosity, fracture thickness

ii) fracture aperture, dispersivity inside the fracture of crystalline geo-reservoir

iii) fracture length of parallel fracture of HDR types EGS 
2. The reliability and efficiency of numerical simulators result for flow and solute transport in-

i) fractured geo-reservoir comparing SWPP test tracer signal arises from the simulation of finite element method code, FEFLOW, and COMSOL.

ii) geo-reservoir of simple to layer formation tracer signal in different flow regime from the simulation in MODFLOW/MT3DMS, FEFLOW, COMSOL, and $\mathrm{DuMu}^{\mathrm{x}}$.

This thesis consists of two chapters where each chapter is subdivided into parts based on applications and scenarios to satisfy these two objectives. The goal at number one is explicitly demonstrated and studied in chapter 2 , for three target parameter of stimulated fracture, viz. fracture thickness, fracture porosity of stimulated fracture of sedimentary formation and fracture aperture and dispersivity in crystalline formation. Furthermore, fracture length has found as a sensitive parameter in parallel fracture EGS of HDR types. This result is precious for characterization of fracture, eventually sustainability and monitoring of this type georeservoir. The second objective is discussed and studied in chapter 3 numerical dispersion and simulation result efficiency are the primary parameter to achieve. The section below outlines the chapters of the thesis with a very brief overview of contents, methods and expected results.

\subsection{Single well tracer push-pull/injection-flowback test: dispersion in porous media and fractured porous media; Chapter 2- Part 1, Part 2 and Part 3}

'Early time' tracer single-well test using different sensitivity regime for sorptive tracer and conservative tracer, can overcome the parameter interplay in gel-proppant fracture flowback tracer signals. The anticipations of tracer test of single-well configuration that describe by Ghergut et al. (2011), advective and non-advective role of fracture aperture will interplay and cause ambiguous tracer signal from different parameter. However, the scale of interaction will vary with the 'time' and 'space'. Following this analogy, it would be effective to design diffusion-sorption separating tracer not necessarily based on only 'late time' signals (Haggerty et al. 2001, Ghergut et al., 2011), but 'early' to 'mid time' signal. Moreover, injection duration (Tpush) as described by Ghergut et al. (2011) for the fractured formation and Carrera's (1998) matrix, and injection rate effect on early-time signal would be interesting to observe. 
2.1.1 Part 1: Early-time tracer signal for fracture thickness, fracture porosity, and dispersivity in gel-proppant fracture and dispersivity, fracture aperture in water fracture

Artificial-fracture design, and fracture characterization during or after stimulation treatment is an important aspect, both in gel-proppant fracture (GPF) or water fracture (WF) type EGS. Hydraulic fracturing (EGS) in sedimentary formation usually supported by gel-proppant to stabilize the fracture size and volume after the stimulation hence can have a certain porosity which also varied with reservoir type and proppant-gel operation during stimulation. Stimulated fracture in crystalline formation pertained relatively long thin fracture and assumed to have $100 \%$ porosity. This study includes a use of specific surface sensitive tracer (proppant sorptive tracers and matrix sorptive tracers) using small injection volume and sampling at an early flowback time for the tracer concentration to evaluate fracture porosity, fracture thickness in the gel-proppant fracture. At the same time, it also discussed the use of conservative tracer for fracture aperture and dispersion in fracture estimation in water fracture of stimulated georeservoir.

\subsubsection{Part 2: Early time tracer injection-flowback test: injection duration- 'Tpush' and 'injection rate' effect on the parameter sensitivity}

Use of sorptive and conservative tracer in the realm of early time tracer injection-flowback test discussed in chapter 2, part 1 in details. In the line of this application, it is important to understand the characteristic 'injection duration' i.e. volume of injection as well as 'injection rate' for this early time tracer flowback test. Injection duration or 'Tpush' is regarded as the major influencing and deterministic parameter in 'late time tracer signal' (Haggerty et al. 2000, Ghergut et al 2013b). The specific importance behind that tracer diffusivity, i.e. the material properties of a tracer, is not compatible or sensitive to the target process/parameter here for the 'short /early-time' test.

\subsubsection{Part 3: Multiple fracture and single fracture systems for sorption-matrix diffusion based model}

This section is dealing with a finite number of discrete parallel-fracture systems, in homogeneous crystalline formation with an identical aperture and spacing with an unknown fracture length in HDR type EGS. During early time tracer injection flowback, injection duration/volume does not allow to flood the matrix also cut the interact with 'fracture spacing.' Hence, multiple fractures with equivalent spacing each remains as discrete fracture during the 
test, however, tracer signal produces a distinctive signal during flowback to evaluate fracture length for a limited range of tracers.

\subsection{Benchmark study on flow and solute transport; Chapter 3- Part 1 and Part 2}

Numerical simulator modeling subsurface solute transport is difficult-more so than modeling heads and flows. The classical governing equation does not always adequately represent what it seen at the field scale, hence commonly used numerical models are solving the wrong equation (Konikow 2011) as well as no single numerical method sufficiently works well for all conditions. The accuracy and efficiency of the numerical solution to the solute-transport equation are more sensitive to the numerical method chosen than for typical groundwater-flow problems. However, numerical errors can be kept within acceptable limits if sufficient computational effort is expended. In chapter 3, this thesis includes a benchmark study that accounts result from group projects using different numerical method and codes to solve the flow and solute transport problem in georeservoir. To compare the efficiency and reliability of numerical code, this study was conducted for flow and solute transport for four conditions, viz., 3D-singlewell injection flowback/withdrawal in single fracture georeservoir condition (part 1), and 1D -natural gradient, 2D-forced gradient in homogeneous aquifer, 3D-forced gradient in layered georeservoir (part 2)

\subsubsection{Part 1: Spatial and temporal discretization sensitivity to single fracture simulation using finite element code FEFLOW and COMSOL- a benchmark study}

The single well early flowback tracer study conducted in chapter 2 FEFLOW simulation results for fluid flow and solute transport using tetrahedral mesh with adaptive refinement approach has produced consistent result (which was used in throughout Chapter 2) using a relatively small number of elements hence it required low computation cost. In this part of the thesis, time step refinement and spatial discretization were studied, and simulation results were compared with COMSOL 'double continuum' approach result which is using triangular element and refined time step refined result for single fracture.

\subsubsection{Part 2: Benchmark Study On Flow and Solute Transport in Geological Reservoirs}

Benchmarking numerical software for fluid flow and solute transport is a state of the art for decades. Flow and solute transport code 'finite difference' 'finite element' and 'finite volume' method that used in MODFLOW/MT3DMS, FEFLOW and COMSOL, and DUMU ${ }^{\mathrm{x}}$, respectively, simulation result for flow and solute transport were compared for different geometrical complexity (1D, 2D and 3D) and different flow conditions. The software packages are compared on solution accuracy, efficiency, i.e. time and computer resources needed, user friendliness and financial cost. From this study, it was 
understood that numerical code was capable of capturing the tracer behavior in common dispersion condition. FEFLOW numerical code is efficient to simulate flow and solute transport in porous media and fractured media with a relatively small number of mesh elements.

Early time tracer signal based single well injection flowback test showed a vast improvement of SWIW method. This pulse injection and flowback based method have shown that if the flowback pressure builds up is sufficiently enough to expect a flowback from georeservoir of sedimentary formation or crystalline formation, parameter determination from tracer signal is evident with a small number of sampling. And benchmark study on flow and solute transport show that for a simple model numerical simulation result is efficient and for complex, problem numerical simulation results need to be verified with well tested numerical code.

\section{References}

Alivisatos, A.P. 1996. Semiconductor Clusters, Nanocrystals, and Quantum Dots, Science 271, 933-937.

Bachmat Y., Behrens H. et al. 1984. Entwicklung von Einbohrlochtechniken zur quantitativen Grundwassererkundung. GSF-Berichte, R 369, München.

Behrens H, Ghergut I, Sauter M and Licha T., 2009, Tracer properties, and spiking results - from geothermal reservoirs, Proceedings, 34th Work- shop on Geothermal Reservoir Engineering, Stanford University, Stanford, CA, SGP-TR-187.

Breede K, Dzebisashvili K, Liu X, Falcone G, 2013, A systematic review of enhanced (or engineered) geothermal systems: past, present and future. Geothermal Energy. doi:10.1186/2195-9706-1-4.

Carrera J, Sanchez-Vila X, Benet I, Medina A, Galarza G, Guimera J. 1998, On matrix diffusion: formulations, solution methods and qualitative effects, Hydrogeology J, 6, 178-190

Diersch, H.-J., G., 2011, FEFLOW 6.0: finite element subsurface flow and transport simulation system. Reference manual. DHI-WASY Ltd., Berlin, Germany, 292 pp

Efros, A.L., Lockwood, D.J., Tsybeskov, L., Eds. 2003, Semiconductor Nanocrystals: From Basic Principles to Applications, Springer: New York.

Ghergut, I., McDermott, C.I., Herfort, M., Sauter, M. and Kolditz, O. 2006, Reducing ambiguity in frac- tured-porous media characterization using single-well tracer tests. IAHS Publications, $304,17-24$.

Ghergut1, I. Sauter M., Behrens H. 1, Licha T., McDermott C.I., Herfort M., Rose P., Zimmermann G., Orzol, J., Jung, R., Huenges, E. Kolditz, O., Lodemann, M., Fischer, S. Wittig, U. Güthoff, F. Kühr, M. 2007, Tracer tests evaluating hydraulic stimulation at deep geothermal reservoirs in Germany, Proceedings, Thirty-Second Workshop on Geothermal Reservoir Engineering Stanford University, Stanford, California, January 22-24, 2007 SGP-TR-183 
Ghergut, I., Behrens, H., Maier, F., Karmakar, S., Sauter, M., 2011, A note about "heat exchange areas" as a target parameter for SWIW tracer tests. In: Proceedings of the 36th Workshop on Geothermal Reservoir Engineering, Stanford University, Stanford, California. January 31 February 2, 2011, SGP-TR-191.

Ghergut, I., Behrens, H., Licha, T., Maier, F., Nottebohm, M., Schaffer, M., Ptak, T., Sauter, M., 2012. Single-well and inter-well dual-tracer test design for quantifying phase volumes and interface areas. In: Proceedings of the 37th Workshop on Geothermal Reservoir Engineering, Stanford University, Stanford, California, January30-February 1, 2012, SGP-TR-19

Ghergut, I., Behrens, H., Sauter, M., 2013a. Can Peclet numbers depend on tracer species? going beyond SW test insensitivity to advection or equilibrium exchange. In: Proceedings 38th Workshop on Geothermal Reservoir Engineering, Stanford Univ. (CA), SGP-TR-198, 326335 .

Ghergut, I., Behrens, H., Sauter, M., 2013b. Single-well tracer push-pull test sensitivity to fracture aperture and spacing. In: Proceedings 38th Workshop on Geothermal Reservoir Engineering, Stanford Univ. (CA), SGP-TR-198, 295-308.

Haggerty R, Schroth M H, Istok J D, 1998. A simplified method of "Push-pull" test data analysis for determining in situ reaction rate coefficients, Ground Water, 36(2), 313-324.

Haggerty, R., McKenna, S.A., Meigs, L.C., 2000. On the late-time behavior of tracer test breakthrough curves. Water Resources Research 36(12), 3467-3479.

Herfort M, Sauter M 2003, Investigation of matrix diffusion in deep hot-dry-rock reservoirs using SWIW tracer tests. In: Krasny, Hrkal, Bruthans (eds), Groundwater in Fractured Rocks, Prague, 257-258

Herfort, M., Ghergut, I. and Sauter, M. 2003, Investigation of Matrix Diffusion in Deep Hot-DryRock Reservoirs Using Single-Well Injection-Withdrawal Tracer Tests. Eos Transactions, 84(46), AGU Fall Meeting Supplement, Abstract H51H-02.

Istok J D, Humphrey M D, Schroth M H, Hyman M R, O'Reilly K. T. 1997, Single-Well, "PushPull” Test for In Situ Determination of Microbial Activities. Ground Water, 35(4), 619-631

IPCC, 2011, Summary for Policymakers. In: IPCC Special Report on Renewable Energy Sources and Climate Change Mitigation, Cambridge University Press, Cambridge, United Kingdom and New York, NY, USA.

Jung, Y. and K. Pruess 2012, "A closed-form analytical solution for thermal single-well injectionwithdrawal tests," Water Resour. Res., 48, W03504, doi:10.1029/2011WR010979.

Jung, R., 2013. EGS-Goodbye or Back to the Future, Chapter 5. In: Bunger, A.P., McLennan, J., Jeffrey, R. (Eds.), Effective and Sustainable Hydraulic Fracturing. InTechOpen, pp. 95-121, http://dx.doi.org/10.5772/56458.

Karmakar, S., Ghergut, I., Sauter, M., 2015a. Early-flowback tracer signals to induced-fracture characterization in crystalline and sedimentary formation-a parametric study. Geothermics, in press, doi:10.1016/j.geothermics.2015.08.007. 
Karmakar, S., Ghergut, I., Sauter, M., 2015b. EGS in sedimentary basins: sensitivity of earlyflowback tracer signals to induced-fracture parameters. Energy Procedia 76, 223-229. doi:10.1016/j.egypro.2015.07.906

Kocabas I, Horne R N 1987, Analysis of Injection-Backflow Tracer Tests in Fractured Geothermal Reservoirs. Procs 12th Workshop on Geothermal Reservoir Engineering, Stanford University, SGP-TR-109

Konikow, L.F. 2011, The Secret to Successful Solute-Transport Modeling, Ground Water 49(2), $144-159$.

Lee J. H., Dolan M., Field J., Istok J. 2010. Monitoring Bio-augmentation with Single-Well PushPull Tests in Sediment Systems Contaminated with Trichloroethene. Environ. Sci. Technol., 44(3), 1085-1092.

Leap D I, Kaplan P G 1988, A single-well tracing method for estimating regional advective velocity in a confined aquifer: theory and preliminary laboratory verification. Water Research, 23(7), 993-998

Maloszewski P. and Zuber, A. 1992, On the calibration and validation of mathematical models for the interpretation of tracer experiments in groundwater Advances in Water Resources 15, 47 62

Neretnieks I. 2007, Single-well injection-withdrawal tests (SWIW) in fractured rock. Some aspects on interpretation. SKB Report R-07-54 (Swedish Nuclear Fuel and Waste Management Co., Stockholm).

Nordqvist, R., Gustafsson, E., 2002. Single-well injection-withdrawal tests (SWIW). Literature review and scoping calculations for homogeneous crystalline bedrock conditions. Swedish Nuclear Fuel and Waste Management Co., Stockholm (Sweden).

Novakowski K S, Lapcevic P, Voralek J W, 1998. A note on a method for measuring the transport properties of a formation using a single well, Water Resources Research, 34(5), 1351-1356.

Pruess, K., Doughty, C., 2010. Thermal single-well injection-withdrawal tracer tests for determining fracture-matrix heat transfer area. In: Proceedings of the 35th Workshop on Geothermal Reservoir Engineering, Stanford University, Stanford, CA, USA, February 1-3, 2010, SGP-TR-188.

Ptak T, Teutsch G 1994, A comparison of investigation methods for the prediction of flow and transport in highly heterogeneous formations, Dracos \& Stauffer (eds), Transport and Reactive Processes in Aquifers, Balkema, Rotterdam, 157-16

Rybach, L. 2004, EGS-the state of art. Tagungband der 15. Fachtagung der Schweizerischen Vereinigung für Geothermie, Stimulierte Geothermische Systeme, 7p Basel.

Schroth, M.H., Istok, J.D., Haggerty, R., 2001. In situ evaluation of solute retardation using singlewell push-pull tests. Advances in Water Resources, 24, 105-117.

Sheely, C.Q., 1978. Description of Field Tests to Determine Residual Oil Saturation by SingleWell Tracer Method, SPE, Journal of Petroleum technology, 194-202 
Snodgrass M F, Kitanides P K, 1998. A method to infer in situ reaction rates from push-pull experiments, experiments, Ground Water 36(4), 645-650

Stober I, Fritzer T., Obst, K., and Schulz R., 2014, Deep Geothermal Energy, Application possibility in Germany, Edited by Bruchmann U., BMWi, Department IIC 6, the Federal Ministry of Economic Affairs, pp 82. www.bmwi.de

Tomich J F, Dalton R L Jr, Deans H A, Shallenberger L K 1973, Single-Well Tracer Method to Measure Residual Oil Saturation. Journal of Petroleum Technology / Transactions, 255, 211218.

USDOE 2008, An Evaluation of Enhanced Geothermal System Technology, Geothermal technologies program, US Department of Energy, 37pp

WEC, 2016, World Energy Council 2016 World Energy Issues Monitor 2016, A climate of innovation - responding to the commodity price storm, pp143. www.worldenergy.org

WEA, 2000. World energy assessment: energy and the challenge of sustainability. Prepared by UNDP, UN-DESA and the World Energy Council United Nations Development Programme, New York. 508pp.

Zhang, GM, Liu H, Zhang J. Wu HA and Wang XX, 2010, Three dimensional finite element simulation and parametric study form horizontal well hydraulic fracture. J. Petrol. Sci. Eng. 72 (3-4), 310-317. http://dx.doi.org/10.1016/j.petrol.2010.03.032. 
CHAPTER 2-PART 1: Early-flowback tracer signals for fracture characterization in an EGS developed in deep crystalline and sedimentary formations: a parametric study

Shyamal Karmakar*, Julia Ghergut and Martin Sauter

Citation:

Karmakar, S., Ghergut, J., Sauter, M., 2015. Early-flowback tracer signals for fracture characterization in an EGS developed in deep crystalline and sedimentary formations: a parametric study, Geothermics, in press, doi:10.1016/j.geothermics.2015.08.007

Geoscience Centre of the University of Göttingen, Department of Applied Geology, Goldschmidtstraße 3, 37077 Göttingen, Germany

*Corresponding author: shyamal.karmakar@geo.uni-goettingen.de 


\begin{abstract}
Artificial-fracture design and fracture characterization is a central aspect of many Enhanced Geothermal System (EGS) projects. The use of single well (SW) short-term tracer signals to characterize fractures at the Groß-Schönebeck EGS pilot site is explored in this paper. A certain degree of parameter interdependence in short-term flowback signals leads to ambiguity in fracture parameter inversion from measured single-tracer signals. This ambiguity can, to some extent, be overcome by (a) combining different sources of information, and/or (b) using different types of tracers, such as conservative tracer pairs with different diffusivities, or tracer pairs with contrasting sorptivities on target surfaces. Fracture height is likely to be controlled by lithostratigraphy while fracture length can be determined from hydraulic monitoring (pressure signals). Since the flowback rate is known during an individual-fracture test, the unknown parameters to be inferred from tracer tests are (i) transport-effective aperture in a water fracture or (ii) fracture thickness and porosity for a gel-proppant fracture. Tracers with different sorptivity on proppant coatings and matrix rock surfaces for gel-proppant fractures, and tracers with contrasting-diffusivity or -sorptivity for a water fracture were considered. An advantage of this approach is that it requires only a very small chaser injection volume (about half of fracture volume).
\end{abstract}

Keywords: Geothermal, EGS, solute tracer, sorptive tracer, diffusive tracer, water fracture, gelproppant fracture, single-well tests, injection-flowback tests 


\section{Introduction}

Artificial-fracture design, and fracture characterization during or after stimulation treatment is an important aspect of many Enhanced Geothermal System (EGS) projects, both in gelproppant fracture (GPF) or water fracture (WF) type stimulation. Well tests (pumping tests) and geophysical methods can provide valuable information on aquifer/reservoir properties, e.g., hydraulic conductivity, anisotropy, and average fracture aperture, including heterogeneity and boundary conditions (Singhal and Gupta, 2010). Analogous to ordinary porous media, transport mechanisms in fractured rock also follow common processes such as advection, hydrodynamic dispersion, molecular diffusion, rock-water interaction, tracer decay and retardation. Pressure transient tests and geophysical methods cannot be used to infer the transport-effective values of parameters such as effective porosity and fluid-rock interface area. Tracer testing is a standard method of determining mass transport within a subsurface reservoir and can be a valuable tool in the design and management of production and injection operations (Pruess and Bodvarsson, 1984; Horne, 1985; Pruess, 2002; Rose et al., 2004; Nottebohm et al., 2010).

Single-well (SW) 'injection-flowback' or 'push-pull' tracer methods are attractive for a number of reasons (Ghergut et al., 2013a). Late-time signals from SW as well as inter-well tracer tests are used for parameter estimation for porous-fractured media; this is based on the existence of different parameter sensitivity regimes with increasing residence time (Guimerà and Carrera, 1997; Haggerty et al., 2000; Ghergut et al., 2013b). In geothermal applications, SW tracer methods have been deployed to estimate fractured reservoir parameters using thermosensitive tracers (Nottebohm et al., 2010), sorptive tracers (Rose et al., 2012), and ion-exchange tracers (Dean et al., 2015). Mid-late tracer signals from SW push-pull tests have been considered mainly for the purpose of inflow profiling in multi-zone EGS reservoirs in the NE-German basin (Ghergut et al., 2014). Potential of short-term SW tracer signals for fracture characterization has remained unexplored so far. Also there is debate on how many different tracers should be 'used' per fracture (to 'use' meaning 'to sacrifice', since it will not be possible to use the same tracer later to quantify reservoir-scale properties, if the tested borehole becomes a production well). In addition, there are open questions on the expected improvements to parameter sensitivity, and desired transport/reactivity properties that multiple tracers need to have (especially in terms of diffusion, sorption, and decay). Further recommendations regarding early-sampling frequency as a trade-off between 'too much effort' and 'too sparse information', especially for the case of gel-proppant fractures, where early flowback sampling 
is likely to pose greater difficulties, are desirable. Test schedule, including the frequency and amount of tracer injection during fracturing operations, is also of great importance. So far, no 'effort-versus-benefit' analysis has been undertaken in a focused manner, leaving issues like the above as a matter of speculation. This paper explores and outlines the benefits of early flowback sampling, and of using more than one tracer per fracture. The goal is to provide greater insight to fracture characterization in an EGS developed in deep crystalline and/or sedimentary formations.

The terms 'injection-flowback' or 'huff-puff', 'injection-withdrawal' or 'push-pull' (all used in the context of SW tests) are not synonymous. 'Flowback' or 'puff' refers to fluid flowing back from the well, without a production pump, by virtue of sufficient pressure buildup during the prior injection stage. 'Withdrawal' or 'pull' refer to fluid produced from the well by means of a production pump. In deep geothermal wells, installing a production pump (at a depth of several hundred-meters) is technically non-trivial, and rather expensive. Installing a production pump for short-term testing purposes, in a borehole intended to serve as future injection well is often considered 'too much of a luxury', especially since the downhole pump emplacement would need to be performed before knowing whether the whole exercise was worthwhile. Whether sufficient pressure buildup can be attained during the prior injection stage to ensure significant flowback volume, depends on injection rate and duration, and on hydraulic properties of the formation. Thus, whenever feasible, injection-flowback provides an inexpensive opportunity for SW tracer tests aimed at quantifying fluid and heat transport in the target formation.

\section{Gel-proppant fracturing (GPF) and water fracturing (WF)}

In EGS, natural or forced fluid circulation takes place through fracture networks in crystalline rocks of low matrix porosity, or through void-space networks in sedimentary rocks characterized by higher porosities and permeabilities (Huenges, 2010). In single-fracture EGS, fluid flows mainly through the fracture. Fluid transfer between the fracture and matrix greatly depends on their permeability contrast or, more generally, their transmissivity ratio (and effective thickness values).

In GPF-based EGS developed in sedimentary formations, the stimulated fractures are kept open by using proppants. The purpose of the gel added to the injectate is to promote cohesion, so that the proppant can travel to the tip of the stimulated fracture. On the other hand, WF-based fractures generally can be developed in low-permeability or 'tight' rocks by injecting a large amount of water into a small targeted wellbore region isolated with packers. WF stimulations 
generate relatively longer fractures whose widths are smaller than those produced by chemical or GPF stimulation treatments (Mayerhofer et al., 1997; Mayerhofer and Meehan, 1998; Rose et al., 2010). The success of WF treatments mostly depends on the self-propping and shear displacement potentials of the formation. The lengths of the WF can be a few hundreds of meters and apertures are of the order of $1 \mathrm{~mm}$ (Huenges, 2010). GPF treatments were used to stimulate reservoirs with cross-linked gels in conjunction with proppants (mostly sand) of a specific mesh size. Fractures produced by the GPF process have relatively short lengths (about $50-100 \mathrm{~m})$ and apertures of up to $10 \mathrm{~mm}$.

Most of the fractured rock aquifers show double-porosity character. The prevailing mechanisms of fluid flow and transport depend on the porosity and permeability differences between fracture and matrix blocks (Huenges, 2010; Blöcher et al., 2010). If the rock matrix is more or less impermeable and has negligible porosity, the advective transport through the fracture will prevail. For the porous matrix block with negligible permeability, molecular diffusion will be the main transport mechanism rather than advection. If the porous matrix block and the fracture have permeabilities and/or transmissivities of the same order of magnitude, the transport of solutes (and heat) will take place simultaneously in the two media (fracture and matrix) by advection, dispersion and diffusion, depending on differences in pressure and concentration of solutes in the matrix blocks and fractures.

\section{Model setup, transport processes, and simulation parameters for tracer tests}

In order to explore (and quantify) the benefits of early-flowback sampling, and of multi-tracer use in conjunction with GPF and WF treatments, we consider two simplified fracture models based on lithostratigraphy and hydrogeology data from the Geothermal In-Situ Laboratory at Groß-Schönebeck in the NE-German basin. An 'EGS' of 'research project' character was developed at Groß-Schönebeck in crystalline and sedimentary formations, at a depth of 4147 to $4300 \mathrm{~m}$ (by WF treatment in a volcanic layer), and at 3968 to $4004 \mathrm{~m}$ (by GPF treatments in two sandstone layers), respectively (Blöcher et al., 2010; Zimmermann et al., 2010).

The commercially available finite-element software Feflow 5.4 (Diersch, 2009) was used to simulate fluid flow and tracer transport in fractures resulting from GPF and WF treatments, relying on some simplifying assumptions. The rock matrix is treated like a single-porosity medium in 3D, whereas vertical planar fractures are represented by lower-dimensional (2D) so-called 'discrete elements'. Flow and transport in fracture and matrix compartments are coupled to each other by Feflow's special, computationally efficient approach. Flow within 
WF-type fractures is assumed to obey the 'cubic law' (Tsang, 1992); flow within the matrix, as well as within GPF-type fractures, is assumed as Darcian. Thus, GPF-type fractures are treated almost like an ordinary porous medium, which (with the exception of solute exchange with the adjacent rock matrix) is similar to the 'push-pull' test situation considered by Schroth et al. (2001), with some limitations to be discussed in the next section. Solute transport in fractures, and within rock matrix is assumed as advective-dispersive, with a number of additional processes at fluid-solid interfaces as described below (adsorption-desorption onto/from matrix rock, or proppant coatings; matrix diffusion). The model has to be 3D because of its fracture (approximately radial) and matrix (approximately linear) flow and transport components. The assumption of horizontal and vertical symmetry and neglecting gravity effects means that we need to consider only one-quarter of the fracture plane, one-half of the fracture aperture or thickness and 1/8 of its adjacent matrix block (similar to the model used by Ghergut et al., $2013 \mathrm{~b}$ for parallel-fracture systems). The geometry of model compartments (fracture and matrix block) and the hydrogeological parameter distribution for the WF and GPF target layers are shown in Fig. 2.1.1. The assumption of isotropic permeability (described by a single scalar value for each compartment) is rather unrealistic, but it does not influence the parameter sensitivity behavior of simulated tracer signals. Further, we assume isothermal conditions; the hydraulic conductivity values listed in Fig. 2.1.1 corresponds to a temperature of $120^{\circ} \mathrm{C}$, assumed to prevail during tracer injection and early flowback, as a transient 'equilibrium' between native reservoir conditions $\left(\sim 160^{\circ} \mathrm{C}\right)$ and injectate temperature $\left(\sim 15^{\circ} \mathrm{C}\right)$. 


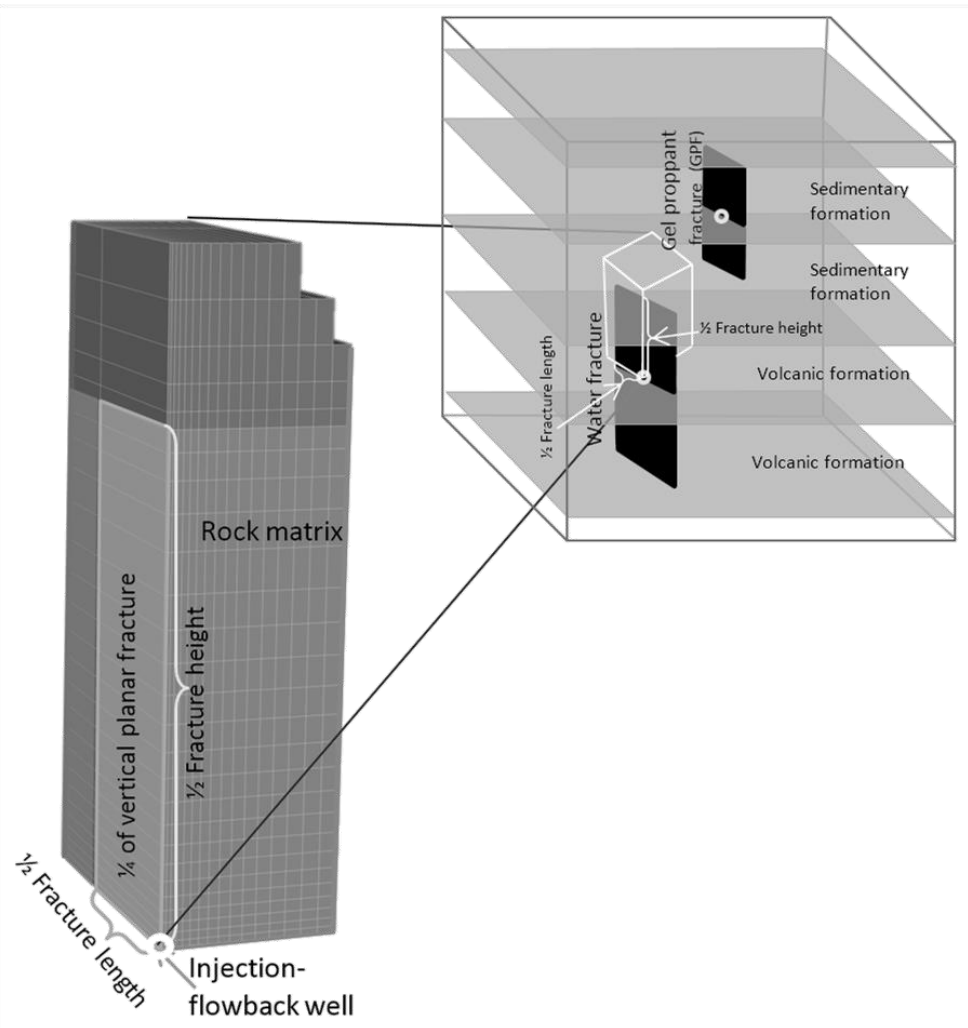

Figure 2.3.1: 3D model domain and hydrogeological parameter distribution (after Blöcher et al., 2010)

Table 1: Values of matrix and fracture parameters in WF and GPF model

\begin{tabular}{l|l|l|l}
\hline \multicolumn{2}{l|}{} & Water fracture (WF) & $\begin{array}{l}\text { Gel proppant fracture } \\
\text { (GPF) }\end{array}$ \\
\hline $\begin{array}{l}\text { Hydraulic } \\
\text { conductivity }\end{array}$ & $\begin{array}{l}\text { Stimulated layer } \\
\text { Fracture arrest } \\
\text { layer }\end{array}$ & $\begin{array}{l}3.2 \times 10-9 \mathrm{~m} / \mathrm{s} \\
3.2 \times 10-9 \mathrm{~m} / \mathrm{s}\end{array}$ & $\begin{array}{l}6.4 \times 10-8 \mathrm{~m} / \mathrm{s} \\
1.6 \times 10-9 \mathrm{~m} / \mathrm{s}\end{array}$ \\
\hline $\begin{array}{l}\text { Porosity } \\
\text { Stimulated layer } \\
\text { Fracture arrest }\end{array}$ & $\begin{array}{l}0.1 \% \\
0.5 \%\end{array}$ & $3 \%$ \\
$\begin{array}{l}1 / 2 \text { Fracture height } \\
\text { layer }\end{array}$ & $100 \mathrm{~m}$ & $1 \%$ \\
$\begin{array}{l}\text { Fracture porosity } \\
\text { Fracture aperture (WF)/thickness } \\
(\text { GPF }\end{array}$ & $0.18 \mathrm{~mm}$ to $1.08 \mathrm{~mm}$ & $60 \mathrm{~m}$ \\
\hline
\end{tabular}

Fracture apertures were varied between $0.2 \mathrm{~mm}$ and $24 \mathrm{~mm}$, the lower of these values being regarded as more representative for fractures resulting from WF treatments, and the higher values for fractures resulting from GPF treatments (Table 1). Within GPF-type fractures, 
proppant-packing porosity was varied between $30 \%$ and $60 \%$. Longitudinal dispersivities within fractures were varied in the range of few meters, their effect on simulated tracer signals was considered (see next section). Transverse dispersivities were assumed to be twenty times lower. Dispersivity values for the rock matrix, likely in the range of centimeters-decimeters, did not show any notable influence on simulated tracer signals, as advective penetration of the matrix remained negligible. In the sequel, whenever the term 'dispersivity' is used, it pertains to longitudinal dispersivity within fractures. In order to avoid confusion between 'total aperture' and 'effective aperture' (equal to the product of 'total aperture' and intra-fracture porosity), we prefer to reserve the term 'aperture' for the WF type (with $100 \%$ porosity), and denote the total aperture of a GPF-type fracture as 'thickness'. The notion of aperture is context-dependent; the systematic difference, both in theory and in field measurements, between 'hydraulic aperture', 'tracer aperture', and 'tracer-hydraulic aperture' was explained by Tsang (1992) as the difference between 'cubic law aperture', 'mass balance aperture', and 'frictional loss aperture'; aperture measurements should always be regarded as context dependent (Berkowitz, 2002). In the sense of Tsang (1992), the findings in the present paper pertain to 'mass balance aperture' only.

For tracer tests conducted in conjunction with WF and GPF treatments, we consider three classes of tracers: conservative (in particular, non-sorbing) tracers, matrix-sorptive tracers (MST), and proppant-sorptive tracers (PST). In any kind of test, whether with WF or GPF treatments, at least one conservative tracer shall always be injected; within the conservativetracer class, diffusion coefficients were varied in order to probe the usefulness of matrix diffusion processes for parameter determination from early flowback signals (the result being largely negative). For obvious reasons, PST can be used only with GPF treatments, whereas MST can in principle be used both with WF and with GPF treatments. The idea that PST and MST can -and ought to be assumed different from each other (distinct groups of chemicals) is justified by the chemical specificity of adsorption processes: roughly speaking, "different species have a different adsorption affinity to different solid surfaces". Not only should MST differ from PST, MST for volcanics will likely be different from MST for sandstone or claystone. Proppant particles can be prepared with specific coatings to ensure sorptivity for certain tracers.

Given the relatively low flow velocities, the processes of tracer adsorption-desorption (onto/from rock matrix or proppants) can be assumed to be at equilibrium, and given the typically low tracer concentrations, sorption distribution laws can be assumed as linear (Freeze 
and Cherry, 1979), which allows the effects of sorption-desorption on tracer transport to be described simply by a dimensionless retardation factor (Bouwer, 1991; Kinzelbach and Rausch, 1995; Schroth et al., 2001), defined as:

$R=1+\rho \times K_{d} \times \frac{1-n}{n}=1+\kappa \times \frac{1-\mathrm{n}}{\mathrm{n}}$

where:

$n=$ porosity of the flow medium (either rock matrix, for MST, or proppant packing, for PST)

$\rho=$ intrinsic density of solid-phase material (either rock, or proppants)

$K_{d}=$ sorption distribution coefficient (slope of linear segment of adsorption isotherm, Freeze and Cherry, 1979)

$\kappa=\rho \times K_{d}=$ dimensionless sorption distribution coefficient

The retardation factor $R$ is not an intrinsic tracer property, but it also depends on the porosity of the transport medium; thus, strictly speaking, $R$-values cannot be varied independently of porosity. Laboratory tests on tracer sorptivity, conducted prior to tracer use in field tests, measure $K_{d}$ or $\kappa$, not $R$ values. However, some of the simulation results presented in the next sections are better described in terms of $R$.

\section{Spatial discretization and hydraulic treatment of injection-flowback tests}

The horizontal extent of the model domain was chosen to be at least twice the maximum tracer travel distance. The fracture and matrix block are shown in Fig. 2.1.1 was discretized by up to 58280 elements. A stronger retardation by adsorption/desorption implies a shorter travel distance for that particular tracer species. This implies that spatial discretization (and hence numerical dispersion) may vary from tracer to tracer. The numerical dispersion was investigated in a separate study (Karmakar, in prep., Chapter 2, part 3).

The timing of tracer pulse and fluid (chaser) injection and flowback for both WF and GPF treatments are presented in Fig. 2.1.2. The total simulation time of up to 2 days were discretized by up to 2100 time steps of variable duration, resulting from time-step control by predictorcorrector schemes implemented in FEFLOW. The initial time step was set to $10^{-9}$ day, and the time step increase factor was limited to 1.2 , as injection duration was very small, and the shift from injection to pumping was assumed to take place over a very short time interval. 


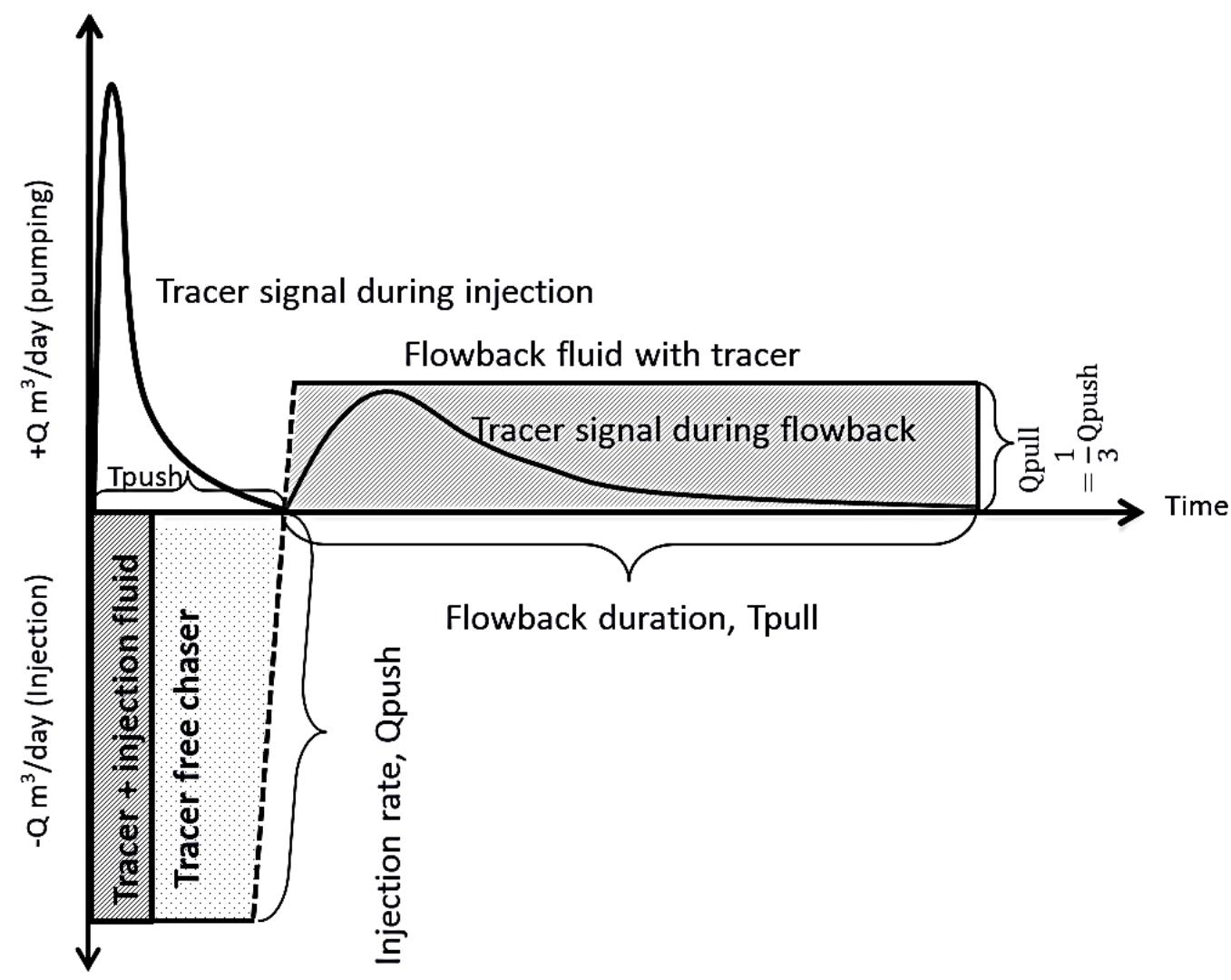

Figure 2.4.1: Fluid and tracer injection-flowback in GPF and WF treatments. The transition from constant injection to constant flowback was approximated as linear (dashed-line segment); its duration is enlarged for better recognition. In the simulations, the transition spans only 1/60 of Tpush.

Injection rate and duration were set as similar for all tracer species corresponding to a chaser injection volume of about half the typical fracture. This is a reasonable compromise between the requirement that 'most tracers stay within the fracture' and the fact that fracture aperture is not known prior to the test. The chaser fluid (water) was injected in pulses to move the tracer to a certain distance inside the fracture. An advantage of this particular SW approach is that it does not rely on substantial advective penetration into the rock matrix. The flowback volume (Fig. 2.1.2) can be significantly larger than the tracer chaser volume injected immediately prior to it. The flowback results from pressure buildup by the entire treatment process, with total injectate volumes being much larger than the tracer-chaser volume (especially for WF treatments). We recall that tracer and chaser are added only at the end of the treatment. In fact, flowback rate and duration are independent of the tracer-chaser injection rate and duration. Without external control, flowback would start at high rates and then decline more or less exponentially, but in practice often some control is applied to maintain it at an approximately constant rate; thus, for simplicity, the scheme is shown in Fig. 2.1.2 was used. 


\section{Parameter interplay, and numerical simulations}

The study by Schroth et al. (2001) on the in-situ evaluation of solute retardation using SW push-pull indicates that in a homogeneous single-porosity medium tracer 'pull' signals depend on porosity, thickness, and longitudinal dispersivity through the following product

(porosity $\times$ thickness $) \times($ dispersivity squared $)$, or

(effective aperture) $\times($ dispersivity squared $)$

It, therefore, follows that these three parameters cannot be determined independently from each other. Dispersivity has a stronger influence than porosity or thickness - a fact that can become useful as will be seen in the next section. In groundwater remediation application considered by Schroth et al. (2001), this hydrogeological parameter interplay was not an issue, since the aquifer thickness and porosity were known in advance of conducting the SW tracer push-pull tests, and the unknown parameters were aquifer dispersivity and solute retardation. By contrast, in the present study, all these parameters are unknown (three parameters for GPF: thickness, porosity, dispersivity; two parameters for WF: aperture, dispersivity); however, the tracerproppant/rock sorptivity behavior is assumed as known in advance.

The closed-form approximation to tracer 'pull' signals used by Schroth et al. (2001) may not be applicable to our WF and GPF tracer test scenarios. In fact, formulae (9) - (11) used by Schroth et al. (2001, pp. 107-108) are valid only in the low-dispersivity limit, a requirement very likely violated by fractures resulting from GPF or WF treatments in natural geothermal reservoirs. Consequences of parameter interplay on the parameter estimation from measured tracer signals in natural systems need not be as dramatic as suggested by product (2). As an example, the product formula implies that a tenfold increase in dispersivity will produce the same effect on tracer signals as a 100-fold increase in porosity, thickness, or effective aperture. However, with porosity expected to be between $30 \%$ and $60 \%$, and thickness or aperture ranging from $\sim 1 \mathrm{~mm}$ to $\sim 1 \mathrm{~cm}$, a 100x variation cannot actually occur. Thus the fact that dispersivity has a stronger effect on SW tracer signals than the other parameters, in conjunction with relatively narrow ranges for parameters like proppant-packing porosity, GPF thickness or WF aperture, may allow to at least roughly estimate dispersivity without knowing the exact values of other parameters. A possible workaround to parameter interplay is the use of semi-empirical correlations between hydrogeological parameters to constrain the value of either dispersivity as a function of fracture aperture and porosity (Horne and Rodriguez, 1981; Fossum and Horne, 1982; Pruess and Bodvarsson, 1984), or porosity as a function of permeability (Guimerà and 
Carrera, 1997), or proppant-packing dispersivity as a function of proppant treatment protocols (Tomac and Gutierrez, 2015).

Alternatively, it may be possible to make priori assumptions on the magnitude of the Peclet number $(\mathrm{Pe})$ of the overall transport scenario (with Pe defined as the ratio between tracerinvaded fracture radius and longitudinal dispersivity). For the vast majority of transport scenarios in fractured-porous media of practical relevance, Peclet numbers range between 8 and 20. Dispersion within fractures can result from multiple processes - the most significant of which include: 'Taylor-Aris dispersion', 'roughness dispersion', 'aperture-variation dispersion' (Bauget and Fourar, 2007); it is not possible to individually quantify these processes from early-flowback observations. There is an important difference between the SW tracer test scenario of Schroth et al. (2001) and the GPF and WF tracer test scenarios considered in the present paper. Whereas the transport of conservative and PST in GPF-type fractures is approximately similar to tracer transport in the 'aquifer cylinder' of Schroth et al. (2001), the transport of

(i) 'matrix-diffusive' tracers in thin WF-type fractures and

(ii) MST in any fracture type

does not fit into the model considered by Schroth et al (2001); the latter model excludes tracer exchange across 'cylinder' top and bottom (corresponding to fracture walls, or the fracturematrix interface). Owing to tracer flux across fracture walls, WF aperture and GPF thickness are expected to influence MST and possibly 'matrix-diffusive' signals in addition to the product parameter (2). As to matrix diffusion, one should not expect it to have a notable effect in shortterm tests in GPF-type fractures, but it might have some impact in thin WF-type fractures.

Various studies (Kocabas and Horne, 1987; Pruess and Doughty, 2010; Jung and Pruess, 2011; Maier and Kocabas, 2013) indicate that thin-fracture apertures can be estimated from SW pushpull tests using conservative tracers or heat exchange, relying on matrix diffusion; however, considerably longer observation times are required than those considered in this early-flowback study. The process of MST exchange at fracture walls largely resembles that of matrix diffusion, but unlike matrix diffusion, equilibrium is attained within a very short time. It is, therefore, reasonable to expect that MST may be helpful for estimating WF aperture and GPF thickness independently of other hydrogeological parameters. 
Based on above considerations, the numerical simulations of tracer tests in fractures resulting from GPF and WF treatments were designed to:

1.) probe the effects of dispersivity, and its interplay with porosity and thickness or aperture, on conservative tracer signals in GPF and WF (for obtaining a rough estimate of dispersivity);

2.) explore the effects of porosity and thickness or aperture in MST in GPF and WF (for determining GPF thickness and WF aperture);

3.) explore the effects of porosity and thickness on PST in GPF (for determining proppantpacking porosity in GPF).

\section{Results}

In this section, we present numerical simulation results focusing on the following four issues: (i) how does each parameter (dispersivity, WF aperture, GPF thickness and porosity) influence the 'peak arrival time', and the early amplitude of simulated tracer signals? Signal tails are ignored, since parameter determination is based only on early flowback; (ii) is the influence monotonic with respect to a parameter over its relevant range of values? (iii) if parameter interplay occurs, to what extent does it impede parameter determination? (iv) do the signals of sorptive tracers show less interplay and/or improved sensitivity to relevant parameters, compared to conservative-tracer signals?

The slight 'bump' at the beginning of flowback signals, seen in some of the simulated tracer signals, is due to the representation of injection-flowback hydraulics. The rates of injection and flowback were set as constant, with a short linear transition interval between them (indicated by dashed-line segments on Fig. 2.1-2); instead of letting the variable flowback rate naturally adjust to the actual outflow boundary condition. This 'bump' does not affect parameter sensitivity or estimation since it is not associated with any special 'arrival time' that may be used for parameter determination.

\subsection{Conservative-tracer signals during early flowback}

Figs. 2.1- 3 and 2.1- 4 demonstrate the influence of dispersivity $\left(\alpha_{\mathrm{L}}\right)$ on conservative-tracer signals during early flowback in GPF-type fractures and its interplay with fracture thickness and proppant-packing porosity. In Fig. 2.1.3, porosity is kept fixed while thickness is varied whereas in fig. 4 the thickness is kept fixed while porosity is varied. As expected from the discussion in a previous section, the product-type parameter interplay (2) is seen to occur, but 
the influence of dispersivity is significantly stronger than that of thickness or porosity. Thus, dispersivity can be roughly estimated, though additional / a priori knowledge from other sources would be helpful. Signal amplitude decreases monotonically with increasing dispersion, whereas dispersion influence on 'peak arrival time' is insufficient for parameter estimation purposes.

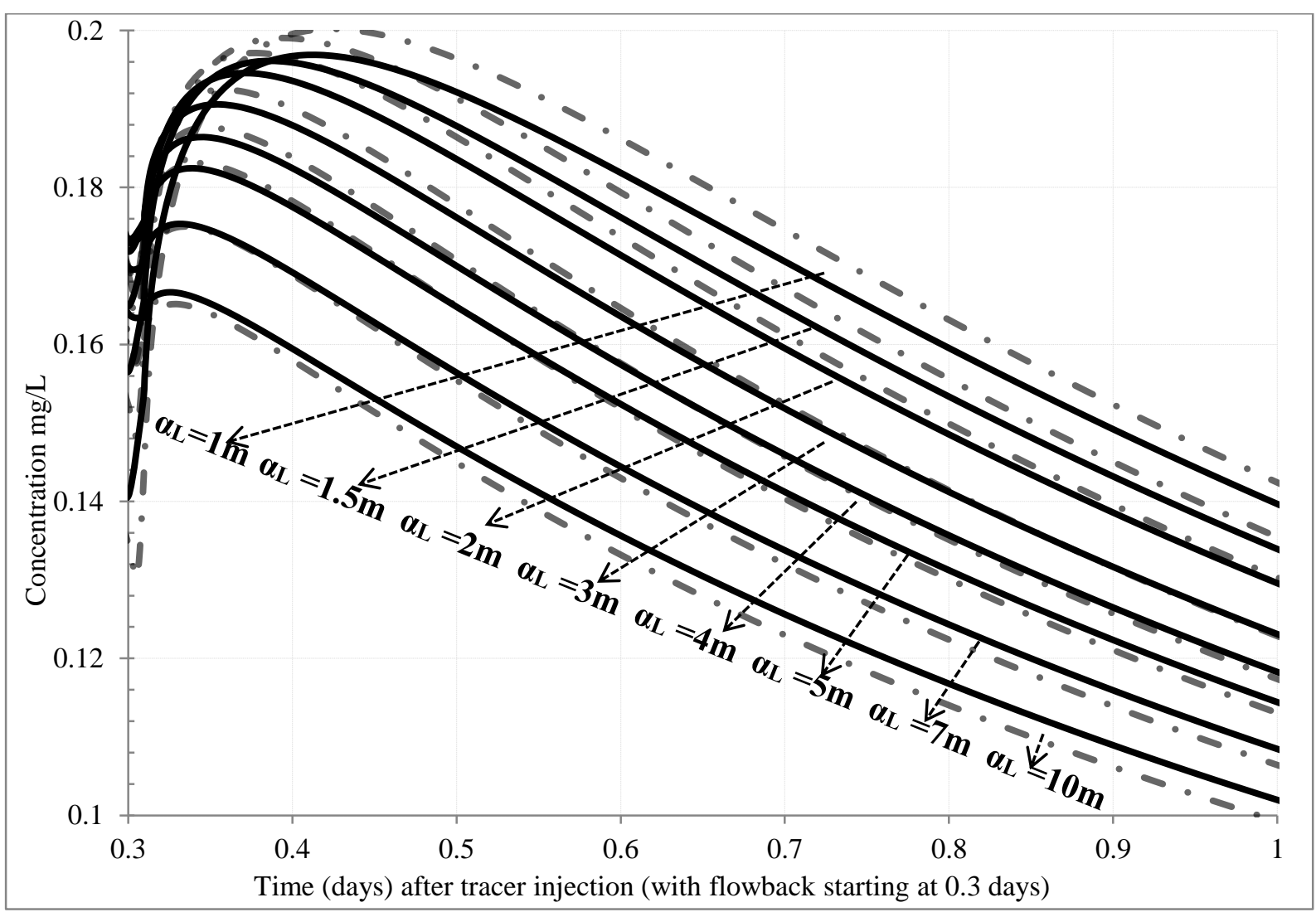

Figure 2.6.1: Simulated signals of conservative tracers during GPF flowback: the effect of dispersion processes (expressed by eight different values of longitudinal dispersivity $\alpha L$, as indicated by signal labels, in meters), shown for two different values of GPF thickness (solid line: $12 \mathrm{~mm}$, dashed-dotted line: $20 \mathrm{~mm}$ ), with a fixed value of GPF porosity (45\%). 


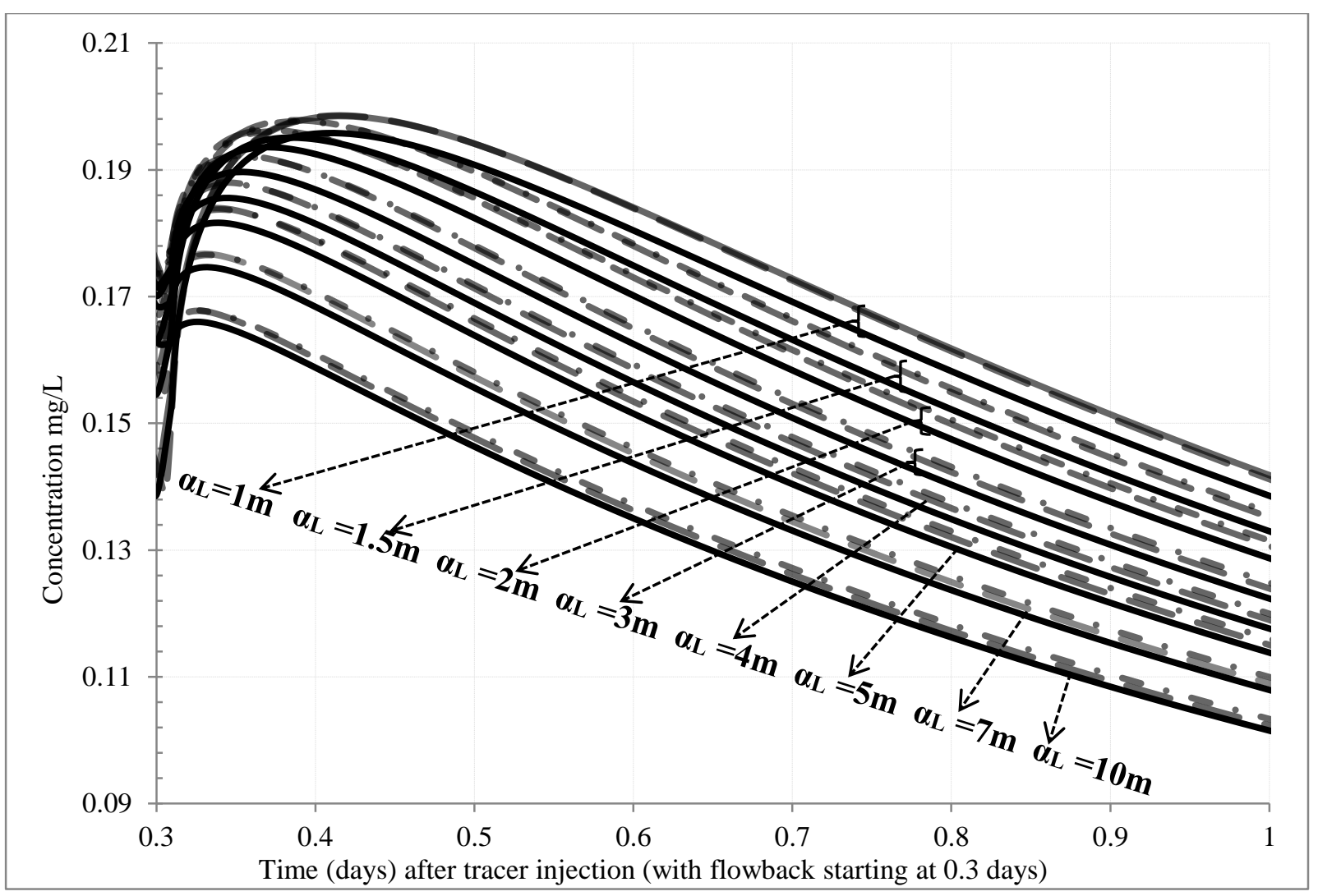

Figure 2.6.2: Simulated signals of conservative tracers during GPF flowback: the effect of dispersion processes (expressed by eight different values of longitudinal dispersivity $\alpha L$, as indicated by signal labels, in meters), shown for three different values of GPF porosity (solid line: 30\%, dashed line: 45\%, dashed-dotted line: 60\%), with a fixed value of GPF thickness (12mm).

Fig. 2.1.4 demonstrates the influence of dispersion and fracture aperture on conservative-tracer signals during early flowback in WF-type fractures. Unlike in the GPF case, there is a significant monotonic increase of 'peak arrival time' with decreasing dispersivity, whereas aperture is seen to have no significant influence on 'peak arrival time'. There is the interplay between dispersivity and aperture with regard to signal amplitude, but the dispersivity value identified based on 'peak arrival time' can be used to determine aperture. Unlike in the GPF case, signal slopes vary strongly, both in time and in parameter space. 


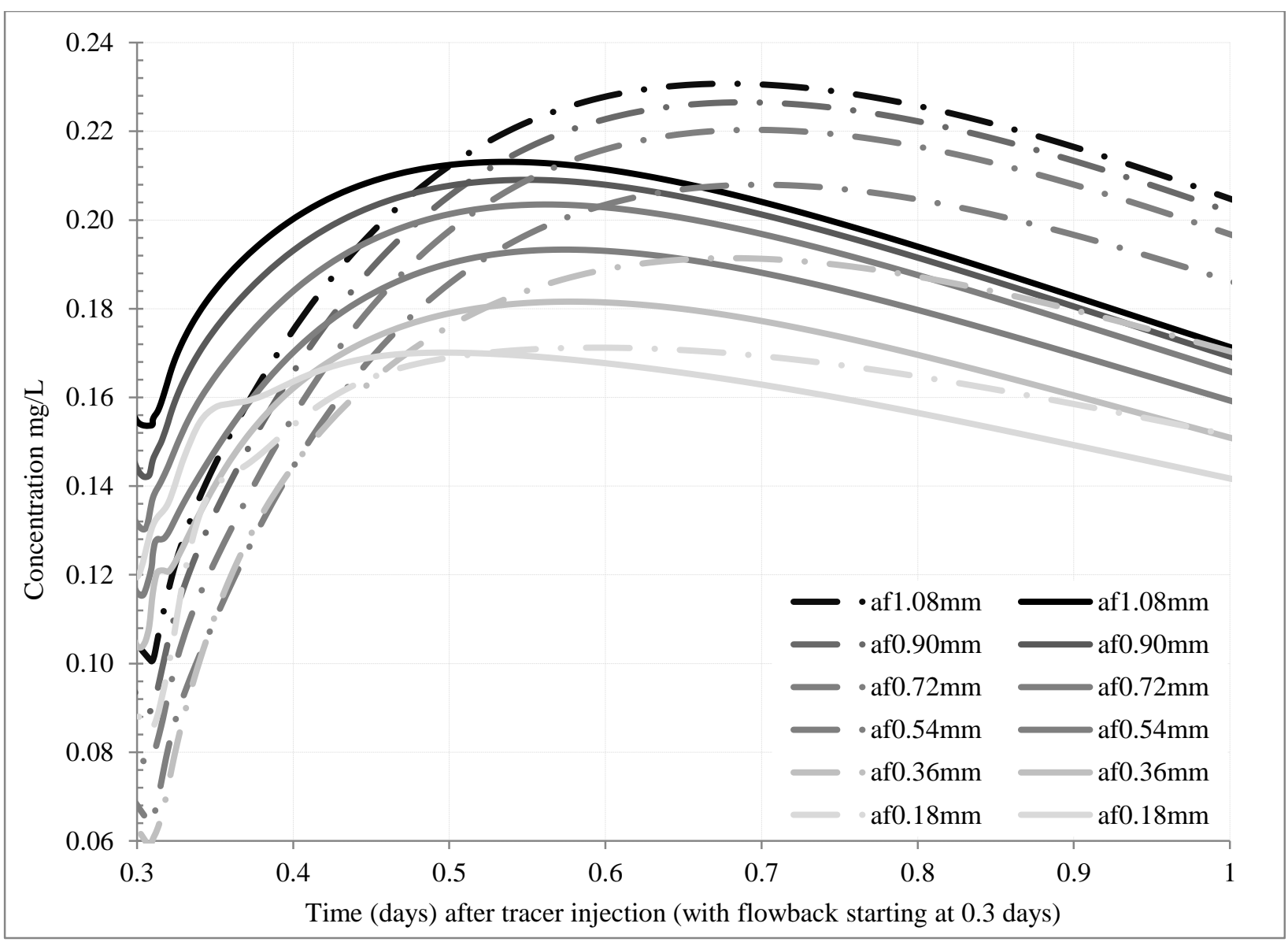

Figure 2.6.3: Simulated signals of conservative tracers during WF flowback: the distinct effects of fracture aperture (with values represented by different shadings as shown by legend) and of longitudinal dispersivity (solid line: $7 m$, dash-dotted line: $5 m$ ).

\subsection{MST signals during early flowback}

In Figs. 2.1.8-10, the transport of MST in GPF-type fractures is explored from two different angles: (1) comparison between MST and matrix diffusion processes; and (2) parameter determination. Fig. 6 (produced with fixed values of GPF thickness and porosity) demonstrates that increasing MST sorptivity lowers the tracer signal peak amplitude (approximately inversely proportional to $R^{1 / 2}$ during early flowback), without influencing the apparent 'peak arrival time'. This is somewhat analogous to the effects of increasing diffusion coefficients in the context of matrix diffusion in SW push-pull tests (cf. Ghergut et al. 2013b). Fig. 2.1.9 illustrates that the sensitivity to fracture thickness improves with increasing sorptivity; this was confirmed over the entire parameter range of interest. The thickness sensitivity of any MST is greater than that of a non-sorptive tracer, and increases with decreasing thickness; on the other hand, signal amplitude increases monotonically with increasing thickness, and decreases with 
increasing sorptivity. Fig. 2.1.8 demonstrates that the MST signals are independent of proppant-packing porosity; and the GPF thickness can be determined independently of porosity.

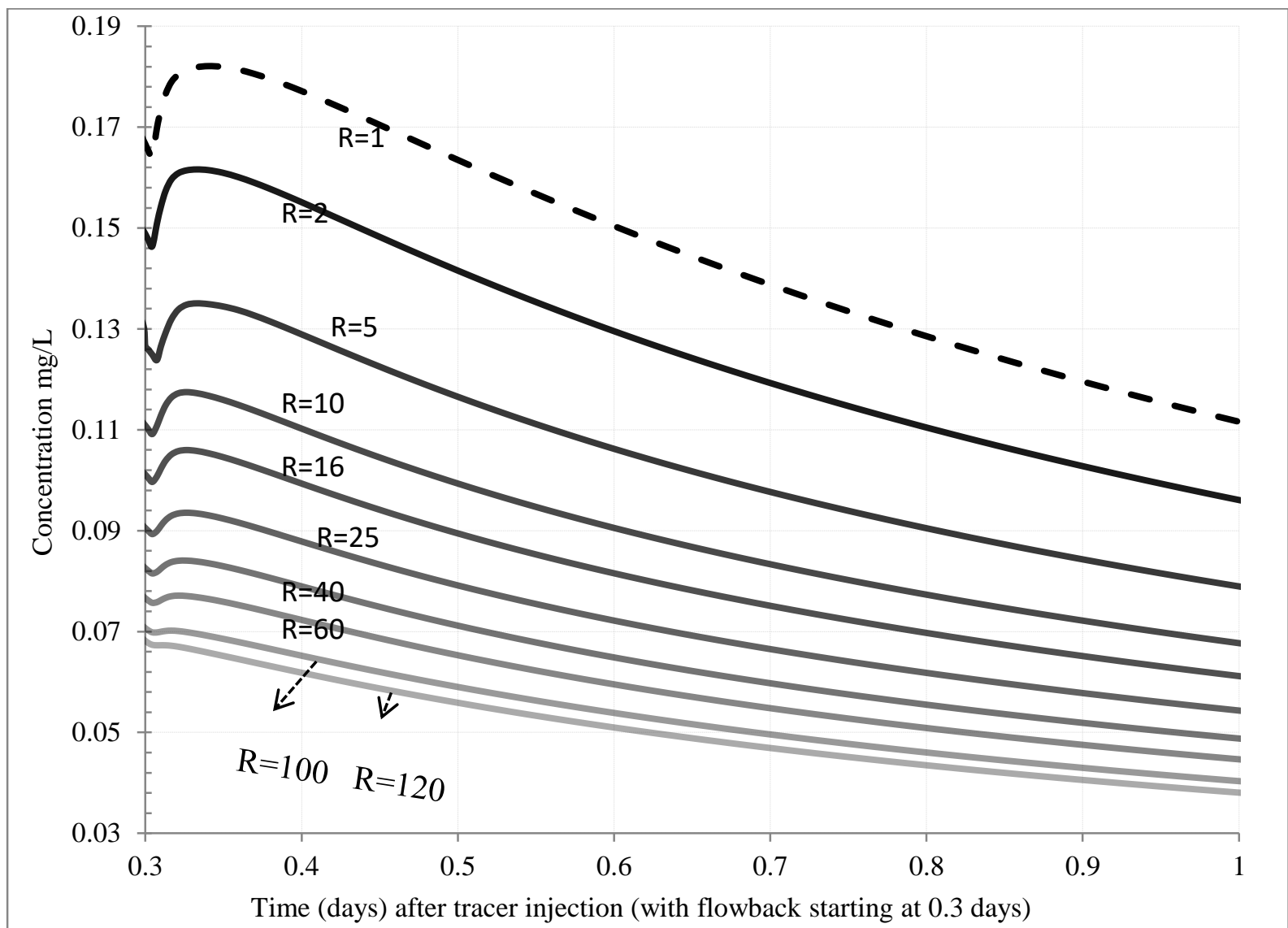

Figure 2.6.4: Simulated signals of various MST (characterized by different Kd values) during GPF flowback, at a fixed value of GPF thickness (12 $\mathrm{mm}$ ) and proppant-packing porosity 30\%). Signals are labeled by the retardation factor $R$, instead of $K d$ values the signal of a conservative (non-sorptive, $K d=0, R=1$ ) tracer is shown for comparison. 


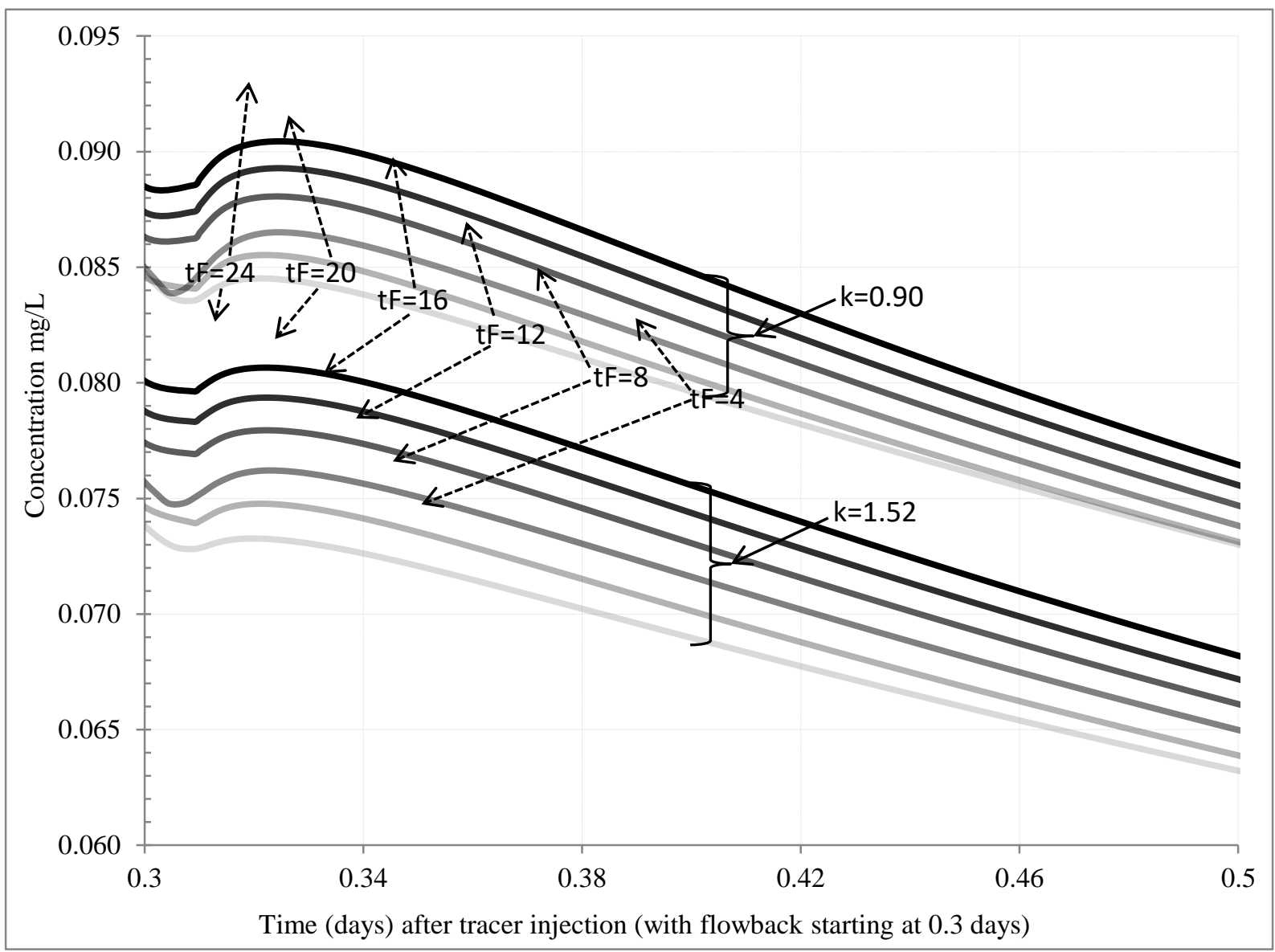

Figure 2.6.5: Simulated signals of two MST during GPF flowback: the effect of GPF thickness (values are indicated as signal labels, in $\mathrm{mm}$ ), with a fixed value of proppant-packing porosity (45\%), shown for two tracer species, a less sorptive one (dimensionless $\kappa=0.9$ ), and a more sorptive one (dimensionless $\kappa=1.52$ ). 


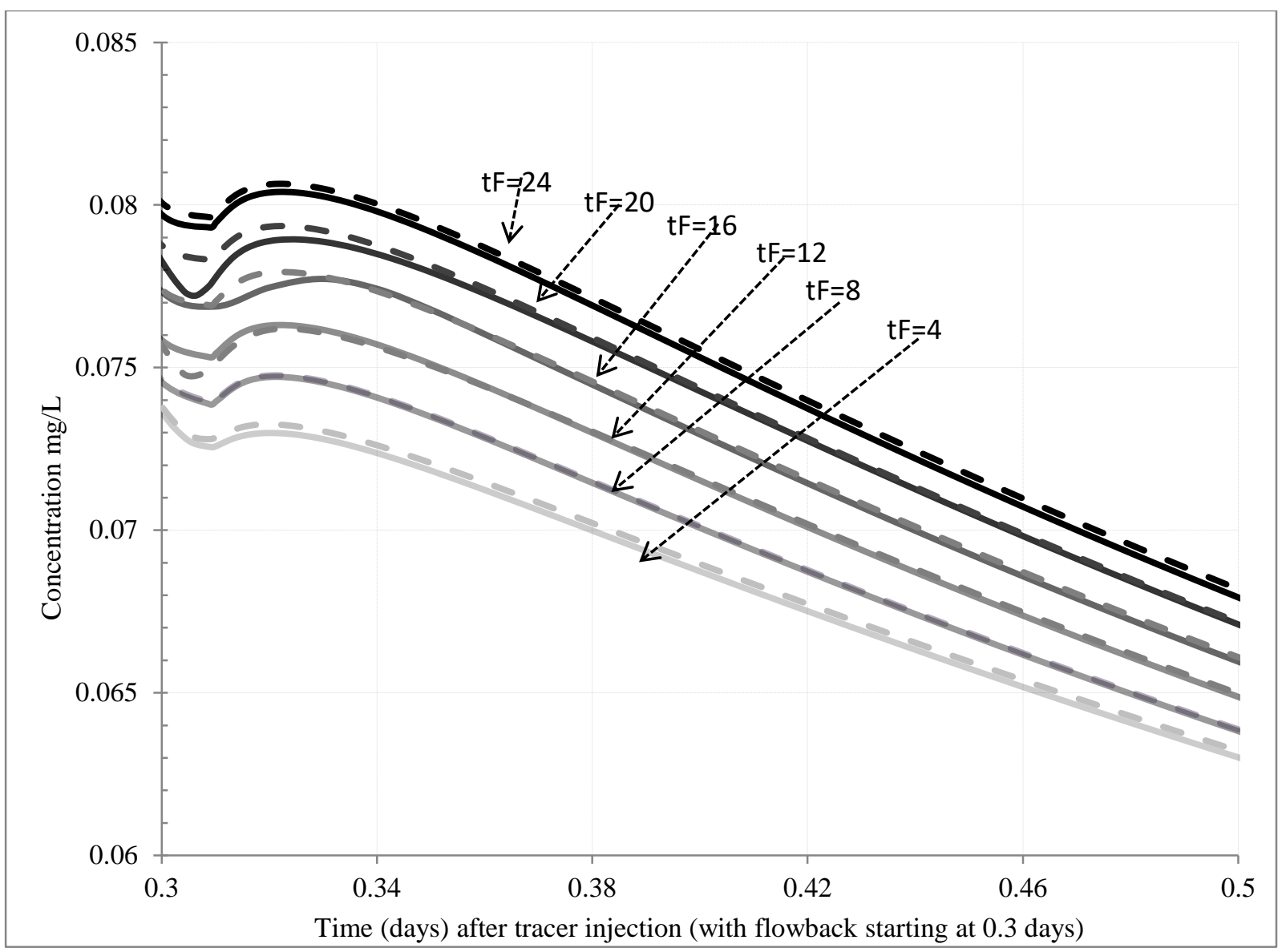

Figure 2.6.6: Simulated signals for one MST (characterized by dimensionless $k=1.5$ ) during GPF flowback: the effect of GPF thickness (values are indicated as signal labels, in $\mathrm{mm}$ ), with two values of GPF porosity (solid line: 30\%, broken line: 60\%)

For MST signals in WF-type fractures (not shown here), a largely similar behavior was found. Sensitivity to aperture is improved, compared to that for non-sorptive tracers, and sensitivity increases with decreasing aperture. On the other hand, signal amplitude increases monotonically with increasing thickness and decreases with increasing sorptivity. Deviations from the above-described behavior (and a slight influence on apparent 'arrival times') are seen to occur for values outside the plausible range for GPF thickness or WF aperture.

\subsection{PST signals during early flowback in GPF fractures}

Finally, the transport of PST in GPF-type fractures is explored from two different angles i.e., parameter interplay, and porosity determination. Fig. 2.1.11 illustrates the interplay between GPF-type fracture thickness and proppant-packing porosity. Signal amplitude decreases with increasing fracture thickness but increases with increasing porosity. 
Fig. 2.1.10 demonstrates that once the value of GPF-type fracture thickness was identified (using an MST tracer as described in section 6.2), the proppant-packing porosity can be determined, provided fracture thickness is about $\sim 1 \mathrm{~cm}$ or higher. If GPF-type fracture thickness is less than $0.5 \mathrm{~cm}$ (which is implausible), PST signal sensitivity to porosity becomes too low.

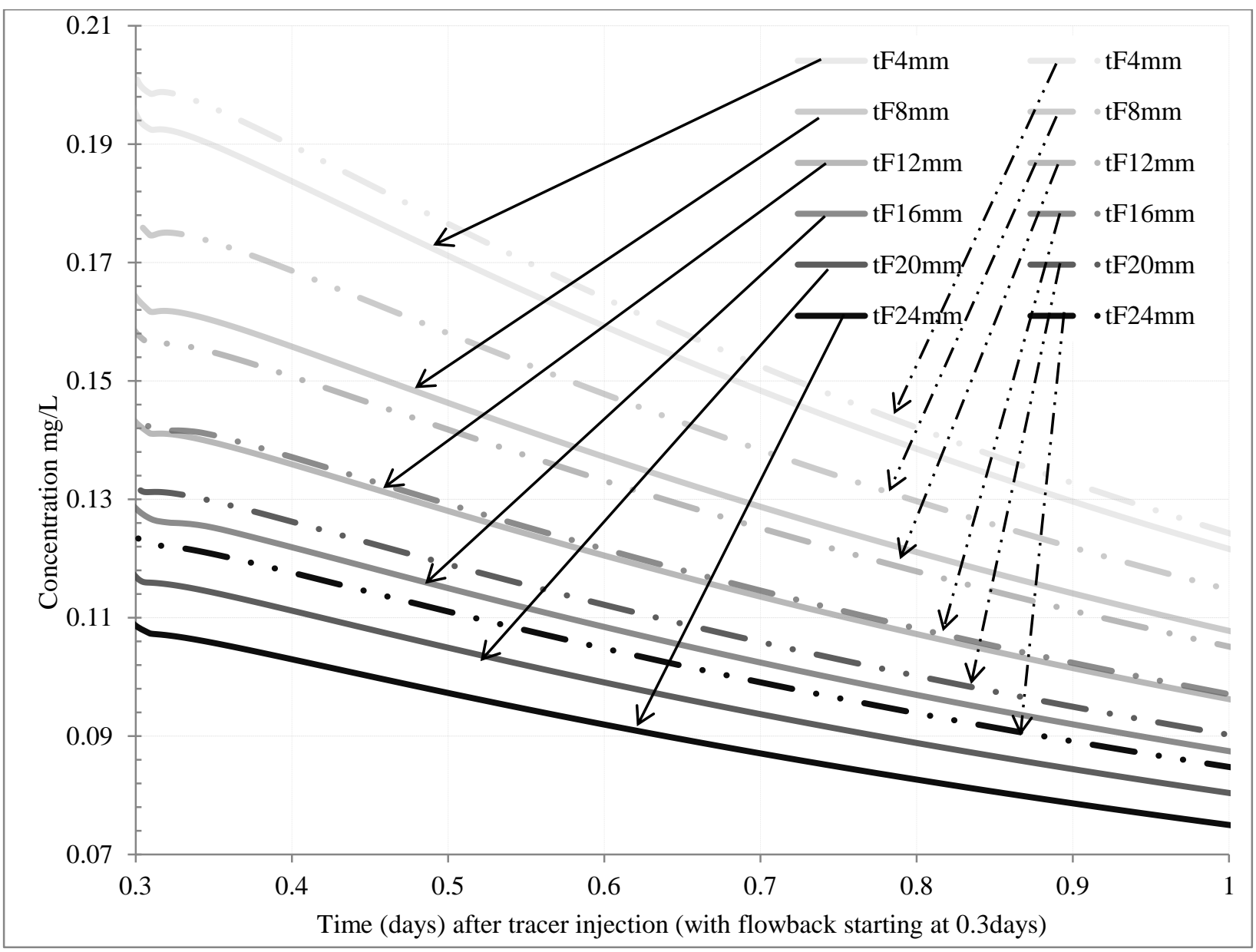

Figure 2.6.7: Simulated signals for one PST (characterized by dimensionless $k=40$ ) during GPF flowback: the effects of GPF thickness and of GPF porosity (solid lines: 35\%, broken lines: 55\%) illustrate parameter interplay. 




Figure 2.6.8: Simulated signals for one PST (characterized by dimensionless $k=40$ ) during GPF flowback: the effect of GPF thickness (values are indicated in $\mathrm{mm}$ ), and the effect of GPF porosity (gray tones, from light: 30\%, to dark: 60\%), illustrating the increase of porosity sensitivity with increasing thickness.

\section{Discussion, and recommendations for future tracer tests}

Owing to flow-field reversal by the SW test configuration, two 'unusual' behaviors occur, opposite to those from inter-well tracer tests: 'retardation' factors are not directly reflected by the apparent 'arrival times' (cf. discussion in Ghergut et al., 2013a), whereas dispersion determines the apparent 'arrival time'. Moreover, this latter effect is strong enough for conservative tracers in thin WF-type fractures to enable dispersivity and aperture determination independently of each other. For thicker, GPF-type fractures, a stronger parameter interplay is found between dispersivity, fracture thickness and proppant-packing porosity. However, a rough estimation of dispersivity from conservative tracer signals is still possible since dispersivity effects are stronger than those of GPF thickness and proppant-packing porosity. Once dispersivity is identified (by this or some different approach as suggested in section 5), 
GPF-type fracture thickness and proppant-packing porosity can be determined independently of each other, if suitable MST and PST data are available. The use of an MST enables thickness determination; and a PST enables proppant-packing porosity determination if the thickness is not too small. For WF-type fractures, aperture determination is possible with conservative tracers alone, but the use of MST improves sensitivity. Parameter determination for GPF-type fractures only becomes possible with MST and PST.

Regarding the use of conservative tracer pairs with contrasting diffusivity, simulations have shown that matrix diffusion effects are far too weak to enable parameter inversion from early flowback signals. Alternatively, heat could be used as a 'matrix-diffusive tracer' instead of solute tracers, owing to heat diffusivities being much higher than solute diffusivities (Kocabas and Horne, 1987; Pruess and Doughty 2010; Jung and Pruess, 2011; Maier and Kocabas, 2013). However, during early flowback following multiple injectate slugs with different temperatures, it may be difficult to use "heat" for determining fracture parameters.

Based on Figs. 2.1.3-5, 2.1.8 and 2.1.10 one can make some recommendations regarding the timing and frequency of tracer signal sampling. For WF-type fractures, dense sampling at the beginning of flowback (at least 30 samplings during the very first day, i.e. before recovering the first $1 / 10$ of the injected chaser volume) is necessary, if both dispersivity and aperture are to be determined. By contrast, for GPF-type fractures, sparse uniform sampling ( 10 samplings uniformly distributed over a flowback volume of the same order as the chaser volume) should suffice for fracture parameter estimation - if suitable MST and PST are available. For WF-type fractures essential tracer information can be contained in the very first hours of flowback and for GPF-type fractures, the flowback corresponding to a recovery between $1 / 3$ and $2 / 3$ of chaser volume is likely to yield the most useful information.

To identify the minimum sorptivity (minimum $\kappa$ value) required to induce sufficient contrast between measurable tracer signals for different values of target parameters, the scheme to follow is: a $p \%$ change in a target-parameter value produces $c \%$ change in the signal of a conservative tracer, and a $s \%$ change in the signal of a sorptive tracer, $s$ being a function of $\kappa$. One then needs to find out the minimum value of $\kappa$ that renders $s$ significantly higher than $c$. This value cannot be prescribed in a universal manner because the very meaning of 'significantly higher' depends on how tracer signals can be sampled and measured, a process always affected by various errors. In any case, the higher the value of $\kappa$, the higher is the sensitivity improvement. On the other hand, the higher the tracer's retardation, the lower is its 
fracture invasion, and consequently a poorer capability for characterizing the fracture as a whole. In principle, this could be compensated by increasing the chaser volume (i.e., by injecting sorptive tracers earlier than conservative tracers). The effect of different injection durations (different chaser volumes) on tracer signals was investigated in a parallel study (Karmakar et al., 2015).

Fracture thicknesses based on neutron ray detection and the use of proppants with activated coatings (McDaniel et al., 2009) can provide enough data to determine fracture opening during the EGS development and monitoring phases. However, the neutron rays commonly detected by gamma probes may be affected by the presence of neutron-ray absorbing or emitting substance in the rock mass or by barriers across the fracture opening that impair detection. In the latter case, a tracer-based SW test would be advantageous for obtaining reliable data. The early tracer signal can be employed to characterize fracture thickness and porosity by using two tracers that are only retarded by either the proppant or the matrix. Variable injection rates were found to be effective during WF stimulation rather than a constant flow rate ("cyclic stimulation", Zimmermann et al, 2010). The tracer-based procedure described in the present paper is also applicable in such cases; tracers should be added shortly before the end of the last injection cycle.

In summary, this simulation study has produced two significant results: (1) water fracture aperture can be effectively evaluated based on early-time tracer signals of a conservative tracer; and (2) by using the combination of matrix sorptive and proppant sorptive tracers, it is possible to estimate fracture thickness and porosity in gel proppant fractures from a single test.

The injection and flowback of a small fluid volume, and thus little dilution of the injected tracers, has three practical advantages: (1) there is no need to inject large tracer quantities; (2) one does not have to wait for the tails of the test signals; and (3) the field and laboratory monitoring of the tracer signals does not have to be conducted for ultra-low tracer concentrations, which is known to be a major challenge, with the highly-mineralized and especially high organic content fluids typically encountered in the NE German Basin at 3-5 km depth.

Acknowledgments: The first author gratefully acknowledges continued financial support from the DAAD (German Academic Exchange Service) to pursue his Ph.D. work. This work also benefited from long-term financial and technical support granted by the Federal German 
Ministry for Economic Affairs and Energy (BMWi) within the project TRENDS (2014-2018, FKZ 0325515), as well as by Baker Hughes (Celle) and the Lower Saxonian Science and Culture Ministry (MWK Niedersachsen) within the interdisciplinary research project "gebo Geothermal Energy and High-Performance Drilling” (2009-2014). Last, not least, the authors gratefully acknowledge the reviewers Dr. Marcelo Lippmann, Mr. Michael G. Shook, as well as the Editors for their valuable help in improving this manuscript.

\section{References}

Bauget, F., Fourar, M., 2007. Convective Dispersion in a Real Fracture. In: Proceedings $32^{\text {nd }}$ Workshop on Geothermal Reservoir Engineering, Jan. 22-24, 2007, Stanford Univ. (CA), SGP-TR-183.

Berkowitz, B., 2002. Characterizing flow and transport in fractured geological media: A review. Adv. in Water Resour. 25, 861-884.

Blöcher, G., Zimmermann, G., Moeck, I., Brandt, W., Hassanzadegan, A., Magri, F., 2010. 3D numerical modeling of hydrothermal processes during the lifetime of a deep geothermal reservoir. Geofluids 10, 406-421.

Bouwer, H., 1991. Simple Derivation of the Retardation Equation and Application to Preferential Flow and Macrodispersion. Ground Water 29(1), 41-46.

Dean, C., Reimus, P., Oates, J., Rose, P., Newell, D., Petty, S., 2015. Laboratory experiments to characterize cation-exchanging tracer behavior for fracture surface area estimation at Newberry Crater, OR. Geothermics 53, 213-224.

Diersch, H.-J., G., 2009. FEFLOW 5.4: finite element subsurface flow and transport simulation system. Reference manual. DHI-WASY Ltd., Berlin, Germany, 292 pp

Fossum, M. P., Horne, R. N., 1982. Interpretation of Tracer Return Profiles at Wairakei Geothermal Field Using Fracture Analysis. Geothermal Research Council Transactions 6, 261-264.

Freeze, R.A., Cherry, J.A., 1979. Groundwater. Prentice-Hall, Englewood Cliffs, NJ, 604 pp.

Ghergut, I., Behrens, H., Sauter, M., 2013a. Can Peclet numbers depend on tracer species? going beyond SW test insensitivity to advection or equilibrium exchange. In: Proceedings $38^{\text {th }}$ Workshop on Geothermal Reservoir Engineering, Stanford Univ. (CA), SGP-TR-198, 326335 .

Ghergut, I., Behrens, H., Sauter, M., 2013b. Single-well tracer push-pull test sensitivity to fracture aperture and spacing. In: Proceedings $38^{\text {th }}$ Workshop on Geothermal Reservoir Engineering, Stanford Univ. (CA), SGP-TR-198, 295-308.

Ghergut, I., Behrens, H., Sauter, M., 2014. Tracer-based Quantification of Individual Frac Discharge in Single-well Multiple-frac Backflow: Sensitivity Study. Energy Procedia 59, 235242 . 
Guimerà, J., Carrera, J. 1997. On the interdependence of transport and hydraulic parameters in low permeability fractured media. In: Hard Rock Hydrosystems (Proceedings of Rabat Symposium S2, May 1997). IAHS Publ. no. 241, pp. 123-133.

Haggerty, R., McKenna, S.A., Meigs, L.C., 2000. On the late-time behavior of tracer test breakthrough curves. Water Resources Research 36(12), 3467-3479.

Horne, R. N., Rodriguez, F. J., 1981. Dispersion in Tracer Flow in Fractured Geothermal Systems. In: Proceedings $7^{\text {th }}$ Workshop on Geothermal Reservoir Engineering, Stanford University (CA), SGP-TR-55, 103-107.

Horne, R.N., 1985. Reservoir Engineering Aspects of Reinjection. Geothermics 14, 449-457.

Huenges, E. (Ed.), 2010. Geothermal Energy Systems: Exploration, Development and Utilization, Weinheim:Wiley-VCH. 463pp.

Jung, Y., Pruess, K., 2011. A closed-form analytical solution for thermal single-well injectionwithdrawal tests. Water Resources Research 48(3), DOI: 10.1029/2011WR010979.

Karmakar, S. in preparation, Single-well tracer push-pull method development for characterizing subsurface processes. Ph.D. Thesis, University of Göttingen, Germany.

Karmakar, S., Ghergut, I., Sauter, M., 2015. EGS in sedimentary basins: sensitivity of earlyflowback tracer signals to induced-fracture parameters. Energy Procedia 76, 223-229. doi:10.1016/j.egypro.2015.07.906

Kinzelbach, W., Rausch, R., 1995. Grundwassermodellierung: Eine Einführung mit Übungen. Gebr. Borntraeger, Berlin Stuttgart, pp. 39-40.

Kocabas, I., Horne, R.N., 1987. Analysis of Injection-Backflow Tracer Tests in Fractured Geothermal Reservoirs. In: Proceedings $12^{\text {th }}$ Workshop on Geothermal Reservoir Engineering, Stanford University (CA), SGP-TR-109.

Maier, F., Kocabas, I., 2013. Efficient analytical solution for parameter estimation of push shut-in pull experiments in an idealized single fracture system. In: Proceedings $38^{\text {th }}$ Workshop on Geothermal Reservoir Engineering, Stanford University (CA), SGP-TR-198.

Mayerhofer, M.J., Meehan, D.N., 1998. Waterfracs: results from 50 Cotton Valley wells. Society of Petroleum Engineers Annual Technical Conference, Paper 49104, New Orleans, Louisiana, 27-30 September 1998.

Mayerhofer, M.J., Richardson, M.F., Walker, R.N.Jr., Meehan, D.N., Oehler, M.W., Browning, R.R.Jr., 1997. Proppants? We don't need no proppants. Society of Petroleum Engineers Annual Technical Conference, Paper 38611, San Antonio, Texas, 5-8 October 1997.

McDaniel, R.R., Holmes, D.V., Borges, J.F., Bajoie, B.J., Peeples, C.R., Gardner, R.P., 2009. Determining Propped Fracture Width from a New Tracer Technology. SPE paper 119545. SPE Hydraulic Fracturing Technology Conference, The Woodlands, Texas, USA, 19-21 January, 2009. 
Nottebohm, M., Licha, T., Ghergut, I., Nödler, K., Sauter, M., 2010. Development of Thermosensitive Tracers for Push-Pull Experiments in Geothermal Reservoir Characterization. In: Proceedings World Geothermal Congress Bali, Indonesia), 6pp.

Pruess, K., Bodvarsson, G.S., 1984. Thermal Effects of Reinjection in Geothermal Reservoirs with Major Vertical Fractures. J. of Petroleum Technology 36, 1567-1578.

Pruess, K., Doughty, C., 2010. Thermal single-well injection-withdrawal tracer tests for determining fracture-matrix heat transfer area. In: Proceedings $35^{\text {th }}$ Workshop on Geothermal Reservoir Engineering, Stanford University (CA), SGP-TR-188.

Pruess, K., 2002. Numerical Simulation of Multiphase Tracer Transport in Fractured Geothermal Reservoirs. Geothermics 31, 475-499.

Rose, P., Mella, M., Kasteler, C., Johnson, S.D., 2004. The Estimation of Reservoir Pore Volume from Tracer Data. In: Proceedings $29^{\text {th }}$ Workshop on Geothermal Reservoir Engineering, Jan. 26-28, 2004, Stanford Univ. (CA), SGP-TR-175.

Rose, P., Leecaster, K., Clausen, S., Sanjuan, R., Ames, M., Reimus, P., Williams, M., Vermeul, V., Benoit, D., 2012. A Tracer Test at the Soda Lake, Nevada Geothermal Field Using a Sorbing Tracer. In: Proceedings $37^{\text {th }}$ Workshop on Geothermal Reservoir Engineering, Jan. 30 - Feb. 1, 2012, Stanford Univ. (CA), SGP-TR-194.

Rose, P.E., Xu, T., Fayer, S., Pruess, K., 2010. Chemical Stimulation for EGS: the Use of Chelating Agents at High pH for the Simultaneous Dissolution of Calcium Carbonate, Silicas, and Silicates. In: Proceedings World Geothermal Congress, Bali, Indonesia, 04/2010, 1-11

Schroth, M.H., Istok, J.D., Haggerty, R., 2001. In situ evaluation of solute retardation using singlewell push-pull tests. Advances in Water Resources, 24, 105-117.

Singhal, B.B.S., Gupta, R.P., 2010. Applied Hydrogeology of Fractured Rocks (2nd ed.). Springer $408 \mathrm{pp}$.

Tomac, I., Gutierrez, M., 2015. Micromechanics of proppant agglomeration during settling in hydraulic fractures. J. Petrol. Explor. Prod. Technol. 151, DOI 10.1007/s13202-014-0151-9.

Tsang, Y.W., 1992. Usage of 'Equivalent Apertures' for Rock Fractures as Derived From Hydraulic and Tracer Tests. Water Resour. Res. 28(5), 1451-1455.

Zimmermann, G., Moeck, I., Blöcher, G., 2010. Cyclic waterfrac stimulation to develop an enhanced geothermal system (EGS): Conceptual design and experimental results. Geothermics 39, 59-69. 
CHAPTER 2-PART 2: EGS in sedimentary basins: sensitivity of early-flowback tracer signals to induced fracture parameters

Shyamal Karmakar*, Julia Ghergut, and Martin Sauter

Citation:

Karmakar, S., Ghergut, I., Sauter, M., 2015. EGS in sedimentary basins: sensitivity of early flowback tracer signals to induced-fracture parameters. Energy Procedia 76, 223-229. doi:10.1016/j.egypro.2015.07.906

Geoscience Centre of the University of Göttingen, Department of Applied Geology, Goldschmidtstrasse 3, 37077 Göttingen, Germany

*Corresponding author: shyamal.karmakar@geo.uni-goettingen.de 


\begin{abstract}
Short-term flowback signals from injection-flowback tracer test face a certain degree of ambiguity in fracture parameter inversion from the measured signal of a single tracer. This ambiguity can be overcome by combining different sources of information (lithostratigraphy, and hydraulic monitoring) and concomitantly using several tracers with different transport behavior. To improve the early-time characterization of induced fractures, of either gelproppant or waterfrac, we recommend using tracers of contrasting sorptivity to rock surfaces, and to proppant coatings where applicable. We illustrate the application of such tracer combinations using hydro- and lithostratigraphy data from the Geothermal In-situ Laboratory at Groß Schönebeck, Germany.
\end{abstract}




\title{
1 Introduction
}

Artificial-fracture design, and fracture characterization during or after stimulation treatment is a central aspect of many enhanced geothermal system (EGS) of both in gel-proppant (GPF) or in water fracture (WF) type stimulation projects. Tracer testing is a standard method of determining mass transport within a geothermal reservoir and can be a valuable tool in the design and management of production and injection operations (Horne 1985, Pruess, 2002). Various single well (SW) tracer test approaches had revealed a great promise for parameter estimation in fractured georeservoir using characteristic discharge rate, ion exchange (Dean et al 2015) as well as a sorptive tracer. Late-time tracer signals from SW injection-flowback tests have been used mainly for inflow profiling for EGS developed in multi-zone reservoirs in the NE-German basin (Ghergut et al 2014). However, the potential of using early flowback tracer signal from SW test for fracture characterization remained unexplored so far. Furthermore, no 'effort-against-benefit' analysis has been undertaken in a more focused manner, leaving issues like the above as a matter of subjective pondering. We explored and outlined the actual benefit from early-flowback sampling and from using more than one tracer per fracture. Additionally, what improvements to parameter sensitivity can we expect? Moreover, this paper sought the answer for early-sampling frequency as a trade-off between 'too much effort' and 'too sparse information', especially for the case of gel-proppant fractures (GPF), where early flowback sampling is likely to pose greater difficulties. In this instance, this paper aims to provide greater insight into the early time tracer signal- based parametric study for fracture characterization in an EGS of deep crystalline and/or sedimentary formations.

\section{Nomenclature}

\author{
SW Single-well \\ GPF Gel-proppant fracture \\ WF Water fracture \\ PST Proppant sorptive tracer \\ MST Matrix sorptive tracer \\ $\mathrm{k} \quad$ Sorption coefficient, a multiple of Henry sorption coefficient $\left(K_{d}\right)$ and density
}




\section{Gel proppant fracture and water fracture simulation parameters}

In our three-dimensional discrete fracture reservoir model in Feflow5.4 (Diersch 2009) we used lithostratigraphic and hydrogeological data of Groß Schönebeck, NE German basin (Blöcher et al. 2010) at depths of $-3968 m$ to $-4004 m$ (sedimentary formation, $3 \%$ porosity, hydraulic conductivity $6.4 \times 10^{-9} \mathrm{~ms}^{-1}$ ) for the gel proppant fracture, and from $-4147 \mathrm{~m}$ to $-4300 \mathrm{~m}$ for the water fracture reservoir (volcanic formation, $0.5 \%$ porosity, hydraulic conductivity $3.2 \times 10^{-9}$ $\mathrm{ms}^{-1}$ ). The horizontal extent of a model domain defined as twice the maximum tracer travel distance and the depth was implemented toward the y-axis. Tracer was injected as a short pulse both in the gel-proppant and water fracture simulation. The chaser fluid was injected after pulse injection to drag the tracer up to $\leq$ half fracture height. We conducted simulations following forward modeling principle using fracture thickness values $2 \mathrm{~mm}$ to $24 \mathrm{~mm}$ and fracture porosity $30 \%$ to $60 \%$ for gel-proppant treatment fracture and fracture aperture $0.1 \mathrm{~mm}$ to $1.44 \mathrm{~mm}$ for water fracture (Huenges 2010) for the solute transport with different sorptivities, k ( $\mathrm{k}=$ Henry sorption coefficient, $K_{d} \times$ density). Two different sorptive tracers were used for gel-proppant fracture viz. matrix sorptive or proppant sorptive. Injection volume estimated as $\sim$ half of the fracture volume for both water fracture and gel-proppant fracture while injection duration and rate remained unchanged. Sorptive tracer/s, together with a conservative tracer, was/were coinjected as a short pulse in water fracture or gel-proppant fracture. Fracture flow in gel-proppant fracture was treated as Darcy flow and for water fracture, cubic law for flow in the fracture and Darcian flow in the matrix.

\section{Conservative and sorptive tracer test in gel proppant fracture}

\subsection{Conservative tracer}

Early time flow-back tracer signal showed higher tracer peak, for lower dispersion. Matrix parameter and fracture porosity or different packing of proppant do not affect tracer signals significantly. From this analogy, it showed that conservative tracer would be effective to estimate dispersion of solute in fracture (Figure 2.2.1 and 2.2.2). 


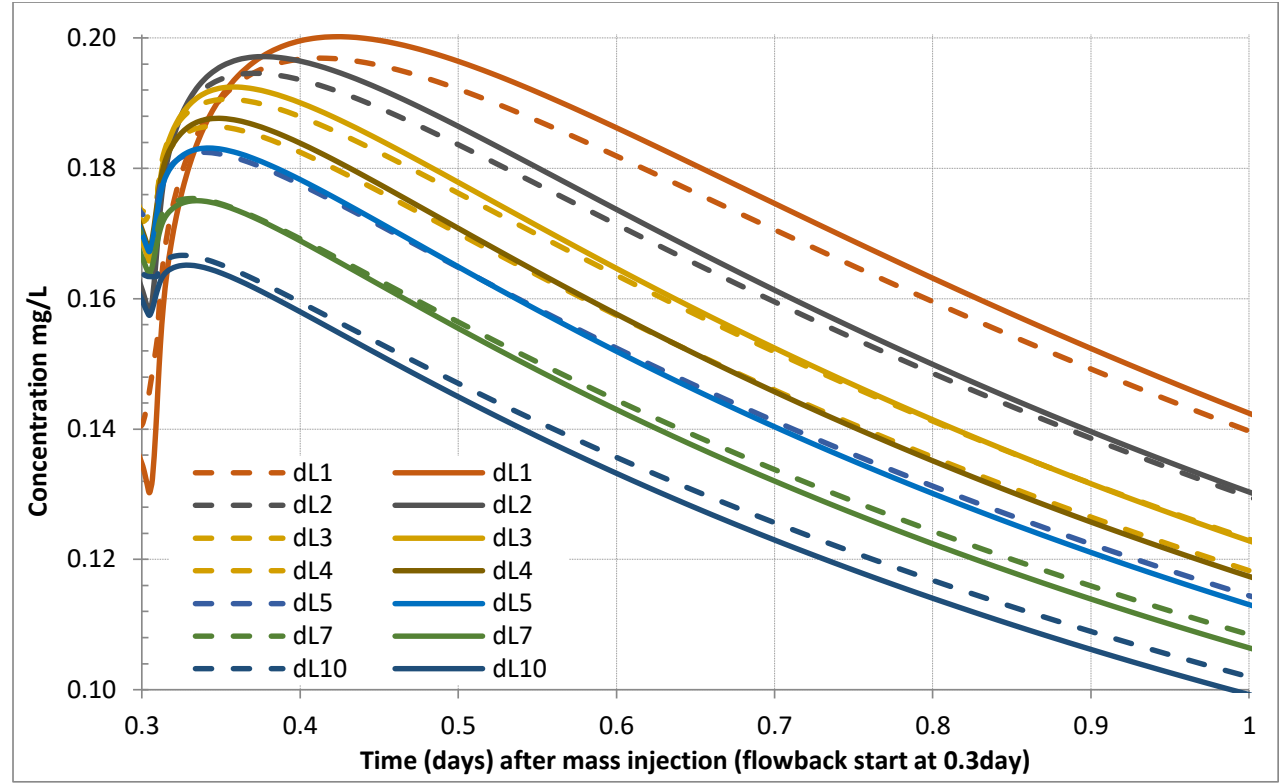

Figure 2.2.1: Conservative tracer flowback signals from different dispersivity and fracture thickness: broken line- $4 \mathrm{~mm}$, solid line- $16 \mathrm{~mm}$

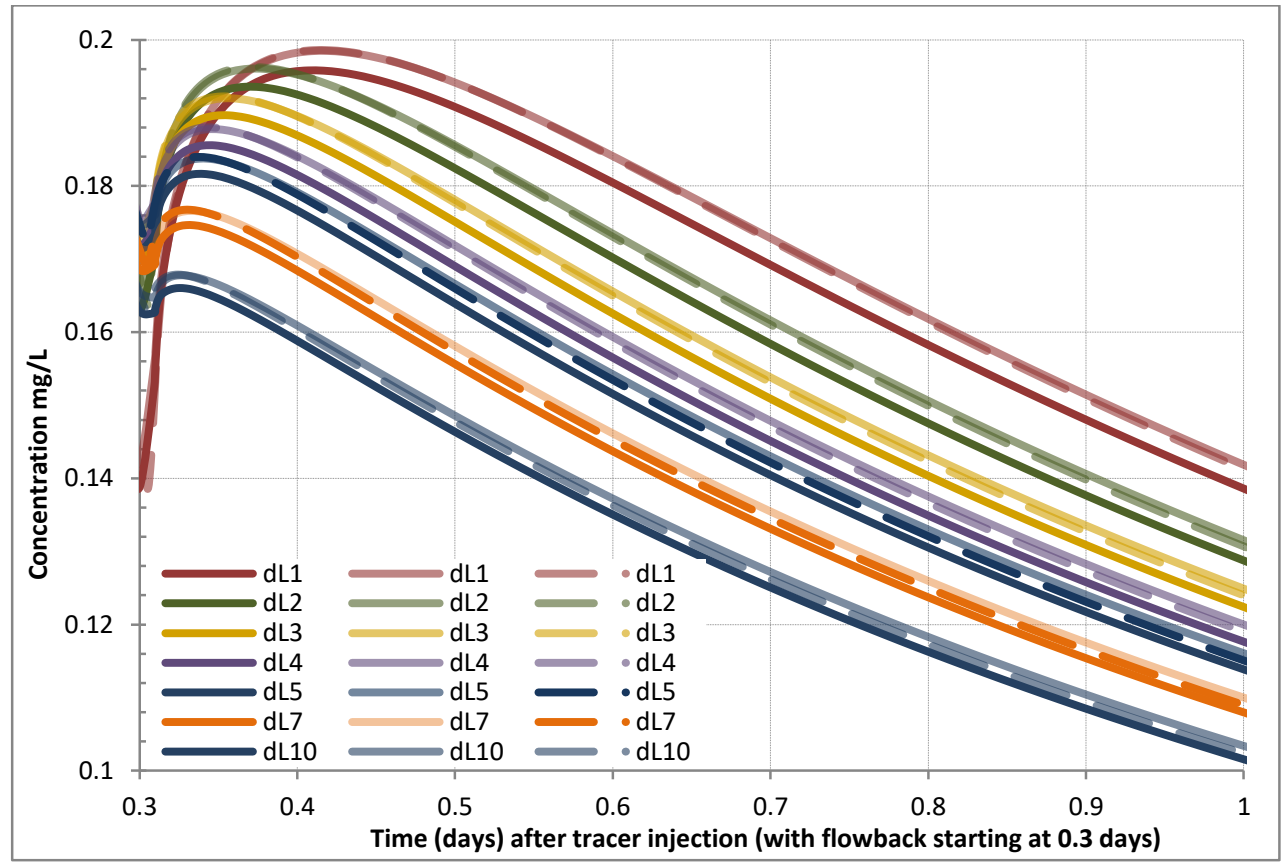

Figure 2.2.2: Conservative tracer flowback signals from different dispersivity and fracture porosity. 


\subsection{Matrix sorptive tracer}

The simulations result of matrix sorptive tracer (MST) revealed that early flowback tracer signal could characterize fracture thickness (Fig. 2.2.3). However, tracer sensitivity was not increased proportionally with increasing tracer sorptivity on matrix. The flowback tracer signal from the bigger fracture thickness showed higher consistency for a longer sampling period than the smaller fracture thickness. The sensitivity with fracture thickness would increase with increasing sorptive properties on matrix. However, in very early time tracer signal from low sorptive tracers, the tracer signal is conversing very early for different fracture thickness suggest that very low sorptive tracer uses would not ideal for this estimation. Furthermore, very high sorptive tracer on matrix would not produce higher efficiency or higher sensitivity because it will see very small part of the fracture and matrix.

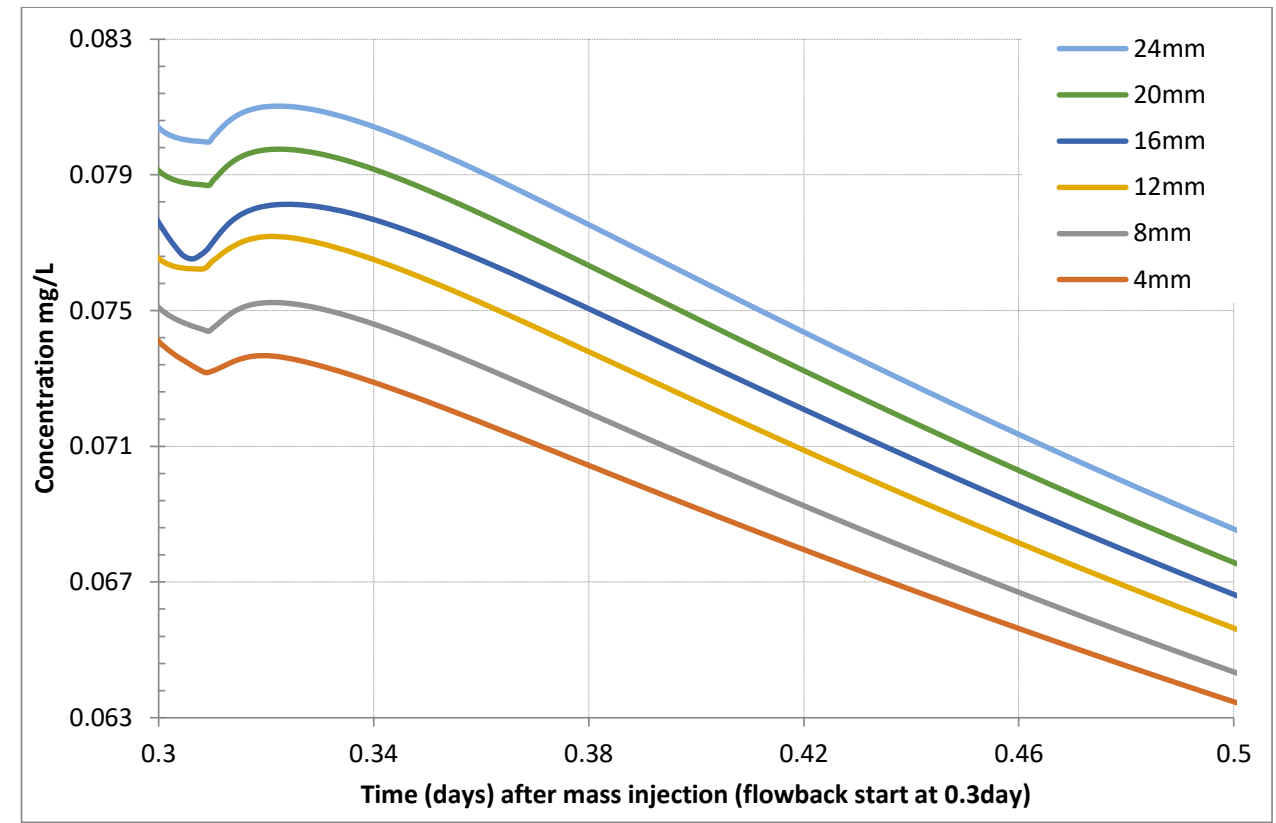

Figure 2.2.3: MST ( $k=1.5)$ tracer concentrations resulting from a GPF treatment with fracture porosity $55 \%$.

The early time tracer signal from injection-flowback tests showed lower peak and higher tail tracer concentration with the decrease of fracture thickness (Fig. 2.2.3). MSTs, with medium to higher sorption value, can unambiguously identify different fracture thickness within the first day of flowback testing. Moreover, with increasing test duration the tail from the stronger peak depletes quickly while the low peak from a lower fracture thickness showed delayed tracer release and stronger tracer concentration. Hence, for early time tracer injection-flowback test, it is advisable to apply a medium ranged $(0.7<\mathrm{k}<2.5$, matrix porosity $3 \%)$ matrix sorptive tracer. 
The good news is that the MST did not show any influence from fracture porosity. In our simulation, we find the same tracer signals from different fracture porosity with same fracture thickness. Therefore, the MST can unambiguously estimate fracture thickness. This fracture thickness estimate can be used to determine fracture porosity from the proppant sorptive tracer breakthrough curve for a specific fracture.

\subsection{Proppant sorptive tracer}

The early-time tracer signal can effectively estimate either fracture thickness or fracture porosity using a proppant sorptive tracer (k-value 25 to 80). Fig. 2.2.5 illustrates the ambiguity of early tracer signal from the flowback test in GPF over two parameter fracture thickness and fracture porosity. From simulation result, it also revealed that a weak sorptive tracer $(\mathrm{k}<25)$ would not produce an effective early-flowback tracer signal to evaluate fracture thickness or porosity. Highly sorptive tracers $(\mathrm{k}>80)$, for obvious reasons, see the very small part in the fracture after the borehole. From PSTs flowback concentration results, it is evident that early time tracer signals would be useful for medium retarded tracer with a retardation factor $25<\mathrm{k}<80$, to avoid ambiguity due to the error in detection and error in sampling and extrapolation. The monotonicity of tracer concentration from different fracture thicknesses revealed that the effect would prevail longer than the first day after injection while increasing porosity; the sensitivity would gradually increase for a decrease in fracture thickness (Fig. 2.2.4). Both sensitive parameters (here fracture porosity and fracture thickness) for the proppant sensitive tracer could be ambiguous to distinguish from a single test/tracer. 


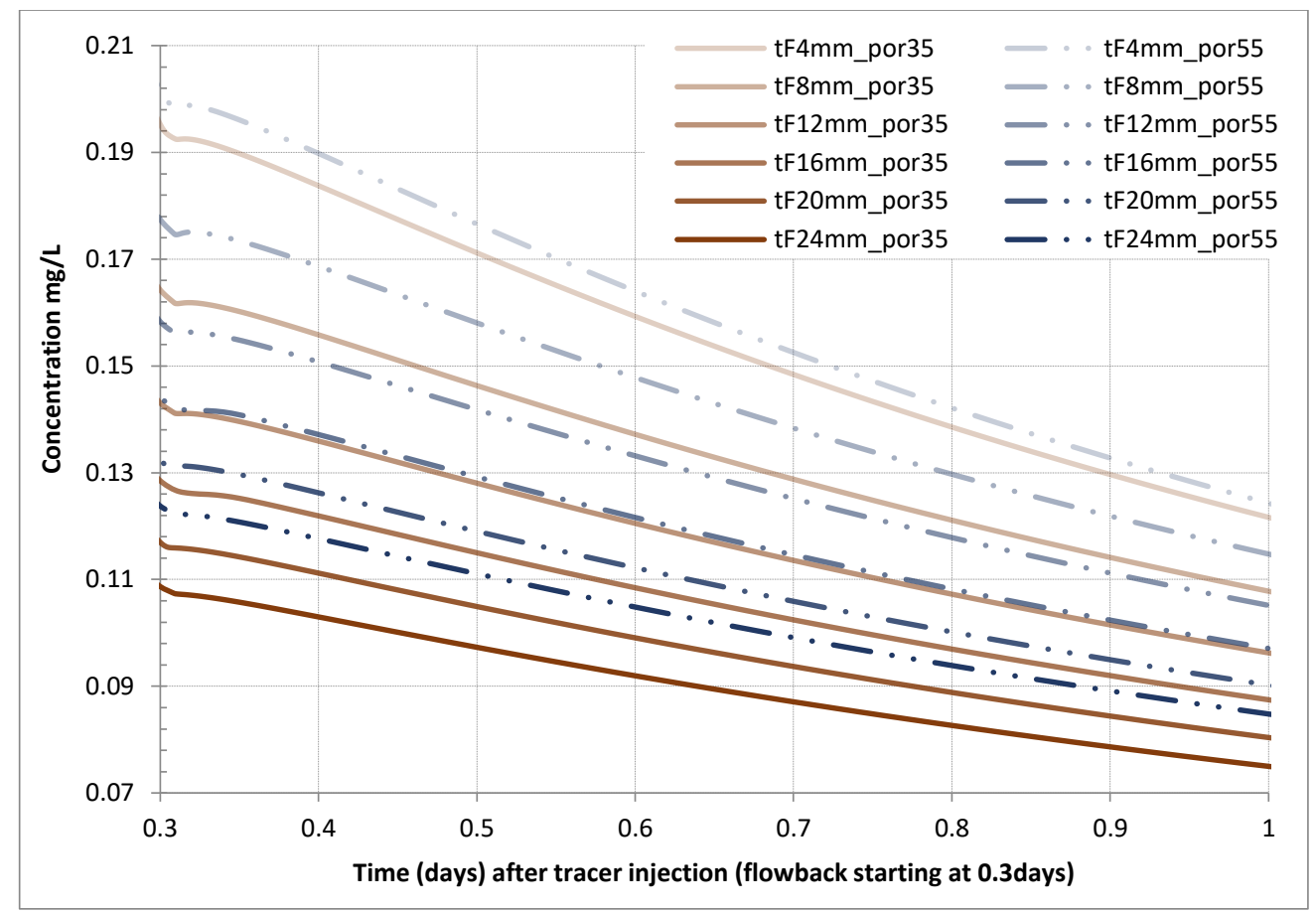

Figure 2.2.4: PST $(\mathrm{k}=40)$ flowback concentration for porosity (por) $35 \%$ and $55 \%$ for fracture thickness $(\mathrm{tF})$ in gel-proppant fracture.

Figure 2.2-5 and 2.2-6 show different tracer injection duration flowback signals. From this result, it revealed that smaller injection duration (here 0.3days) would produce stronger tracer signal from both in different fracture thickness and porosity than a longer injection duration (here 0.8 days).

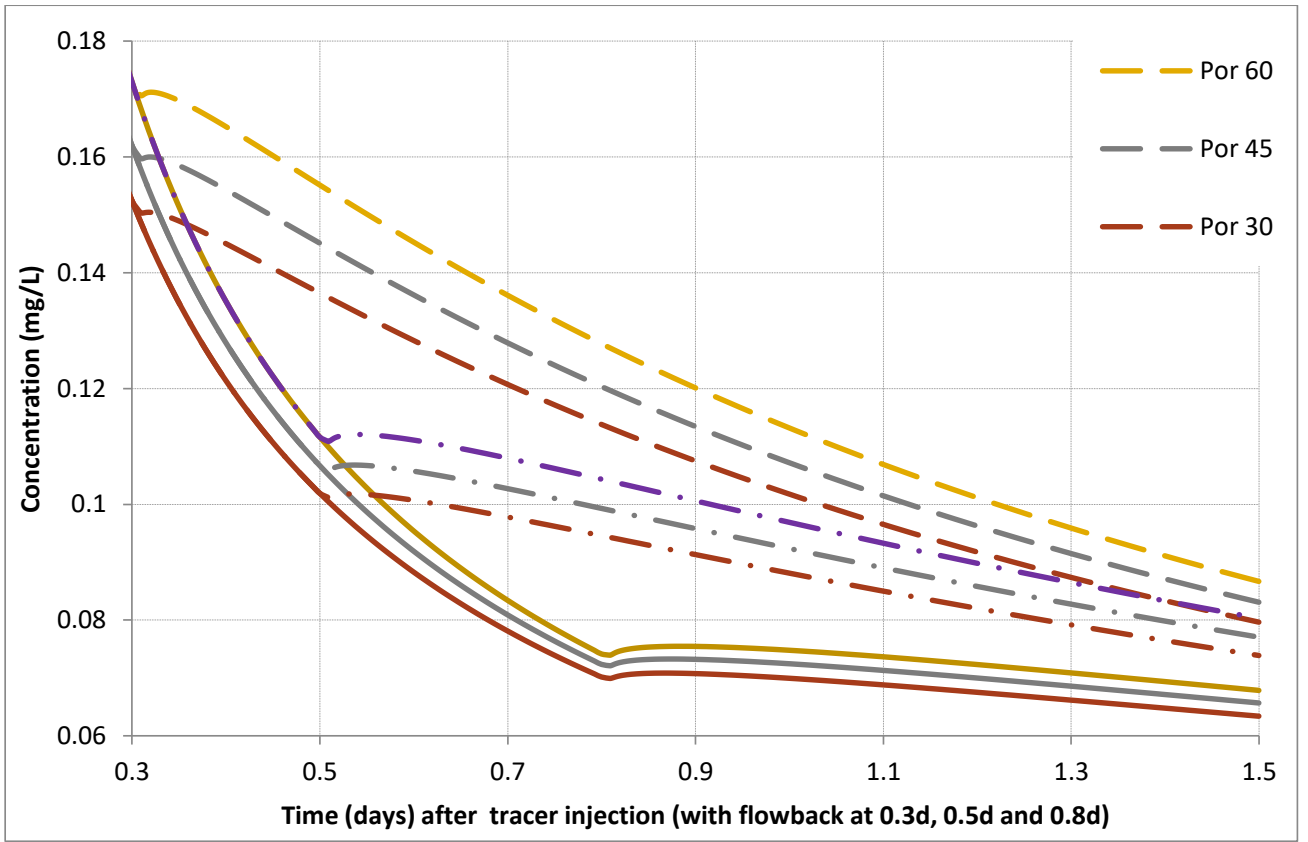


Figure 2.2.5: Tracer injection duration effect on flowback proppant sorptive tracer signals for different fracture porosity.

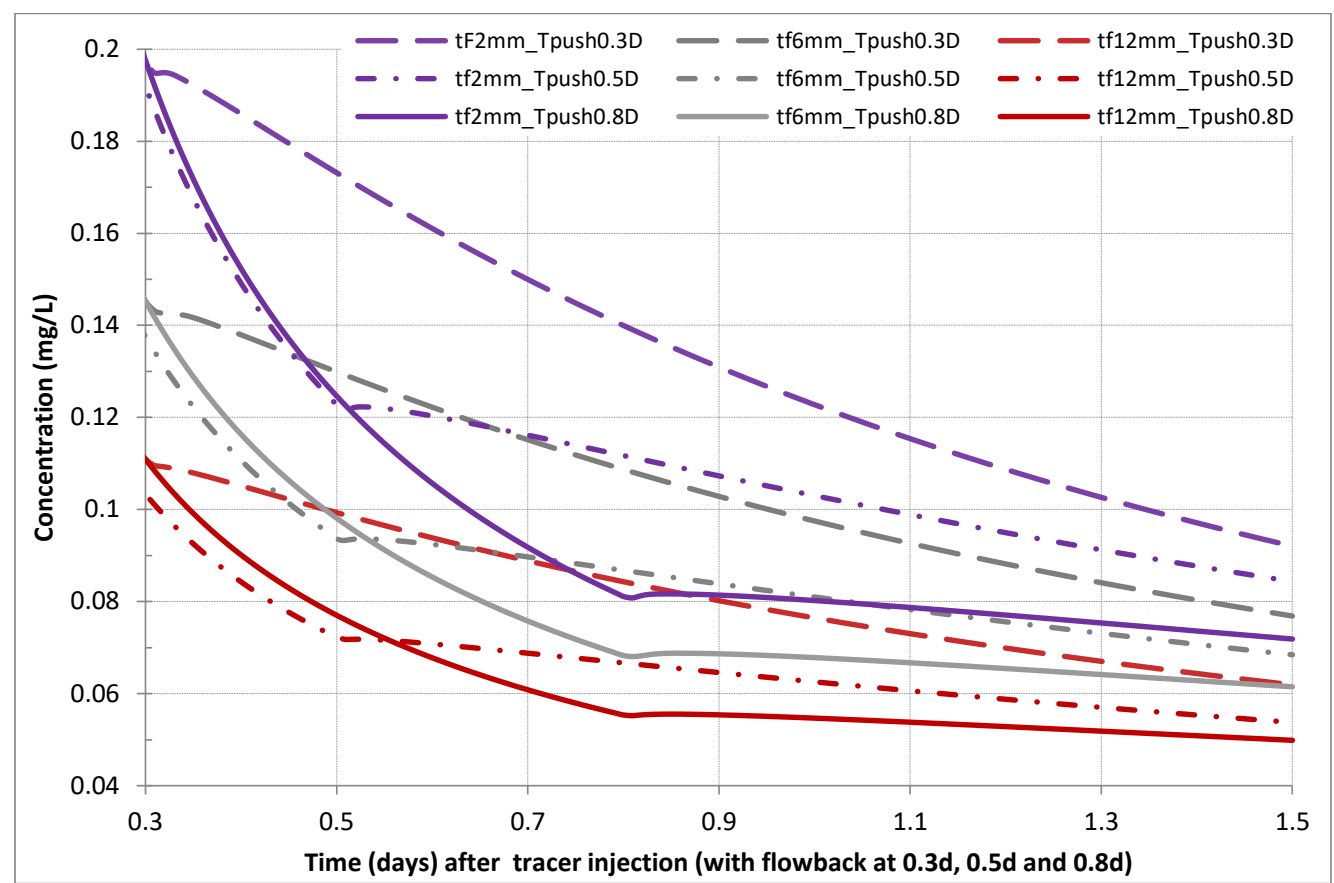

Figure 2.2.6: Tracer injection duration effect on flowback proppant sorptive tracer signals for different fracture thickness.

\section{Conservative and sorptive tracer test in water fracture}

\subsection{Conservative tracer}

We considered using diffusive tracer with different molecular diffusion properties to inject during SW tracer test in water fracture. However, the flowback tracer signal showed no significant variation with fracture aperture for different diffusive tracers. Conservative tracer signals varied significantly for different fracture aperture (Fig. 2.2.7). 


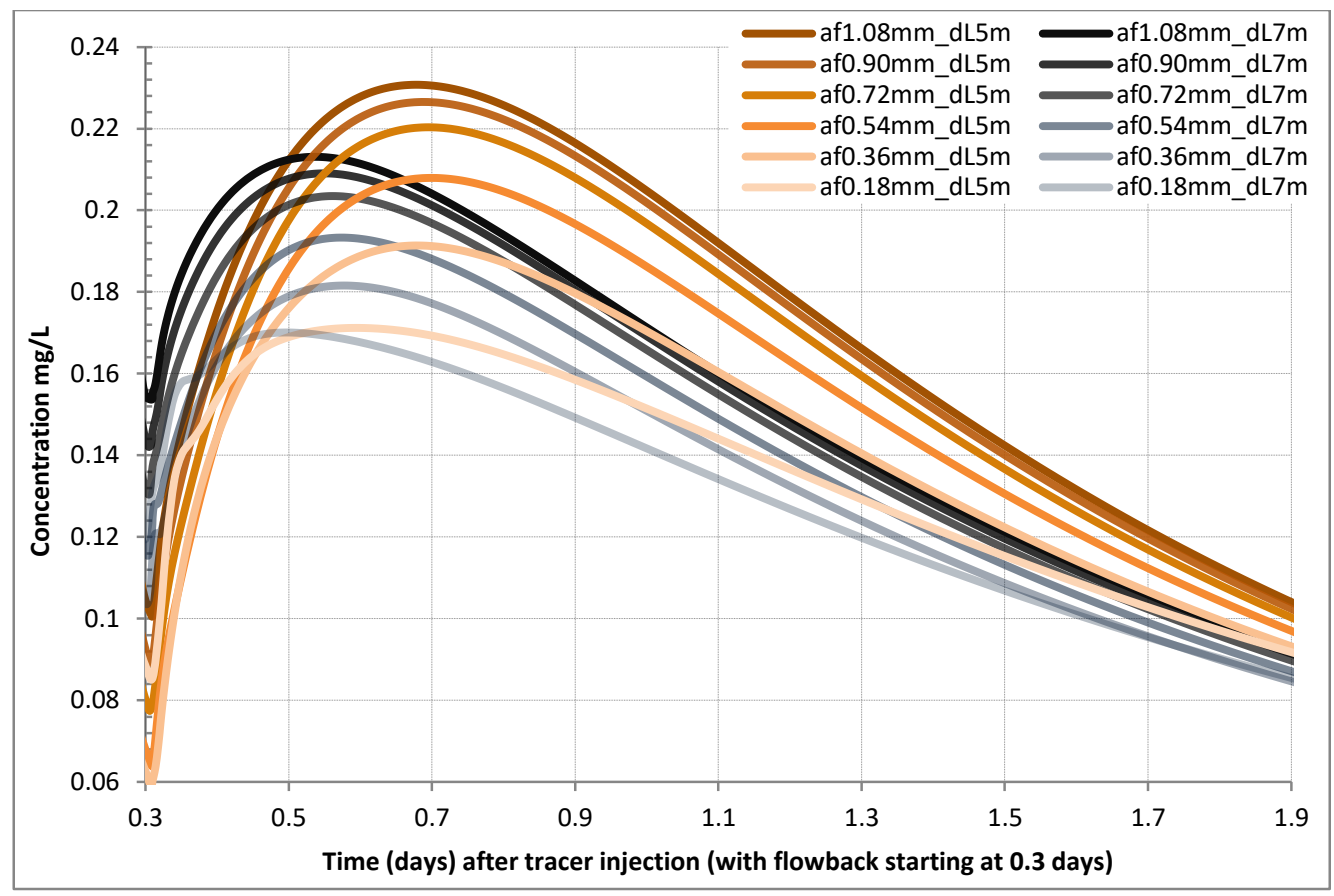

Figure 2.2.7: Conservative tracer flowback signals for different fracture aperture (af) and dispersion length $(\mathrm{d} L)$ in WF treatment.

\section{Summary and conclusions}

The early tracer signal can produce enough data to characterize fracture thickness and porosity while using or sacrificing two tracers that are retarded only by either the proppant or matrix. Ambiguity with fracture aperture and the fracture porosity for PST in GPF treatment can be overcome by using a matrix sorptive tracer (MST) simultaneously. The MST is independent to the fracture porosity. From the above simulation results, we can recommend a tracer application scheme to evaluate stimulated fracture thickness and porosity. However, the effectiveness needs to be verified for very thin GPFs $(\leq 2 \mathrm{~mm})$. Early time tracer signal from conservative solute can determine the fracture aperture for water fractures.

Again, low sorption properties tracer would limit matrix sorptive tracers' use. Variable injection rates were found to be effective during water fracture stimulation rather a constant flow rate (Zimmermann et al 2010). Hence, this early time tracer- based monitoring would be especially suitable to integrate during any stage of reservoir lifetime (EGS development, treatment, and engineering). Summarizing, our simulation effectively produced two significant results. Firstly, the water fracture aperture can be effectively evaluated by using the early-time tracer signal of a conservative tracer. Secondly, by using the combination of MST and PSTs', we can accurately determine fracture thickness and porosity in gel proppant fractures from a single test. Lastly, the small radial scale of such tests, i.e., small fluid turnover volume, and 
thus little dilution of the injected tracers, has three practical advantages: (1) one does not need to inject large tracer quantities, (2) one does not need to wait, sample and quantify signal tailings, (3) the field-site detection and/or laboratory-instrumental metering of tracer signals need not be conducted in the low-level range.

\section{Acknowledgements}

This work benefited from long-term support from Baker Hughes (Celle) and from the Lower Saxonian Science and Culture Ministry (MWK Niedersachsen) within the applied research project gebo (Geothermal Energy and High-Performance Drilling, 2009-2014), and from the Federal German Ministry for Economic Affairs and Energy (BMWi) within the project TRENDS (FKZ 0325515). The first author gratefully acknowledges continued financial support from the DAAD (German Academic Exchange Service) to pursuing his Ph.D. work.

\section{References}

Horne RN. 1985, Reservoir engineering aspects of reinjection. Geothermics 14(2/3):449-57.

Pruess K. 2002, Numerical simulation of multiphase tracer transport in fractured geothermal reservoirs. Geothermics 31(5): 475-99.

Dean C, Reimus P, Oates J, Rose P, Newell D, Petty S. 2015, Laboratory experiments to characterize cation-exchanging tracer behavior for fracture surface area estimation at Newberry Crater, OR. Geothermics 53:213-24.

Ghergut I, Behrens H, Sauter M. 2014, Tracer-based quantification of individual frac discharge in single-well multiple-frac backflow: sensitivity study. Energy Procedia 235-42.

Diersch HJG 2009. FEFLOW Finite element subsurface flow and transport simulation system User's [2] Manual/Reference Manual/White Papers, Release 5.4. Berlin: WASY Ltd.

Blöcher G, Zimmermann G, Moeck I, Brandt W, Hassanzadegan A, Magri F. 2010, 3D numerical modeling of hydrothermal processes during the lifetime of a deep geothermal reservoir. Geofluids 10(3): 406-21.

Huenges E. (Ed.), 2010, Geothermal energy systems: Exploration, development and utilization, Weinheim: Wiley-VCH.

Zimmermann G, Moeck I, Blöcher G, 2010, Cyclic waterfrac stimulation to develop an enhanced geothermal system (EGS): Conceptual design and experimental results. Geothermics 39(1):59-69. 
CHAPTER 2-PART 3: Early time flowback tracer test for stimulated crystallinegeoreservoir of multiple parallel fracture characterization

Shyamal Karmakar*, Iulia Ghergut, and Martin Sauter

Citation:

Karmakar, S., Ghergut, I., Sauter, M., draft manuscript, Early time flowback tracer test for stimulated crystalline-georeservoir of multiple parallel fracture characterization, Prepared for next Stanford Geothermal workshop, 2017

Geoscience Centre of the University of Göttingen, Department of Applied Geology,

Goldschmidtstrasse 3, 37077 Göttingen, Germany

*Corresponding author: shyamal.karmakar@geo.uni-goettingen.de 
Keywords: sorptive tracer, conservative tracer, EGS, HDR, fracture aperture, fracture porosity, dispersivity

\begin{abstract}
Stimulated georeservoir needs to deal with the fracture properties of which, most of them are estimated from geological inherent knowledge, hydraulic test and geophysical methods except transport effective parameters such as fracture aperture and effective porosity. Single-well tracer injection-flowback based 'early time' tracer signal provides a relatively small duration and efficient test method to estimate those transport effective parameters, that is relevant not only for EGS of sedimentary formation but also other stimulated geo-reservoirs (e.g. HDR or oil/gas reservoirs). The application that described in early-time flowback tracer test study article has not exhaustively demonstrated its complete range of uses for stimulated georeservoir. Sorptive tracer either on proppant or on a matrix that used for stimulated fracture characterization has raised the question about the range of sorptive tracer to produce for an effective tracer test that is very important to know beforehand conducting such a field experiment. This study accounts the full array of sorptive tracers for different candidate georeservoir and at the same time a guideline for candidate tracer in relation to georeservoir properties proposed. For the purposes of the present study, a lower sorptive tracer than its minimum necessary was suggested, assuming a sensitivity improvement factor (ratio between sorptive tracer signal changes to conservative tracer signals changes, s/c) approximately equal to $\sqrt{ }(1+0.7 \times$ sorption coefficient, $\kappa)$. One needs to note that the higher the tracer's retardation, the lower is its fracture invasion, and consequently a poorer capability for characterizing the fracture as a whole. In principle, this could be compensated by increasing the chaser volume (i.e., by injecting sorptive tracers earlier than conservative tracers).
\end{abstract}




\section{Introduction}

Over the years, different definitions of EGS (Schulte et al. 2010, Breede 2013 and others) have been proposed, covering a broad variety of formation, depth, temperature, reservoir permeability and porosity, type of stimulation technique involved, etc. Therefore, typical geological settings for EGS includes a range of formation covering igneous (e.g. Iceland), metamorphic (e.g. Lardarello, Italy), magmatic (e.g. Soultz, France) and sedimentary (e.g. Groß-Schönebeck and Horstberg, Germany) formations. The hot dry rock (HDR) type of geothermal energy can play a vital role in all the different forms of geothermal energy and estimated that more than $90 \%$ of the total accessible geothermal energy (Jiang et al. 2014). The HDR system recovers the earth's heat via closed-looped circulation of fluid from the surface through a man-made, confined reservoir several kilometers deep. The technology bears little similarity to that of the hydrothermal industry and carries a worldwide applicability hence it claims as ubiquitous (Breede 2013). Recently, Jung (2013) has reconstructed the background to 'contemporary EGS', from the original HDR concept based on multi-zone hydraulic fracturing in crystalline formations to a multiple wing crack model in naturally fractured crystalline formations.

Early-time tracer injection flowback test that relies on the tracer signal during very early phase of flowback and sampling even for less than a day after injection can determine fracture porosity and fracture thickness/aperture in the realm of stimulated fracture georeservoir (Karmakar et al. 2015a, 2015b). In last two part (chapter 2, part 1 and 2), studies have discussed proppant and matrix sorptive tracers that can be used to characterize fracture aperture, fracture porosity and dispersivity in stimulated fracture of enhanced geothermal system (EGS) both in sedimentary formation and crystalline. However, the sensitivity regime of single-well tracer test as described in Schroth et al. 2001, Ghergut et al., 2013 have included fracture aperture and dispersion in the sensitive parameter structure as both of this parameter affect flowback tracer signal affect similar manner (cf. Karmakar et al. 2015b). Fracture length as an unknown parameter in multiple parallel fracture model as proposed and discussed in many current studies (Jung 2013, Zau et al. 2015 and others), the early-time tracer flowback signal would be embroidered with the extend fracture surface area. This article highlights the use of early time tracer flowback signal for fracture length characterization in hot, dry rock (HDR) system EGS where single fracture represents a limited number of parallel fracture stimulated on the same plane. And for our simulation study, we refer the 'HDR type EGS' as the multiple parallel 
fractures in tight rock formation stimulated hydraulically with a minuscule aperture with a particular fracture spacing.

\section{Model concept and parameter selection}

A conceptual model for stimulated fracture parameter estimation using single-well tracer method founded on the lesson learned from several tracer study in Northern and Southeast German sedimentary basin. Ghergut et al (2013) have demarcated main ten parameters in the transport equation 1-7 initial-boundary value problems (IBVP) for single-well tracer test. It includes two fracture geometrical parameters (fracture aperture ' $b$ ' and fracture spacing ' $a$ ' in parallel fracture system), five hydrogeological properties (matrix porosity, matrix diffusion coefficients, longitudinal dispersivity within fracture, "aquifer" thickness, hydraulic diffusivity), and three SWPP test design variables, pull phase duration, injection and extraction rates or volumes.

$$
\begin{array}{r}
\frac{\partial C}{\partial t}+\frac{Q}{2 \pi B_{e f f}} \frac{\partial C}{r \partial r}-\frac{\alpha|Q|}{2 \pi B_{e f f}} \frac{\partial^{2} C}{r \partial r^{2}}-\left.\frac{\varphi_{m} D_{m}}{b} \frac{\partial C}{r \partial y}\right|^{y=a}=0 \\
\frac{\partial C_{m}}{\partial t}-D_{m} \frac{\delta^{2} c_{m}}{\delta y^{2}} \underbrace{D_{m} \frac{\delta^{2} c_{m}}{\delta r^{2}}}=0 \ldots \ldots \ldots \ldots(2)
\end{array}
$$

Choose to ignor

$$
\begin{aligned}
& \text { Initial conditions: } C(t=0, r)=0, C_{m}(t=0, r, y)=0 \ldots \ldots \ldots \ldots \\
& \text { Boundary conditions: } v C-\left.D_{h y d} \cdot \nabla C\right|_{r=0}=\text { Tracer flux }(t) \\
& \qquad v_{m} C_{m}-\left.D_{h y d} \cdot \nabla C_{m}\right|_{r=0} \approx 0 \ldots \ldots \ldots \ldots(5) \\
& \left.C(t, r \rightarrow \infty, y) \rightarrow 0) C_{m}(t=0, r \rightarrow \infty, y) \rightarrow 0\right) \ldots \ldots \ldots \ldots \ldots \\
& C_{m}(t, r, y=b)=C(t, r),\left.\quad \frac{\partial C_{m}(t, r, y)}{\partial y}\right|^{y=a}=0 \ldots \ldots \ldots
\end{aligned}
$$

Here $D_{\text {hyd }}\left[\mathrm{LT}^{-2}\right]$ - Hydrodynamic dispersivity in fracture or in matrix, $B_{\text {eff }}$ or b $[\mathrm{L}]$ is the total fracture thickness for gel-proppant fracture or fracture aperture for water-fracture (Tang et al 1992), density of fluid, $\rho_{0}\left[\mathrm{ML}^{-3}\right]$, source or sink of fluid or solute $\mathrm{Q}\left[\mathrm{L}^{3} \mathrm{~T}^{-1}\right] . C_{m}\left[\mathrm{ML}^{-3}\right]$, solute in matrix, $v_{m}\left[\mathrm{LT}^{-1}\right]-$ Darcy velocity in matrix

One-eighth of fracture-matrix volume assumed suffice to model due to considering the symmetry of fracture axis perpendicular to the injection well with a planar fracture (Ghergut et al., 2013b for parallel-fracture systems). The partial differential equation of linear flow and 
transport equation are solved the IBVPs numerically by using a commercial finite element software, FEFLOW analyzing the output (i.e., the simulated tracer signals) regarding sensitivity to target parameters and of parameter interplay, as applicable). Though all hydrogeological parameters are of distributed (local) type, and their values may change with time by virtue of coupled THMC processes (as induced by SWPP-forced hydraulic and thermal gradients). This implies that a virtually infinite number of degrees of freedom. One global value for each parameter is assumed during the simulation study, i.e. a spatially homogeneous system whose properties do not change with time (A brief discussion on it outlined in Ghergut et al. 2006, 2011, 2013).

\subsection{Fracture model for injection-flowback test}

To understand parameter estimation of the early-time SWIF method in HDR type EGS, it is necessary to re-define the ratio of fracture volume vs. injection volume because injected tracer (while it is very low sorptive) will flow out the fracture while the ratio is $<1$. A vertical cluster of fractures, here six fracture with equal spacing between $40 \mathrm{~m}$ to $160 \mathrm{~m}$ in a granite formation is considered. However, symmetry along the horizontal injection well and remoteness between two adjacent parallel fracture-matrix have assumed for solute tracer transport . Hence, it is found that $1 / 8$ of single fracture with the assumption of no-gravitation effect valid for the model (Fig 2.3.1). The half of fracture (b) is implemented using 'discrete feature element' of Feflow 5.4 (Diersch 2009) by choosing 'Hagen-Poiseuille's ('cubic law') flow assuming a linear flow. The transport equation (PDEs) contains an oblique boundary condition for the IBVP. Injection borehole expected to be placed in the middle of the fracture. This alignment will facilitate maximum circulation of fluid as well as energy output. The distance between injection and production well assumed as 300-500m following common HDR type EGS of the world. For our study, we took a standardized case as a set of effective fracture aperture and matrix porosity and permeability combination for volcanic formation. We considered different fracture spacing and fracture length in multiple oblique 2D fractures on a 3D matrix domain that discretized into eight-nodded quadrilateral prism elements. To be noted here, all three mechanisms relate to one major process is matrix sorption on the fracture surface and inside the matrix; no further process (like matrix diffusion, dispersion, reactions, etc.) has considered in this study. However, for the high sorptive tracer, exchange at the fracture boundary can be ignored as tracer will be sorbed at the fracture surface at the immediate vicinity of the well or need very long tracer injection duration as assumed after Schroth et al. (2001), Ghergut et al. (2013) and Karmakar et al (2015) anlysis with retardation tracer. Therefore, early time tracer signals 
sensitivity was investigated over four injection volume regime $\left(1 / 3,1 / 2,3 / 4\right.$ and $1 \frac{1}{2}$ of fracture volume) using same injection rate while varying the injection duration only. The matrix parameter set consist with a conductivity ranges from $3.2 \times 10^{-10} \mathrm{~m} / \mathrm{s}$ to $1 \times 10^{-13} \mathrm{~m} / \mathrm{s}$, and with a porosity ranges from $0.01-0.005$.

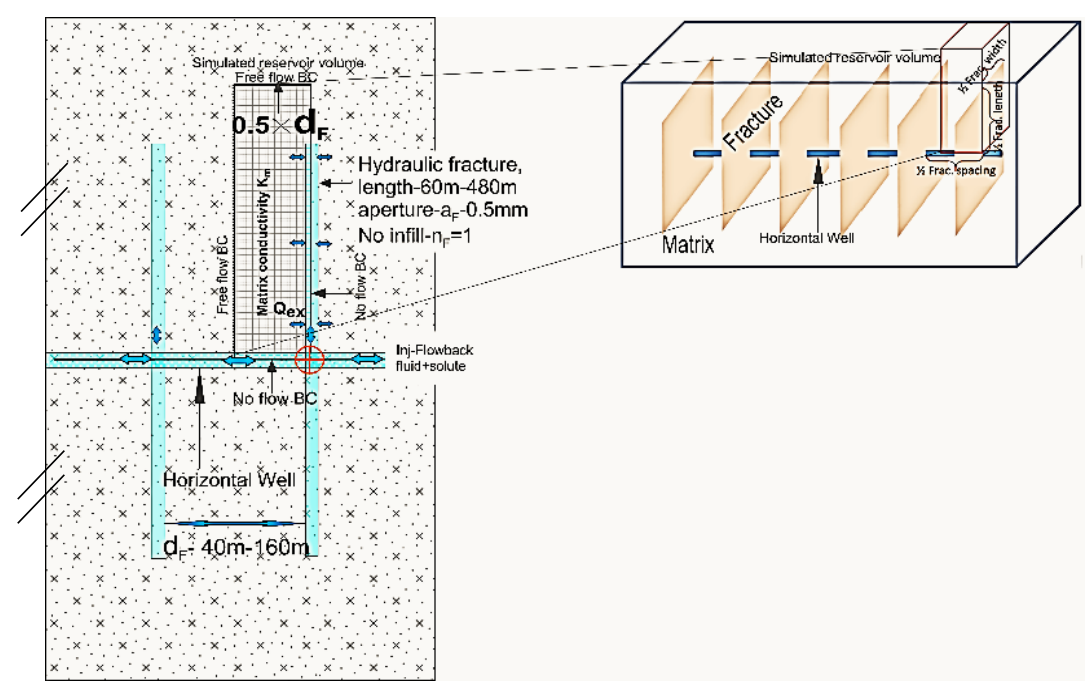

Figure 2.3.1: Conceptual model of HDR type EGS formation and injection- flowback well. The red box indicates fracture and matrix domain for simulation that pertaining 1/8 of a fracture volume from equally spaced fractures.

\section{Results}

A range of MSTs flowback signal is simulated using different tracer injection duration as well as injection volume in HDR type parallel fracture stimulated geo-reservoirs. Conservative tracer, as well as sorptive tracer flowback signals for various fracture length in fracture aperture of $2 \mathrm{~mm}$ and $1 \mathrm{~mm}$ geo-reservoirs, is considered for the simulation. Two classes MSTs (Karmakar et al. 2015a and 2015b) is sorted viz. weak MSTs and strong MSTs based on relative strength of sorption coefficient on fracture wall (matrix sorption). Fracture length $60 \mathrm{~m}, 120 \mathrm{~m}$, $180 \mathrm{~m}, 210,240 \mathrm{~m} 420 \mathrm{~m}$ and $480 \mathrm{~m}$ are considered for weak matrix sorptive tracer $(\mathrm{k}-0.01,0.1$, $1,2)$ and strongly matrix sorption tracer $(5,10,30,50,100)$ with a matrix porosity of $1 \%$ and $0.5 \%$. The early flowback tracer signal is sensitive to a certain fracture length range (Fig 2.3.2). Fig. 2.3.3 reveal that low/weak sorptive tracer is relatively sensitive to the fracture length. It must me noted that, while very low fracture length is assumed from a stimulated fracture in HDR formation, using a very low sorptive tracer for a specific chaser volume, it would act as a conservative tracer and reveal the whole fracture length and flow into the matrix. In that instance, it may reveal a cyclic form of tracer curve from different fracture length which means 
additional ambiguity with the tracer signal, and the clear cut relationship with fracture length and tracer flowback signal will be broken. It follows the monotonic trend of lower breakthrough concentration with lower fracture length.

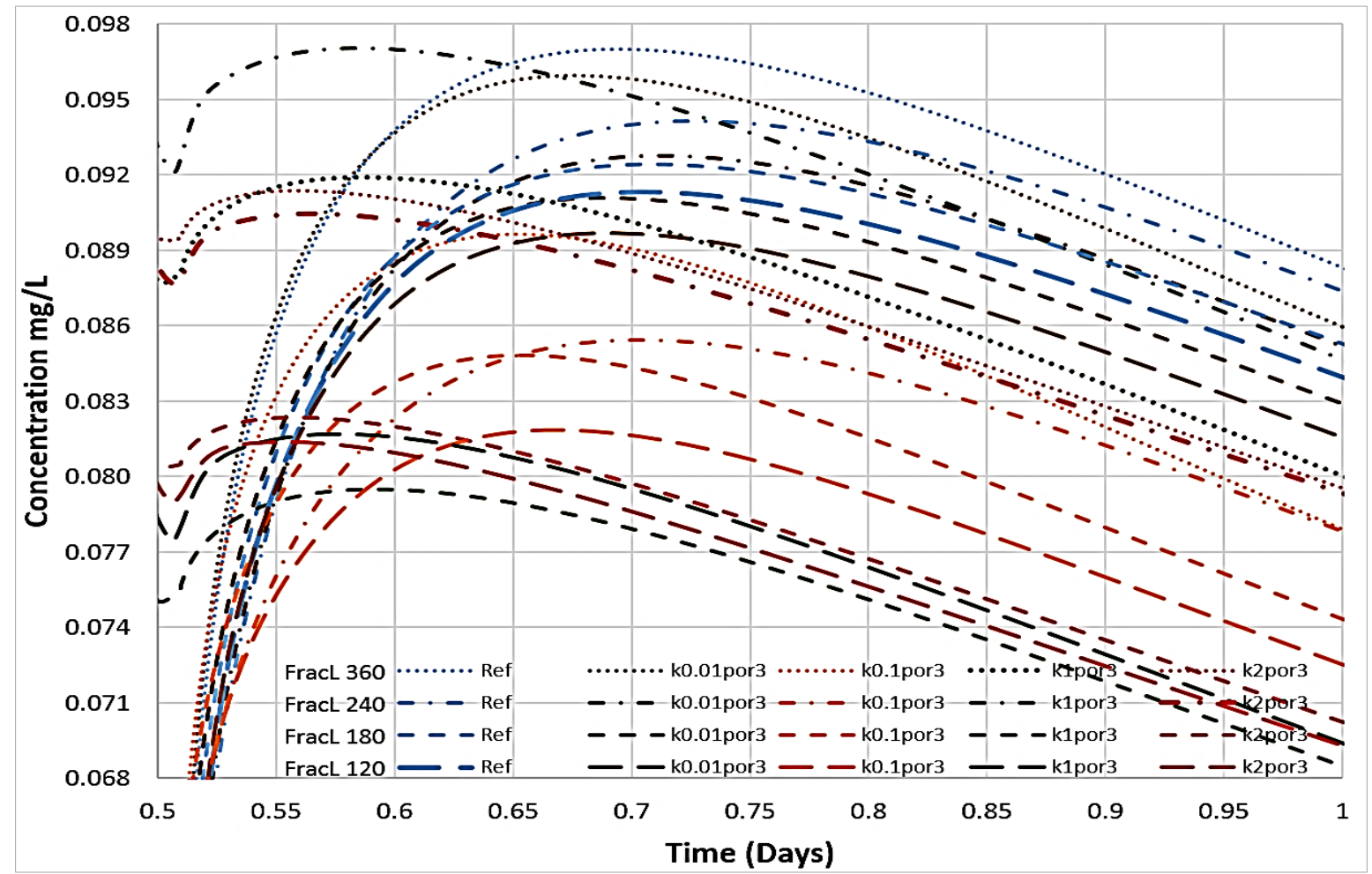

Figure 2.3.2: Weak matrix sorption tracer (MSTs) sensitivity in different fracture length for hydraulically stimulated fracture in HDR type EGS. It shows that very weak sorptive or conservative tracer $R=1-1.5$, matrix porosity $3 \%, k=0-0.01$ is sensitive to the fracture length while a big aperture $(1 \mathrm{~mm}-2 \mathrm{~mm})$ is created. 


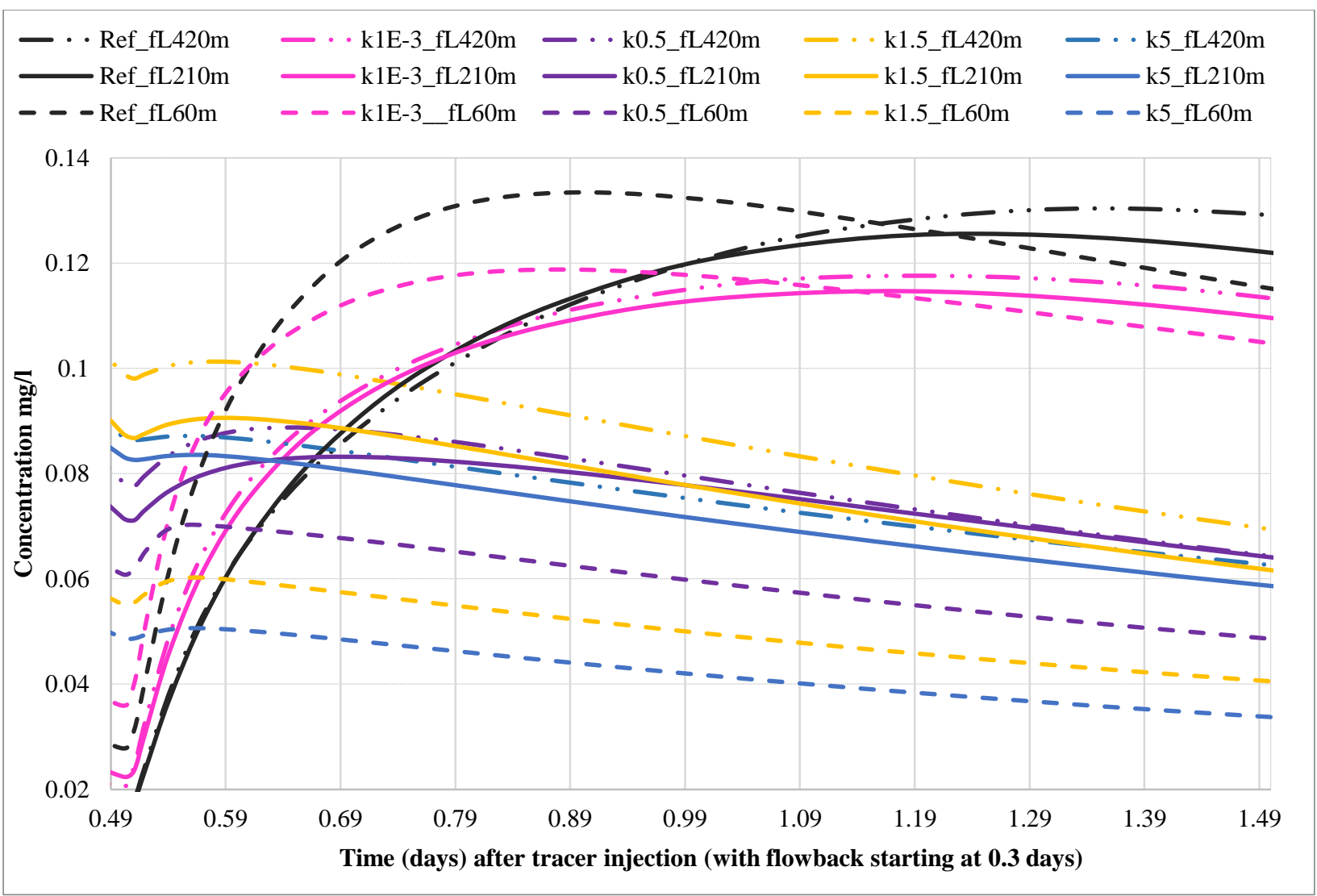

Figure 2.3.3: Strong MST (matrix sorption tracer) sensitivity in different fracture length for hydraulically stimulated fracture in HDR type EGS, matrix porosity $1 \%$ for a fracture aperture $2 \mathrm{~mm}$. Sensitive sorptive tracer range is $k-0.5-1.5$. 


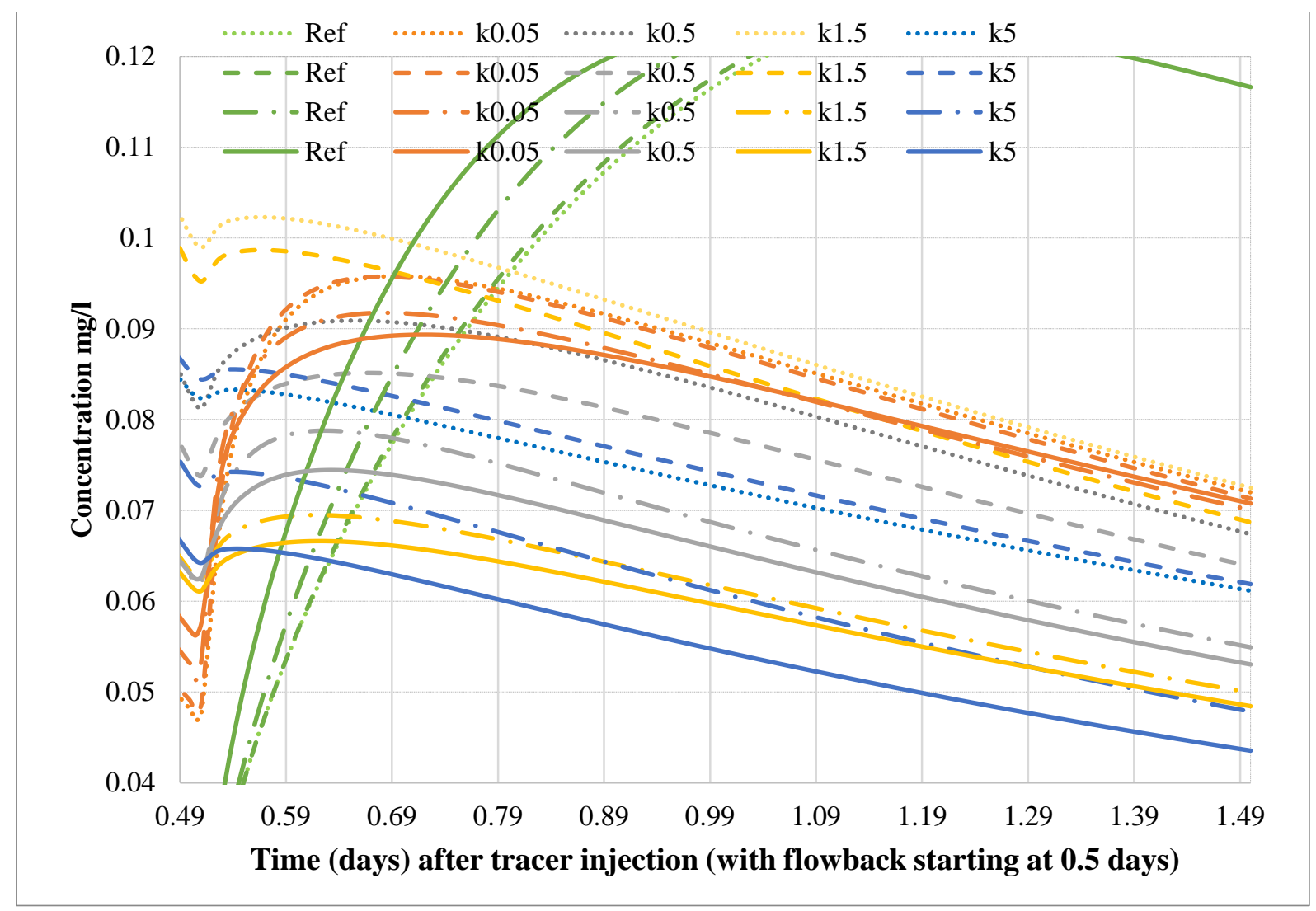

Figure 2.3.4: Medium range MSTs sensitivity in different fracture length for hydraulically stimulated fracture in HDR type EGS, matrix porosity $1 \%$ for a fracture aperture $1 \mathrm{~mm}$. Sensitive sorptive tracer range is $k-0.5-1.5$.

Figures 2.3.3 and 2.3.4 shows that strong sorptive tracer (here $\mathrm{k}=1.5$ to 5 ), is sensitive to the fracture length up to $210 \mathrm{~m}$, and it shows a very narrow range of tracer concentration for lower fracture length. And a low sorptive or conservative tracer signal is independent of fracture length. However, with higher sorptivity of solute tracer, tracer signal sensitivity is not clearly understood due very low penetration length and less number of mesh element to interpolate or calculate tracer concentration near to the test well. We observed solute tracers pull signals were independent or very low sensitive to matrix porosity (Fig. 2.3.2) as well as matrix permeability due to very high contrast in flow regime between fracture and matrix. A medium sorptive tracer range is found sensitive for a broad range of fracture length $120 \mathrm{~m}$ to $480 \mathrm{~m}$ in the case of fracture aperture $1 \mathrm{~mm}-2 \mathrm{~mm}$. Hence using a particular range of matrix sorptive tracer will be effective to estimate fracture length (Figs. 2.3.3, 2.3.4). 


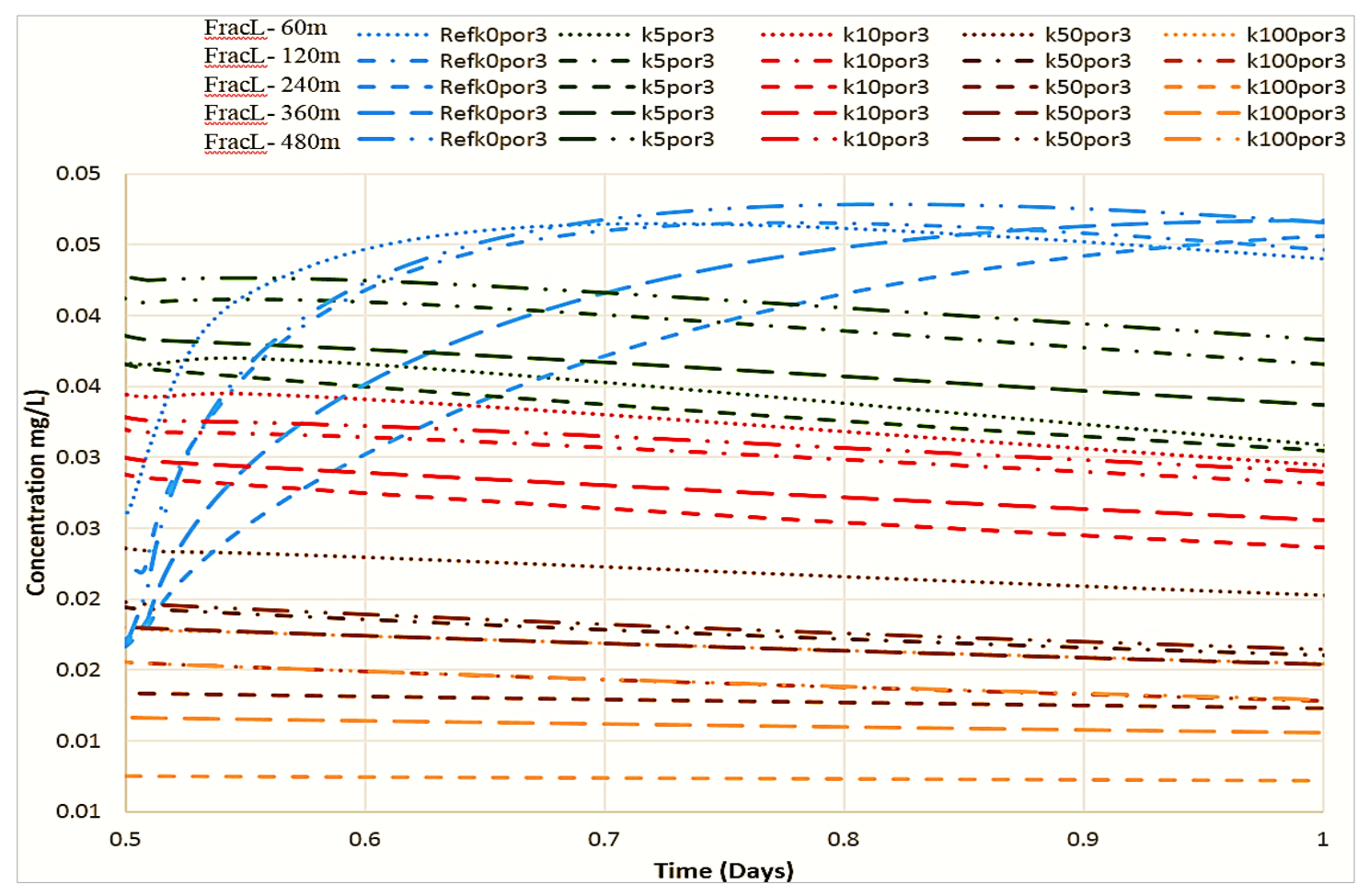

Figure 2.3.5: Effect of higher injection rate/volume, which exceed the fracture volume, for the high MST (k 5-100, matrix porosity-3\%). It shows that strong MSTs show no clear trend with the fracture length.

From the tracer breakthrough, it revealed that fracture length influence tracer breakthrough for the higher matrix sorptive tracer. To have a consistent tracer signal one should choose very high sorption tracer or very lower sorption tracer while fracture length is the parameter in question. However, it is important to note that fracture aperture must know before estimating the extent of fracture length from this kind of test. The monotonicity of the tracer signal 'cyclically reversed' while injection volume is higher than fracture volume (Figs. 2.3.5 and 2.3.6). Hence, during parameter inversion, it needs to be cautious and necessary to consider the injection volume design to avoid ambiguous fracture length estimation.

\section{Sorption tracer selection and sensitivity}

Sorptive tracer is particularly useful to determine fracture size in stimulated reservoir both in sedimentary and crystalline formations which are the particular interest in method development process in earlier parts (c.f. part 1 and 2) of this chapter. However, it is important to make available a range of sorption properties would be applied for this kind of test. In that instance, it observes a decreased sensitivity for fracture thickness with an increase of sorption properties in case of proppant sorptive tracer in part 1 and part 2. From above sorptive tracer implementation case in HDR type EGS, it also perceives not a universal range of sorptive tracer would be effective nor all range sensitive for different stimulated georeservoir formation. In 
that instance, a medium to higher range sorptive tracer would be particular importance for HDR application for fracture length as it would not be affected by lower fracture volume. However, for fracture length estimation should follow after a preliminary evaluation which would determine the appropriate range of sorption tracer to be used.

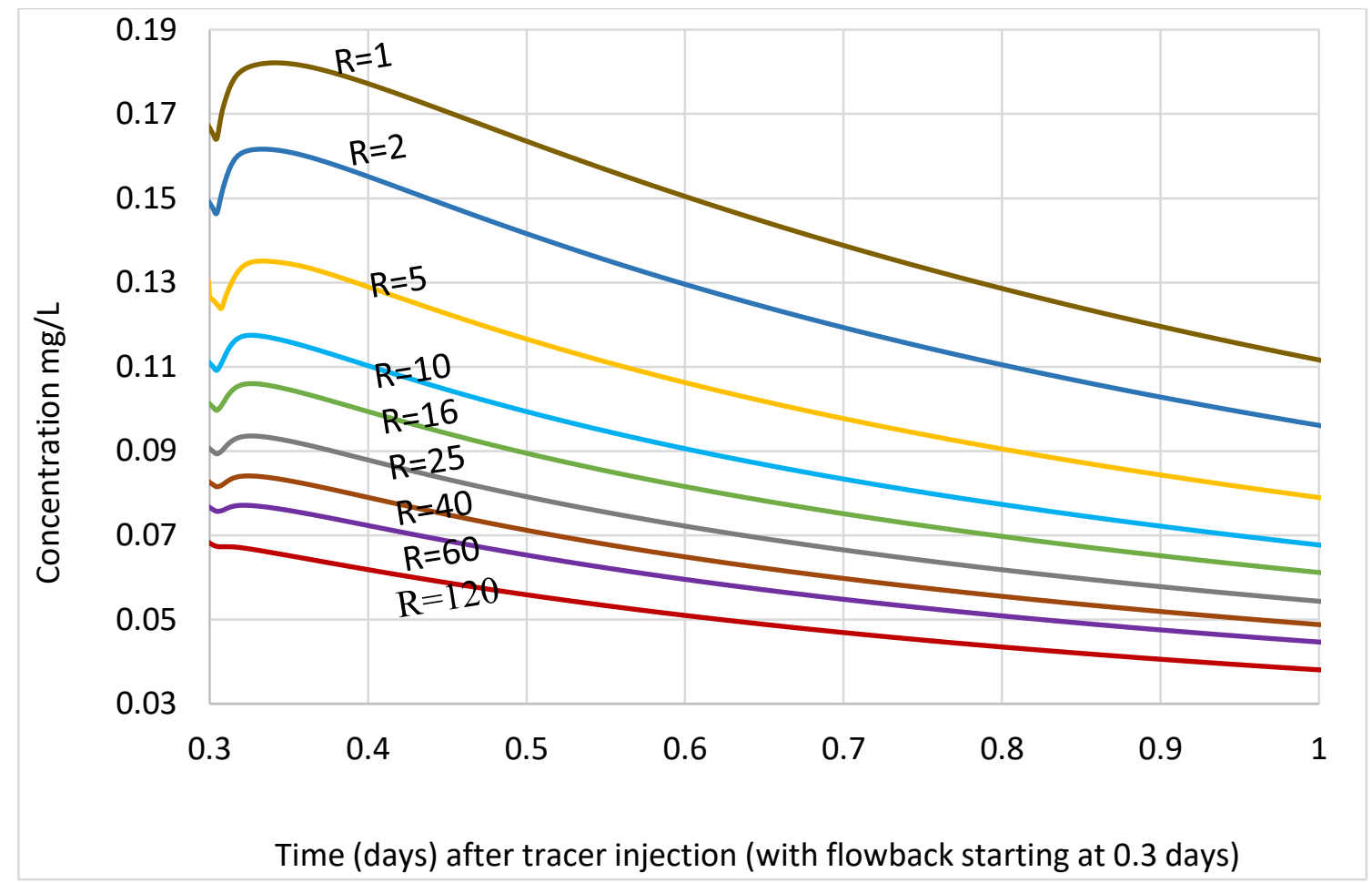

Figure 2.3.6: Simulated signals of multiple PST and MST (characterized by different Kd values) during GPF flowback, at a fixed value of GPF thickness $(12 \mathrm{~mm})$ and proppant-packing porosity (30\%). 


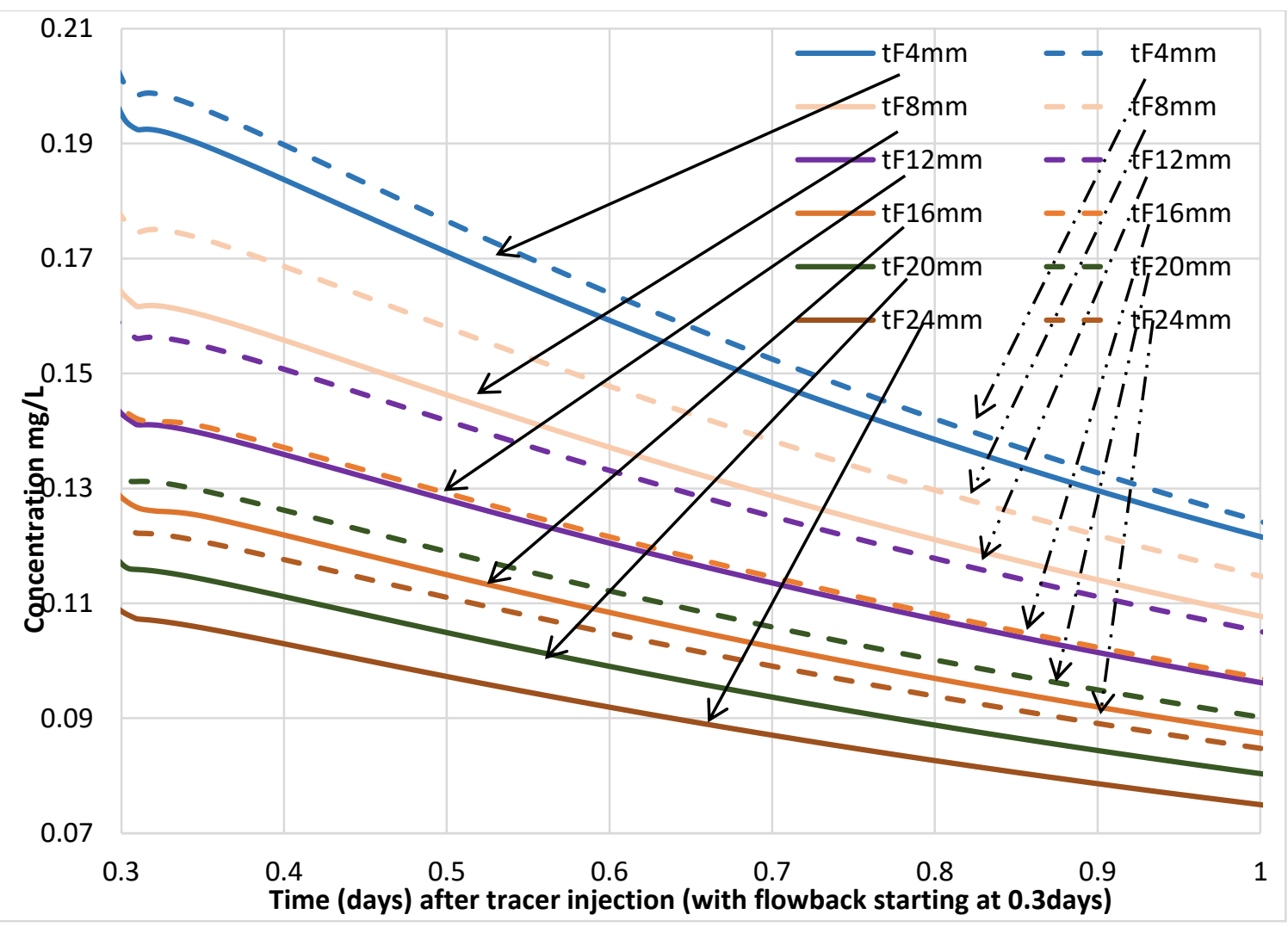

Figure 2.3.7: Simulated signals of multiple PST during GPF flowback, at a different value of GPF and proppant-packing porosity (30\% and 60\%).

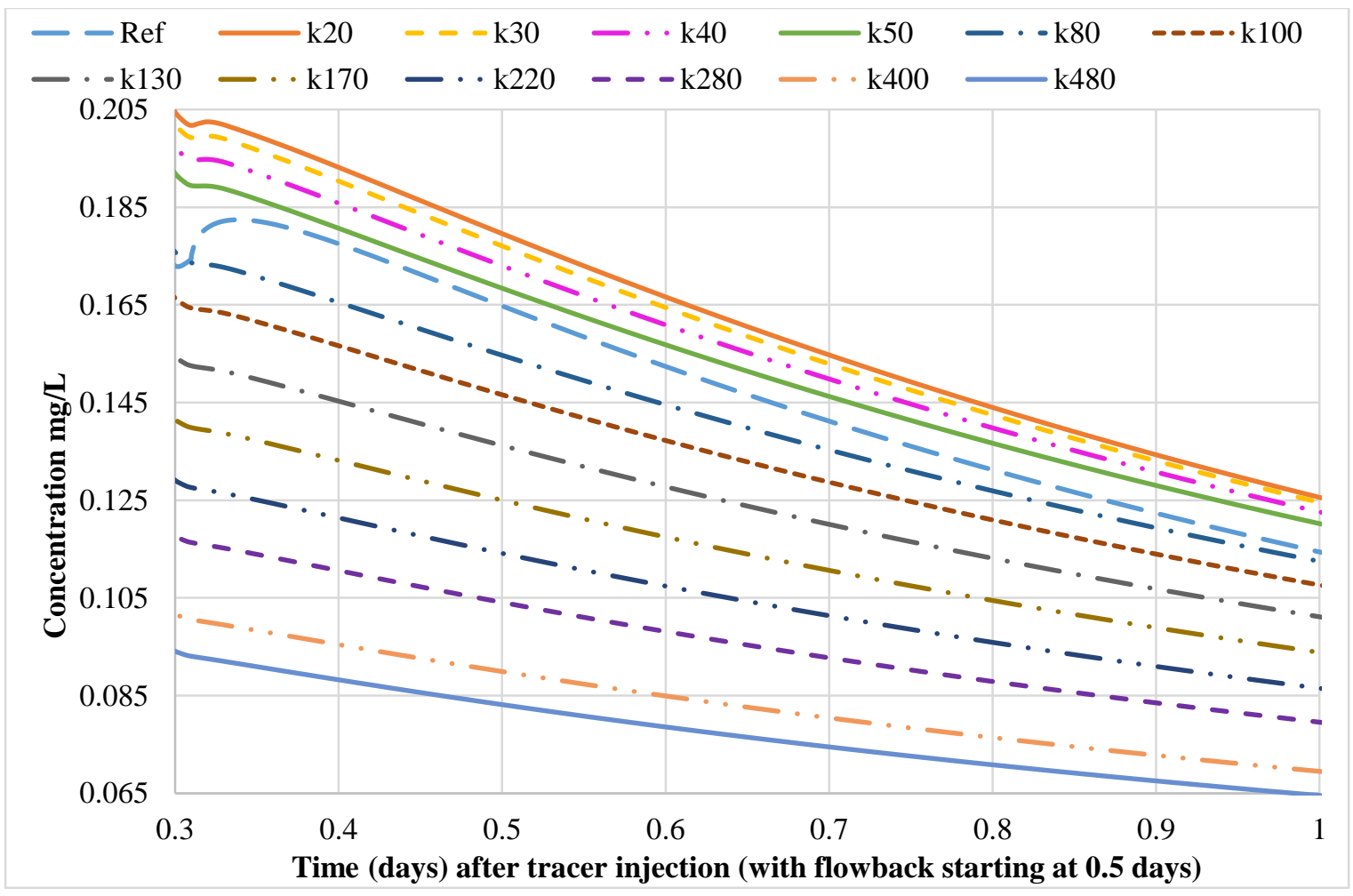


Figure 2.3.8: Simulated signals of multiple PST (characterized by different Kd values) during GPF flowback, at a fixed value of GPF thickness $(12 \mathrm{~mm})$ and proppant-packing porosity (40\%). Signals are labeled by the retardation factor $k$ ( $k=K d \times$ proppant density).

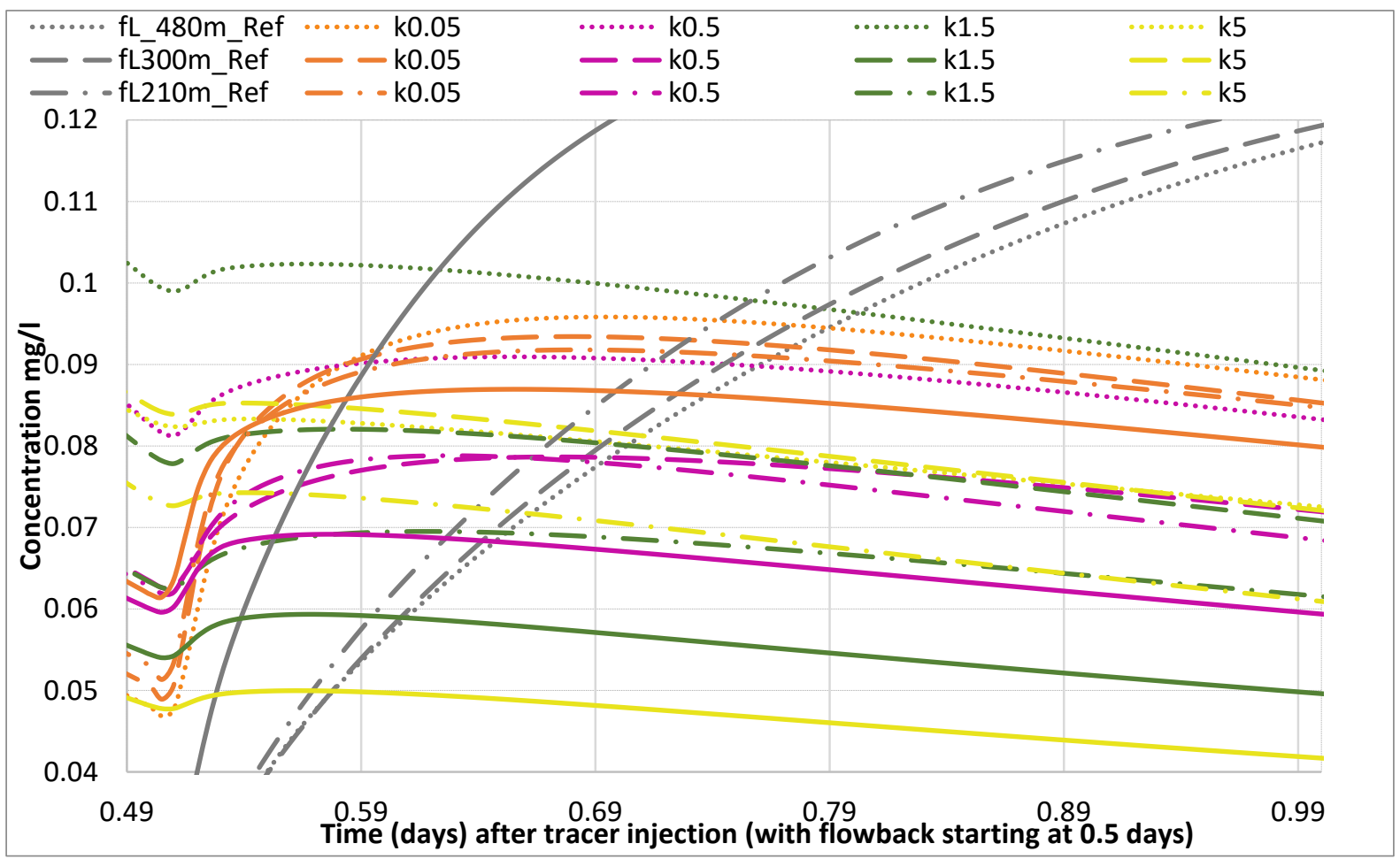

Figure 2.3.9: The injection volume is in the same size or higher that fracture volume for lower two example fracture length, which break the monotonicity of tracer breakthrough and signal grows rather reversibly.

\section{Discussion and conclusion}

The minimum sorptivity (minimum $\kappa$ value) that required to induce sufficient contrast between measurable tracer signals for different values of target parameters has discussed in chapeter 2 part 1 (see also in Karmakar et al 2015a). The scheme is: a $p \%$ change in a target parameter value produces $c \%$ change in the signal of a conservative tracer, and a $s \%$ change in the signal of a sorptive tracer, $s$ being a function of $\kappa$. For PSTs and MSTs based SWIF test study, a lower sorptive tracer than its minimum necessary is suggested, assuming an increase sensitivity factor (ratio between sorptive tracer signal changes to conservative tracer signals changes, s/c) approximately equal to $\sqrt{ }(1+0.7 \times$ sorption coefficient, $\kappa)$. This empirical relationship delimits that too much investment on highly sorptive tracer will not affect tracer parameter sensitivity at same magnitude (Figure 2.3.4 and 2.3.5). One needs to note that the higher the tracer's retardation, the lower is its fracture invasion, and consequently a poorer capability for characterizing the fracture as a whole. In principle, this could be compensated by increasing 
the chaser volume (i.e., by injecting sorptive tracers earlier than conservative tracers) or longer injection duration rather applying higher the injection rate. Hence, for early-time tracer injection-flowback test, it is advisable to apply a medium ranged MSTs $(0.7<\mathrm{k}<2.5$, matrix porosity $3 \%)$ and PSTs $(25<\mathrm{k}<80)$, to avoid ambiguity due to the error in detection.

Table 2: Solute tracer uses and sensitivity with different EGS georeservoirs

\begin{tabular}{|c|c|c|c|}
\hline $\begin{array}{l}\text { Stimulation } \\
\text { techniques }\end{array}$ & $\begin{array}{l}\text { Gel proppant } \\
\text { fracture (GPF) }\end{array}$ & \multicolumn{2}{|l|}{$\begin{array}{l}\text { Water fracture } \\
\text { (WF) }\end{array}$} \\
\hline EGS type & $\begin{array}{l}\text { Sedimentary } \\
\text { formation }\end{array}$ & Single fracture & $\begin{array}{l}\text { Parallel fracture in } \\
\text { HDR type }\end{array}$ \\
\hline $\begin{array}{l}\text { Sensitive } \\
\text { parameters }\end{array}$ & $\begin{array}{l}\text { Fracture } \\
\text { thickness and } \\
\text { fracture porosity }\end{array}$ & $\begin{array}{l}\text { Longitudinal } \\
\text { dispersivity, Fracture } \\
\text { aperture }\end{array}$ & Fracture length \\
\hline Tracer types & PSTs and MSTs & Conservative tracers & MSTs \\
\hline $\begin{array}{l}\text { Tracer ranges } \\
\mathrm{R}\end{array}$ & $\begin{array}{l}\text { MSTs-k=0.7-2.5, } \\
\text { Matrix porosity } \\
\varphi_{\mathrm{m}}=3 \% \\
\text { PSTs- } 25<\mathrm{k}<80\end{array}$ & $\begin{array}{l}\text { Effective with } \\
\text { Fracture aperture- } \\
1.08 \mathrm{~mm}-0.18 \mathrm{~mm}\end{array}$ & $\begin{array}{l}\text { MSTs, } \mathrm{k}=0.5-1.5 \\
\varphi_{\mathrm{m}}=1 \% \\
\text { Fracture aperture } 0.5 \\
\mathrm{~mm}-2 \mathrm{~mm}\end{array}$ \\
\hline $\begin{array}{l}\text { Injection } \\
\text { Volume/Tpush }\end{array}$ & monotonic & monotonic & $\begin{array}{l}\text { Affect sensitivity, } \\
\text { Variable injection } \\
\text { volume would be } \\
\text { effective }\end{array}$ \\
\hline
\end{tabular}

Figures 2.3.2 and 2.3.6 suggest that for the fracture length estimation from matrix sorptive tracer, it is essential to evaluate the tracer signal at very early stage. The 'early-middle' or 'early-late time' tracer signals would be insensitive to the fracture length for low sorptive tracer $\left(\mathrm{k}-0.7 \varphi_{\mathrm{m}}=1 \%\right)$ as well as high sorption tracer $\left(\mathrm{k}-1.5 \varphi_{\mathrm{m}}=1 \%\right)$ as it observed that the stronger the sorption related retardation, the earlier the 'late time regime' (Ghergut et al., 2013b). This is because the stronger the retardation, the shorter the pervasion distance into the fracture and thus the corresponding 'back travel' time. Moreover, in all the cases of fracture length, tracer comprehends a tiny part of the matrix and has little penetration too due to very short tracer injection duration and small injection volume too.

From the simulation results, in HDR type EGS geo-reservoir, tracer application scheme is recommended to evaluate fracture length as follows- a) Weak matrix sorptive tracers for relatively longer fracture length/higher fracture volume. The matrix sorption coefficient $\kappa$ value should be a range of 0.1 to 1 for a matrix porosity $0.5 \%-3 \%$. b) Strong matrix sorptive tracer would be recommended for all cases however specifically useful while stimulated fracture length expected to be relatively small $(<200 \mathrm{~m})$. c) Early-time tracer signal from conservative 
solute can determine the aperture of fractures created by a WF treatment. However, the effectiveness needs to be verified for very thin fractures $(<0.2 \mathrm{~mm})$ as suggested in (Karmakar et al., 2015a and 2015b).

Acknowledgments: The first author gratefully acknowledges continued financial support from the DAAD (German Academic Exchange Service) to pursue his Ph.D. work. This work also benefited from long-term financial and technical support granted by the Federal German Ministry for Economic Affairs and Energy (BMWi) within the project TRENDS (2014-2018, FKZ 0325515).

\section{References}

Breede K, Dzebisashvili K, Liu X, Falcone G, 2013, A Systematic review of enhanced (or engineered) geothermal systems: past, present and future. Geothermal Energy. doi:10.1186/2195-9706-1-4.

Diersch, H.-J., G., 2009. FEFLOW 5.4: finite element subsurface flow and transport simulation system. Reference manual. DHI-WASY Ltd., Berlin, Germany, 292 pp

Jiang F., Chen, Huang, W. Luo L. 2014, A three-dimensional transient model for EGS subsurface thermo-hydraulic process, Energy 72, 300-310.

Jung R., 2013, EGS - Goodbye or Back to the Future 95, Effective and Sustainable Hydraulic Fracturing, Dr. Rob Jeffrey (Ed.), ISBN: 978-953-51-1137-5,InTech, DOI: 10.5772/56458. Available from: http://www.intechopen.com/books/effective-and-sustainable-hydraulicfracturing/egs-goodbye-or-back-to-the-future-95.

Ghergut, I., Behrens, H., Sauter, M., 2013a. Can Peclet numbers depend on tracer species? going beyond SW test insensitivity to advection or equilibrium exchange. In: Proceedings 38th Workshop on Geothermal Reservoir Engineering, Stanford Univ. (CA), SGP-TR-198, 326335 .

Ghergut, I., Behrens, H., Sauter, M., 2013b. Single-well tracer push-pull test sensitivity to fracture aperture and spacing. In: Proceedings 38th Workshop on Geothermal Reservoir Engineering, Stanford Univ. (CA), SGP-TR-198, 295-308.

Karmakar, S., Ghergut, I., Sauter, M., 2015a, EGS in sedimentary basins: sensitivity of earlyflowback tracer signals to induced-fracture parameters. Energy Procedia 76, 223-229.

Karmakar, S., Ghergut, J., Sauter, M., 2015b, Early-flowback tracer signals for fracture characterization in an EGS developed in deep crystalline and sedimentary formations: a parametric study, Geothermics, in press, doi:10.1016/j.geothermics.2015.08.007

Schulte T, Zimmermann G, Vuataz F, Portier S, Tischner T, Junker R, Jatho R, Huenges E 2010, Enhancing geothermal reservoirs. In: Huenges E (ed) Geothermal Energy Systems: Exploration, Development, and Utilization. Wiley-VCH, Berlin, pp 173-243 
CHAPTER 3-PART 1: Single-well tracer injection-flowback test simulation in fractured georeservoir using finite element code FEFLOW and COMSOL- a benchmark study

Shyamal Karmakar, Sandra Oehlmann, Julia Ghergut, Martin Sauter

Citation:

Karmakar, S., Oehlmann, S., Ghergut, J., Sauter, M., Benchmark study on flow and solute transport in geological reservoirs, draft paper

Geoscience Centre of the University of Göttingen, Department of Applied Geology,

Goldschmidtstrasse 3, 37077 Göttingen, Germany

*Corresponding author: shyamal.karmakar@geo.uni-goettingen.de 


\section{Abstract}

The single well push-pull tracer simulation study in chapter 2 of this thesis uses a single fracture model with tetrahedral mesh. The flow is assumed as cubic law for 'no' infill fracture (waterfracture) and Darcy flow for gel-proppant filled fracture in the numerical modeling package FEFLOW. The simulation results were reproduced in this part thesis using higher temporal and spatial discretization in FEFLOW. At the same time, the same model was set in COMSOL Multiphysics, which make uses of fracture and matrix model as a hybrid model of 3D matrix domain with a lower dimention-2D fracture element. The result was compared qualitatively in terms of capturing the spikes of injection and flowback in early flow back tracer test. From this two approaches of simulation, it is revealed that at early times, the numerical dispersion is an issue for conservative tracer simulation, while it is negligible for sorptive tracers. Moreover, COMSOLs PDE interface is a useful tool to build and simulate flow and solute transport for a fracture domain as well.

\section{Model concept}

This study compares the simulation results of two finite element based modeling software packages viz. FEFLOW and COMSOL Multiphysics for flow and solute transport for a single well tracer push-pull test in a single fracture model. The difference between the simulation results and the computational efficiency between the two numerical simulators are quantified for single-well tracer push-pull test for multi-tracer use in conjunction with gel-proppant fracture (GPF) treatments. The numerical model in FEFLOW and COMSOL consider two simplified fracture models based on lithostratigraphic and hydrogeologic data from the Geothermal In-Situ Laboratory at Groß-Schönebeck in the NE-German basin (c.f. Karmakar et al 2015a, 2015b). The commercially available finite-element software FEFLOW 5.4 (Diersch, 2009) and COMSOL Multiphysics 4.4 were used to simulate fluid flow and tracer transport in fractures resulting from GPF, relying on some simplifying assumptions. The rock matrix is treated like a single-porosity medium in $3 \mathrm{D}$, whereas vertical planar fractures are represented by lower-dimensional (2D) so-called 'discrete elements'. Flow and transport in fracture and matrix compartments are coupled to each other by FEFLOW's special, computationally efficient approach. Flow within water-fracture type (WF) fractures is assumed to obey the 'cubic law' (Tsang, 1992); flow within the matrix, as well as within GPF-type fractures, is assumed as Darcian. Thus, GPF-type fractures are treated almost like an ordinary porous medium, which (with the exception of solute exchange with the adjacent rock matrix) 
is similar to the 'push-pull' test situation considered by Schroth et al. (2001). Solute transport in fractures, and within rock matrix is assumed as advective-dispersive, with a number of additional processes at fluid-solid interfaces as described below (adsorption-desorption onto/from matrix rock, or proppant coatings; matrix diffusion). The model has to be 3D because of its fracture (approximately radial) and matrix (approximately linear) flow and transport components. The assumption of horizontal and vertical symmetry and neglecting gravity effects means that it needs to consider only one-quarter of the fracture plane, one-half of the fracture aperture or thickness and one-eighth of its adjacent matrix block (similar to the model used by Ghergut et al., 2013b for parallel-fracture systems). The geometry of model compartments (fracture and matrix block) and the hydrogeological parameter distribution for the WF and GPF target layers are shown in Fig. 3.1.1. The assumption of isotropic permeability (described by a single scalar value for each compartment) is rather unrealistic, but it does not influence the parameter sensitivity behaviour of simulated tracer signals. Further, we assume isothermal conditions; the hydraulic conductivity values listed in Fig. 3.1.1 corresponds to a temperature of $120^{\circ} \mathrm{C}$, assumed to prevail during tracer injection and early flowback, as a transient 'equilibrium' between native reservoir conditions $\left(\sim 160^{\circ} \mathrm{C}\right)$ and injected fluid temperature $\left(\sim 15^{\circ} \mathrm{C}\right)$. 


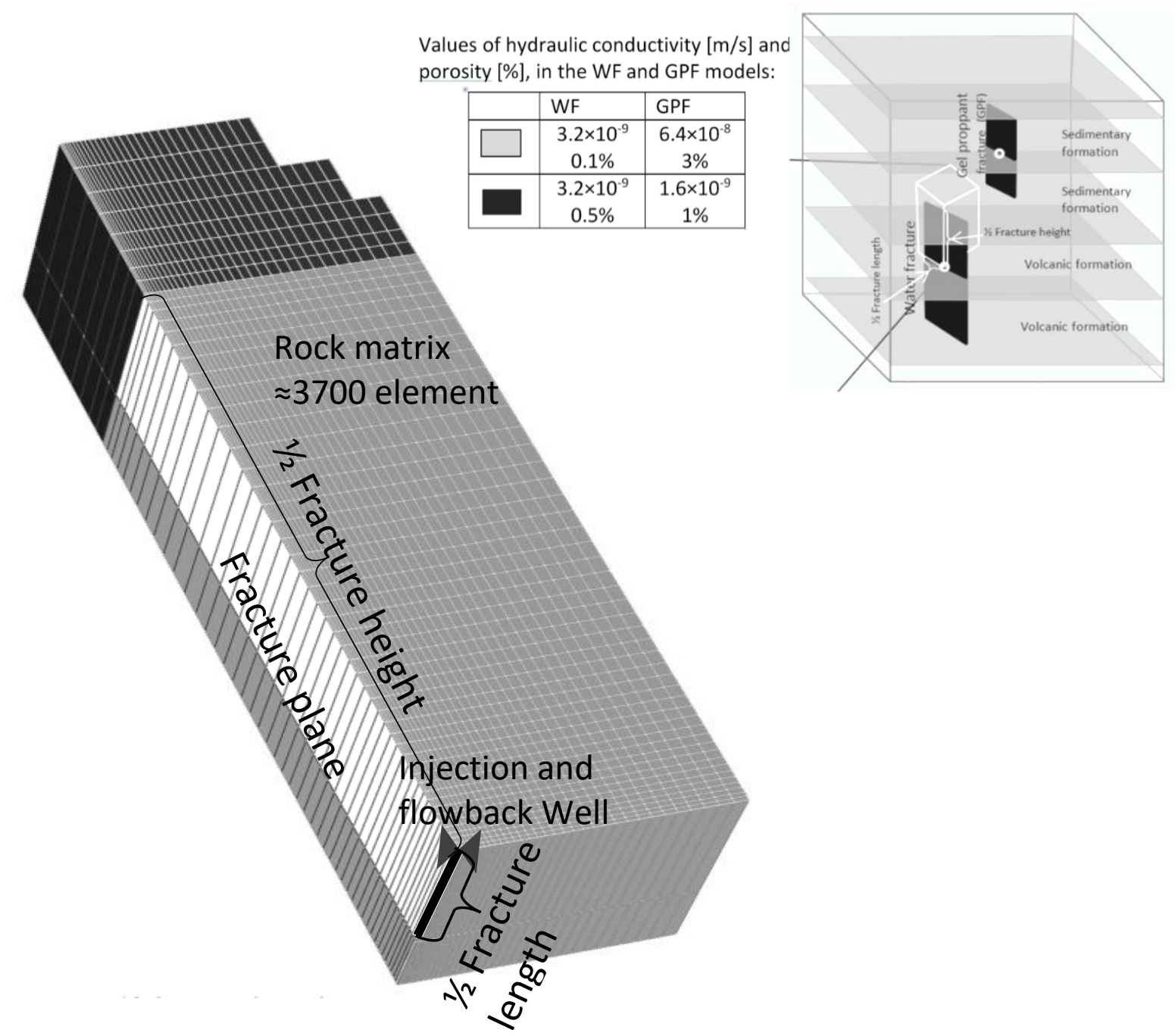

Figure 3.1.1: 3D model domain and hydrogeological parameter distribution. The rectangular mesh shown here is used in FEFLOW simulation. COMSOL simulation is done in the triangular mesh.

Flow and solute transport model PDE describe for 2D, phreatic, Darcy flow in Diersch (2009) used in FEFLOW as follows.

$$
S \frac{d h}{d x}-\nabla \cdot(K B \cdot(\nabla h+\theta e))-Q=0
$$

In water fracture flow, $\mathrm{K}$ evaluated as shown below

$$
\text { Hagen Poisuillae (Cubic law), } K=\frac{b^{3} \rho_{0} g}{12 \mu_{0}}
$$

Here $\mathrm{S}\left[\mathrm{L}^{-1}\right]$ - specific storage, $\mathrm{K}\left[\mathrm{LT}^{-1}\right]$ - Darcy conductivity in fracture or Conductivity as cubic law fracture , b [L] is the total fracture thickness for gel-proppant fracture or fracture hydraulic aperture for water-fracture (Tang et al 1992), dynamic viscosity $\mu_{0}\left[\mathrm{MLT}^{3}\right]$, density of fluid, $\rho_{0}\left[\mathrm{ML}^{-3}\right]$, source or sink of fluid $\mathrm{Q}\left[\mathrm{L}^{3} \mathrm{~T}^{-1}\right]$. 
Solute transport in fracture

$$
\begin{gathered}
S \frac{d c}{d t}+q \cdot \nabla c-\nabla \cdot(B \varepsilon D \cdot \nabla C)+B\left(\varepsilon Q_{p}+\vartheta\right)-Q_{c}=0 \\
\text { Dispersivity, } D=D_{d}+D_{m}
\end{gathered}
$$

$\mathrm{q}\left[L T^{-1}\right]$ - Darcy velocity vector, $\vartheta$-degradation or decay, $D_{d}\left[L T^{-2}\right]$-hydrodynamic dispersivity in fracture, $D_{m}\left[\mathrm{~L}^{2} \mathrm{~T}^{-1}\right]$ - molecular diffusion of solutes

Hydrodynamic dispersion tensor used in FEFLOW according to Bear-Scheidegger dispersion tensor for matrix and fracture

$$
\begin{gathered}
D_{\text {matrix }}=D_{d}+D_{m}=D_{d}+\beta_{T} v+\left(\beta_{L}-\beta_{T}\right) \frac{q \otimes q}{|q|} \ldots \ldots \ldots . . . \\
D_{\text {fracture }}=D_{d}+D_{m}=D_{d}+\beta_{L} \frac{q \otimes q}{|q|} \ldots \ldots \ldots \text { (5) }
\end{gathered}
$$

Whereas, the dispersion tensor in COMSOL implemented as Burnett and Frind (1987) read as

$$
D_{\text {fracture }}=D_{d}+D_{m}=D_{d}+\left(\beta_{L}-\beta_{T}\right) \frac{q \otimes q}{|q|}
$$

A derivative of retardation coefficient, $\mathrm{R}$ also used for the background solute retardation which is not relevant for this study as initial concentration $\mathrm{C}_{0}$ assumed both in fracture and matrix and $\mathrm{R}$ is defined as

$$
R=1+\rho \times K_{d} \times \frac{1-n}{n}=1+\kappa \times \frac{1-\mathrm{n}}{\mathrm{n}}
$$

Where, as $\kappa=\rho \times K_{d}, \kappa$ is the sorption coefficient, $K_{d}$ []-sorption distribution coefficient (slope of linear segment of adsorption isotherm, Freeze and Cherry, 1979), $n$ [ ] -is the porosity medium (for matrix sorptive tracer, matrix porosity and for proppant sorptive tracer, proppant packing), $\rho\left[M L^{-3}\right]$ - intrinsic the density of solid-phase material (either rock or proppants).

The retardation factor $R$ is not an intrinsic tracer property, but it also depends on the porosity of the transport medium; as equation 7 shows $R$-values cannot be varied independently of porosity.

The exchange between fracture and matrix implemented in COMSOL as 


$$
\begin{aligned}
& Q_{\text {matrix-frac }}=\frac{1}{t F} \times\left(D_{\text {mol }} \times\left(C_{f}-C_{m}\right)+\frac{e x(P)}{\rho_{f}} \times C_{f}(\text { if ex }(P) \geq 0)+\frac{e x(P)}{\rho_{f}} \times C_{m}(\text { if ex }(P)<0)\right. \\
& Q_{\text {frac-matrix }}=\frac{1}{t F} \times\left(D_{\text {mol }} \times\left(C_{m}-C_{f}\right)-\frac{e x(P)}{\rho_{f}} \times C_{m}(\text { if ex }(P) \leq 0)+\frac{\operatorname{ex}(P)}{\rho_{f}} \times C_{f}(\text { if ex }(P)>0)\right.
\end{aligned}
$$

Here $t F[L]$ is the fracture total thickness (Tsang 1992), $C_{f / m}\left[M L^{-1}\right]$-concentraiton in fracture (f) or matrix (m), ex $(P)\left[M L^{-1} T^{-2}\right]$ - is the pressure gradient at the exchange surface between fracture plane and matrix, $\rho_{f}\left[M L^{-3}\right]$-fluid density, $D_{m o l}\left[L^{2} T^{-1}\right]$-matrix diffusion

Using COMSOLs coefficient form PDE interface, solute transport in the fracture is formulated as shown in equation (3). The exchange between fracture and matrix (as shown in equation 7 and 8) implemented following matrix-fracture exchange term (Oehlmann et al 2015) for gelproppant filled fracture as well as water-fracture. The exchange term indicated as source flux, determines the flow (calculated from pressure gradient) and solute transport (equation 7 and 8 ) between fracture and adjacent matrix. COMSOL 4.4 allows no local refinement for rectangular meshes. Therefore, to facilitate local refinement flexibility for push-pull a well and adjacent region, a triangular mesh is used in COMSOL, whereas a rectangular mesh which is pressed near the fracture plane and injection point (Fig. 3.1.1) is used in FEFLOW. COMSOLs subsurface flow module, includes a 'Darcy' flow based fracture flow module which is also possible to edit to 'cubic law' flow by including the respective equation for the hydraulic conductivity K. However, Darcy flow formulation is used for gel-proppant type fracture where the fracture volume is packed with proppant that is analogous to the porosity of porous media. This study considers two cases of porosity or proppant packing viz. 50\% and 35\% for two total fracture thickness- $12 \mathrm{~mm}$ and $20 \mathrm{~mm}$.

\section{Results and Discussion}

\subsection{Spatial and Temporal discretization effect on FEFLOW single fracture SW injection} flowback tracer breakthrough

From figure 3.1-2, it is revealed that with an increase in refinement can accentuate the tracer breakthrough efficiency or precision. However, the computation cost is significantly higher for the amount of precision, which is more or less not a necessary goal of this simulation towards tracer test design or parameter inversion from field tracer test. 


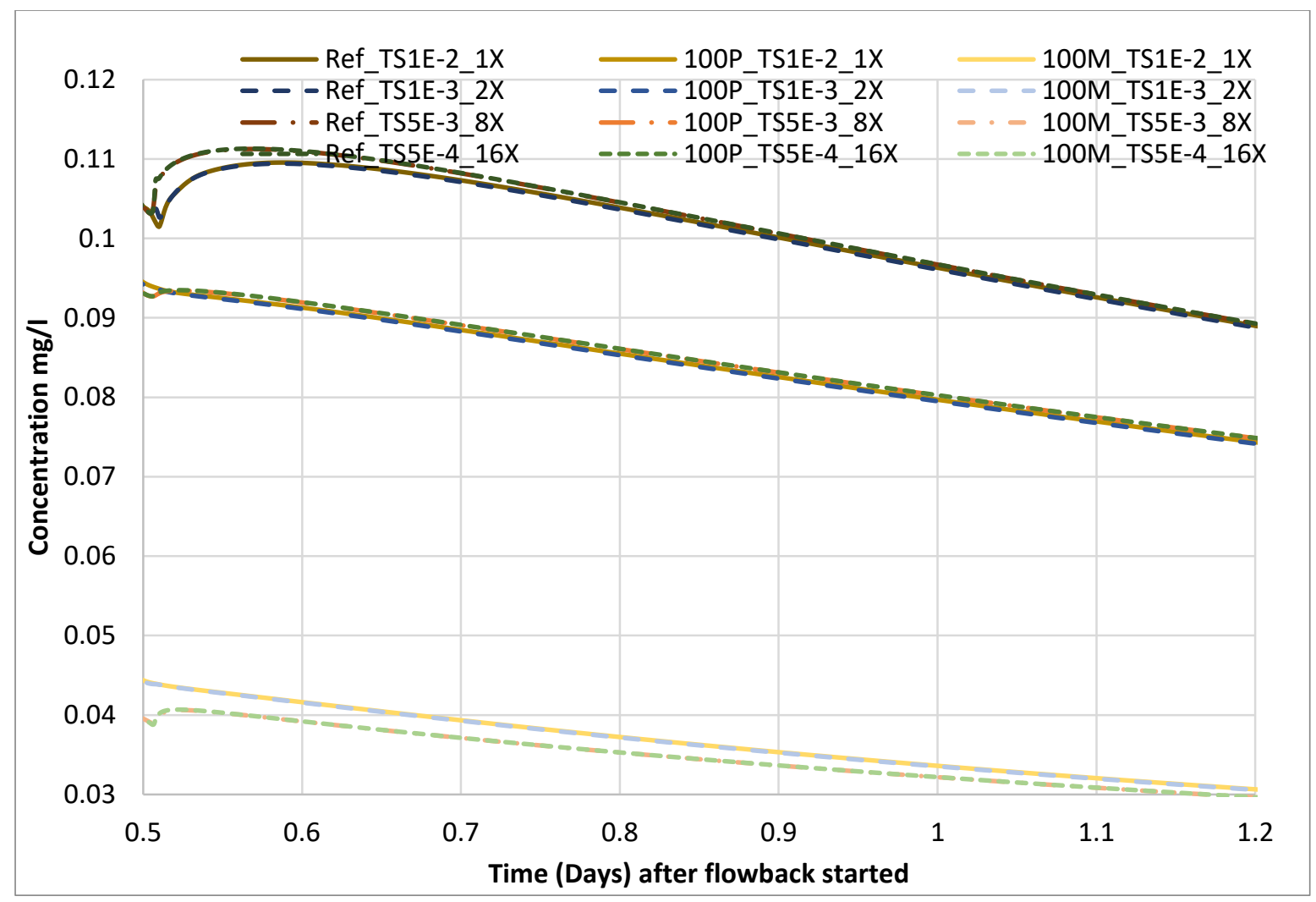

Figure 3.1.2: Spatial and temporal discretization effect on FEFLOW numerical solution single fracture tracer flowback concentrations.

The dispersion in COMSOL is implemented as Burnett and Frind (1987) tensor. The dispersivity tensor for isotropic porous medium can be defined by two constants - the longitudinal dispersivity of the medium, $\alpha \mathrm{L}$, and the transverse dispersivity of the medium, $\alpha_{\mathrm{T}}$ (Scheidegger 1961). However, in anisotropic media, the number of independent dispersivity values increases (Bear and Cheng 2010). Although conventional theory holds that $\alpha \mathrm{L}$ is an intrinsic property of the aquifer, it is found in practice to be dependent on and proportional to the scale of the measurement. Most reported values of $\alpha \mathrm{L}$ fall in a range from 0.01 to 1.0 times the scale of the measurement, although the ratio of $\alpha \mathrm{L}$ to the scale of measurement tends to decrease at larger scales (Anderson 1984; Gelhar et al. 1992). Field- scale dispersion (macrodispersion) results primarily from large-scale spatial variations in hydraulic properties (and hence in velocity). Yet one can often adequately calibrate a groundwater-flow model to observed heads using a relatively coarse grid and relatively uniform hydraulic properties that do not represent heterogeneities that affect transport. If that is the framework for the transport model, calibrations will likely lead to erroneously large values of dispersivity (e.g., Davis 1986). Similarly, representing a transient flow field by a mean steady-state flow field, as is commonly done, inherently ignores some of the variability in velocity and must be 
compensated for by using increased values of dispersivity (primarily transverse dispersivity) (Goode and Konikow 1990). Moreover, it is important to note that the sorptive tracer either proppant or matrix sorptive tracer showed similar concentration breakthrough with small difference 2E-3 which reveals a higher effect of sorption on tracer breakthrough than dispersion. Hence, to avoid or reduce the dispersion impact on the single well tracer test, a sorptive tracer would be an ideal choice for parameter estimation if sufficient sensitivity regime exists with the target parameter.

\subsection{Tracer flowback signals in COMSOL and FEFLOW from gel-proppant fracture and} water fracture, simulation concept and limitation
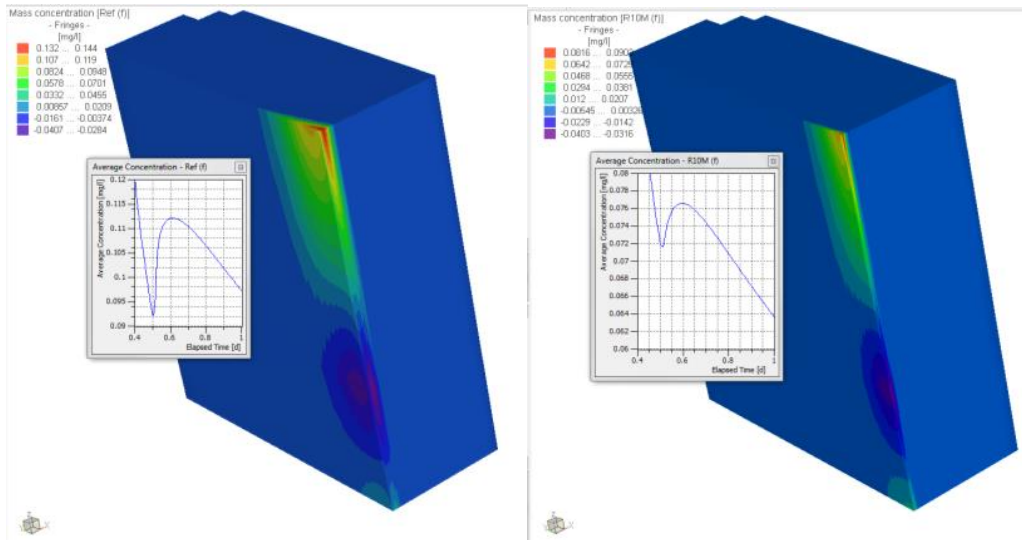

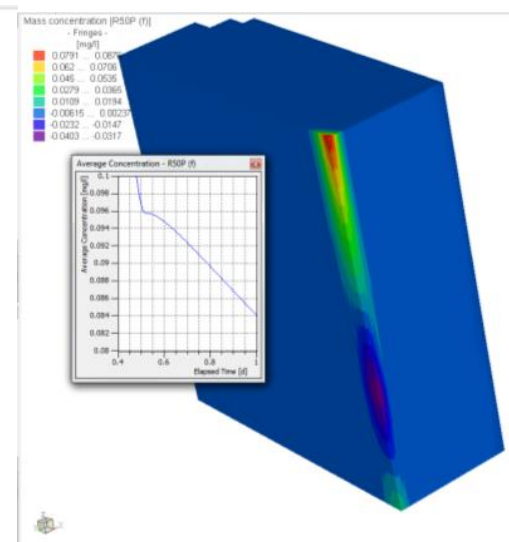

Figure 3.1.3: Tracer spreading inside the fracture and matrix during at 1 day while flowback start at 0.5 days for conservative tracer-3a (from left), low matrix sorptive tracer-3b and high MSTs- $3 c$

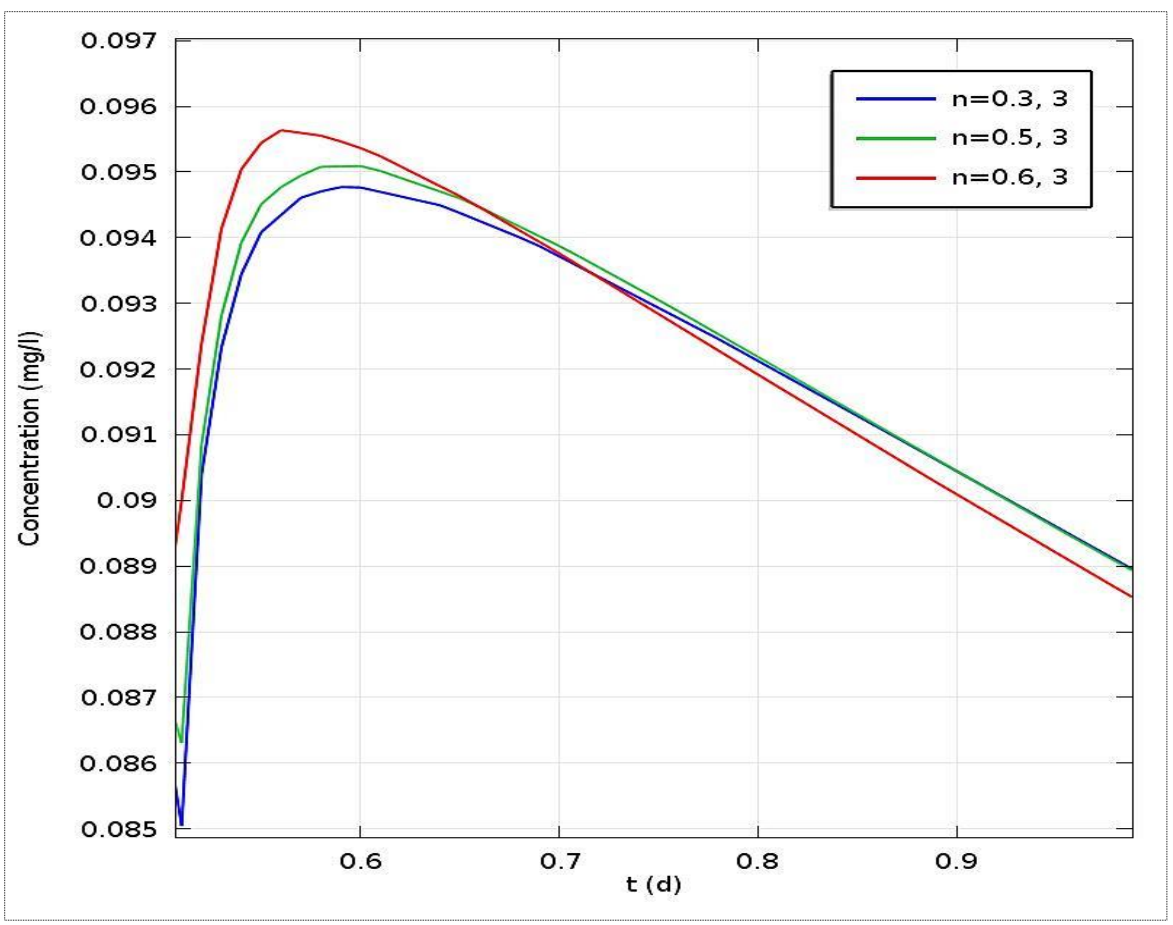




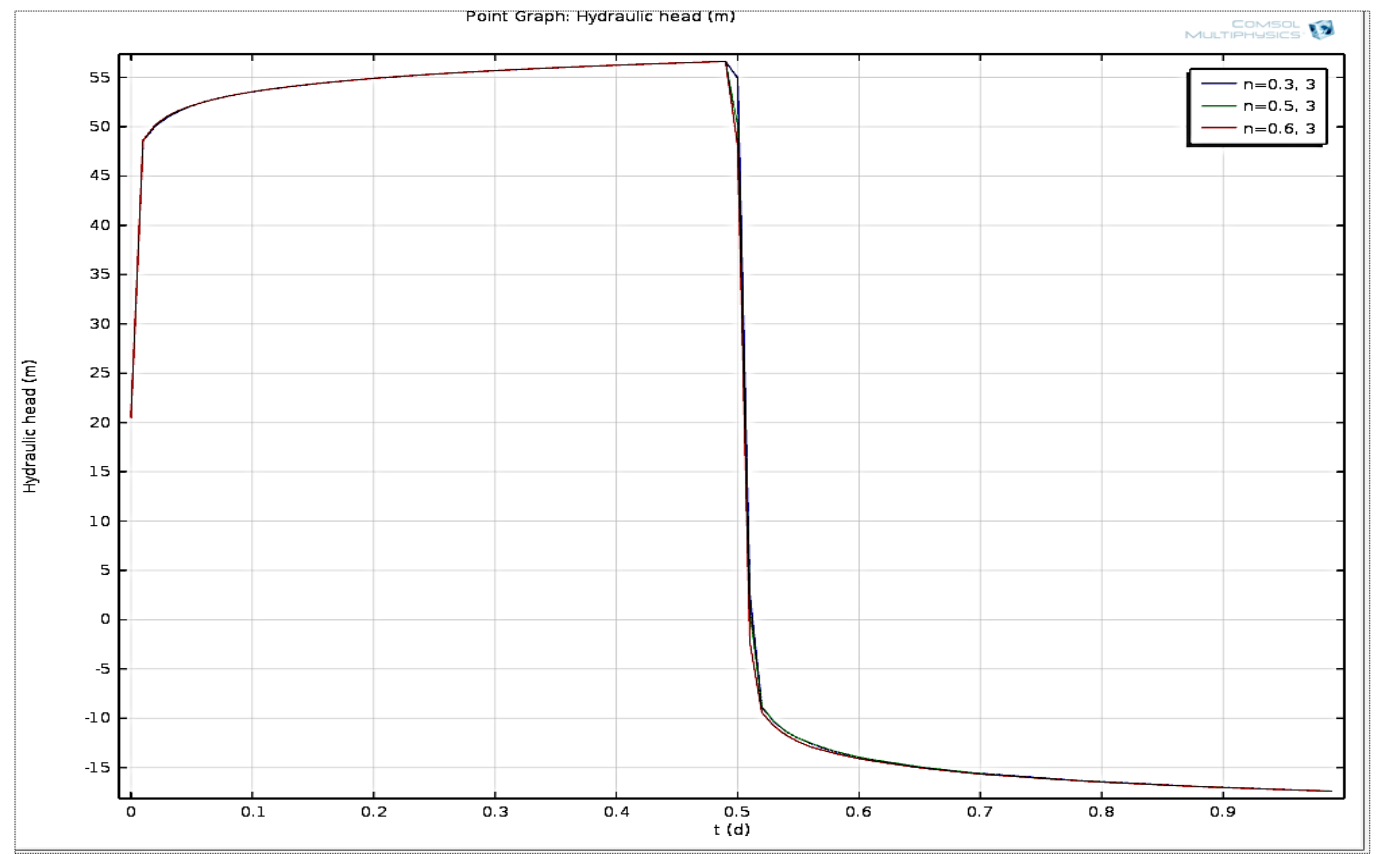

Figure 3.1.4a-b: Tracer concentration (a) and Hydraulic head (b) from different fracture porosity for injection-flowback benchmark study in single fracture-matrix domain using PDEs module in software package COMSOL Multiphysics.

Hydraulic head at the injection-flowback well showed an equal head over the whole test duration (Figure 3.1.4b). Moreover, the tracer concentration from different fracture porosity shows similar result as seen in FEFLOW simulation (Figure 3.1.4b). With the same model setup, fracture thickness result shows very different head and tracer flowback concentration than FEFLOW simulation result for same fracture and matrix parameters. This result signifies that the exchange term used in COMSOL to transfer across the fracture to matrix domain need to be review. The problem may be arising from the pressure/flow exchange term as the hydraulic head show greater anomalies (c.f. Appendix 2a).

The exchange term that implemented for fluid and solute exchange between fracture and matrix estimated as less amount of solute intrude into the matrix in COMSOL than that computed in FEFLOW, where the fracture is implemented as a discrete feature. The exchange term in COMSOL is explicitly formulated as describe in Oehlmann et al. (2015) considering the 2D exchange surface with the matrix. However, the simulation not comparable with the pressure head (shows 5 times higher) during injection and flowback simulation as well as flowback concentration is not relevant to compare. A major challenge with this translation of fractured geo-reservoir simulation has lied with the matrix and fracture exchange term, to include appropriate format fracture thickness inclusion as well as exchange pressure. However, the 
lesson from this fractured geo-reservoir simulation is extremely valuable for future code development for fractured geo-reservoir tracer flowback simulation as well as a close-form analytical solution development for solute transport.

\section{References}

Anderson, M.A. 1984. Movement of contaminants in ground- water: Groundwater transportAdvection and dispersion. In: Groundwater Contamination, 37-45. Washington, DC: National Academy Press.

Burnett, R.D. and Frind E.O., 1987, Simulation of contaminant transport in three dimensions. 2. Dimensionality effects, Water Resource Research., 23, 695-705.

Diersch, H.-J., G., 2009. FEFLOW 5.4: finite element subsurface flow and transport simulation system. Reference manual. DHI-WASY Ltd., Berlin, Germany, 292 pp

Oehlmann, S., Geyer, T., Licha, T., and Birk, S., 2013. Influence of aquifer heterogeneity on karst hydraulics and catchment delineation employing distributive modeling approaches. Hydrology and Earth System Sciences 17, 4729-4742, doi:10.5194/hess-17-4729-2013.

Oehlmann, S., Geyer, T., Licha, T., and Sauter, M., 2015. Reducing the ambiguity of karst aquifer models by pattern matching of flow and transport on catchment scale. Hydrology and Earth System Sciences 19, 893-912, doi:10.5194/hess-19-893-2015.

Scheidegger A.E. 1961. General theory of dispersion in porous media. Journal of Geophysical Research 66, no. 10: 3273-3278.

Konikow, L.F. 2011, The Secret to Successful Solute-Transport Modeling, Ground Water 49(2), $144-159$.

Konikow, L.F., W.E. Sanford, and P.J. Campbell. 1997. Constant-concentration boundary condition: Lessons from the HYDROCOIN variable-density benchmark problem. Water Resources Research 33, no. 10: 2253-2261.

Gelhar, L.W., C. Welty, and K.R. Rehfeldt. 1992. A critical review of data on field-scale dispersion in aquifers. Water Resources Research 28, no. 7: 1955-1974.

Gelhar, L.W., and C.L. Axness. 1983. Three-dimensional analysis of macrodispersion in a stratified aquifer. Water Resources Research.

Tsang, Y.W., 1992. Usage of 'Equivalent Apertures' for Rock Fractures as Derived From Hydraulic and Tracer Tests. Water Resour. Res. 28(5), 1451-1455. 
CHAPTER 3-Part 2: A set of benchmark studies on flow and solute transport in geological reservoirs

Shyamal Karmakar, Alexandru Tatomir, Sandra Oehlmann, Markus Giese, Martin Sauter

\section{Citation:}

Karmakar, S., Tatomir, A., Oehlmann, S., Giese, M., Sauter, M., 2016, A set of benchmark studies on flow and solute transport in geological reservoirs, Prepared for the journal Computational Geoscience and Modeling, 2016

Geoscience Centre of the University of Göttingen, Department of Applied Geology,

Goldschmidtstraße 3, 37077 Göttingen, Germany

*Corresponding author: shyamal.karmakar@geo.uni-goettingen.de 


\begin{abstract}
Modeling groundwater flow and solute transport are important for a set of engineering and hydrogeological applications. One of the most common examples is the designing of tracer tests used for the characterization of geological reservoirs. The quality of the prediction to be made with a numerical simulator is highly dependent on the quality of the numerical simulator itself. Besides the accuracy of the results, aspects like financial costs of the simulation software and the computer resources needed for simulation have to be taken into account. This study gives an evaluation of different numerical models/approaches and outlines the advantages and disadvantages of several numerical solution software packages in terms of solution predictability, temporal control, and computational efficiency. A set of well-established software frequently used for modeling flow and transport in geological reservoirs is tested and compared (MODFLOW/MT3DMS, FEFLOW, COMSOL Multiphysics and DuMu'). Those modeling tools are based on different numerical discretization schemes i.e. finite differences, finite volumes and finite element methods. The influence of dispersivity, which is directly related to the numerical modeling, is investigated in parametric studies and results are compared with analytical approximations. At the same time, relative errors are studied in a complex field scale example. From this comparative study, it is revealed that under forced gradient conditions, finite element codes COMSOL and FEFLOW show a higher accuracy with respect to the analytical approximation for a certain range of dispersivity than $\mathrm{DuMu}^{\mathrm{x}}$ and MODFLOW/MT3DMS. Comparing simulation time and code parallelization, FEFLOW performs better than COMSOL. Overall computational time is lowest for finite difference software MODFLOW/MT3DMS for a small number of mesh elements $(\sim$ less than 12800 elements). For large meshes (>128000 elements), finite element software FEFFLOW performs faster.
\end{abstract}

\title{
1 Introduction
}

Benchmarking numerical software for fluid flow and solute transport is a state of the art for decades. Usually, the developers of a software or analytical approximation verify and optimize their software or codes through conducting a number of forward simulations for a set of benchmark problems (e.g. HYDROCOIN, Ogata and Bank, 1961, Zheng and Wang 1999, Diersch 2009, Kolditz 2016). These benchmarks show the accuracy of the numerical solver with respect to established analytical or approximate solutions. Most of the software's user manuals or reference books (e.g. Diersch, 2013, Zheng and Wang, 1999) include example applications with benchmark problems showing solution accuracy. Further, some developers 
present and document user's experiences on their web-pages or in newsletters. There are also independent studies such as 'software spotlight' in the journal 'Groundwater', which discuss individual software installation, implementation of parameters and boundary condition as well as performances.

Some benchmarks also include software applicability, e.g. solution efficiency, code parallelization, resource uses or user friendliness. However, these usually refer only to a single software package and do not allow for comparison between different numerical codes (e.g., Class et al 2009). The benchmark simulations given for the different software packages differ in the problem setup, e.g. in terms of spatial discretization or mesh type, making it difficult to compare them. Further, aspects like financial cost are not taken into account. This makes it difficult for the user to decide on the numerical simulator to be used for a certain problem.

The partial differential equations describing groundwater flow and transport can be solved mathematically using either analytical solutions or numerical solutions. For most field problems, the mathematical benefits of obtaining an analytical solution are probably outweighed by the errors introduced by the simplifying assumptions for the complex field environment that are required to apply the analytical model. In most aquifers the most important parameter controlling transport is hydraulic conductivity, through its control of velocity (equation 2). Consequently, solute spreading will be represented by local differences in advection, so uncertainty in estimating dispersivity and conceptual flaws in the mathematical representation of the dispersion process will matter less. The dispersivity parameter is conceived as a measurable physical parameter that primarily reflects the nature of the heterogeneity in the system, where dispersivity determines the spreading of solutes in the porous media. However, numerical dispersion occurs during the numerical solution process due to spatial and temporal discretization. From the numerical solution breakthrough curves, it is difficult to distinguish these two types of dispersion. Therefore, the sensitivity of numerical simulation results with the dispersivity values is studied in three benchmark problems.

In this study, different factors for selecting software are considered. FEFLOW and MODFLOW/MT3DMS are the most common ones highly dedicated to porous media flow and solute transport. They are compared with COMSOL Multiphysics and DuMux, which were developed for extended applications but are also applicable for single phase flow and solute transport in porous media. MODFLOW is based on the finite difference method for spatial discretization, which is the most popular and pioneered in numerical simulations of fluid flow. 
FEFLOW and COMSOL Multiphysics both are based on the finite element method, which allows for more flexible meshing. Lastly, DuMux is an academic free open source software based on the finite volume method dedicated to multiphase flow and transport problems. The software packages are compared with respect to solution accuracy, efficiency, i.e. time and computer resources needed, user friendliness and financial cost.

\section{Methodology}

\subsection{Mathematical model}

The flow equations that are solved in the analytical approximation and the numerical software are:

$$
\begin{gathered}
S_{0} \frac{\partial p}{\partial t}+\nabla \cdot q_{i}-Q_{p}=0 \ldots \ldots \\
q_{i}=-\left\{\rho \frac{\mathbf{K}}{\mu}(\operatorname{grad} p-\rho \mathbf{g})\right\}
\end{gathered}
$$

$S_{0}\left[\mathrm{~L}^{-1}\right]$ is the storage term, $\mathbf{K}\left[\mathrm{L}^{2}\right]$ is the intrinsic permeability, $\mathrm{p}\left[\mathrm{ML}^{-1} \mathrm{~T}^{-2}\right]$ is pressure, $q_{i}$ $\left[\mathrm{LT}^{-1}\right]$ is the Darcy velocity vector, $x_{i}$ is the spatial discretization vector, $Q_{p}$ the source or sink for fluid, $\mu\left[\mathrm{ML}^{-1} \mathrm{~T}^{-1}\right]$ the dynamic viscosity, $\rho\left[\mathrm{ML}^{-3}\right]$ the density of fluid, and $\mathrm{g}\left[\mathrm{LT}^{-2}\right]$ the acceleration due to gravity.

Transport is described by an advection-dispersion equation of divergent form that is solved in the numerical software as follows:

$$
\begin{array}{r}
\frac{\partial C}{\partial t}-\nabla \cdot\left\{v C+D_{i j} \operatorname{grad} C\right\}+R \\
\frac{\partial}{\partial t}(R C)+\nabla q C-\nabla\left(\phi D_{i j} \nabla C\right)-Q_{c}-R_{k}=0
\end{array}
$$

$\mathrm{R}[]$ is the retardation, $q_{i}\left[\mathrm{LT}^{-1}\right]$ is the Darcy velocity vector, $Q_{c}\left[\mathrm{ML}^{-2} \mathrm{~T}^{-1}\right]$ the source or sink for solute, $R_{k}\left[\mathrm{ML}^{-2} \mathrm{~T}^{-1}\right]$ reaction rate, $D_{i j}-[\mathrm{L}]$ dispersion vector, $\mathrm{C}\left[\mathrm{ML}^{-3}\right]$ volumetric concentration.

In case of radial symmetry (for problem 2, 2D) the transport equation (after Schroth et al 2001) can be written as 


$$
\frac{\partial C}{\partial t}+\frac{\rho_{b}}{n} \frac{\partial S}{\partial t_{x}}-D_{L} \frac{\partial^{2} C}{\partial r^{2}}+q_{i} \frac{\partial C}{\partial r}+\lambda C=0
$$

Here $\mathrm{C}\left[\mathrm{ML}^{-3}\right]$ - volumetric concentration, $\rho_{b}\left[\mathrm{ML}^{-3}\right]$-density of solid in porous media, nporosity, $\mathrm{S}$-storage coefficient, $D_{L}-[\mathrm{L}]$ longitudinal dispersion, and $\mathrm{r}$ the distance as radial coordinate from injection well, $\lambda\left[\mathrm{ML}^{-3} \mathrm{~T}^{-1}\right]$ - degradation or decay rate of the solute and $\mathrm{v}\left[\mathrm{LT}^{-}\right.$ ${ }^{1}$ ]- the Darcy velocity. In an REV only one velocity exists in time and space, therefore, velocity fluctuations within the REV are neglected. They are taken into account within the transport equation by the concept of mechanical dispersion.

The numerical simulators results are compared with the analytical solution for the 1D and 2D problems that are described in the section below.

The analytical solution for the 1D problem was given by Ogata and Banks (1961),

$$
c(x, t)=\frac{1}{2}\left\{\operatorname{erf} c\left[\frac{x-v t}{2 \sqrt{D_{L} t}}\right]+\exp \left[\frac{v x}{D_{L} t}\right] \cdot \operatorname{erf} c\left[\frac{x+v t}{2 \sqrt{D_{L} t}}\right]\right.
$$

The analytical solution for the 2D problem was given by Gelhar and Collins (1971), which was adapted and modified after Schroth et al (2001) as follow

$$
\frac{C}{C_{0}}=\frac{1}{2} \operatorname{erfc}\left[\frac{\left(r^{2}-r_{i n j}^{2}\right)}{\sqrt{\frac{16}{3} \alpha_{L} r_{i n j}^{3}}}\right], \text { where } r_{i n j}=\sqrt{\frac{Q t}{\pi n R}}
$$

Where, $\mathrm{v}\left[\mathrm{LT}^{-1}\right]$ - the Darcy velocity, $\mathrm{Q}\left[\mathrm{L}^{3} \mathrm{~T}^{-1}\right]$ - the volumetric flow rate, $\mathrm{r}_{\mathrm{inj}}[\mathrm{L}]$ radial distance that the solute travels during injection, $\mathrm{r}[\mathrm{L}]$ radial distance of the observation point from the injection well, $D_{L}-[\mathrm{L}]$ longitudinal dispersion, $\alpha_{L}[\mathrm{~L}]$ longitudinal dispersivity, $\mathrm{Q}\left[\mathrm{L}^{3} \mathrm{~T}^{-1}\right.$ ] injection rate, R [] retardation and $\mathrm{n}[\mathrm{]}$ porosity. For conservative tracer, R [] is equal to 1.

\subsection{Problem definition}

Three benchmark problems with gradually increasing geometrical complexity (i.e., 1D, 2D, and 3D) are defined for this study. A brief description of the problems is given, including the model geometry, input parameters, boundary conditions and simulation period, in the subsection below. The structured grid was chosen from the different mesh types available in the participating software packages, to keep the degrees of freedom to be solved by the simulators identical. Problem definition for 1D, 2D and 3D were formulated and solved for flow and solute transport. The computational performance of the different simulators was 
studied for the 2D and 3D problem cases. For 1D simulation time was relatively very short and did not vary significantly among the simulators.

\subsubsection{Problem 1: 1D - Solute tracer transport for steady state flow with a forced head gradient in a homogenous aquifer}

This benchmark problem and the analytical solution were formulated by Ogata and Banks (1961). The model domain is defined by a hydraulic head gradient where a constant concentration source of conservative solute is present at the higher head boundary (left side) and advective flow carries the solute to the observation point (Figure 3.2.1). Flow and solute transport are simulated for a $100 \mathrm{~m}$ long column with a constant head gradient of 0.02 for a homogenous and isotropic porous medium pertaining a conductivity of $1 \times 10-4 \mathrm{~m} / \mathrm{s}$ and $25 \%$ porosity. The simulation period is 200 days. The time step is set as one day (maximum time step size) and the domain is discretized using a mesh size $1 \mathrm{~m}$. The observation point is located at a distance of $50 \mathrm{~m}$ from the point fixed concentration solute source. For the sensitivity study, different dispersivity values are simulated.

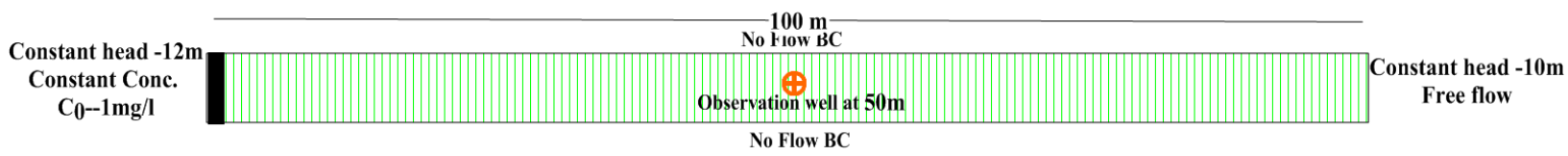

Figure 3.2.1: Problem 1: 1D model domain assuming a free flow boundary at right end and higher gradient at left end with a constant point contaminant source.

\subsubsection{Problem 2: 2D-Solute transport in a confined homogenous aquifer from a forced gradient point source}

The benchmark problem considered in this section includes the concentration change at an observation well during injection at a fully penetrating well in a confined aquifer. Constant solute concentration, $\mathrm{Co}$, is assumed at the injection well. Moreover, constant head boundaries are imposed at the other three sides of the domain assuming that it would impose a "free outflow' boundary during the simulation period. Simulation period is 200 days. The time step is set as one day (maximum time step size). Storage is set to zero so that steady state flow conditions are achieved instantaneously after the injection. Since a symmetric radial flow and transport behavior is expected for the stated model set up, the numerical models that are set up study only half of the domain assuming symmetry at the middle of the domain at the injection point. 
A numerical model consisting of 40 columns, 20 rows was implemented in all of the participating software platforms to simulate the concentration breakthrough at the observation point at $25 \mathrm{~m}$ distance from the injection well. The numerical simulators results were compared with the analytical approximation given by Gelhar and Collins (1971), which was adapted and modified after Schroth et al (2001).

The initial and boundary conditions, as well as model domain flow and transport properties, are shown in Figure 3.2.1.

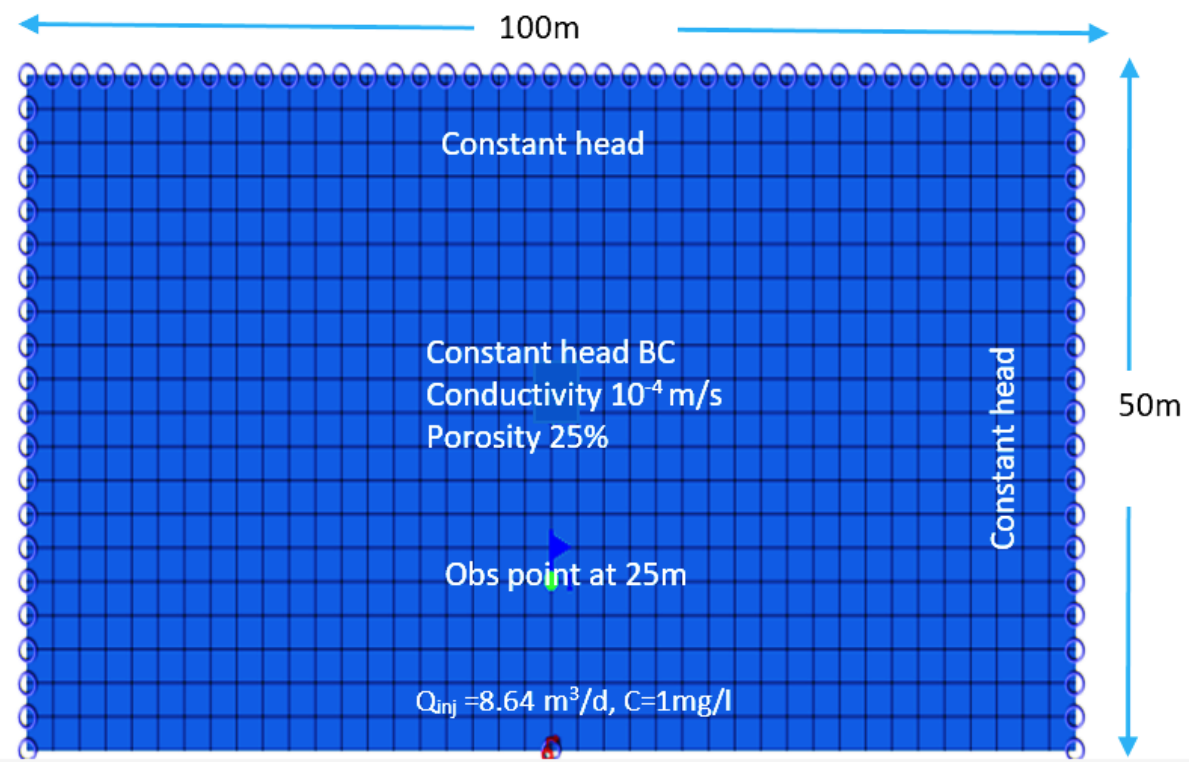

Figure 3.2.2: Problem 2: $2 D$ domain assuming a radial symmetry of flow and solute at the injection point of solute located the middle of the domain. Hence, half of the domain is taken into account for the numerical simulation, with the injection well and point source applied at the middle point of the lower border of the domain and the rest of the lower border constrained as no-flow boundary.

\subsubsection{Problem 3: 3D-Solute transport for confined homogeneous multi-layered forced gradient conditions}

This section describes the application of a field scale problem involving the evaluation of tracer test in a dipole-forced gradient situation with multilayered injection and pumping wells. The discussion is intended to demonstrate the performance of all four participating codes in a 3D problem commonly encountered in reservoir engineering i.e., a layered aquifer with alternating layers of high and low permeability and porosity. The models represent a geological crosssection with five layers of different thickness (Tatomir et al. 2016). The lateral extent of the domain is $100 \mathrm{~m} \times 100 \mathrm{~m}$. Three of the layers represent hydraulically conductive sandstone aquifers $(8.03 \times 10-8 \mathrm{~m} / \mathrm{s}, 1.97 \times 10-7 \mathrm{~m} / \mathrm{s}, 4.36 \times 10-8 \mathrm{~m} / \mathrm{s}$ from top to bottom). They are separated by relatively less permeable layers or aquitards $\left(9.69 \times 10^{-12} \mathrm{~m} / \mathrm{s}\right)$ of $1 \mathrm{~m}$ thick silty 
clay lenses. The geologic setting of the study site is illustrated in Figure 3.2.3. Thick clay lenses are also assumed above and beneath the modeled formation. Those layers are assumed to be impermeable; hence, they are represented by 'no flow' type boundaries on top and bottom of the model domain. The porosity values $14.5 \%, 16.3 \%$ and $13.3 \%$ for the permeable sandstone and 3.9\% for the aquitards (Figure 3.2.3) are used. Injection and pumping wells are imposed in the three conductive layers and each layer is discretized with a uniform thickness of $0.5 \mathrm{~m}$. For the numerical simulation, symmetry is assumed and only half of the domain is modeled. The model is discretized with a square mesh of $2.5 \mathrm{~m}$. The injection rate and pumping rate is 0.432 $\mathrm{m}^{3} /$ day at each point (a sum of $8.64 \mathrm{~m}^{3} /$ day) as shown in Figure 3.2-3. The time-concentration data from these simulation results are compared against MODFLOW/MT3DMS data. Simulation period is 200 days. The time step is set as 0.5 days (maximum time step size). As the time stepping was found different in different simulation packages, simulation result efficiency comparisons are done using 'cubic interpolation' (a third-degree polynomial) method in Matlab (http://www.mathworks.com/) to interpolate the data and calculate the RMSE between the curves.

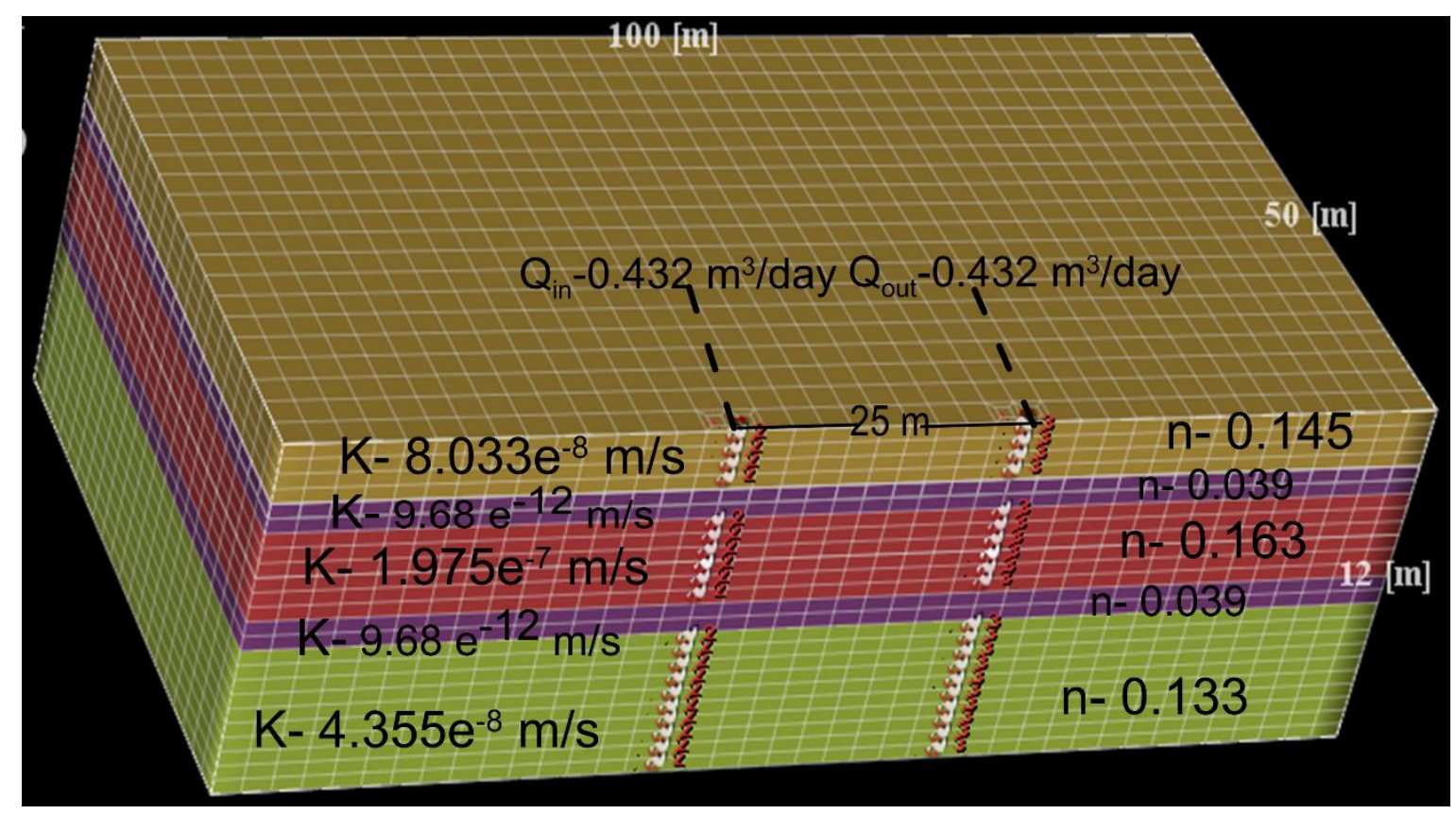

Figure 3.2- 3: 3D model domain, showing the rectangular mesh and the permeability and porosity distribution over the layers. Left side points: injection points; right side points: pumping well.

\section{Benchmarking simulators}

Parametric studies on dispersivity and permeability are investigated for the $3 \mathrm{D}$ problem. At the same time, computational efficiency of different software packages is tested on a Linux 
platform 4-core $2.32 \mathrm{GHz}$ processor CPU with $8 \mathrm{~GB}$ RAM memory computer. Among the participating software, parallel computing for both flow and solute transport modules is only supported in COMSOL and FEFLOW. Therefore, for these two software packages, computational performance is studied for both single core and multi-core computing.

\subsection{MODFLOW/MT3DMS}

MODFLOW is a finite difference method based software which is commonly applied over decades for fluid flow and solute transport (Harbaugh et al. 2000). As an open source software distributed by US Geological Survey (USGS) it is freely available but also modified and revised commercial generic versions such as Visual MODFLOW, PMWIN and GMS are used. The USGS also provides a pre- and post-processing GUI called ModelMuse, which is also freely available. For the following analysis, ModelMuse GUI has used for model building and simulation result visualization. The finite difference based space and time discretization method is its major advantages which can be expected to cost relatively low memory and simulation time. The Flow Model Interface Package of MT3DMS reads the saturated thickness, fluxes across cell interfaces in all directions, locations and flow rates of the various sources and sinks from an unformatted flow-transport link file saved by a flow model used in conjunction with the MT3DMS transport model. If the flow model, MODFLOW, is used for flow simulation, a package named LKMT3 (where 3 denotes the version number) in addition to MODFLOW is included with the MT3DMS distribution files. For this flow simulation study, the preconditioned conjugated gradient solver (PCG) in MODFLOW with layer centered grid and generalized conjugate gradient solver (GCG) in MT3DMS for solute transport are used using the TVD method (a third order total-variation-diminishing (TVD) scheme for solving the advection term).

\subsection{FEFLOW 6.0}

FEFLOW (Finite Element subsurface FLOW and transport system) is an interactive groundwater modeling software for $2 \mathrm{D}$ and $3 \mathrm{D}$ fully coupled or uncoupled, thermo-hydrochemical (THC) processes in saturated or variably saturated systems. The reactive multispecies transport can be modeled in subsurface water environments with or without one or multiple free surfaces. The option to use and develop user-specific plugins via the programming interface (Interface Manager IFM) allows the addition of external code or even external programs to FEFLOW. For example, by adding the newly developed mechanical plugin it becomes a THMC simulator. It is endowed with a user-friendly model builder interface. 
Besides the parallelized (OpenMP) computational core, it has also powerful pre- and postprocessing capabilities, including 2D and 3D GIS data, to name some. FEFLOW is available for WINDOWS systems as well as for different Linux distributions. The current version, FEFLOW 7.0, which takes over the classic version FEFLOW 6.0, includes a few new features such as multi-layer wells (would be useful for multilayered-well implementation) with a new GUI and parameter visualization. However, for this study, FEFLOW 6.0 is used which is the transitional package that contains both standard new interface as well as 'classic interface'. Additional information about FEFLOW is available at http://www.mike-wasy.com. The 3D multilayered well georeservoir simulation in this benchmark study uses well boundary conditions that do not act as multilayer wells. Instead, separate well BC for each layer is introduced.

\subsection{COMSOL Multiphysics 4.4}

COMSOL Multiphysics (formerly known as FEMLAB) is a finite element software for numerical simulations of various physical applications. It is mainly employed for technical applications, but also receives increasing attention for environmental issues during recent years (e.g. Joodi et al. 2010, Jin et al. 2014, Oehlmann et al. 2013, 2015). In addition to a number of available interfaces for standard applications, e.g. flow in porous media, COMSOL offers the possibility to implement individual Partial Differential Equations without needing access to the source code. This makes COMSOL highly flexible. The software focuses on the interconnection of different physical processes allowing for multiphysical and multidimensional couplings. COMSOL can be purchased for Windows, Mac, Linux or UNIX systems. It offers a number of direct and iterative solvers to choose from for the various applications. Interfaces to external software, e.g. MathWorks MATLAB (http://www.mathworks.com/) allow for easy transfer of model results and geometries. However, limited pre- and post-processing possibilities are also available in COMSOL itself. The Subsurface Flow Module, which is used in this study, offers standard equations for flow and transport in saturated and unsaturated porous media. Flow and transport are treated by separate interfaces. The groundwater flow velocities derived by the flow interface can directly be used as input for the transport interface. The equations can then either be solved simultaneously or step-wise reducing simulation time. For this study, the preconditioned conjugated gradient (PCG) solver is used since this is also the standard solver in FEFLOW. 


\section{$3.4 \mathrm{DuMu}^{\mathrm{x}}$}

The one phase flow two component transport numerical model, $1 \mathrm{p} 2 \mathrm{c}$, implemented in $\mathrm{DuMu}^{\mathrm{x}}$ (Flemisch, et al 2011) is used. The free and open source academic numerical model is available to download at www.dumux.org from a series of ready-to-use models. The Department of Hydromechanics and Modeling of Hydrosystems, University of Stuttgart, in-house simulator $\mathrm{DuMu}^{\mathrm{x}}$ is a multi-scale, multiphysics toolbox for the simulation of flow and transport processes in porous media. The toolbox is based on the Distributed and Unified Numerics Environment (DUNE) (Bastian et al 2008a, b). DuMu ${ }^{\mathrm{x}}$ comes in the form of an additional module and inherits functionality from all available DUNE modules. Its main intention is to provide a framework for easy and efficient implementation of models for porous media flow problems, ranging from problem formulation, selection of spatial and temporal discretization schemes and non-linear solvers, up to general concepts for model coupling. For the space discretization, a BOX scheme is used, while the time integration is performed via a standard implicit Euler scheme. A Newton-Raphson method with an adaptive time step selection solves the non-linear equations in each time step.

\section{Result}

The results of the benchmark problems are described in the following sections.

\subsection{Problem 1: 1D - Solute transport in a homogeneous aquifer with a natural gradient}

The analytical solution for concentration breakthrough at $50 \mathrm{~m}$ distance from a point source and the numerical solution simulated for same conditions are presented in Figure 4 for two dispersivity cases viz. $0.7 \mathrm{~m}$ and $5 \mathrm{~m}$. The two distinct sets of curves for different dispersivity value revealed that all participating simulators, as well as the analytical solution, are sensitive to this important hydrogeological parameter. Hereby, the dispersivity of $5 \mathrm{~m}$ is used as a standard scenario for the observation at $25 \mathrm{~m}$ from injection point for 2D and 3D case. Since, it represents a median value that falls within a range from 0.01 to 1 times 'scale of measurement' that can encounter in the field (Anderson 1984; Gelhar et al. 1992). For this value, the accuracy of all numerical simulators on the analytical solution (after Ogata and Bank, 1961) was judged to be good with an RMSE of less than $0.1 \mathrm{mg} / \mathrm{l}\left(10 \%\right.$ of $\left.\mathrm{C}_{0}\right)$. Figure 3.2.5 gives the RMSE of the breakthrough curves at the observation well predicted by the participating software packages in problem 1 for different dispersivity value. 


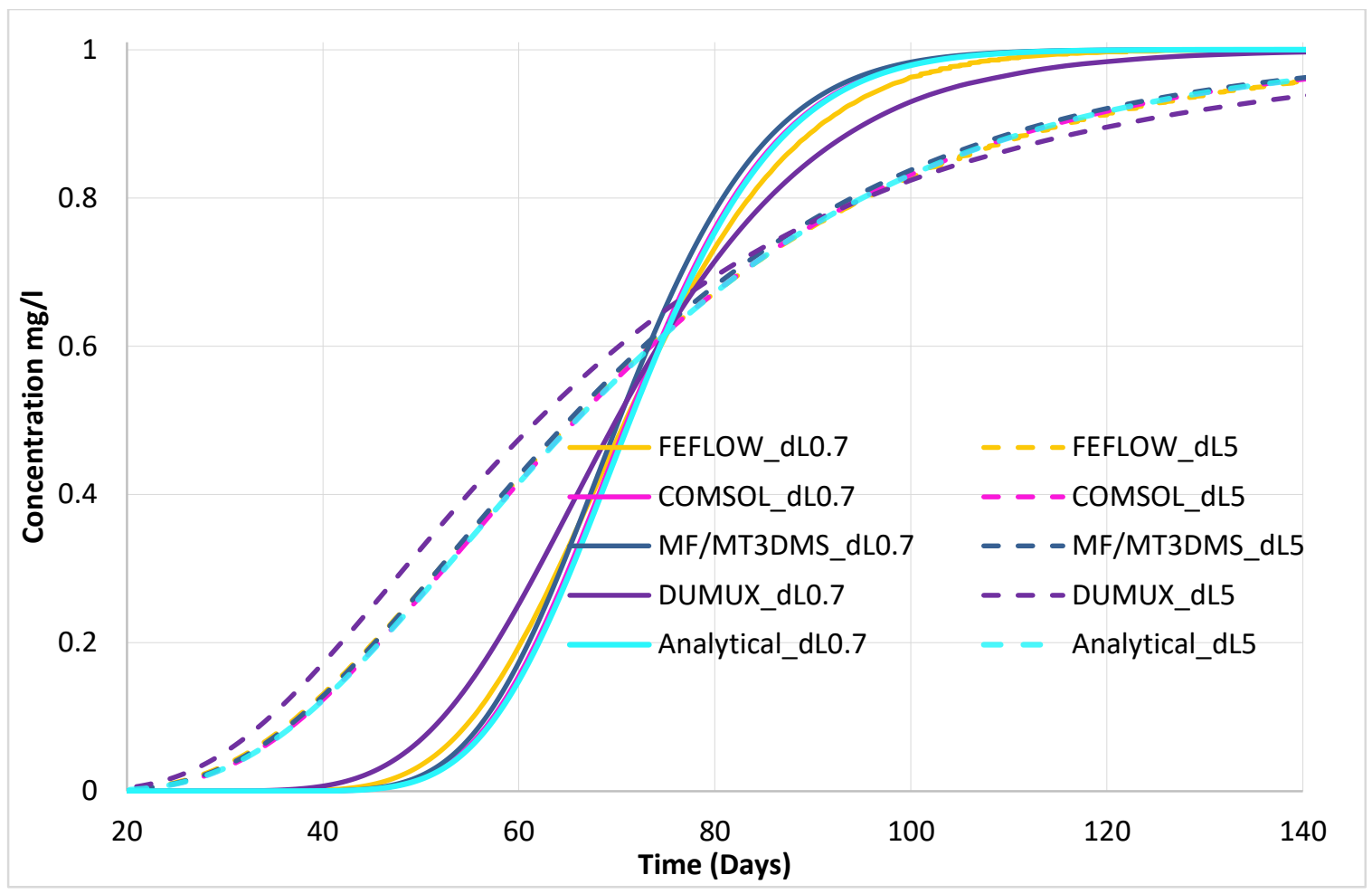

Figure 3.2.4: Problem 1, tracer breakthrough from various solvers with dispersivities $5 \mathrm{~m}$ and $0.7 \mathrm{~m}$.

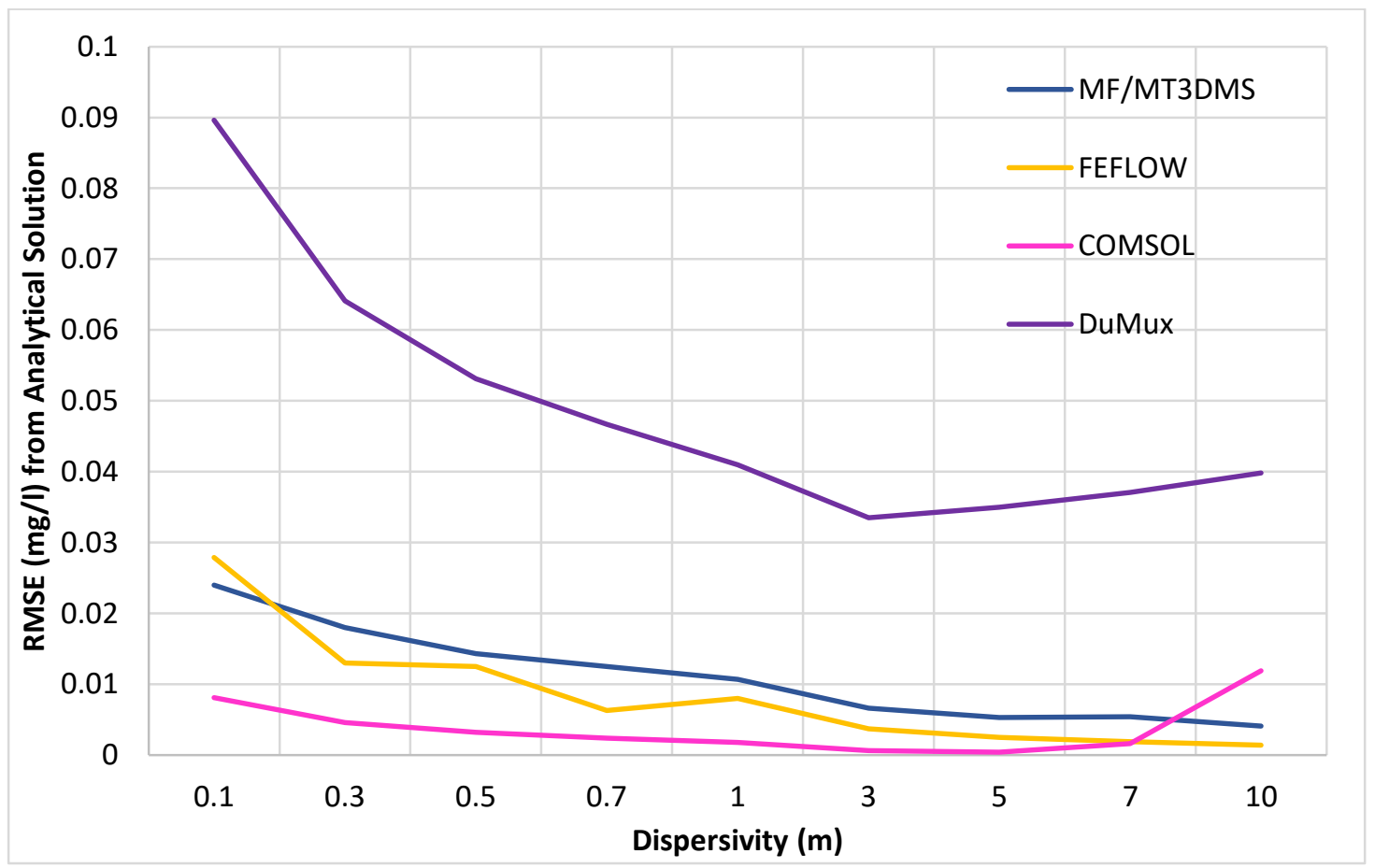

Figure 3.2.5: The relative difference between the numerical and analytical solutions within the simulated range of dispersivity values.

The lower dispersivity case, here $0.7 \mathrm{~m}$, shows higher differences between the simulated breakthrough curves. This effect is especially pronounced for DuMu${ }^{\mathrm{x}}$. It is important to note 
that the RMSE significantly decreases at higher dispersivity values (c.f. Fig. 3.2.4 and Fig 3.2.5). DuMux ${ }^{\mathrm{x}}$ shows high RMSE values for dispersivity of less than $3 \mathrm{~m}$ while the errors are similar to the other software packages for higher dispersivity values. However, all errors (maximum value) lay in a range of $2-8 \%$ of $\mathrm{C} 0$, being well beneath the threshold of $10 \%$ of peak concentration, meaning the accuracy is sufficient.

\subsection{Problem 2: 2D-Solute transport in forced gradient homogeneous aquifer}

This problem includes solute transport from an 'injection well' to the porous medium in a homogeneous and confined aquifer. Tracer concentration is observed at $25 \mathrm{~m}$ distance from the injection well. The concentration over time curve from different software packages revealed a significant difference during the 200 days of simulation period (result presented for 120 days in Figure 3.2.6). The relative error with respect to the analytical solution is estimated using standard root mean squared error method (RMSE). However, with increasing dispersivity, the numerical simulators breakthrough curves show the lower difference with analytical solution (Figure 3.2.7). This is particularly true for the case for $\mathrm{DuMu}^{\mathrm{x}}$, for which the good convergence comes at the cost of significant numerical dispersion for low dispersivity values.

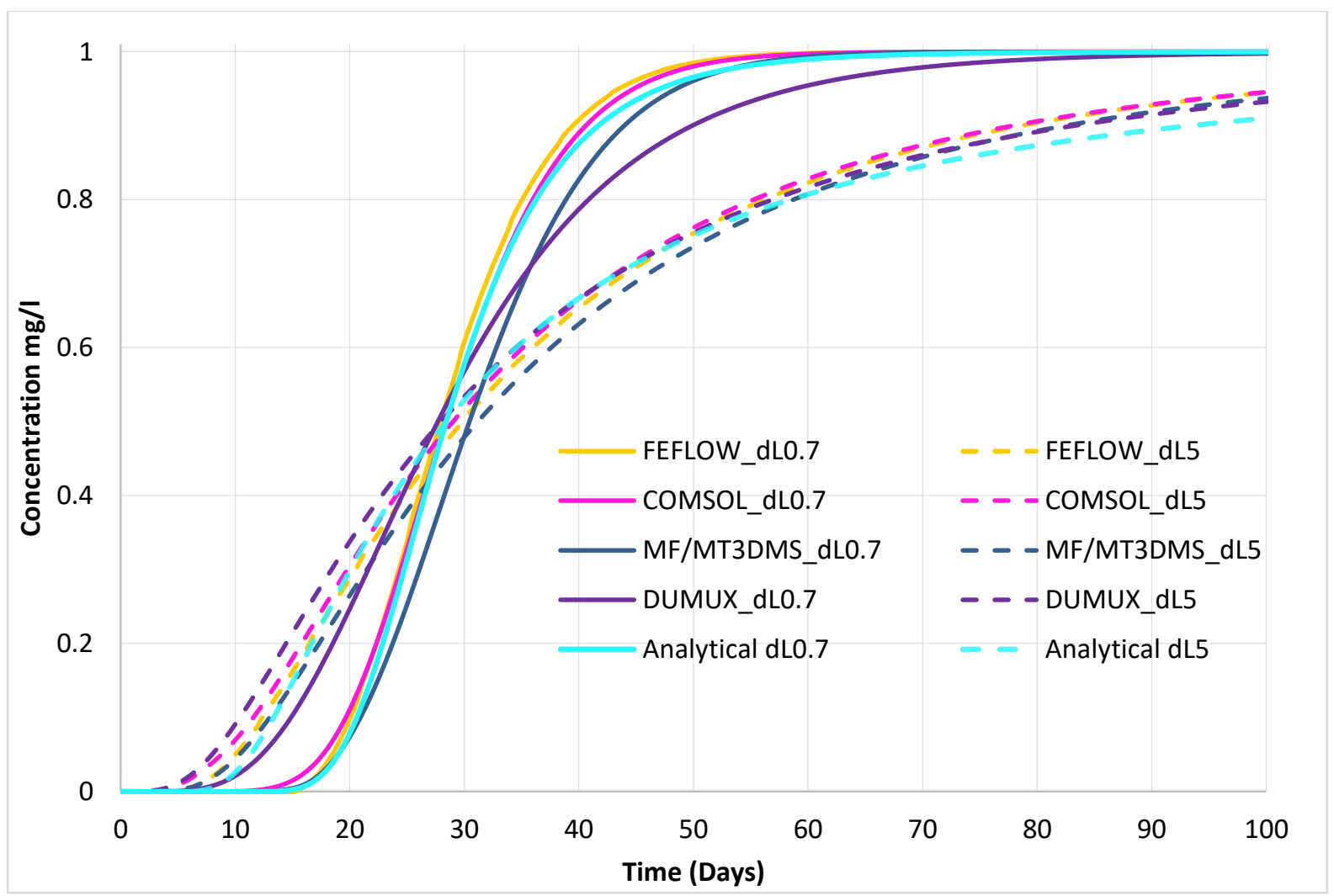


Figure 3.2.6: Time- concentration curve for two different dispersivity value $0.7 \mathrm{~m}$ and $5 \mathrm{~m}$ simulated in MODFLOW-MT3DMS, FEFLOW, COMSOL and DuMu for problem 2D and analytical solution from Gelhar and Collins (1971).

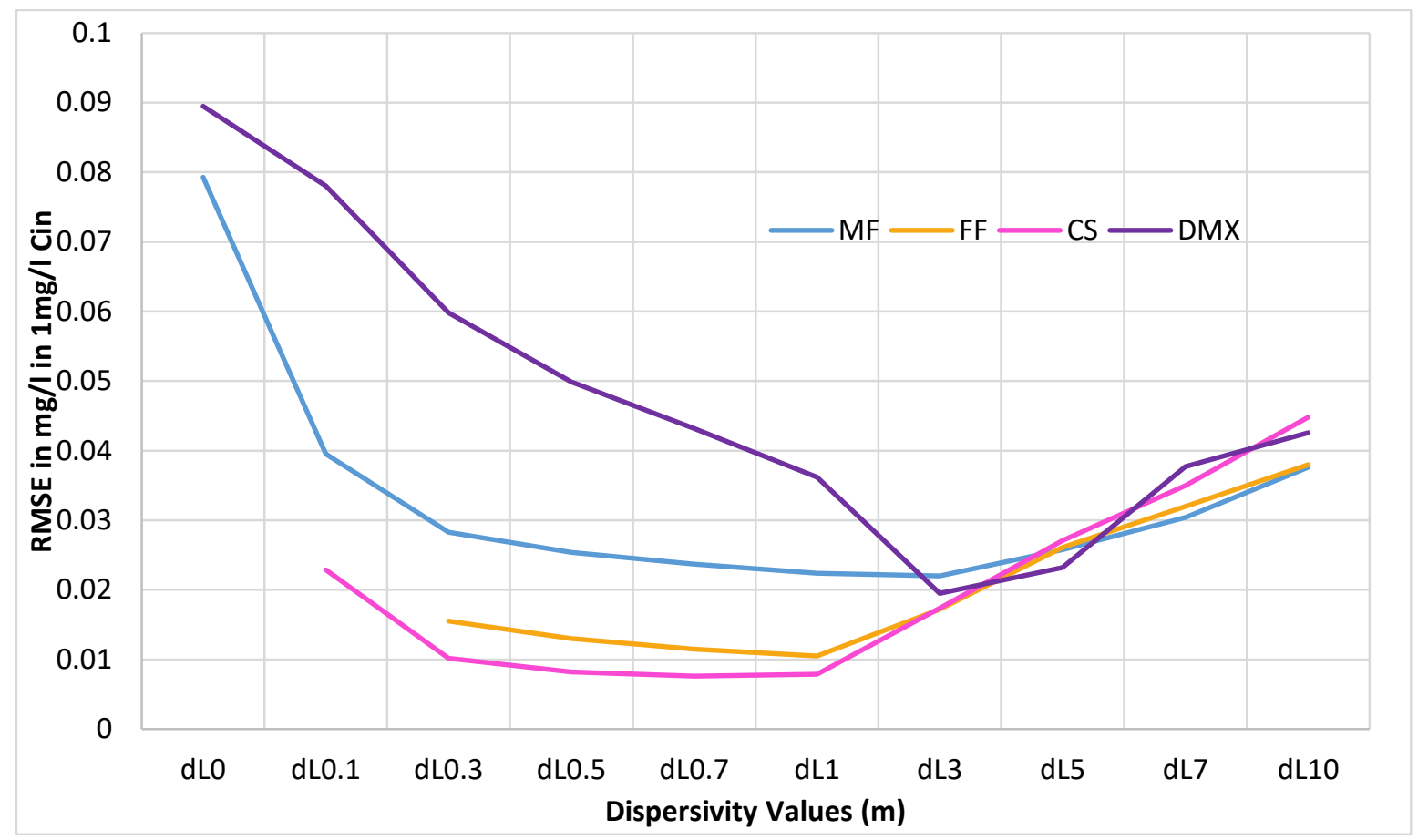

Figure 3.2.7: The relative difference of the numerical solution from the analytical solution for different dispersion value for benchmark problem 2: 2D.

\subsubsection{Spatial discretization effects on solution efficiency}

Spatial discretization is most of the time a 'trade-off' between the accuracy of the results and 'resource cost'. Here resource cost implies, the computer virtual memory requirement, simulation time, Figure 3.2.7 shows the influence of the spatial discretization on the accuracy of the solution for our benchmark problem 2, were sufficiently refined to simulate the result with a reasonable accuracy for the participating software. Furthermore, all simulators perform better with a mesh, $80 \mathrm{~m} \times 40 \mathrm{~m}$, comparing solution accuracy improvement with an increase of refinement. FEFLOW has shown higher accuracy compared with other software for this generally occurring dispersivity values (range of values). 


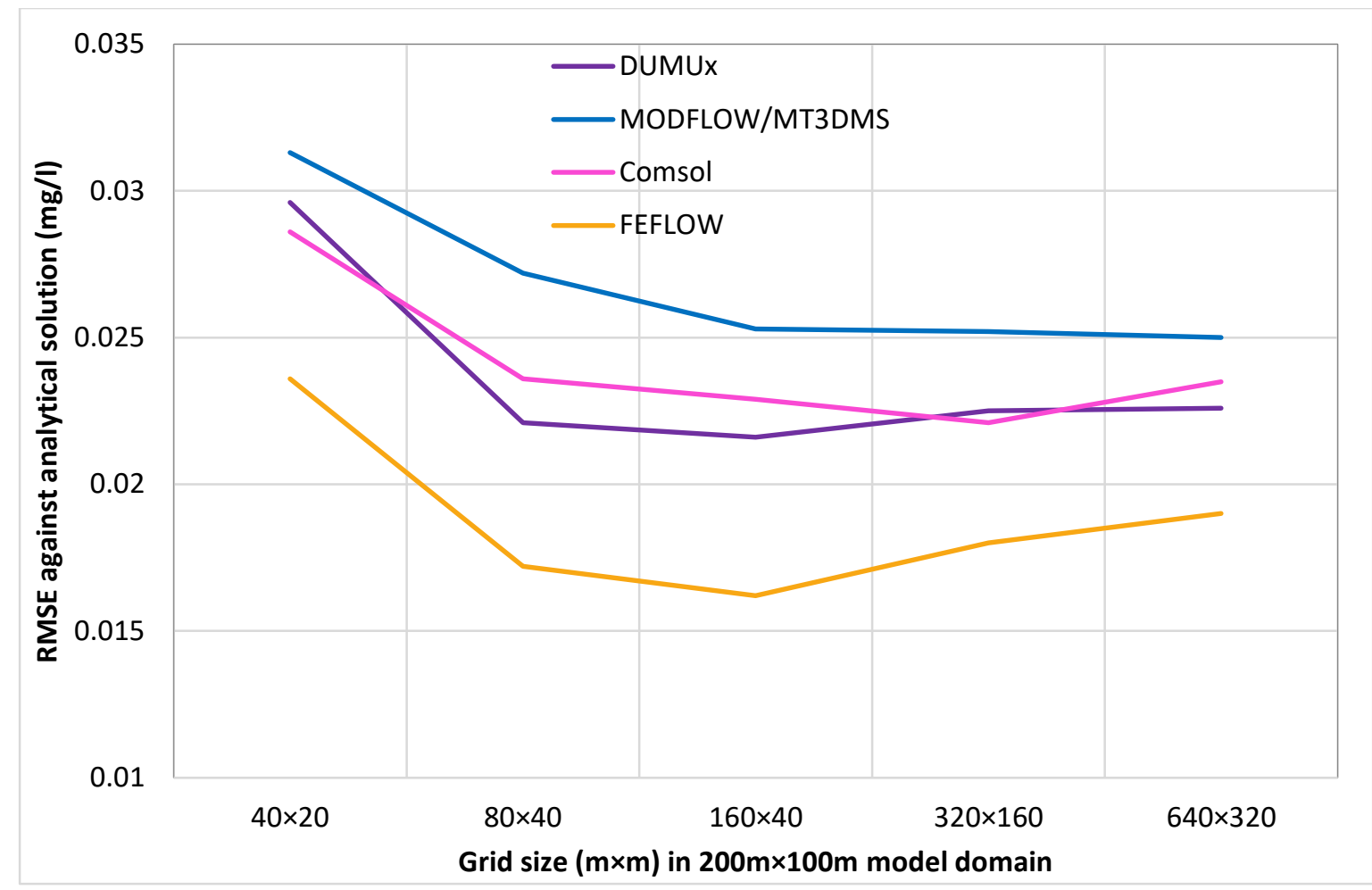

Figure 3.2.8: Spatial discretization sensitivity on the solution accuracy convergence in different simulators for a standard dispersivity $5 \mathrm{~m}$.

\subsection{Problem 3: 3D -Flow and solute transport simulation in a layered georeservoir}

Figure 3.2.9a-c shows the simulated breakthrough curves in the top, middle and lower aquifer of the reservoir, respectively. Since no analytical solution is available for such a complex case, the breakthrough curves were only compared to one another and solution accuracy could not be derived. From the Figs. 3.2.9a, 9b, and 9c, it is revealed that tracer breakthrough in the different layer varies significantly both in peak concentration and peak arrival time. Peak concentrations are highest in all layers for COMSOL. This is especially pronounced in the top aquifer (Fig. 9a). DuMu" shows a significantly stronger "smoothing", i.e. higher concentrations during the rising limb and the tail and lower peak concentration, compared to the other simulators. This effect is already seen in the 2D simulation (problem 2) and in the 1D simulation (problem 1) for low dispersivity. FEFLOW and MODFLOW range in the sharpness of the peak between COMSOL and DuMu ${ }^{\mathrm{x}}$. While MODFLOW shows significantly lower peak concentrations than COMSOL for all layers, FEFLOW shows similar concentrations to MODFLOW in the top layer (Fig. 9a), similar concentrations to COMSOL in the middle layer (Fig. 3.2.9b) and concentrations in between those two in the bottom layer (Fig. 3.2.9c). 

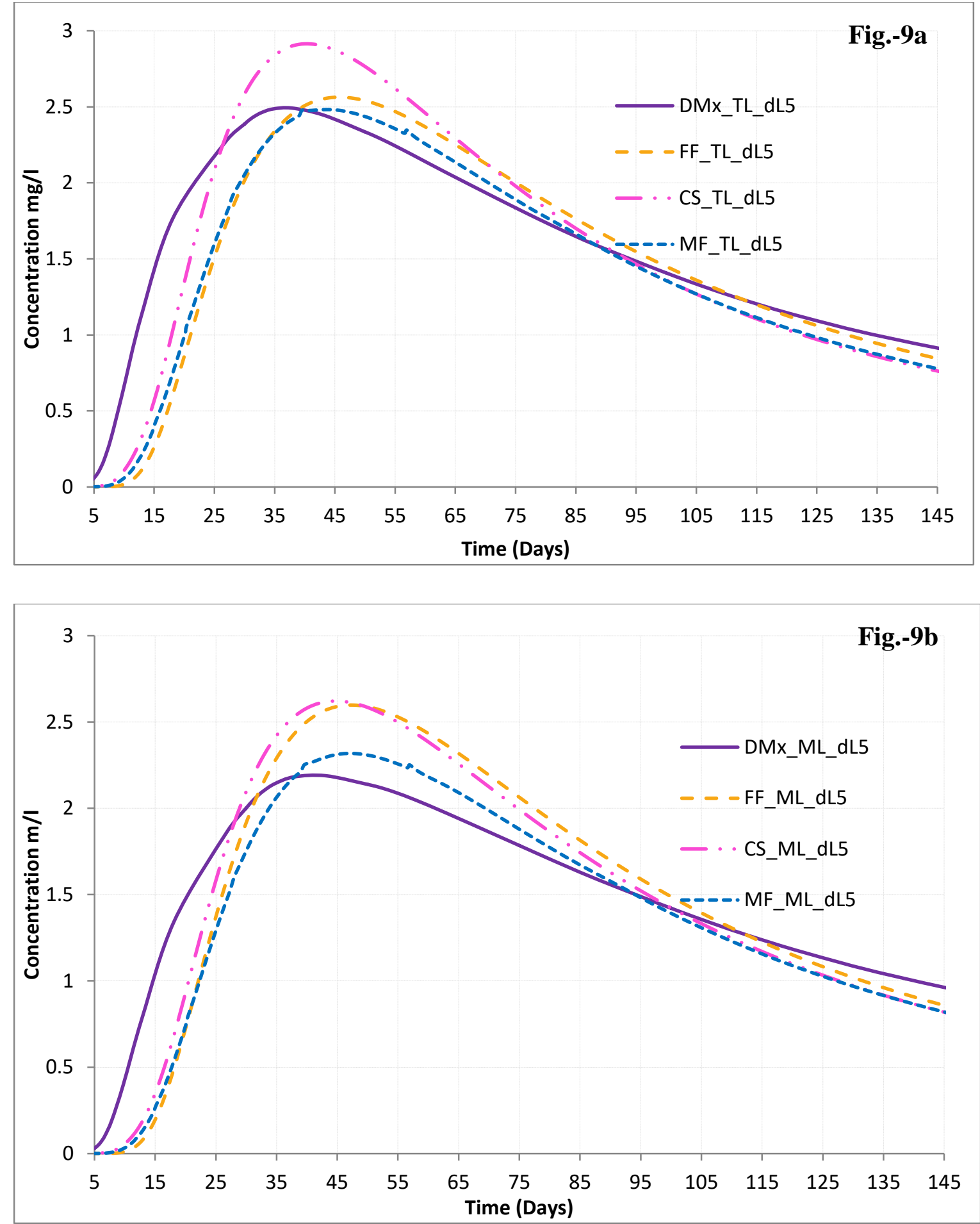


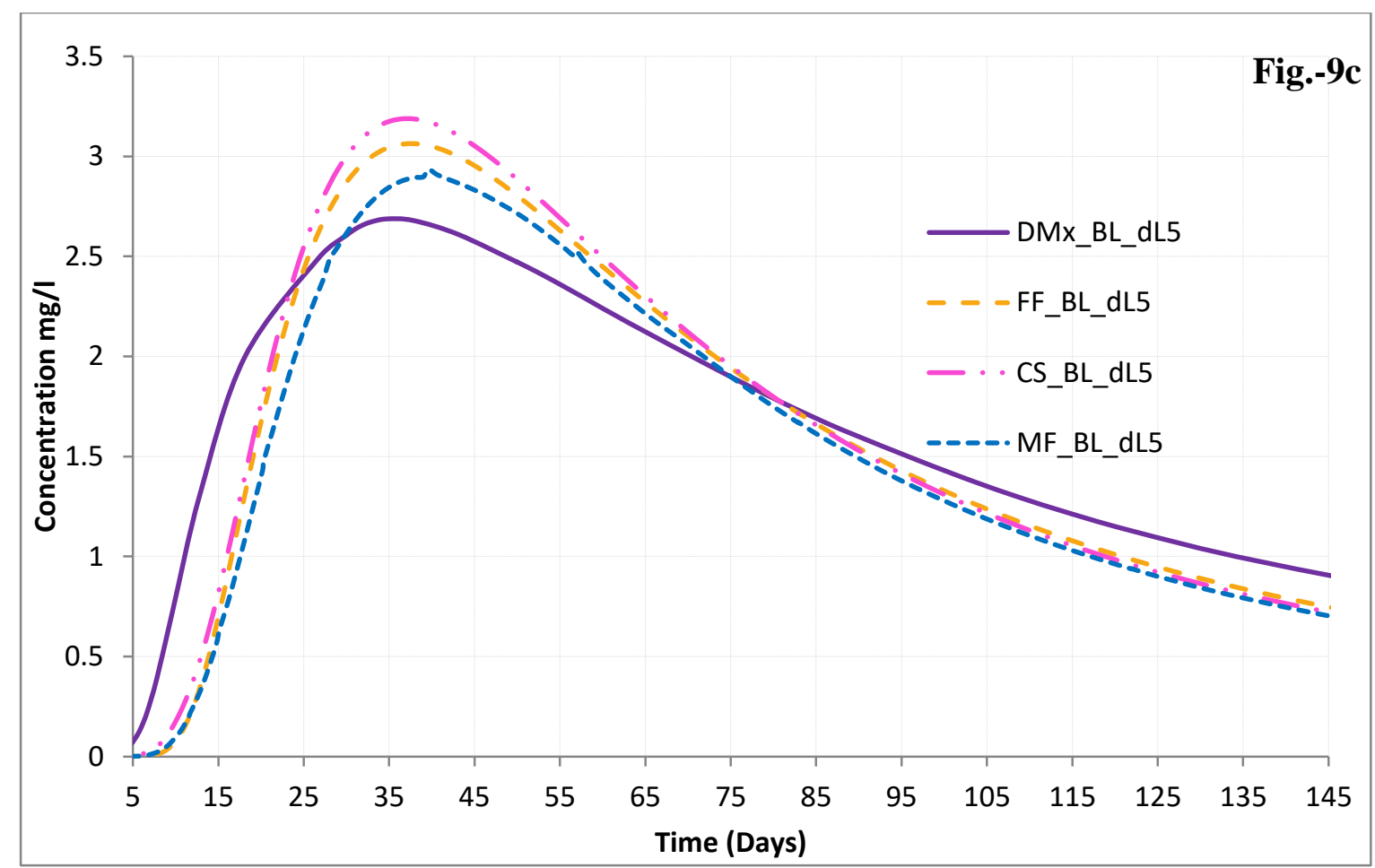

Figure 3.2.9a-c: 3D model tracer concentration from numerical simulation using MODFLOW, FEFLOW, COMSOL Multiphysics and DuMux respectively at three different layers a) top layer b) middle layer c) bottom layer with a dispersivity value $5 \mathrm{~m}$.

The relative difference between the tracer breakthrough curves is studied using MODFLOW/MT3DMS as a reference since it shows the largest similarities to the other codes with peak concentrations lying more or less in the middle between $\mathrm{DuMu}^{\mathrm{x}}$ and COMSOL. The RMSE with MODFLOW/MT3DMS for different dispersivities (Fig.3.2.10a-c) shows significantly higher differences than for the 1D and 2D cases. While errors in the lower dimensional cases (1D and 2D) range between 0.1 to $8 \%$, the differences in the 3D case range between ca. 0.1 and 0.7. As for the other simulations, dispersivity around $3 \mathrm{~m}$ achieves the "best" fit for COMSOL and FEFLOW. For DuMu the fit can be improved further if slightly higher dispersivity around $7 \mathrm{~m}$ is chosen. 

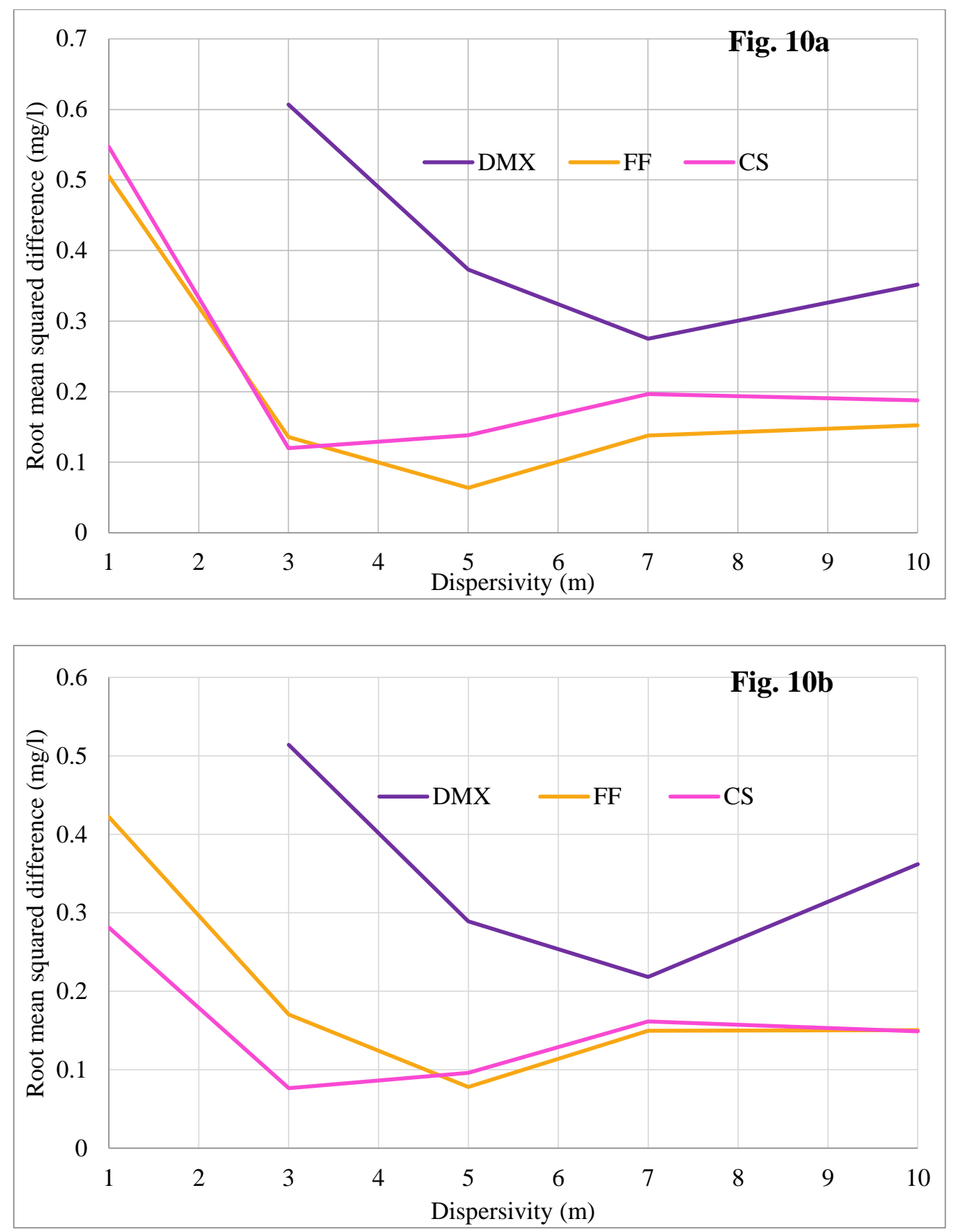


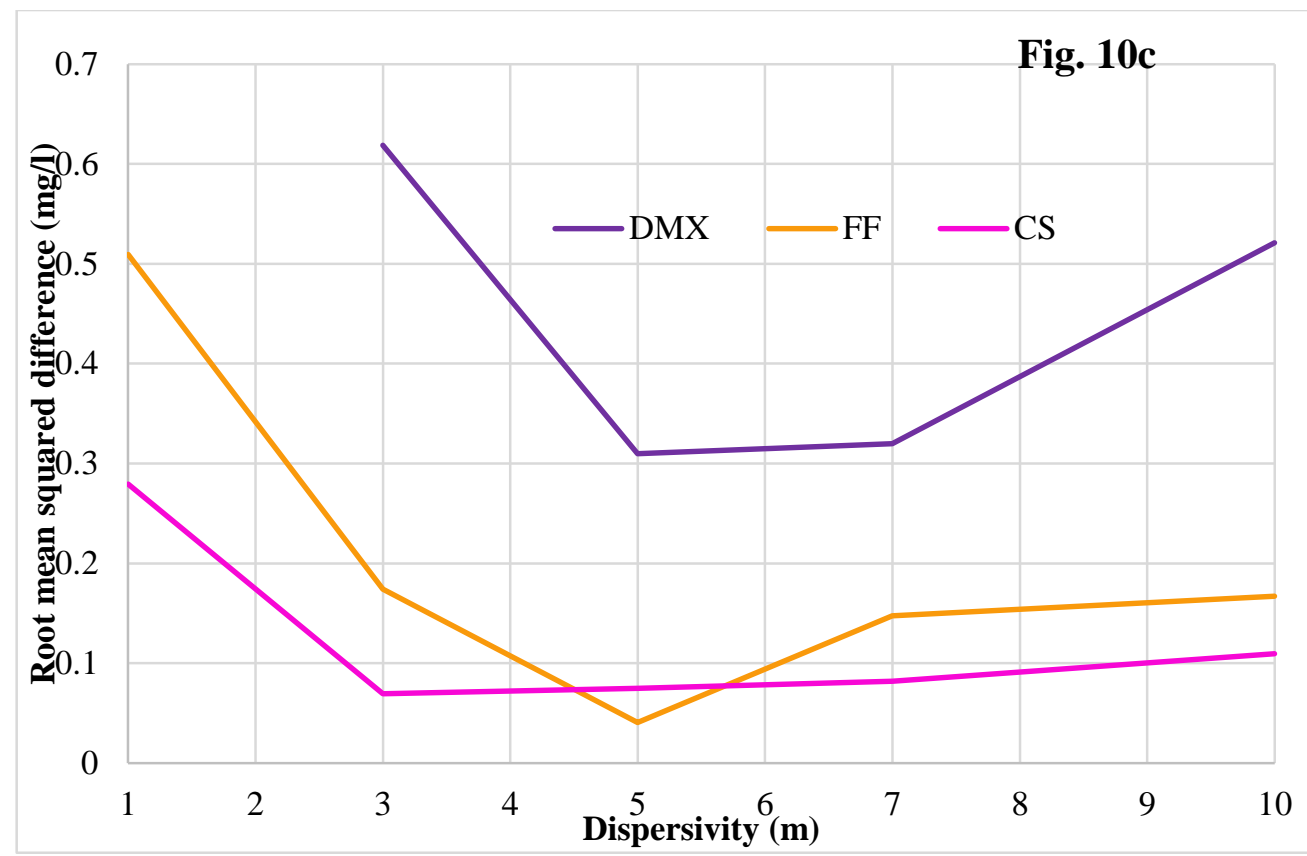

Figure 3.2.10a-c: Relative difference of tracer concentration for different dispersivity from concentration curve of MODFLOW/MT3DMS at three different layers a) top layer b) middle layer c) bottom layer.

\subsection{Simulation time (CPU time) of single processor and parallelization}

There are two ways to significantly shorten computing time by a numerical code to solve a specific problem. The first way is to improve and refine the computational methods for the model, for example, preconditioned conjugate gradient (PCG) methods and the link-algebraic multi-grid (LMG) package (Mehl and Hill 2001) linking MODFLOW to an algebraic multigrid (AMG) solver for matrix equations. Secondly, by using parallel computing methods to improve the computational performance of the numerical codes on supercomputers or multiprocessor (multi-core) systems. Recent parallel computer architectures provide both increased performance with respect to execution time and offer an amount of memory storage that significantly exceeds traditional single central processing unit (CPU) computers. Among the participating software, some independent developers have implemented parallelization in MODFLOW (e.g. Dong et al. 2013, Ji et al. 2014) and compared with old code. Whereas multithread computing is a default option and well implement in FEFLOW since 2008 and in COMSOL, it was a part from beginning. Flow simulation time in MODFLOW is relatively small compared with transport simulation time in MODFLOW/MT3DMS for the 2D and 3D cases (Problem 2 and 3). Hence, parallel computing time was not estimated for MODFLOW/MT3DMS as for transport simulation in MT3DMS parallelization was not 
reported yet. The computation time by the software packages was estimated for the defined problems in 2D and 3D. For both of the cases, automatic time step refinement is also implemented in the software. Though simulation period is 200 days with a time step size of 0.5 days (maximum time step size) the number of time steps used by the software are not identical. For example, in 3D simulation, time steps for COMSOL, FEFLOW and MT3DMS are 503, 402 and 409 respectively. Moreover, the time step needs to adjust with an increase of spatial discretization which is not in the same range for different software.

Table 3: Simulation time: Computational time in the simulation computer for the problem 22D domain and refined mesh.

\begin{tabular}{|c|cccc|cc|}
\hline \multirow{2}{*}{$\begin{array}{l}\text { Number of } \\
\text { Elements }\end{array}$} & \multicolumn{5}{|c|}{ Simulation Time (in Seconds) } \\
\cline { 2 - 7 } & \multicolumn{4}{|c}{} & $\begin{array}{c}\text { Computation in 4 cores } \\
\text { (Parallel computing) }\end{array}$ \\
\hline $100 \mathrm{~m} \times 200 \mathrm{~m}$ & COMSOL & FEFLOW & MODFLOW/MT3DMS & DuMux & COMSOL & FEFLOW \\
\hline $20 \times 40$ & 10 & 12.6 & 0.831 & 19.853 & 12 & 11.2 \\
$40 \times 80$ & 17 & 24.5 & 3.051 & 73.654 & 19 & 24.5 \\
$80 \times 160$ & 49 & 46.7 & 39.91 & 298.689 & 44 & 41.1 \\
$160 \times 320$ & 205 & 120.6 & 583.299 & 1267.602 & 175 & 92.9 \\
$320 \times 640$ & 977 & 517.6 & 9307.75 & 6893.497 & 802 & 369.2 \\
\hline
\end{tabular}

Table 4: Simulation time: Computational time in the simulation computer for the problem 33D domain and refined mesh.

\begin{tabular}{|c|cccc|cc|}
\hline \multirow{2}{*}{$\begin{array}{l}\text { Number of } \\
\text { Elements }\end{array}$} & \multicolumn{5}{|c|}{ Simulation Time (in Seconds) } \\
\cline { 2 - 8 } & \multicolumn{4}{|c}{} & \multicolumn{2}{c|}{$\begin{array}{l}\text { Computation in 4 cores } \\
\text { (Parallel computing) }\end{array}$} \\
\hline $50 \mathrm{~m} \times 100 \mathrm{~m} \times 12 \mathrm{~m}$ & COMSOL & FEFLOW & MODFLOW/MT3DMS & DuMu $^{\mathrm{x}}$ & FEFLOW & COMSOL \\
\hline $20 \times 40 \times 24$ & 1421 & 150 & 23.28 & 167859 & 83.2 & 1392 \\
$40 \times 80 \times 24$ & $* *$ & 616 & 151.47 & $*$ & 273.9 & 68400 \\
$80 \times 160 \times 24$ & $* *$ & 2306.6 & 2024.115 & $*$ & 1125.6 & $* *$ \\
$160 \times 320 \times 24$ & $* *$ & 12849.99 & 24038.01 & $*$ & 6331.8 & $* *$ \\
$320 \times 640 \times 24$ & $* *$ & 61880.00 & 570000.5 & $*$ & 50622.55 & $* *$ \\
\hline
\end{tabular}

*Grid creation failed, grid error, ** Out of Memory- Time step could not converge

Tables 3 and 4 reveal that simulation time is significantly reduced in the simulators FEFLOW and COMSOL with the implementation of parallel computing. For a smaller number of grid elements, MODFLOW/MT3DMS shows the highest efficiency to simulate the result. With a higher number of mesh elements, the transport simulation code MT3DMS computational time increases due to the reduction in time steps size as well as an increase in computational time for each time step. Overall, computational efficiency is higher for MODFLOW/MT3DMS and multicore computation efficiency or parallelization efficiency is higher in FEFLOW. Important 
to note that due to higher memory requirement for $\mathrm{COMSOL}$ and $\mathrm{DuMu}^{\mathrm{x}}$, with a higher number of mesh elements (>22000), it was impossible to compute the simulation time by our benchmark computer, as simulations did not compute.

\section{Discussion}

\subsection{User friendliness}

The first author was familiar with the use and implementation of flow and solute transport in FEFLOW; similarly, other authors are familiar/expert in other participating software. Instantly, the first author has experienced and learned all software within the frame of this work, set up the models, run the simulation and extracted data for further analysis. Therefore, the first author was in a position to comment about user friendliness for new users for three participating software, COMSOL, MODFLOW/MT3DMS and DuMu ${ }^{\mathrm{x}}$. Besides a number of M Sc. students working under the supervision of the authors have learned the software and has relates their experience. Eventually, sources of errors have identified, interpret the difference in the simulation result during follow-up discussions. Model builder GUI that is offered in COMSOL and FEFLOW are very useful and easily grasped by a new user to develop and implement a problem in a numerical platform. The main program window of COMSOL is well organized and particularly intuitive. The model setup is tailored by defining a series of PDEs to describe the simulated physical phenomena. All the components of the constructed model can be accessed and edited in a panel (Model Tree) on the left side of the screen program window. The COMSOL simulation environment facilitates all steps in the whole modeling process: defining geometry, specifying physics, meshing, solving, and then post processing. The same is true for FEFLOW. MODFLOW/MT3DMS is supported by various commercial GUI (e.g. Visual MODFLOW) or few freely available GUI, e.g. ModelMuse is relatively easy to grasp due to its feature-based boundary condition naming that pertains (e.g. evaporation, recharge, and river etc. type boundary conditions). DuMu ${ }^{\mathrm{x}}$ is not supported by any pre-processing GUI which is rather disadvantageous. On the other hand, it offers a higher control over the simulation process and parameter estimation through building problem script, modifying and improving it for the desired process or simulation. The GUIs in COMSOL and FEFLOW allow for easy modification of parameters, but the source code cannot be edited or viewed by the user, which reduces the control and insight on the simulation process. Moreover, FEFLOW and MODFLOW/MT3DMS rather boosted with strong documentation and case studies for flow and solute transport than COMSOL and DUMU' 


\subsection{Solute transport simulation efficiency of the benchmark problems}

The benchmark problem 1-1D related to the tracer transport from point source contaminants in a homogenous, isotopic condition with a natural flow gradient. The finite element based FEFLOW and COMSOL time-concentration curves are the closest to the analytical solution revealed by a low RMSE (Figure 7) for this problem. For the 1D problem case, the differences with these two simulators are significantly lower than $5 \%$ of $\mathrm{C}_{0}$ except for very small dispersivity value $(0.1 \mathrm{~m}-1 \mathrm{~m})$ and in 2D case, COMSOL and FEFLOW show lower than 2\% of $\mathrm{C}_{0}$. Exceptionally low dispersivity $(0.1 \mathrm{~m}$ to $0.7 \mathrm{~m})$ cases show higher relative errors from an analytical exact solution which might be associated with bigger mesh size compared to dispersivity values i.e. higher cell Peclet number related numerical dispersion. Problem 2 (2D) showed larger differences with lower dispersivity values (0.1-1 m). However, the difference is not significant for higher dispersivity values $(3 \mathrm{~m}-10 \mathrm{~m})$. On that instance, it is worth to mention that the time-concentration curves from different software packages are clearly dissimilar to each other. Moreover, the numerical error that is seen in relative simple 1D cases reveals that a plausible numerical solution only can be achieved for dispersion values ranging between $0.7 \mathrm{~m}-7 \mathrm{~m}$ or highly discretized, simple flow condition, close to a linear problem, which is sufficient enough to limit expectation for solute transport modeling using a numerical solution. So, a difference in the tracer breakthrough from different software for the different aquifer layers in problem $3(3 \mathrm{D})$ is well expected. However, the observed variations among the software are significant even if they are only compared for commonly perceived dispersion cases (c.f. Figs 10). The variation among the simulated peak arrival time should be associated with permeability and porosity assignment or the way the fluid injection is simulated in the software code. For 3D cases, it proves, although there surely are differences in the implementations of boundary condition as well as treating hydraulic parameters across the layers. However, it observes that all participating software are capable of accounting the relevant processes.

Boundary Condition implementation in the software: A common error can occur through introducing inappropriate boundary conditions. It is important to note that when a constant concentration boundary condition (Dirichlet BC) is imposed in a transport model for example in problem 1 1D and problem 3 3D, a solute flux will be forced into or out of that cell in order to maintain the specified value of concentration, and the flux can occur by both advection and dispersion processes (e.g., Konikow et al. 1997). In this case of study, we find that no well- 
bore conditions work better in the case of imposing multiple well. Moreover, constant concentration over time is unlikely regardless of changes in the accompanying flow field or in local concentration gradients from hydrogeological and geochemical conditions points of view. Thus, except perhaps to represent concentration in a large open body of water bounding an aquifer (head boundary or open flow boundary condition), or for a boundary far from an area containing a solute plume of interest, it would rarely be realistic or appropriate to apply a constant concentration boundary condition to a field problem (Konikow 2011). A higher amount of mass transfer is estimated in MODFLOW and FEFLOW ( $30 \%$ of the total fluid mass in problem 3-3D), whereas, COMSOL shows a minor influence from open flow boundary.

Hydraulic conductivity can vary greatly over short distances, and heterogeneity can exhibit large spatial correlations, persistence, and connectedness. Overall, the more accurately and precisely a simulation model represents the actual distribution of $\mathrm{K}$, the better it can simulate the "true" velocity distribution in space and time. Particularly benchmark problem 3 revealed that, once heterogeneities and anisotropy are introduced, the model predictions diverge. A reason for this is probably the different implementation of the spatial distribution of hydrogeological parameters, such as permeability and porosity, that make use of different spatial discretization methods among the software packages e.g. highly contrasting permeability layer was additionally discretized in $\mathrm{DuMu}^{\mathrm{x}}$, cell centered parameter distribution in MODFLOW/MT3DMS.

Major sources for deviating in implementation of model or simulation results are

- Gridding induced error.

- Boundary condition implementation and constraints in the software, for example, FEFLOW needs higher mesh density near injection/pumping points, and same is true for COMSOL.

- Limitation of certain software to implement specific boundary conditions, for example, COMSOL is unable to converge the flow simulation while 'no flow' boundaries are imposed everywhere but at the wells and in FEFLOW, head is not stable without a refined grid at the injection point. 


\subsection{Model implementation, simulation time and resource use efficiency and discretization}

The advantage of finite element based benchmarked software (i.e., FEFLOW and COMSOL) are not explored explicitly through generating flexible meshes for complex geometrical domains. The inability of using flexible triangular meshes is the major limitation to finite difference based code MODFLOW/MT3DMS.

All simulators, except FEFLOW, show stable pressure condition at the multi-layered injection and pumping well throughout the simulation period. FEFLOW pressure results at the injection and pumping well shows oscillations during early simulation period (simulation time 0-5 days) and achieve a steady flow condition before the tracer reaches the observation point. It is important to mention here that well or highly variable flow and transport boundary conditions require a higher mesh density to simulate a stable flow condition in FEFLOW. Hence, in 3D problem, the total number of mesh elements in FEFLOW was 192 elements higher than MODFLOW and COMSOL (19200 elements).

The vertex-centred finite volume method based $\mathrm{DuMu}^{\mathrm{x}}$ simulator requires at the first step the construction of the finite element mesh and the assignment of flow and transport properties (i.e., porosity and permeability) in the finite element nodes. The finite element nodes are then the centres of the control volumes in the secondary finite-volume mesh, constructed by uniting the bary-centre of the finite elements and the mid-points of the finite element edges (e.g., Helmig 1997). Therefore, imposing the finite element nodes at the interface separating two layers cannot represent correctly the properties. This problem can be solved by setting two finite element nodes equally distanced from the actual layer, each assigned with the individual property of the layer. For Problem 3 it resulted in 3200 additional elements in DuMux

Computational time and memory use: The participating software simulation time results (Table 1 and Table 2) revealed a strong difference in their numerical performance. For comparing the simulation times COMSOL, Dumu ${ }^{\mathrm{x}}$ and FEFLOW use the same rectangular grid as MODFLOW. Even with the same simulation code, there are numerous choices, such as restrictions on time steps or regularizations of constitutive relationships, which may improve accuracy at the cost of greater runtime.

COMSOL require a higher run-time memory (RAM) to run the simulation. Memory reliance from $\mathrm{DuMu}^{\mathrm{x}}$ is mostly required for grid creation (UG-Grid module was used for problem 3). Whereas, COMSOL requires a higher memory to store the solution for each time step during 
the simulation period. It is worth observing that, in a real-world case, the variability of the geological input data is likely to be (orders of magnitude) larger than the differences between the simulation codes. Therefore, while there is undoubtedly a need to test the limits of simulation codes and see that all the desired physics are included, if handled correctly, the contribution to the final uncertainty in simulator predictions from the code itself is likely to be relatively small.

\section{Conclusions}

In low dispersivity cases (e.g. dispersivity $=0.5 \mathrm{~m}$ or lower), numerical simulation results show significant oscillations or are not converging at all in finite element software packages FEFLOW and COMSOL. The finite difference code MODFLOW/MT3DMS is stable for very low or no dispersivity as well as the finite volume software $\mathrm{DuMu}^{\mathrm{x}}$. However, in all those cases of low dispersivity, relative errors with the analytical solution are significantly higher. This is especially true for $\mathrm{DuMu}^{\mathrm{x}}$, for which the good convergence comes at the cost of significant numerical dispersion. For 1D and 2D cases all three tested modeling software show good agreement with the analytical solutions. By refining the grid discretization all four software packages get an improvement in accuracy. It is shown for the 2D problem that COMSOL Multiphysics needs a finer mesh to produce the same accuracy as FEFLOW and DuMu ${ }^{\mathrm{x}}$. For the choice of the appropriate simulation software, the specific demands of the problem statement need to be considered. For transport simulations in forced gradient where a commonly expected dispersion or higher value case, the finite element software FEFLOW is the best choice. Due to the high requirements on mesh refinement, assembling the model in virtual memory, COMSOL Multiphysics has the highest demand on computer resources. The larger solution time of COMSOL can, however, is compensated by its very intuitive user's interface, which makes the implementation of different problems rather fast and does not need any changes in the source code. On the other hand, $\mathrm{DuMu}^{\mathrm{x}}$ is academic free open-source code - so there is a tradeoff between purchasing a commercial code which may be fast to learn but later lead to long times of simulations. For single-phase transport problems COMSOL is still a good choice, however for more complex physics (multi-phase flow etc.) COMSOL may prove to be less efficient.

\section{Acknowledgements}


The authors like to thank Dr. Iulia Ghergut for her valuable comments and suggestions. The first author thankfully acknowledges the financial support from DAAD to pursue his doctoral study.

\section{References}

Anderson, M.A., 1984. Movement of contaminants in groundwater: Groundwater transportAdvection and dispersion. In: Groundwater Contamination, National Academy Press, Washington, D.C.: 37-45.

Bastian, P., Blatt, M., Dedner, A., Engwer, C., Klöfkorn, R., Kornhuber, R., Ohlberger, M., Sander, O. 2008. A generic grid interface for adaptive and parallel scientific computing. Part I: abstract framework. Computing 82, 103-119.

Bear, J. 1979. Hydraulics of Groundwater, 569. New York: McGraw-Hill.

Class et al, 2009, A benchmark study on problems related to CO2 storage in geologic formations, Summary and discussion of the results Comput Geosci (2009) 13:409-434, DOI 10.1007/s10596-009-9146-x.

Dong Yanhui and Li, Guomin 2009, A Parallel PCG Solver for MODFLOW, GROUND WATER $47(6), 845-850$.

Diersch, H.-J., G., 2009. FEFLOW 5.4: finite element subsurface flow and transport simulation system. Reference manual. DHI-WASY Ltd., Berlin, Germany, 292 pp

Diersch H-J G., 2013, FEFLOW: Finite Element Modeling of Flow, Mass and Heat Transport in Porous media, Springer, DHI-WASY GmbH, Berlin, Germany, 996pp

Flemisch, B., Fritz, J., Helmig, R., Niessner, J., Wohlmuth, B.: DUMU ${ }^{\mathrm{X}}$ 2007: a multi-scale multiphysics toolbox for flow and transport processes in porous media. In: Ibrahimbegovic, A., Dias, F. (eds.) ECCOMAS Thematic Conference on Multiscale Computational Methods for Solids and Fluids, Cachan, 28-30 November 2007

Harbaugh, A.W., E.R. Banta, M.C. Hill, and M.G. McDonald. 2000. MODFLOW-2000, The U.S. Geological Survey modular ground-water model - user guide to modularization concepts and the ground-water flow process. U.S. Geological Survey Open-File Report 00-92, 121 pp.

Hill, M.C., 1998. Methods and Guidelines for Effective Model Calibration. U.S. Geol. Survey Water-Res. Invest. Rep. 98-4005: 90 pp.

Joodi, A. S., Sizaret, S., Binet, S., Bruand, A., Alberic, P., and Lepiller, M., 2010. Development of a Darcy-Brinkman model to simulate water flow and tracer transport in a heterogeneous karstic aquifer (Val d'Orléans, France). Hydrogeology Journal 18, 295-309, doi:10.1007/s10040009.0536-x.

Jin, Y., Holzbecher, E., and Sauter, M., 2014. A novel approach using arbitrary LagrangianEulerian (ALE) method for the flow simulation in unconfined aquifers. Computers and Geosciences 62, 88-94. 
Ji X, Li' D. Cheng' T. Wang, Xu-Sheng and Wang, Q., 2014, Parallelization of MODFLOW Using a GPU Library, Groundwater Vol 52 (4), 618-623

Mehl S. and Hill, M. C. 2001, A Comparison of Solute-Transport Solution Techniques and Their Effect on Sensitivity Analysis and Inverse Modeling Results, 39(2), 300-307. DOI: 10.1111/j.1745-6584.2001.tb02312.x

Oehlmann, S., Geyer, T., Licha, T., and Birk, S., 2013. Influence of aquifer heterogeneity on karst hydraulics and catchment delineation employing distributive modeling approaches. Hydrology and Earth System Sciences 17, 4729-4742, doi:10.5194/hess-17-4729-2013.

Oehlmann, S., Geyer, T., Licha, T., and Sauter, M., 2015. Reducing the ambiguity of karst aquifer models by pattern matching of flow and transport on catchment scale. Hydrology and Earth System Sciences 19, 893-912, doi:10.5194/hess-19-893-2015.

Ogata, A, and Banks, R. B. 1961, A solution of the differential equation of longitudinal dispersion in porous media." Profl. Paper No. 411- A, U.S. Geological Survey, Washington, D.C.

Qi Li, Kazumasa Ito, and Zhishen Wu, Christopher S. Lowry, and Steven P. Loheide COMSOL Multiphysics: A Novel Approach to Ground Water Modeling GROUND WATER 47 (4).

Gelhar LW and Collins MA, 1971. General Analysis of Longitudinal Dispersion in Nonuniform flow. Water Resour. Res. 7, 1511-1521.

Konikow, L.F. 2011, The Secret to Successful Solute-Transport Modeling, Ground Water 49(2), $144-159$.

Konikow, L.F., W.E. Sanford, and P.J. Campbell. 1997. Constant-concentration boundary condition: Lessons from the HYDROCOIN variable-density benchmark problem. Water Resource Research 33(10), 2253-5561.

Zheng C and Wang P. P., 1999, MT3DMS A modular three-dimensional multispecies transport model for simulation of advection, dispersion and chemical reactions of contaminants in groundwater systems, U.S. Army Corps of Engineers Washington, DC, pp 239. 
CHAPTER 4: GENERAL DISCUSSION, CONCLUSIONS, AND FUTURE WORK 


\section{Discussion and conclusion}

Subsurface flow and transport parameter of estimation is the fundamental need to understand pathway and process, towards effective monitoring and management of groundwater aquifer, georeservoir of oil and gas, geological storage and geothermal reservoir. As a significant stake of future mix of renewable energy supply, the Enhanced Geothermal Systems (EGS) (Tester et al., 2006) substantially depending on the proper characterization of stimulated fracture. Interwell or single-well test give an opportunity for in-situ appraisal of the subsurface process. And many instance tracer methods can supplement geophysical method too, such as tracer based flow contribution estimation from multi frac-reservoir (Ghergut et al., 2016), which potentially can replace expensive individual fracture inflow profiling.

\subsection{Single well tracer injection-flowback/withdrawal test-early-time tracer signal study}

Sorptive tracer in stimulated georesevoir application: Colloidal nanocrystals ("quantum dots") can be made to fluoresce over a wide range, entirely covering the visible and near infrared (NIR) region of light - regions where geothermal and EGS reservoir waters possess very little interference (Rose 2011). Positively charged alkali and alkaline-earth elements (first and second rows of the Periodic table) can be used as matrix sorptive tracer (MST) for fracture porosity estimation (Karmakar et al 2015a, 2015b) as they found sorptive to granitic formations (Rose et al 2011). This suggests that positively charged ligands attached to the surface of quantum dot tracers would allow for their reversible sorption. Rose et al (2011) also reported that scale inhibitors such as polycarboxylates, polyacrylates, and polymaleic anhydrides sorb strongly but still reversibly within geothermal wellbores and formations. If these species are bonded to the quantum dots, they would render them reversibly sorptive on EGS formations, while retaining all of the fluorescence properties available from quantum dot tracers. Further possibility from this kind of quantum dot tracer revealed the use of relatively inert thin quartzgel coating to cover the quantum dot tracer. This concept has been successfully applied in bio-

imaging applications to ensure that cadmium and lead components cannot leak into the biological system (Bruchez et al, 1998; Rogach et al, 2000). Besides deepwell environmental protection, the quartz-gel coating is also ideally suited to reversely protect the nanocrystals from the high temperatures and potentially corrosive conditions in EGS tracing. Furthermore, since the silica layer is completely transparent to visible and NIR light, the emission-based tracing ability by the nanocrystals will not be affected. 
The research results from Rose et al (2011) will be particularly striking toward synthesis of water-soluble colloidal nanocrystals with single-wavelength excitable emission colors spanning the entire visible and NIR range and nanocrystal tracers of temperature and corrosionstable for deep-well applications by coating them with a thin layer of transparent silica. Rates of tracer diffusion out of fractures into secondary porosity (and back again) are dependent on tracer diffusivity, matrix porosity, and interfacial area between the fractures and matrix. By systematically varying tracer diffusivities, interfacial area can be estimated from differences in tracer responses, provided reasonable estimates of matrix porosity exist (Rose et al. 2011). Though, it is evident after Karmakar et al (2015a, and 2015b) that diffusivity based parameter estimation will be less or unattractive for the application of "early time tracer flowback test" scheme toward fracture characterization. Furthermore, the use of halides as tracers is generally limited to bromide and iodide, and because of their large diffusivities, the background concentrations of these tracers in EGS reservoirs will rapidly increase to the point where they can no longer serve as effective tracers (they could only be used once or twice). However, if quantum dot tracers with different optical signals were available, they could be used repeatedly without background interferences along with many existing non-interfering solute tracers that have larger diffusivities than halides (e.g., the naphthalene sulfonates) to conduct repeated interrogations of surface area as an EGS reservoir is developed and stimulated (Rose et al. 2011).

The responses of the halide and naphthalene sulfonate represent the current state of the art in differences in breakthrough curves that can be obtained due to different diffusivities of geothermal tracers. Quantum dot tracers with controllable surface sorption characteristics also have the potential to provide surface area estimates in geothermal reservoirs. Such tracers could prove especially useful when the reservoir matrix porosity is so low that it is difficult to observe a contrast in breakthrough curves of tracers with different diffusivities or using very short duration test for early time tracer signal. Rose et al (2011) also showed in their hypothetical example of sorbing tracers with well characterized sorption properties (sorption and desorption rates and sorption capacities as a function of surface area) that fracture surface area can be estimated in the absence of matrix diffusion. They also revealed that conservative tracers with different diffusivities will have identical breakthrough curves when reservoir matrix porosity is negligible, but the responses of sorbing tracers relative to conservative tracers can still provide estimates of fracture surface area. The combined use of diffusing and sorbing tracers in reservoirs with finite matrix porosity would, in principle, allow the effects of diffusion and 
sorption to be de-convoluted, which would reduce uncertainties in surface area estimates below that which can be achieved using diffusing or sorbing tracers alone. Moreover, the novel single well tracer test design of 'early-time' scheme will be particularly useful to discount diffusion effect on tracer flowback signal.

Dispersion controls the apparent 'arrival time' in thin WF-type fractures (Ghergut et al., 2013), which is strong enough for conservative tracers to enable dispersivity and aperture determination independently of each other. For thicker, GPF-type fractures, a stronger parameter interplay is found between dispersivity, fracture thickness and proppant-packing porosity. However, a rough estimation of dispersivity from conservative tracer signals is still possible since dispersivity effects are stronger than those of GPF thickness and proppantpacking porosity. Once dispersivity is identified (by this or some different approach as suggested in (Chapter 2, part 1, section 5), GPF-type fracture thickness and proppant-packing porosity can be determined independently of each other, assuming a suitable MST and PST data are available. The use of an MST enables thickness determination; and a PST allows proppant-packing porosity determination, if the thickness is not too small (>2mm). For WFtype fractures aperture determination is possible with conservative tracers alone, we found that the use of MST improves sensitivity. On that instance, complete fracture characterization for GPF-type fractures only becomes possible with MSTs and PSTs and fracture aperture for WF with conservative tracer while it assumed a rough dispersion estimation possible beforehand of the test.

Table 5: Early time tracer push-pull test uses in fracture characterization

\begin{tabular}{|l|l|l|}
\hline \multirow{2}{*}{ Early pull signals } & Short injection pulse & (Conservative solute tracer) \\
\cline { 2 - 3 } & $\begin{array}{l}\text { The peak arrival time can determine } \\
\text { the dispersivity in thin single fracture } \\
\text { (Water fracture) } \\
\text { Different slope with arrival time } \\
\text { determine fracture aperture }\end{array}$ & $\begin{array}{l}\text { Ambiguity on fracture thickness, } \\
\text { fracture porosity } \\
\text { Parameter estimation possible using } \\
\text { different sorptive tracer } \\
\text { Sensitive to fracture length in HDR } \\
\text { type EGS }\end{array}$ \\
\hline
\end{tabular}

Solute diffusivity, assuming as a fracture aperture and spacing sensitivity parameter, has studied using a similar model concept similar to PST and MST. However, the use of conservative tracer pairs with different diffusivity, simulations have shown that matrix diffusion effect is far too weak to enable parameter inversion from early flowback signals. 
Alternatively, heat could be used as a 'matrix-diffusive tracer' instead of solute tracers, owing to heat diffusivities being much higher than solute diffusivities (Kocabas and Horne, 1987; Pruess and Doughty 2010; Jung and Pruess, 2011; Ghergut et al. 2011). However, during early flowback following multiple injectate slugs with different temperatures, it may be difficult to use "heat" for determining fracture parameters. The tracer-based procedure described in the present paper is also applicable in such cases; tracers should be added shortly before the end of the last injection cycle. This adaptability will be particularly useful to deploy this type of tracer test in very different stage fracture stimulation or monitoring.

Figures. 2.3.2 and 2.3.5 suggest that for the fracture length estimation from matrix sorptive tracer, it is essential to evaluate the tracer signal at very early stage. The 'early-middle' or 'early-late time' tracer signals would be insensitive to the fracture length for low sorptive tracer $(\mathrm{k}-0.7 \varphi \mathrm{m}=1 \%)$ as well as high sorption tracer $(\mathrm{k}-1.5 \varphi \mathrm{m}=1 \%)$. From the simulation results, in HDR type EGS geo-reservoir, tracer application scheme is recommended to evaluate fracture length as follows- a) Weak matrix sorptive tracers for relatively longer fracture length/higher fracture volume. The matrix sorption coefficient $\kappa$ value should be a range of 0.1 to 1 for a matrix porosity $0.5 \%-3 \%$. b) Strong matrix sorptive tracer would be recommended for all cases however specifically useful while stimulated fracture length expected to be relatively small $(<200 \mathrm{~m})$. c) Early-time tracer signal from conservative solute can determine the aperture of fractures created by a WF treatment. However, the effectiveness needs to be verified for very thin fractures $(<0.2 \mathrm{~mm})$ as suggested in (Karmakar et al., 2015a and 2015b).

\subsection{Tracer selection for early time tracer injection-flowback test}

To identify the minimum sorptivity (minimum $\kappa$ value) required to induce sufficient contrast between measurable tracer signals for different values of target parameters, the scheme to follow is: a $\mathrm{p} \%$ change in a target parameter value produces $\mathrm{c} \%$ change in the signal of a conservative tracer, and a $\mathrm{s} \%$ change in the signal of a sorptive tracer, $\mathrm{s}$ being a function of $\kappa$. One then needs to find out the minimum value of $\kappa$ that renders s significantly higher than $c$. This value cannot be prescribed in a universal manner because the very meaning of 'significantly higher' depends on how tracers' signals can be sampled and measured, a process always affected by various errors. In any case, the higher the value of $\kappa$, the higher is the sensitivity improvement. A lower sorptive tracer than its minimum necessary is suggested for PSTs or MSTs, assuming a sensitivity improvement factor (ratio between sorptive tracer signal changes to conservative tracer signals changes, s/c) approximately equal to $\sqrt{ }(1+0.7 \times$ sorption 
coefficient, $\kappa)$. This empirical relationship delimits that too much investment on highly sorptive tracer which ultimately will not affect tracer parameter sensitivity at same magnitude (Figure 4.1). One needs to note that the higher the tracer's retardation, the lower is its fracture invasion, and consequently a poorer capability for characterizing the fracture as a whole. In principle, this could be compensated by increasing the chaser volume (i.e., by injecting sorptive tracers earlier than conservative tracers) or longer injection duration rather applying higher the injection rate. Hence, for early-time tracer injection-flowback test, it is advisable to use a medium ranged MSTs $(0.7<\mathrm{k}<2.5$, matrix porosity 3\%) and PSTs $(25<\mathrm{k}<80)$, to avoid ambiguity due to the error in detection (Table 1).

The injection and flowback of a small fluid volume, and thus little dilution of the injected tracers, has three practical advantages:

(1) there is no need to inject large tracer quantities;

(2) one does not have to wait for the tails of the test signals; and

(3) the field and laboratory monitoring of the tracer signals do not have to be conducted for ultra-low tracer concentrations

Early time tracer signal based single well injection flowback test showed an improvement of SWIW method. This pulse injection and flowback based method have shown that if the flowback pressure builds up is sufficiently enough to simulate a flowback parameter determination from tracer signal is evident with a small number of sampling both in sedimentary formation or crystalline formation. This approach and method would eventually reduce the time and cost of long time sampling, waste water treatment. In some instance, the 'late-time' sampling as indicated by Ghergut at al. (2013) can be conducted to confirm the fracture aperture. For a late-time study, this small tracer remaining would be useful to confer a sensitivity regime rather repeated use tracer test for reservoir monitoring.

\subsection{Benchmark study for efficient numerical method selection and code development}

Benchmark study is crucial for numerical simulation correctness and predictability. Solutes transport in fracture still not a common to flow and solute transport software packages of porous media, unlike FEFLOW. Though intuitive PDEs interface is available in COMSOL, formulating comparable solute transport model in the fracture is not straightforward and 
currently failed to comprehend a similar result. It would be an interesting to implement fracture model using COMSOL due to various adaptive features in PDEs.

A set of benchmark studies that conducted in four different software with a gradual increase dimension for flow and solute transport shows that in reasonable dispersion ranges, a course mesh can simulate reasonably accurate result for $1 \mathrm{D}$ and $2 \mathrm{D}$ cases overall. For $3 \mathrm{D}$ cases, it proves, although there surely are differences in the implementations of the participating codes, that available models are capable of accounting for the relevant processes accepting a significant nonconformity with tracer arrival time and BT concentration among each other. Major sources for deviating results are by the gridding, boundary condition implementation and constraints in the software. For transport simulations in forced gradient where a commonly expected dispersion or higher value case, the finite element software FEFLOW is the best choice. The larger solution time of COMSOL can, however, is compensated by its very intuitive user's interface, which makes the implementation of different problems rather fast and does not need any changes in the source code, as is the case for DuMux. The participating software simulation time results revealed a sharp difference in their numerical performance. Nevertheless, the study showed that improving the numerical performance by optimizing discretization methods, solvers, and parallelization methods remain a vital field of research.

\section{A way forward}

\subsection{Early time solute tracer push-pull test for dispersion estimation in aquifer}

The study by Schroth et al. (2001) on the in-situ evaluation of solute retardation using SW push-pull indicates that in a homogeneous single-porosity medium, tracer 'pull' signals depend on porosity, thickness, and longitudinal dispersivity through the following product

(porosity $\times$ thickness $) \times($ dispersivity squared $)$, or

(effective aperture $) \times($ dispersivity squared $)$ 
It, therefore, follows that these three parameters cannot be determined independently from each other. Dispersivity has a stronger influence than porosity or thickness - a fact that can become useful. In groundwater remediation application considered by Schroth et al. (2001), this hydrogeological parameter interplay was not an issue, since the aquifer thickness and porosity are known in advance of conducting the SW tracer push-pull tests, and the unknown parameters were aquifer dispersivity and solute retardation.

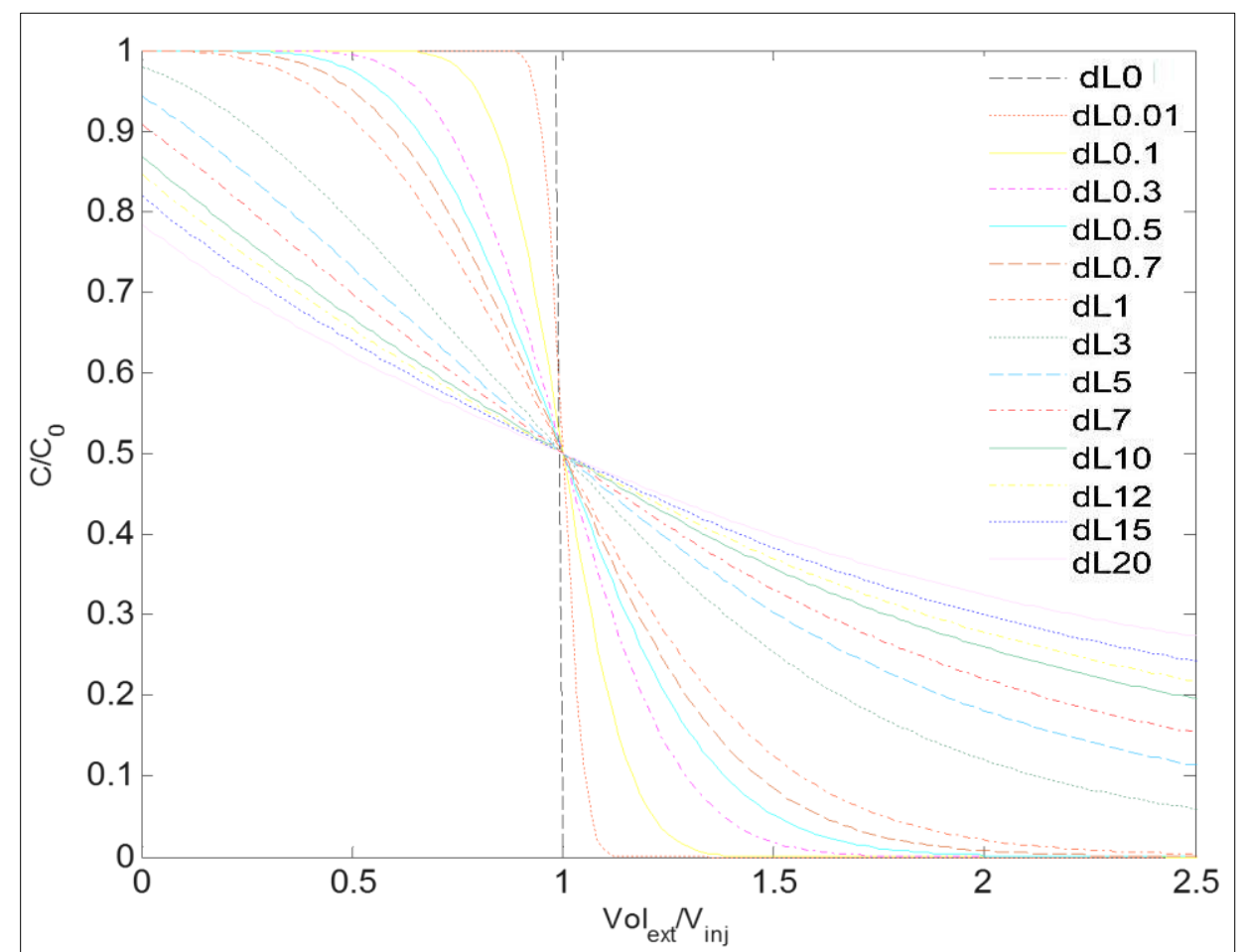

Figure 4.1: Dispersion value sensitivity of Schroth et al. (2001) single-well tracer push-pull test pull signal.

The higher dispersivity value assumed as a monotonically related with dispersion similar to sorption. In that formulation it would fail to describe higher dispersion value while ' $r$ _max' and subsequently dispersivity $\left(\alpha_{\mathrm{L}}\right)$ value at Schroth et al. (2001) equation (10-11) is would be idealized with an average value assuming a same value over the whole domain. However, while r_max is higher or tracer covers a longer radial distance during push phase and tracer would experience variable flowpath or higher dispersivity. In that instance, the assumption from Schroth et al (2001) no longer valid which also mentioned in Karmakar et al (2015a).

\subsection{Heat tracer uses for pulse injection flowback test-design and parameter estimation}

Initially early time tracer based study has convinced that tracer diffusivity would be a sensative parameter for fracture aperture, though, it is reported as independent with fracture apeture by 
Ghergut et al. (2013). The small test duration in early time test and zero contamination potentioal hence no treatment would be an ideal tracer for any tracer test. Solute tracer contribution for 'early-time' tracer injection-flowback test (Karmakar et al. 2015a, 2015b) has revealed the potential use of reliable diffusive tracer such as heat tracer uses in the similar test condition. It also believes to a characteristics flowback signal exist from heat diffusion in georeservoir especially from fracture spacing as discussed in Ghergut at al (2013). Due to very short test duration, heat diffusivity, (two order of magnitude higher diffusivity assumed) can be employ to determine single parameter while larger parameter ambiguity believed, mostly related with effect from matrix volume. Hence, the fracture spacing or fracture length would be a sensitive parameter to determine from this kind of test. One need to note that heat as a highly diffusive tracer, the effective diffusion coefficient $D$ differs between heat and solutes (in deep geothermal reservoirs) by at least three orders of magnitude. Test regime that is 'early' for solute tracers may already be 'very late' for temperature signals, and what is 'late' for temperature signals may still be 'early' for solute tracers (Ghergut et al 2013), depending upon the value of fracture spacing $a$, which 's hard to know in advance when dimensioning an SWIW test (Ghergut et al. 2011). Understanding the single well tracer flowback/pull signal from multiple wing-cracked HDR type georeservoir systems with a known number of stimulated fractures, diffusive tracer (heat tracer) pull signal with early-mid to mid-time duration can estimate fracture surface area as well can employ for fracture spacing. The combination of heat and solute tracers use in single-well early-time test can characterize a fracture length in HDR type EGS, where heat tracer can be employ for fracture spacing.

\subsection{Solute tracer pulses injection flowback test-design and parameter estimation in shale/gas} reservoir characterization

Last but not the least, flowback chemical tracer test, a standard practice in shale- petroleum geo-reservoir for residual oil saturation, early time tracer flowback test would be beneficial to implement for multiple parallel fracture parameter estimation. Moreover, as most of this study considered only homogeneous formation, it would be interesting to observe the effect of heterogeneity and multiphase condition on breakthrough tracer signal- few field scale study could be appropriate on this envisage.

Unconventional oil and gas reservoirs of low-permeability are emerging as an important source of energy in the United States and Canada (Frantz et al. 2005). Unconventional oil and gas reservoirs are commonly developed using multi-fractured horizontal wells (Ning et al. 1993) 
followed a form of injection of fracturing fluid (which is mainly consisting of water) during fracturing operations, is injected into the well to induce fracture networks (Engelder et al., 2014). The well is then shut-in (soaking period) to improve the hydrocarbon production (King, 2012; Lan et al., 2014-a; Makhanov et al., 2014) and later on allow or start put on flowback to recover the injected fracturing fluid (Abbasi et al., 2014). It is shown from field data that water initially injected into the well has vastly different chemistry than the water recovered during flowback (Rimassa et al., 2009; Zolfaghari et al., 2015).

Despite the economic justification of hydraulic fracturing, its adverse environmental impacts have raised serious concerns about the contamination of ground and surface water as well as the consequential hazards to the environment and public health (Jackson et al., 2013; Vengosh, 2014). Knowledge of flowback water composition and the origin of flowback ions are required for water environmental assessment and selection of appropriate remediation strategies. Moreover, analysis of flowback chemical data has provided a new approach for quick characterization of the induced fracture network (Gdanski, 2010, Ghanbari et al. 2013, Bearinger 2013). A qualitative correlation was developed between fracture network complexity and salt concentration profiles measured during the flowback period by Bearinger (2013), Ghanbari et al. (2013) and Ghanbari and Dehghanpour (2016). The source of ions in flowback water is still a matter of debate, though assessing chemical analysis during fracturing operations widely in practice. Among many reasons, dissolution of rock constituents (Blaunch et al. 2009), mixing of the injected frack fluid with in-situ formation brine (Haluszczak et al. 2013) ion exchange reactions with clay minerals from shale (Carman and Lant, 2010) have identified as a primary contributor of ion during flowback. Furthermore, mineral filled natural fractures and locally precipitated salts (Gale et al., 2014; Zolfaghari et al., 2014) can react with water and impact the flowback water chemistry during the hydraulic fracturing operations. Overall, understanding the source of produced ions and the factors controlling the flowback water chemistry is essential for interpreting the flowback.

Many current researches (Bearinger 2013, Ghanbari et al. 2013, Ghanbari and Dehghanpour 2015, Zolfaghari et al., 2015) have identified a connection with salt concentration profile during flowback with hydraulic fracture network. An early-time tracer injection flowback would be promising to investigate this aspect. Early-time tracer injection-flowback test has several possibilities to use for fracture characterization in unconventional oil-gas formation characterization ranging from fracture length, fracture thickness characterization using MSTs of suitable range. Moreover, proppant sorptive tracer (PSTs) can determine either fracture 
porosity or fracture thickness while hydraulic fracture in unconventional shale-gas formation contains infill to stabilize the fracture. Finally, reactive tracer uses can particularly helpful to identify in-situ reaction, fate and transport of contaminant either using early-mid time flowback signal which can be adjustable with the shut-in period (King, 2012; Lan et al., 2014-a; Makhanov et al., 2014) that apply to improve the hydrocarbon production. On this instance of source identification chemicals during flowback, a single-well injection-flowback tracer test with competing ion of 'no' or 'very negligible' environmental impact can characterize pathway of specific chemicals eventually help to formulate a remediation mechanism.

\section{Final remark}

Early tracer flowback signal would be extremely useful for many EGS application, where reservoir characterization is an issue for its sustainability and management. Important to note that, interpreting a tracer test result from a stimulated georeservoir need to account many issues including thermos-geo-chemical behavior of intended tracer as well sampling, preservation of sample and analytical procedure. The error may arise from this uncertainty and complexity may dampen the 'signal' to a noise. Hence, Behrens (2009) expressed this as "playing with tracers" using SWPP test could never be an "uninteresting routine". Early time tracer push-pull signal based study for contaminated aquifer would open up a new approach to parameter estimation as it has observed in the simulation study for fracture georeservoir. This application is rather novel regarding the higher predictability of parameters, lower cost, and small environmental footprint, from a very short tracer test campaign. However, it is also anticipated to improve and develop its design for various field situation and interest. 


\section{References}

Abbasi, M.A., Ezulike, D.O., Dehghanpour, H., Hawkes, R.V., 2014, Journal of Natural Gas Science and Engineering, 17, 82-93.

Alivisatos, A.P. 1996, Semiconductor Clusters, Nanocrystals, and Quantum Dots, Science 271, 933-937.

Bearinger, D., 2013, Message in a bottle, SPE 168891, Unconventional Resources Technology Conference, Denver, Colorado, USA, 12-14 August 2013.

Behrens H, Ghergut I, Sauter M. and Licha T. 2009, Tracer properties, and spiking results - from geothermal reservoirs, Proceedings, 34th Work- shop on Geothermal Reservoir Engineering, Stanford University, Stanford, CA, SGP-TR-187.

Blaunch, M.E., Myers, R.R., Moore, T.R., Lipiniski, B.A., Houston, N.A., 2009, Marcellus shale post- frac flowback waters-where is all the salt coming from and what are the implications?, SPE 125740, SPE Eastern Regional Meeting, Charleston, West Virginia, USA 2009.

Carman, P.S., Lant, K.S., 2010, Making the Case for Shale Clay Stabilization, SPE 139030, SPE Eastern Regional Meeting, Morgantown, West Virginia, USA, 12-14 October 2010.

Dean, C., Reimus, P., Oates, J., Rose, P., Newell, D., Petty, S., 2015. Laboratory experiments to characterize cation-exchanging tracer behavior for fracture surface area estimation at Newberry Crater, OR. Geothermics 53, 213-224.

Efros, A.L., Lockwood, D.J., Tsybeskov, L., (Eds) 2003, Semiconductor Nanocrystals: From Basic Principles to Applications, Springer: New York.

Engelder, T. Cathles, L M. L., Bryndzia, T. 2014, The fate of residual treatment water in shale gas, Journal of Unconventional Oil and Gas Resources 7, 33-48

Frantz, J.H., Jochen, J.V., Shale Gas, Schlumberger White Paper, 2005. Gale, J. F. W., Laubach, S. E., Olson, J. E., Eichhubl, P., and Fall, A., 2014, Natural Fractures in Shale: A Review and New Observations, AAPG Bulletin, v. 98, no. 11, p. 2165-2216. doi: 10.1306/08121413151.

Gale, J. F. W., Laubach, S. E., Olson, J. E., Eichhubl, P., and Fall, A., 2014, Natural Fractures in Shale: A Review and New Observations, AAPG Bulletin, 98 (11), p. 2165-2216. doi: 10.1306/08121413151.

Gdanski, R., 2010, Numerical Study of PDL-induced fracture-face damage using a fracture mimicator, SPE 132918, SPE Asia Pacific Oil \& Gas Conference and Exhibition, Brisbane, Queensland, Australia, 18-20 October 2010.

Ghanbari, E., Abbasi, M.A., Dehghanpour, H., Bearinger, D., 2013, Flowback Volumetric and Chemical Analysis for Evaluating Load Recovery and its Impact on Early-time Production, SPE 167165, SPE Unconventional Resources Conference-Canada, Calgary, Alberta, Canada, 5-7 November 2013.

Ghanbari, E., Dehghanpour, H., 2016, The fate of fracturing water: A field and simulation study, Fuel, 163,282-294. 
Ghergut, J., Behrens, H., Sauter, M., 2016, Petrothermal and aquifer-based EGS in the NorthernGerman Sedimentary Basin, investigated by conservative tracers during single-well injectionflowback and production tests, Geothermics, doi:10.1016/j.geothermics.2016.01.015

Ghergut, I., Behrens, H., Maier, F., Karmakar, S., Sauter, M., 2011. A note about "heat exchange areas" as a target parameter for SWIW tracer tests. In: Proceedings of the 36th Workshop on Geothermal Reservoir Engineering, Stanford University, Stanford, California. January 31 February 2, 2011, SGP-TR-191.

Ghergut, I., Behrens, H., Sauter, M., 2013a. Can Peclet numbers depend on tracer species? Going beyond SW test insensitivity to advection or equilibrium exchange. In: Proceedings 38th Workshop on Geothermal Reservoir Engineering, Stanford Univ. (CA), SGP-TR-198, 326335 .

Ghergut, I., Behrens, H., Sauter, M., 2013b. Single-well tracer push-pull test sensitivity to fracture aperture and spacing. In: Proceedings 38th Workshop on Geothermal Reservoir Engineering, Stanford Univ. (CA), SGP-TR-198, 295-308.

Haluszczak, L.O., Rose, A.W., Kump, L.R., 2013, Geochemical evaluation of flowback brine from Mar- cellus gas wells in Pennsylvania, USA, Applied Geochemistry, J. 28, 55-61, http:// dx.doi.org/10.1016/j.apgeochem.2012.10.002.

Jackson, R. B., Vengosh, A., Darrah, T. H., Warner, N. R., Down, A., Poreda, R. J., Osborn, S. G., Zhao, K., Karr, J. D., 2013. Increased Stray Gas Abundance in a Subset of Drinking Water Wells Near Marcellus Shale Gas Extraction. Proc. Natl. Acad. Sci. USA 110 (28), 1125011255

Jung, Y., Pruess, K., 2011. A closed-form analytical solution for thermal single-well injectionwithdrawal tests. Water Resources Research 48(3), DOI: 10.1029/2011WR010979.

Karmakar, S., Ghergut, I., Sauter, M., 2015a. Early-flowback tracer signals to induced-fracture characterization in crystalline and sedimentary formation-a parametric study. Geothermics, 63. doi:10.1016/j.geothermics.2015.08.007

Karmakar, S., Ghergut, I., Sauter, M., 2015b. EGS in sedimentary basins: sensitivity of earlyflowback tracer signals to induced-fracture parameters. Energy Procedia 76, 223-229. doi:10.1016/j.egypro.2015.07.906

King, G. E., 2012, Hydraulic Fracturing: What every representative, environmentalist, regulator, reporter investor, university researcher, neighbor and engineer should know about estimating frac risk and improving frac performance in unconventional gas and oil wells, SPE 152596, SPE Hydraulic Fracturing Technology Conference, Woodlands, Texas, USA, February 2012.

Kocabas, I., Horne, R.N., 1987. Analysis of injection-backflow tracer tests in fractured geothermal reservoirs. In: Proceedings of the 12th Workshop on Geothermal Reservoir Engineering, Stanford University, Stanford, California, January 20-22, 1987, SGP-TR-109

Lan, Q., Ghanbari, E., Dehghanpour, H., Hawkes, R., 2014, Water loss versus soaking time: Spontaneous imbibition in tight rocks, SPE 167713, The SPE/EAGE European Unconventional Conference and Exhibition, Vienna, Austria, 25-27 February, 2014-a. 
Makhanov, K., Habibi, A., Dehghanpour, H., Kuru, E., 2014, Liquid uptake of gas shales: A workflow to estimate water loss during shut-in periods after fracturing operations, Journal of Unconventional Oil and Gas Resources 7, 22-32.

Ning, X., Fan, J., Holditch, S.A., and Lee, W.J., 1993, The Measurement of Matrix and Fracture Properties in Naturally Fractured Cores, SPE-25898, Low Permeability Reservoirs Symposium, Denver, Colorado, 26-28 April 1993.

Pruess, K., Doughty, C., 2010. Thermal single-well injection-withdrawal tracer tests for determining fracture-matrix heat transfer area. In: Proceedings 35th Workshop on Geothermal Reservoir Engineering, Stanford University (CA), SGP-TR-188.

Rose, P.E., Benoit, W.R., and Kilbourn, P.M. 2001, The application of the polyaromatic sulfonates as tracers in geothermal reservoirs: Geothermics, 30(6), pp. 617-640.

Rose P.E., Riasetto D, Siy J, Bart M, Reimus P, Mella M, Leecaster K, and Petty S., 2011, Quantum dots as tracers in geothermal and EGS reservoirs, PROCEEDINGS, Thirty-Sixth Workshop on Geothermal Reservoir Engineering Stanford University, Stanford, California, January 31 February 2, 2011SGP-TR-191

Rose, P., Leecaster, K., Clausen, S., Sanjuan, R., Ames, M., Reimus, P., Williams, M., Vermeul, V., Benoit, D., 2012, A Tracer Test at the Soda Lake, Nevada Geothermal Field Using a Sorbing Tracer. In: Proceedings $37^{\text {th }}$ Workshop on Geothermal Reservoir Engineering, Jan. 30 - Feb. 1, 2012, Stanford Univ. (CA), SGP-TR-194.

Rimassa, S.M., Howard, P.R., Blow, K.A., 2009, Optimizing fracturing Fluids from Flowback Water, SPE Tight Gas Completions Conference, San Antonio, Texas, USA, 15-17 June 2009.

Schroth, M.H., Istok, J.D., Haggerty, R., 2001. In situ evaluation of solute retardation using singlewell push-pull tests. Advances in Water Resources, 24, 105-117.

Tester, J. W., Anderson, B. J., Batchelor, A. S., Blackwell, D. D., DiPippo, R., Drake, E. M., Garnish, J., Livesay, B., Moore, M. C., Nochols, K., Petty, S., Toks• oz, M. N., and Veatch, R. W. J. 2006. The Future of GeothermalEnergy: Impact of Enhanced Geothermal Systems (EGS) on the United States in the 21st Century. Massachusetts Institute of Technology, Idaho Falls.

Vengosh, A., Jackson, R. B., Warner, N., Darrah, T. H., Kondash, A., 2014. A critical review of the risks to water resources from unconventional shale gas development and hydraulic fracturing in the United States. Environ. Sci. Technol. 48, $8334-8348$

Zolfaghari, A., Noel, M., Dehghanpour, H., Bearinger, D., 2014 Understanding the Origin of Flowback Salts: A Laboratory and Field Study, SPE-171647-MS, SPE/CSUR Unconventional Resources Conference-Canada, Calgary, Alberta, Canada, 30 September-2 October 2014.

Zolfaghari, A., Dehghanpour, H., Bearinger, D., 2015, Fracture Characterization using Flowback Salt- Concentration Transient, SPE Journal, J168598, DOI: 10.2118/168598-PA. 
Appendix 1-Early flowback tracer signal for fracture characterization using proppant sorptive, matrix sorptive and conservative tracers

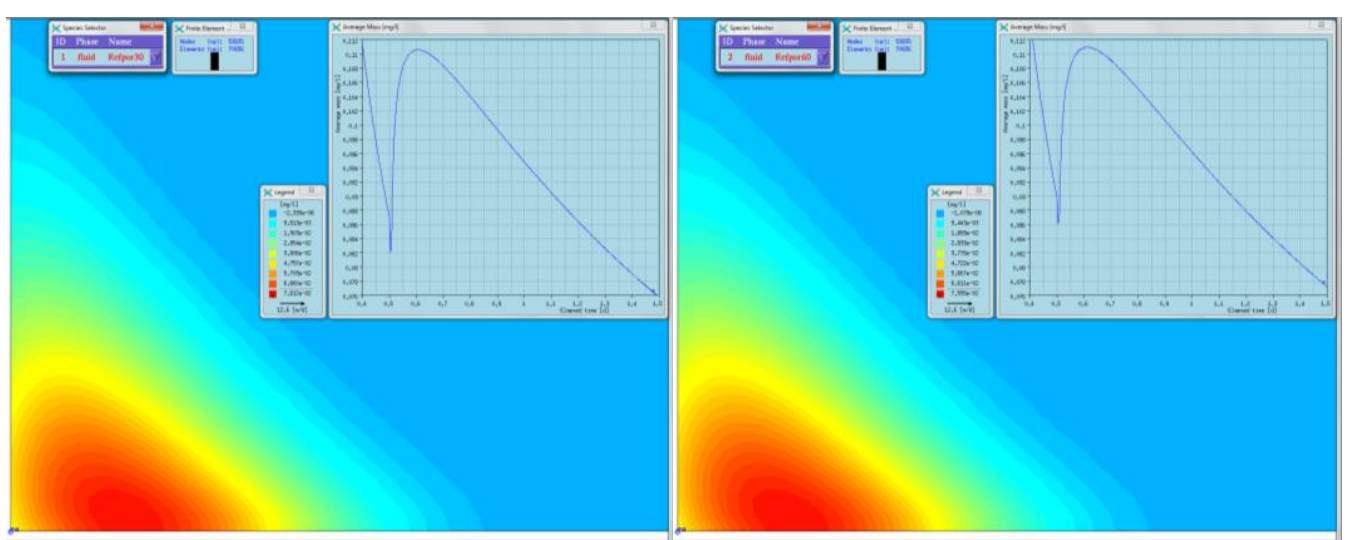

Figure 1.1: Conservative tracer spreading at the end of $V_{\text {inj }} V_{\text {ext }}=1$ time during early flowback test a) fracture porosity $30 \%$, b) fracture porosity $60 \%$
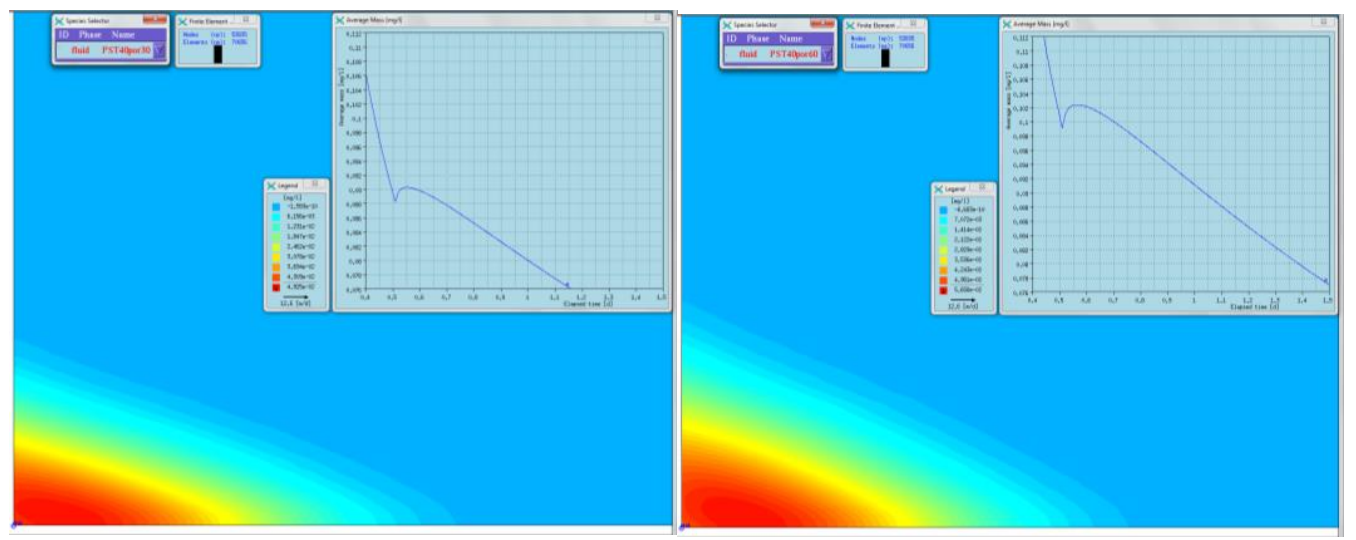

Figure 1.2: Proppant sorptive tracer spreading at the end of $V_{\text {inj }} / V_{\text {ext }}=1$ time during SW early flowback test a) fracture porosity $30 \%$, b) fracture porosity $60 \%$
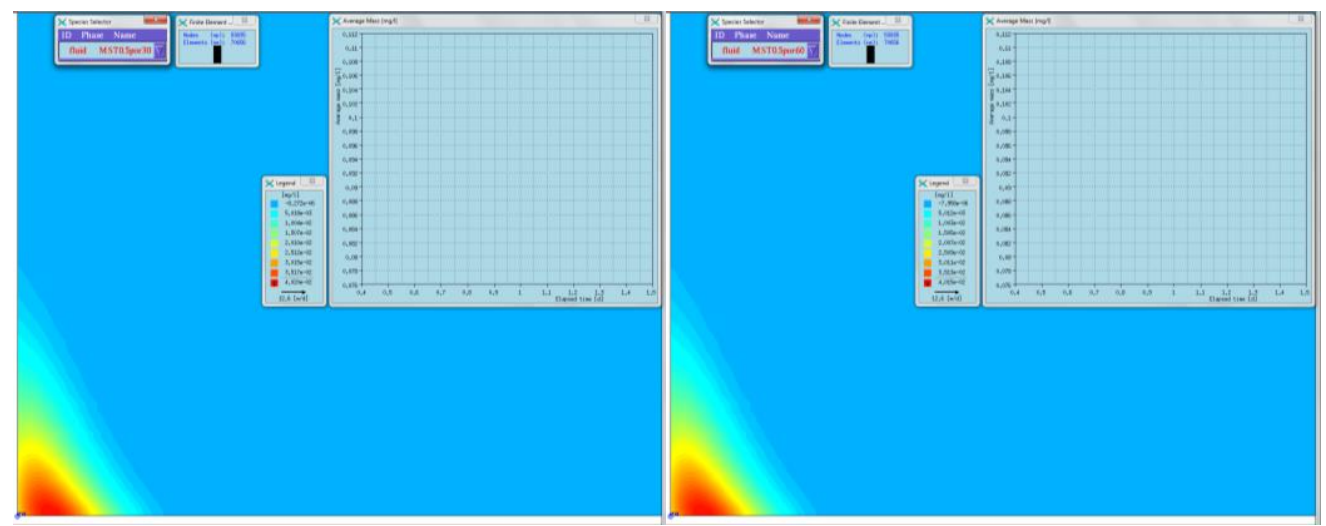

Figure 1.3: Matrix sorptive tracer $(R, k=0.5)$ spreading at the end of $V_{\text {inj }} / V_{\text {ext }}=1$ time during $S W$ early flowback test a) fracture porosity $30 \%$, b) fracture porosity $60 \%$ 


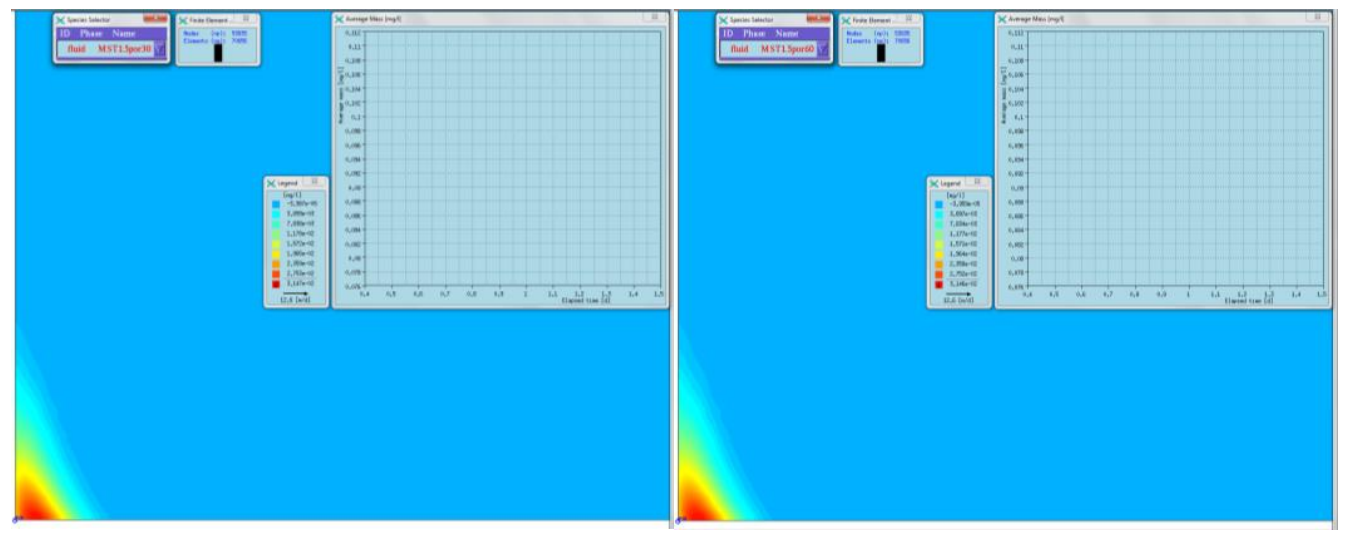

Figure 1.4: Matrix sorptive tracer $(R, k=1.5)$ spreading at the end of $V_{\text {inj }} / V_{\text {ext }}=1$ time during SW early flowback test a) fracture porosity $30 \%$, b) fracture porosity $60 \%$ 
Appendix 2a: Single-well tracer injection-flowback test simulation in fractured georeservoir in FEFLOW and COMSOL
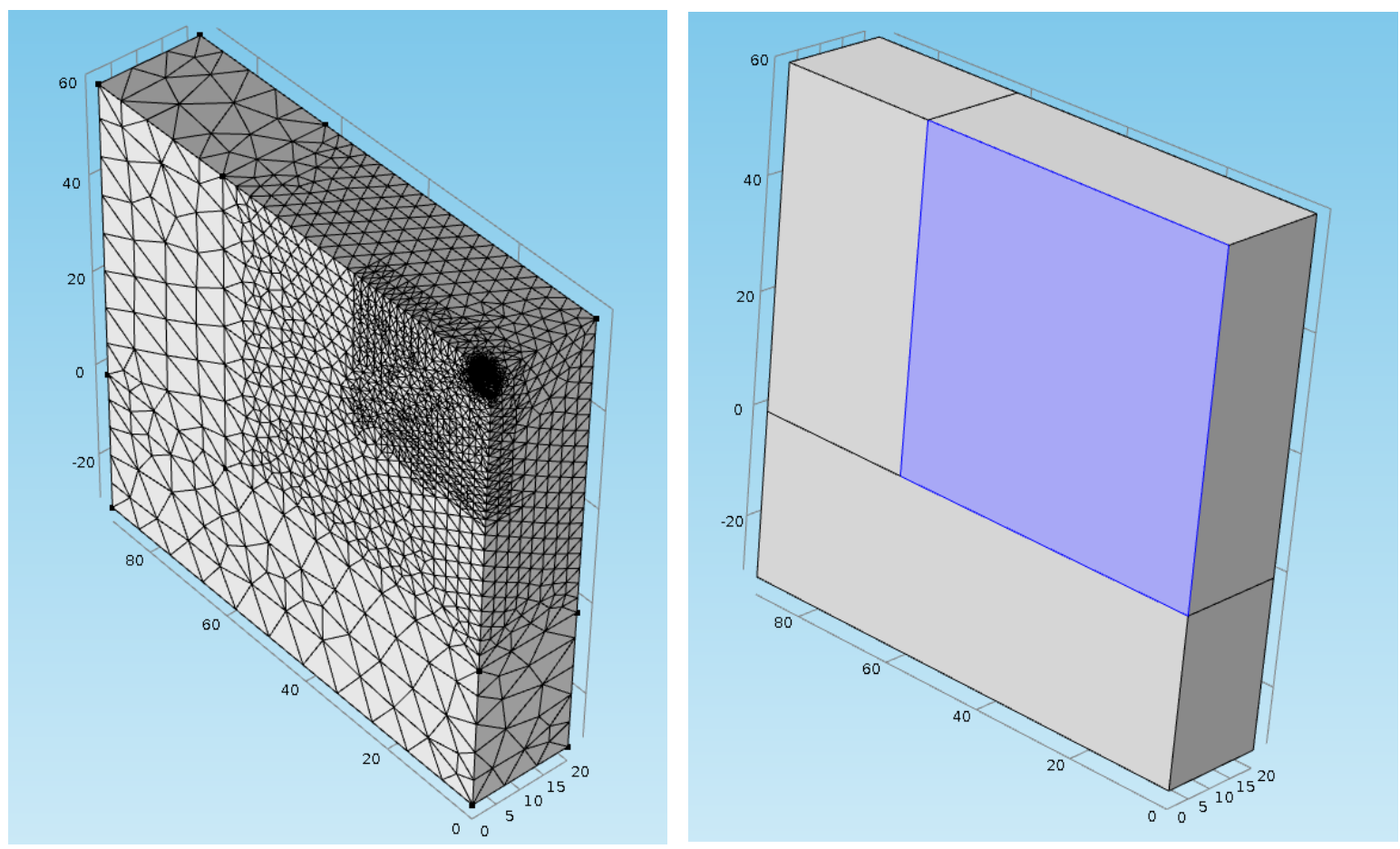

Figure 2a.1: Mesh refinement at the push-pull well for injection-flowback benchmark study in single fracture-matrix domain using PDEs module in software package COMSOL Multiphysics.
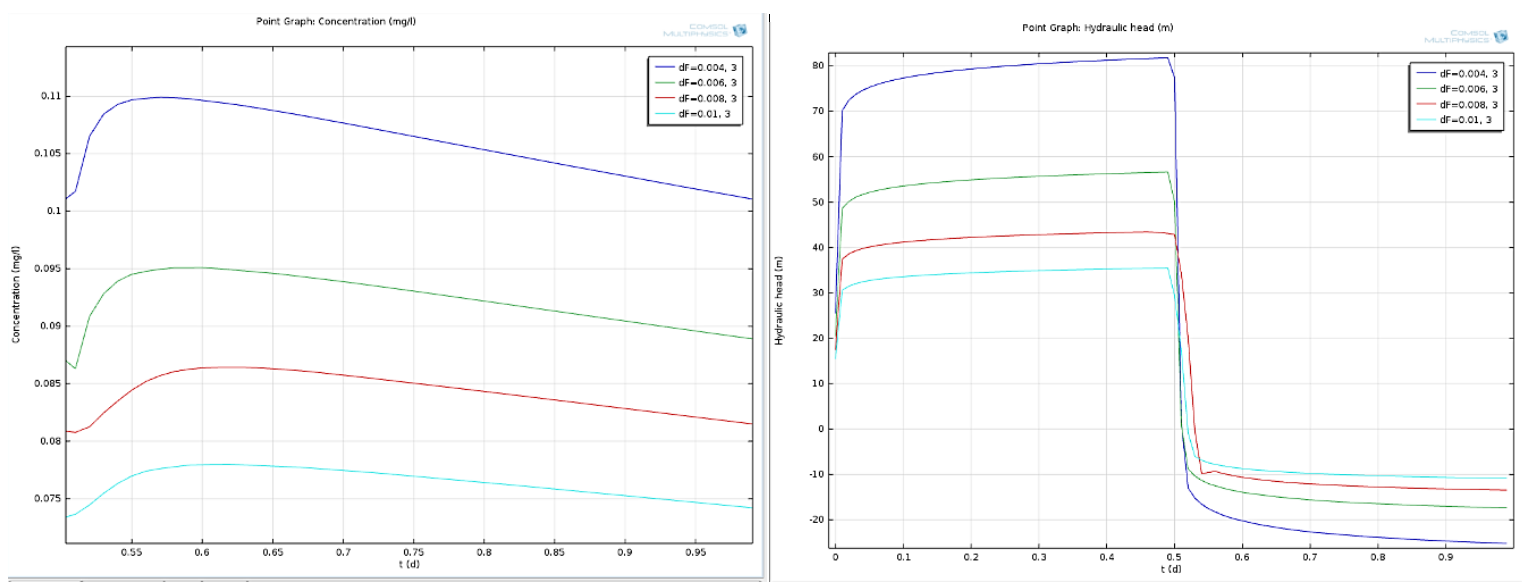

Figure 2a.2a, b: Tracer concentration and hydraulic head from different fracture thickness for injectionflowback benchmark study in single fracture-matrix domain using PDEs module in software package COMSOL Multiphysics. 


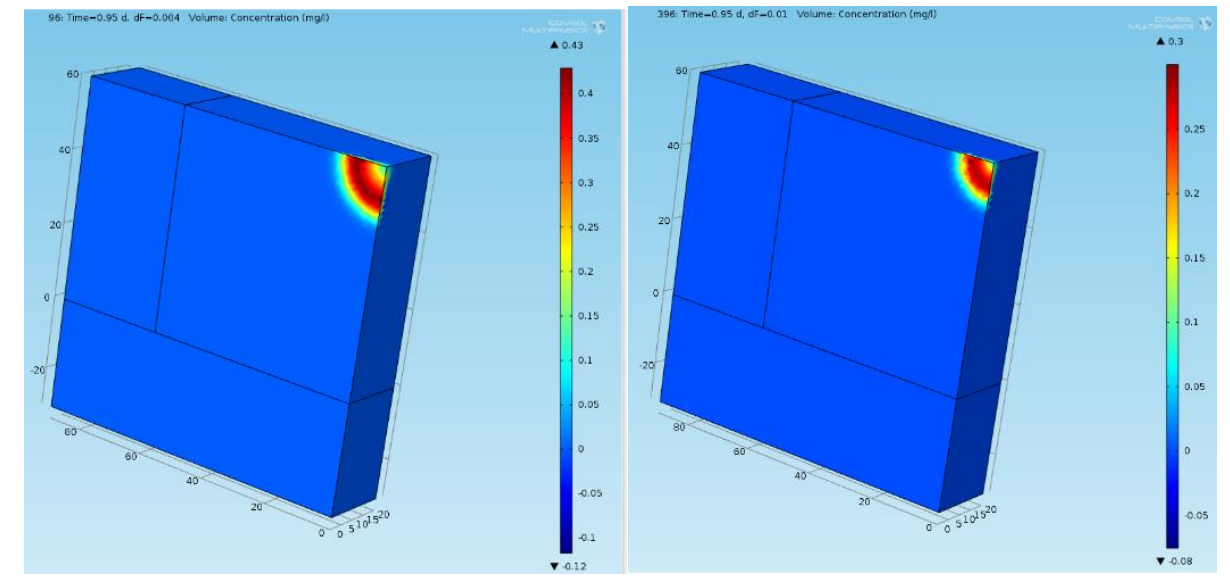

Figure 2a.3a, b: Tracer concentration from different fracture thickness (8mm-left, 20mm-right) for injection-flowback benchmark study in single fracture-matrix domain using PDEs module in software package COMSOL Multiphysics. 


\section{Appendix 2b: A set of benchmark studies for flow and solute transport in geo-reservoir}

Problem 2: 2D Dispersivity sensitivity study in different software with a mesh $40 \times 80$

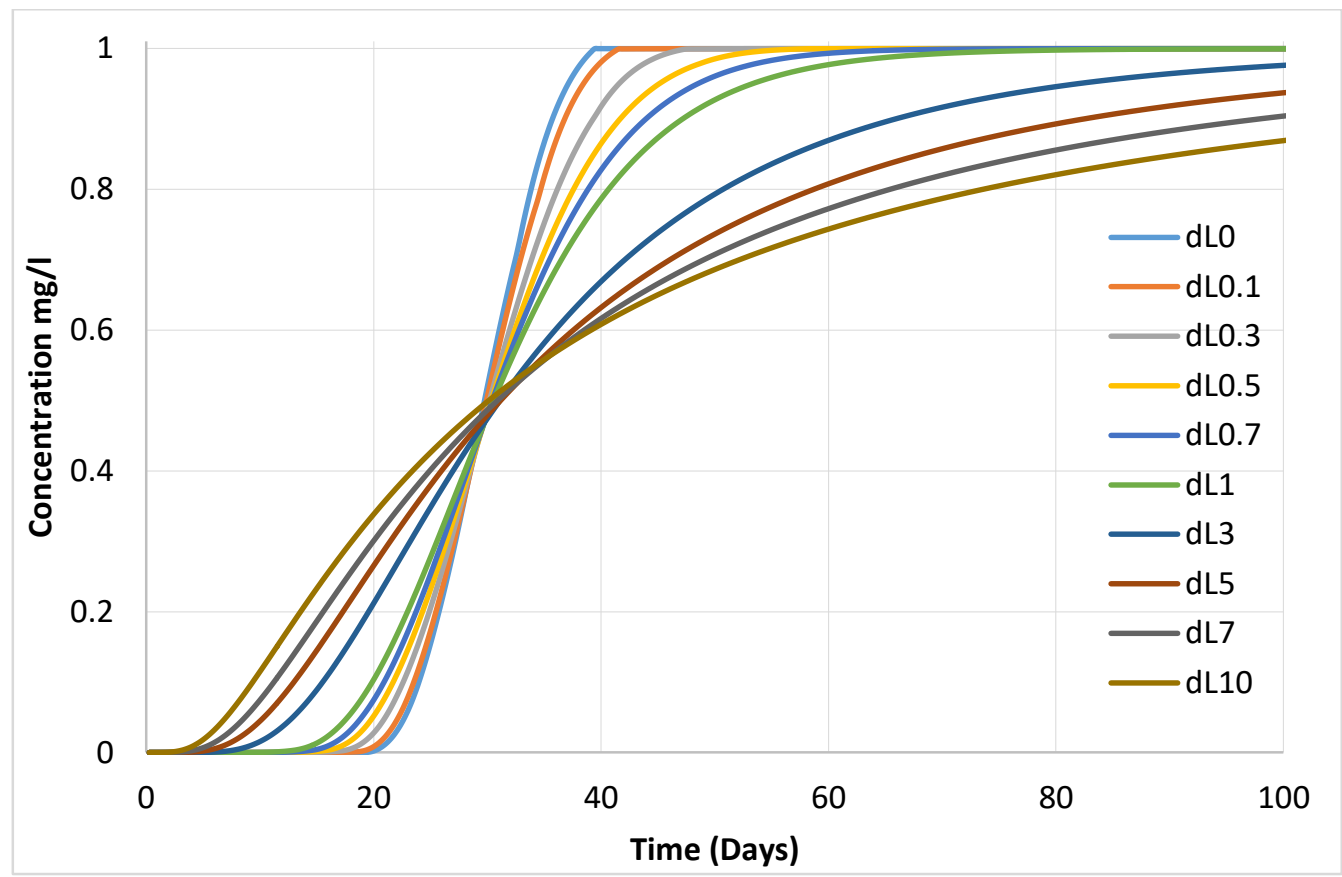

Figure 2.1: MF/MT3DMS simulation concentration results for various longitudinal dispersivity, where transversal dispersivity is $1 / 10$ of longitudinal dispersivity for a constant input concentration $1 \mathrm{mg} / \mathrm{l}$ in the fluid flow of $17.28 \mathrm{~m} 3 /$ day

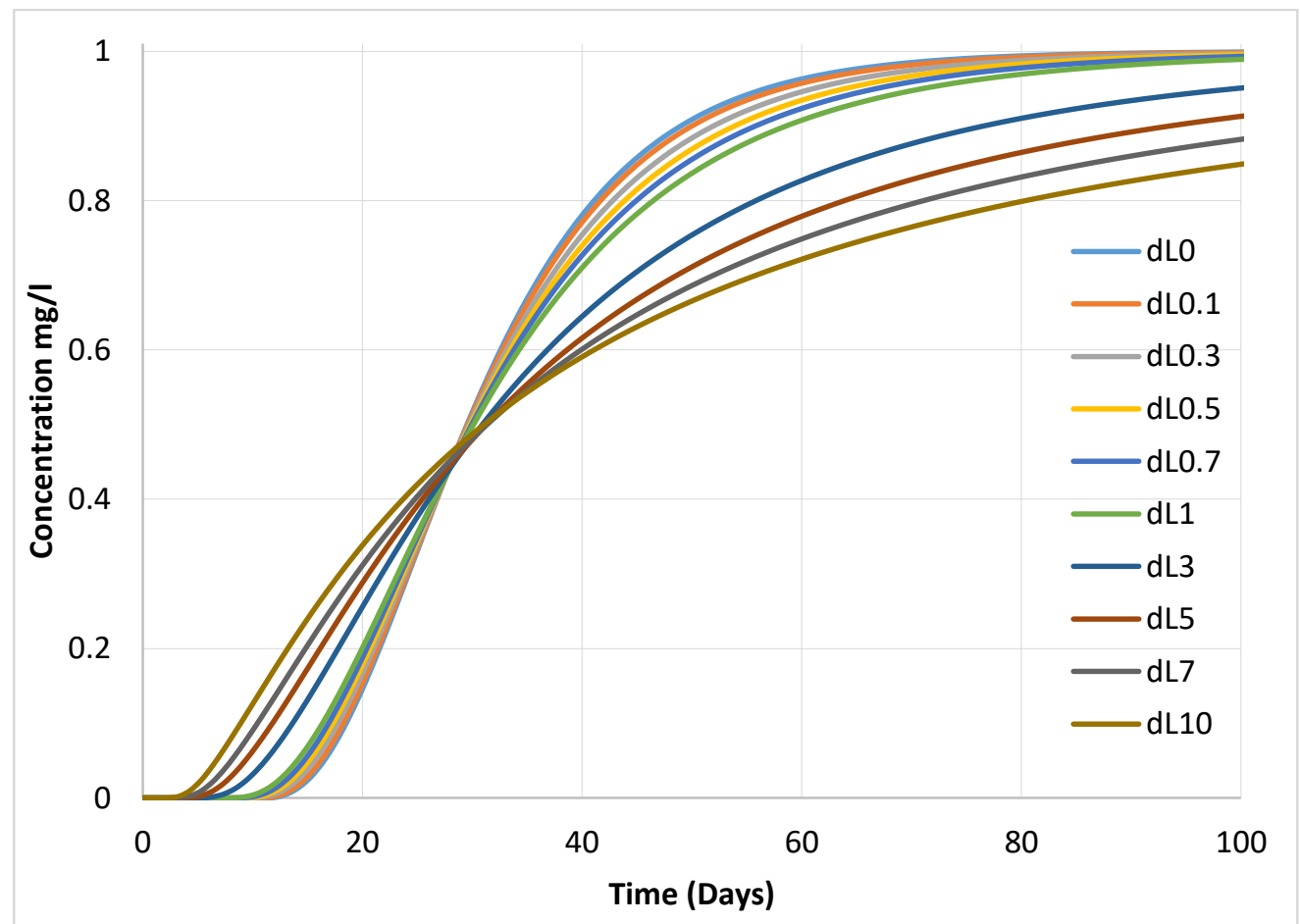

Figure 2.1: Problem 2:2D, FEFLOW simulation concentration results using using Full Upwinding method for different longitudinal dispersivity, where transversal dispersivity is $1 / 10$ of longitudinal dispersivity 
for a constant input concentration $1 \mathrm{mg} / \mathrm{l}$ in the fluid flow of $17.28 \mathrm{m3} /$ day. FEFLOWsimulation result shows smooth tracer simulation curves wheres ! shows strong numerical dispersion!

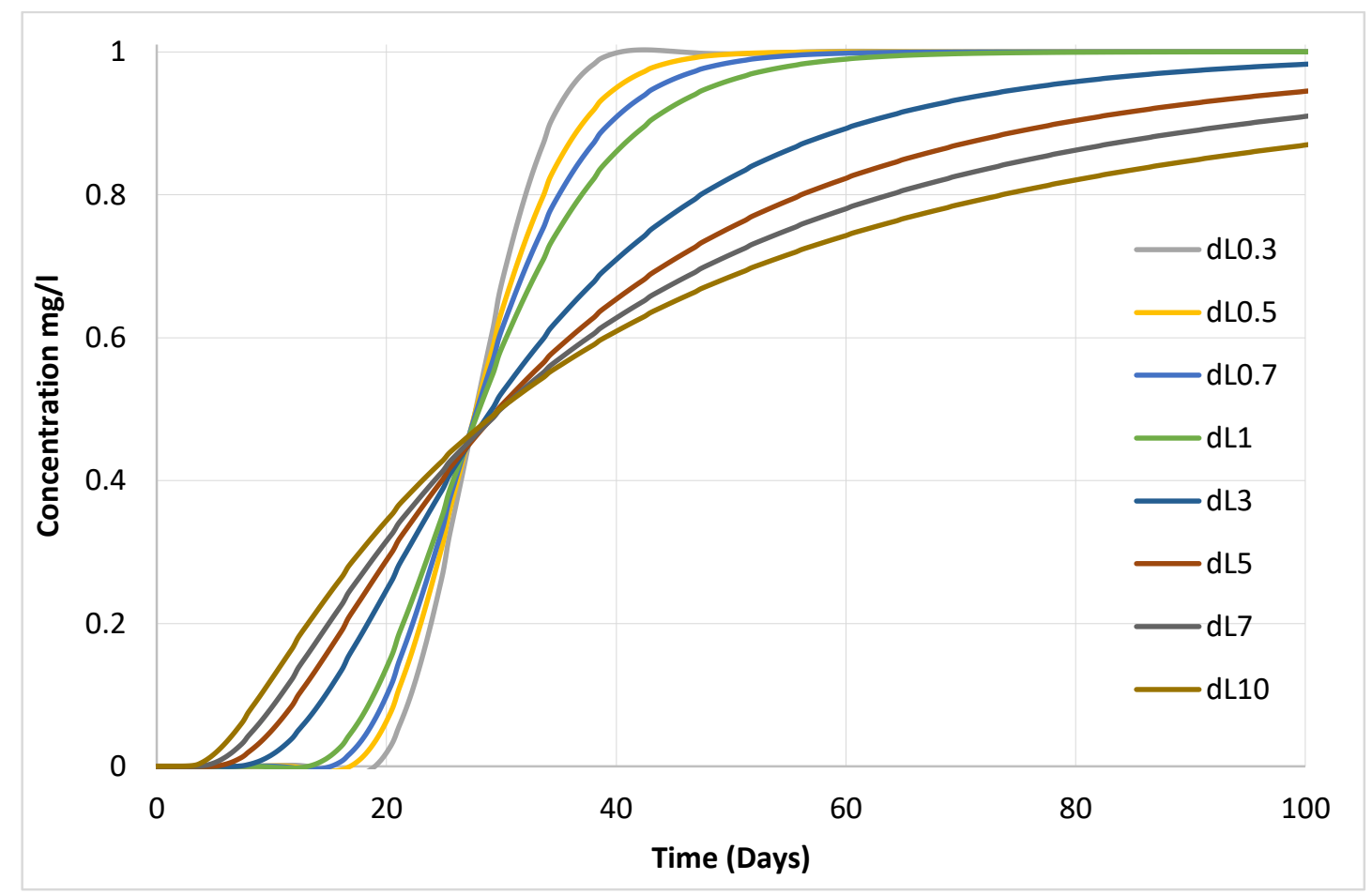

Figure 2.2: FEFLOW simulation concentration results using FEM Galerkin method for different longitudinal dispersivity, where transversal dispersivity is $1 / 10$ of longitudinal dispersivity for a constant input concentration $1 \mathrm{mg} / \mathrm{l}$ in the fluid flow of $17.28 \mathrm{~m} 3 /$ day

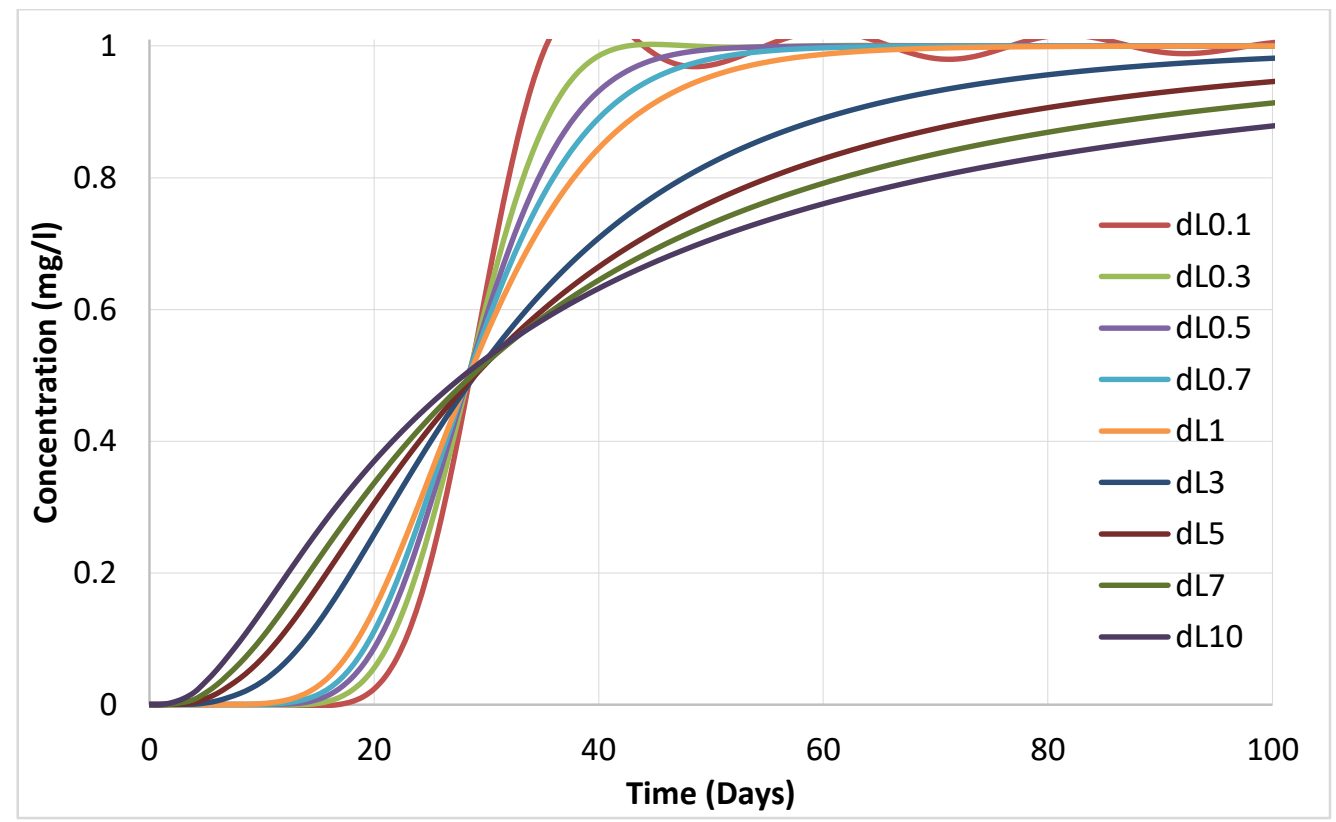

Figure 2.3: COMSOL simulation concentration results for different longitudinal dispersivity, where transversal dispersivity is $1 / 10$ of longitudinal dispersivity for a constant input concentration $1 \mathrm{mg} / \mathrm{l}$ in the fluid flow of $17.28 \mathrm{~m} 3 /$ day 


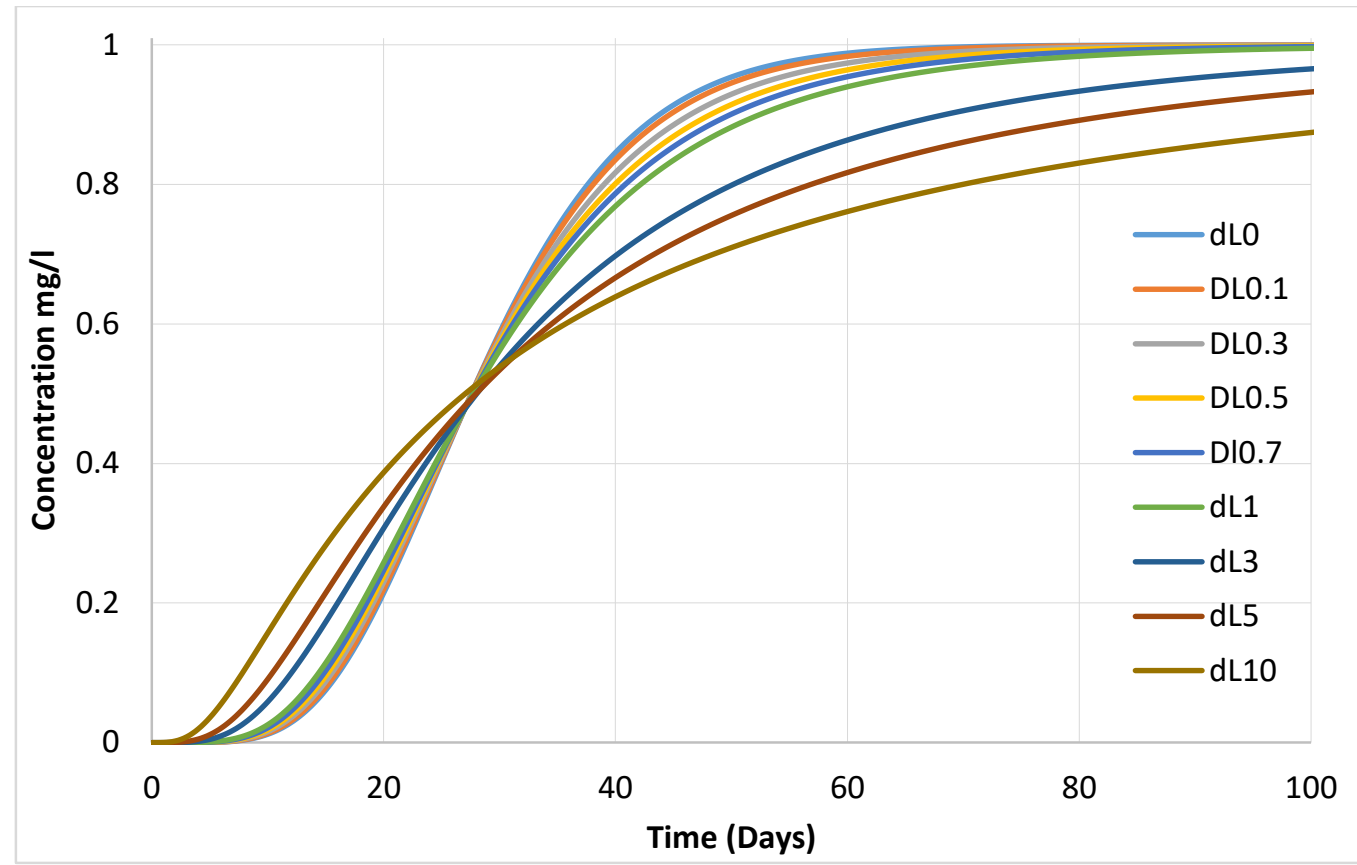

Figure 2.4: $D U M U^{x}$ simulation concentration results for different longitudinal dispersivity, where transversal dispersivity is $1 / 10$ of longitudinal dispersivity for a constant input concentration $1 \mathrm{mg} / \mathrm{l}$ in the fluid flow of $17.28 \mathrm{~m} 3 /$ day

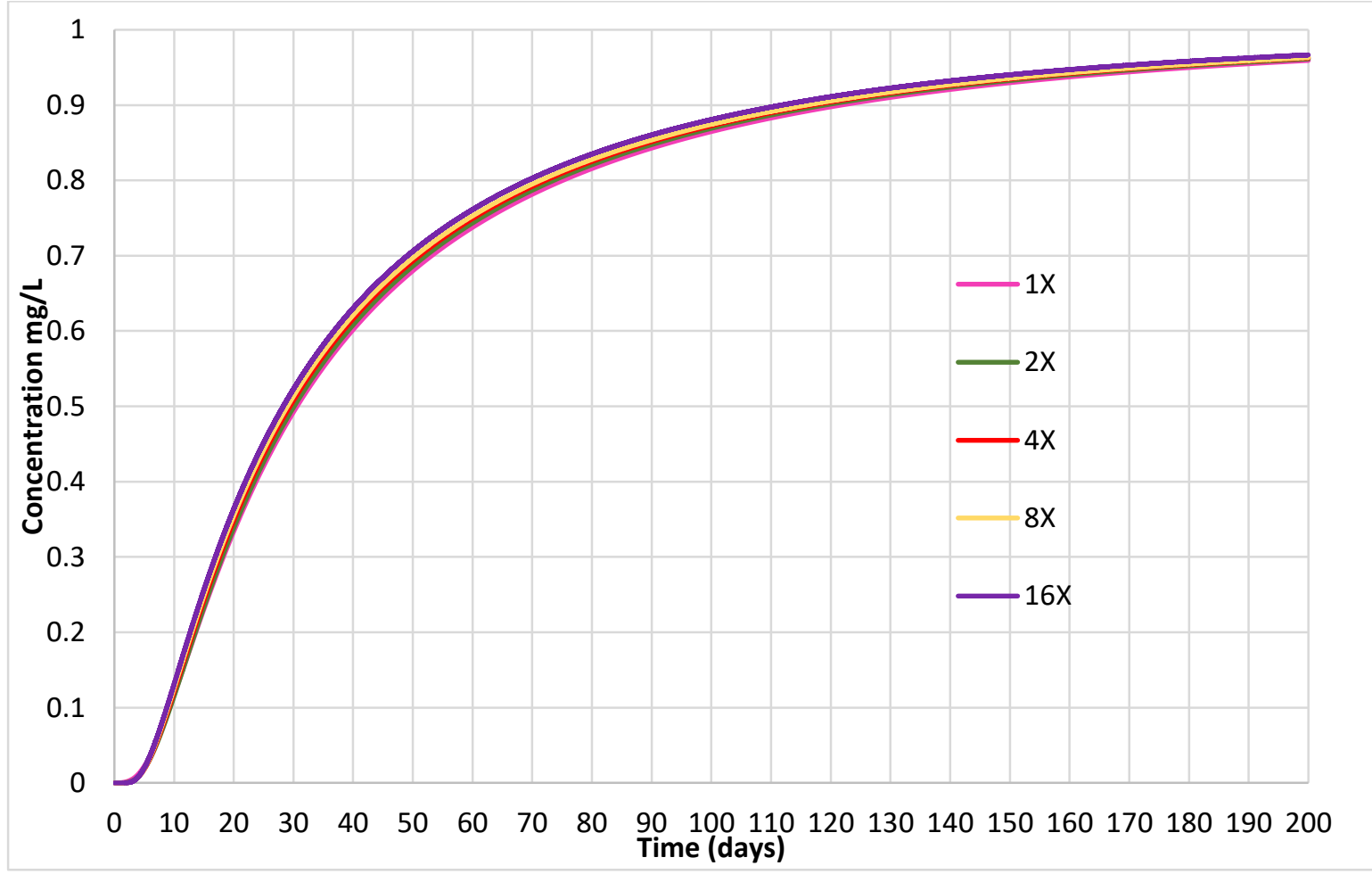

Figure 2.5: MF/MT3DMS simulation concentration results for different discretization, where transversal dispersivity is $1 / 10$ of longitudinal dispersivity for a constant input concentration $1 \mathrm{mg} / \mathrm{l}$ in the fluid flow of $17.28 \mathrm{~m} 3 /$ day 
Problem 3: 3D Dispersivity sensitivity study in different software with a mesh $20 \times 40 \times 24$

Hydraulic Head in different software
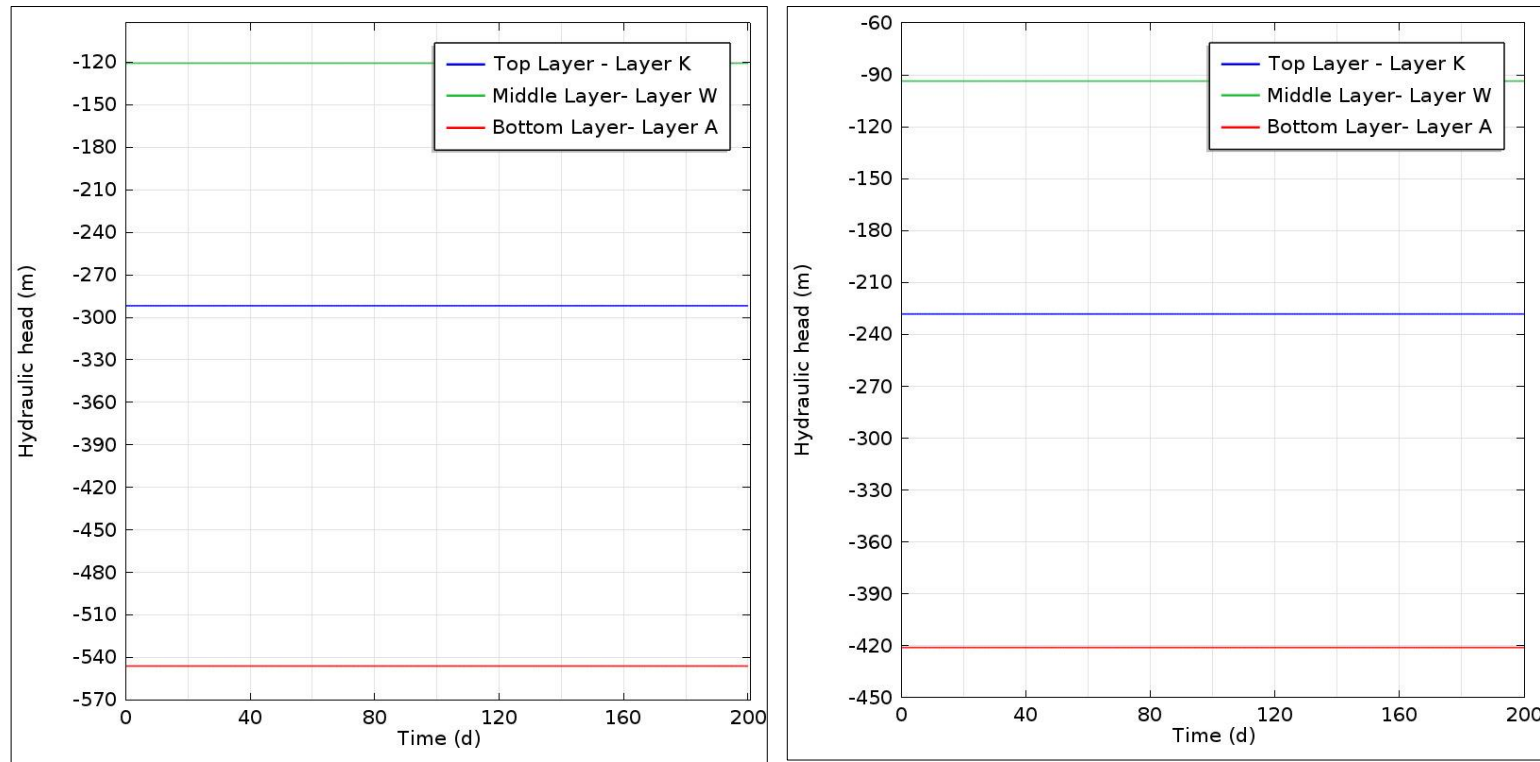

Figure 2.6: Problem 3, 3D-hydraulic head simulation result in COMSOLfor three layers, left figure shows the result from sampling point located at the top of the layer, and right figure is from sampling point at the middle of the layer. i.e mean head

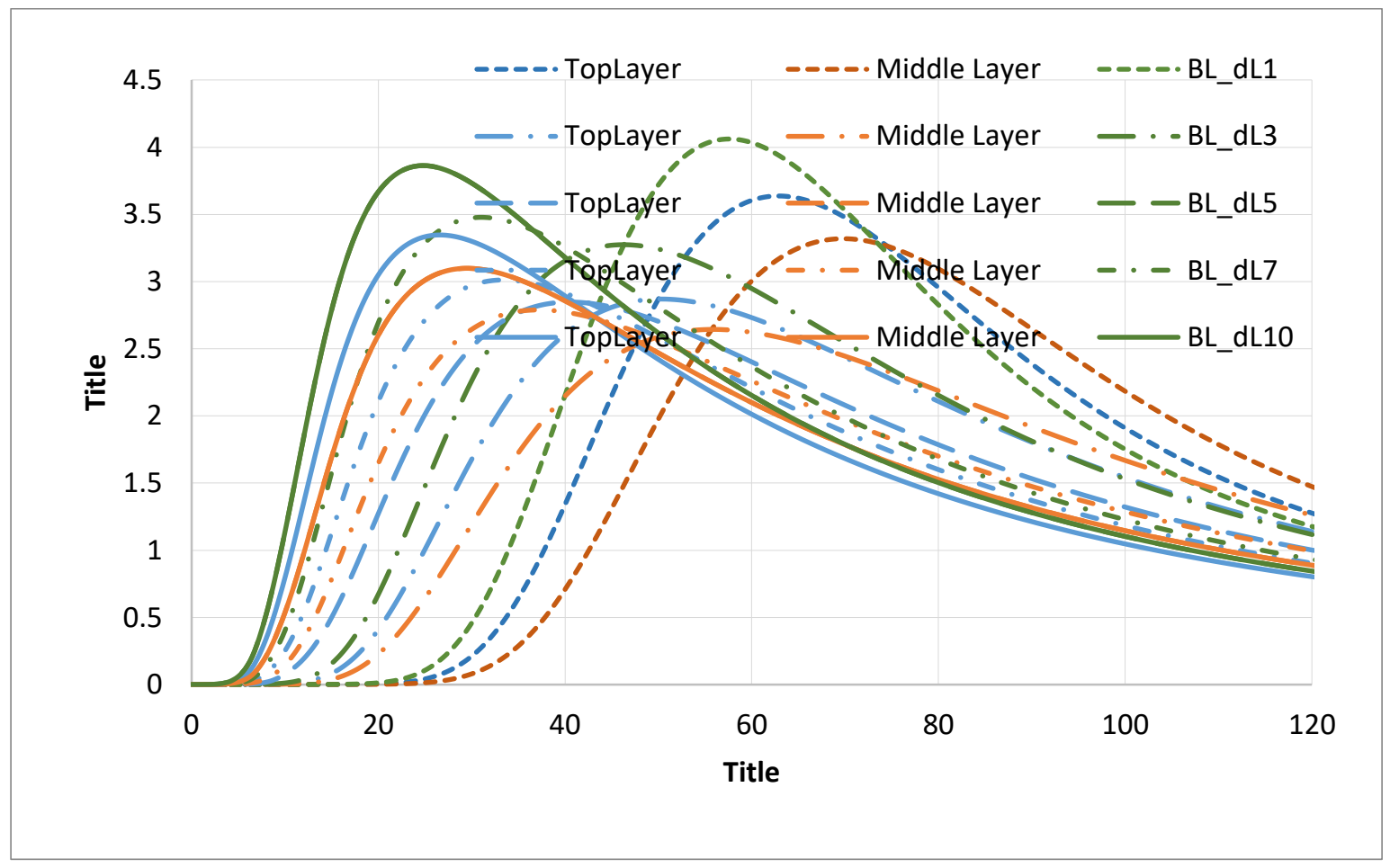


Figure 2.7: Dispersion sensitivity of tracer concentration simulated using MF/MT3DMS sampling at top of the layer (a higher pressure than the mean pressure across the layer)

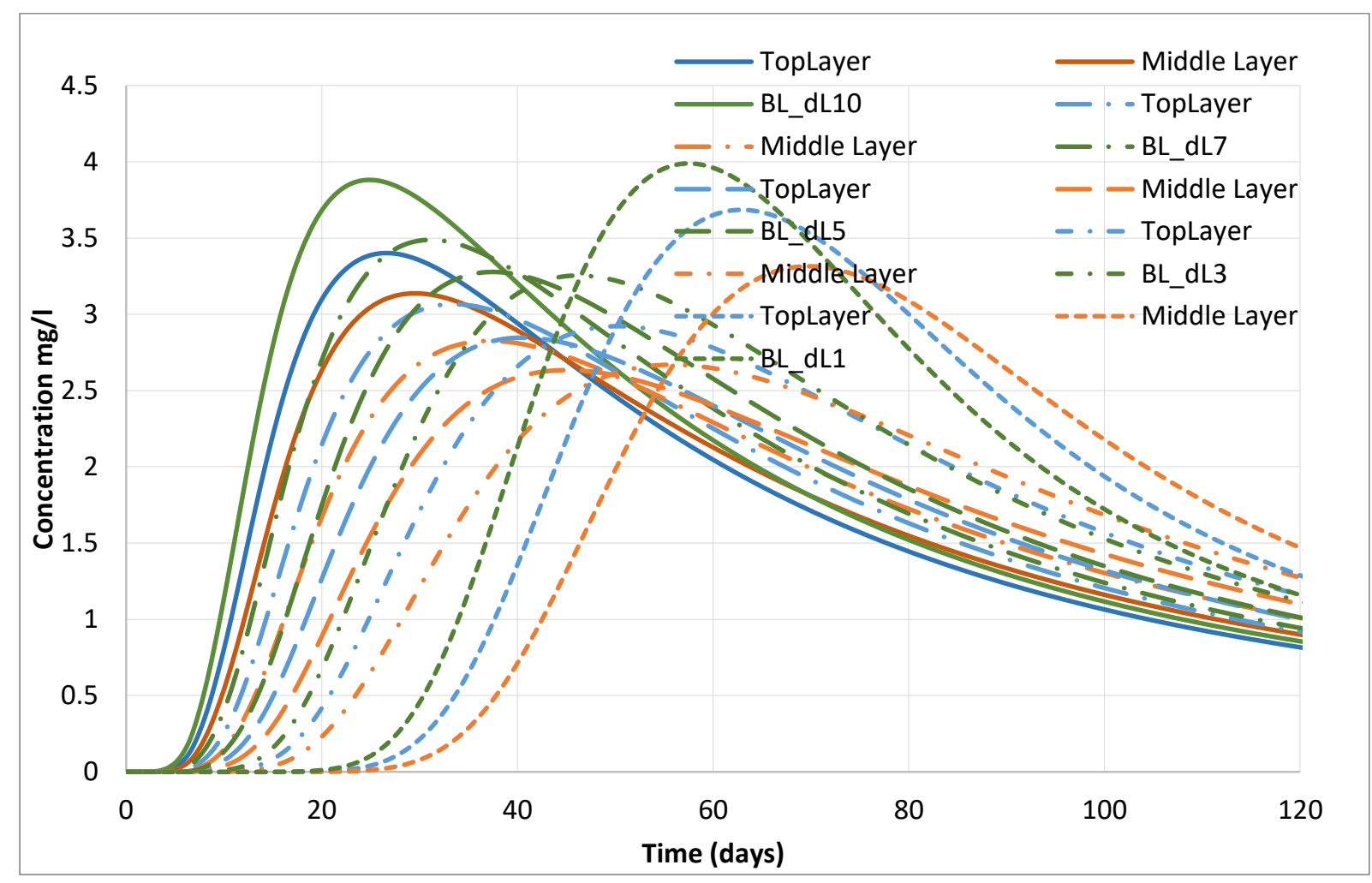

Figure 2.8: Dispersion sensitivity of tracer concentration simulated using MF/MT3DMS, sampling at middle of the layer (assuming a mean pressure across the layer)

Comsol UG results on 3D simulation for Helez

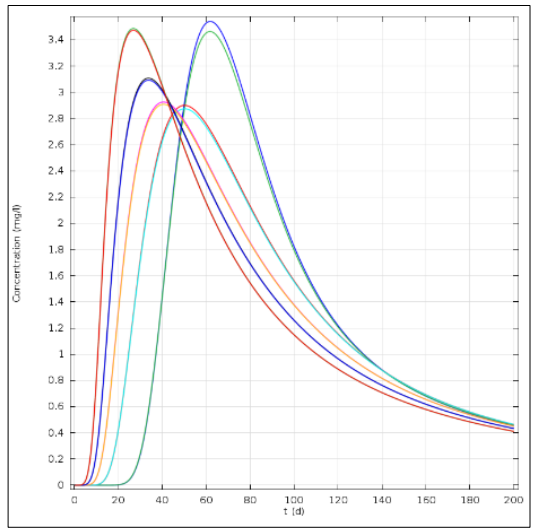

Top Layer

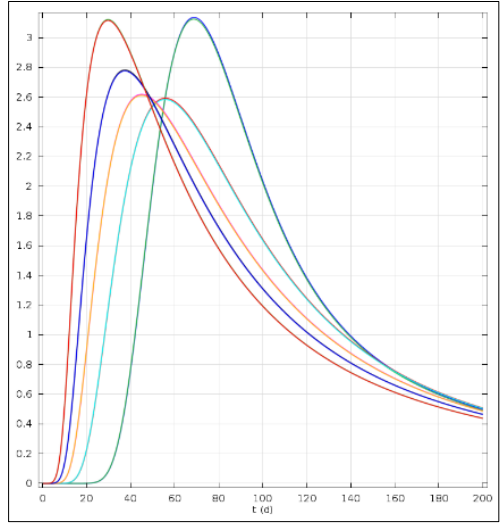

Middle layer

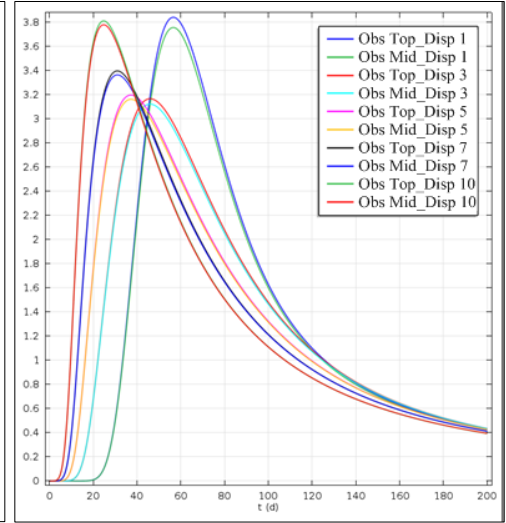

Bottom layer 
Figures 2.10: Each pair of curves represent tracer BT at the top and middle sampling point of the layer for a dispersivity value $1 \mathrm{~m} 3 \mathrm{~m}, 5 \mathrm{~m}, 7 \mathrm{~m}, 10 \mathrm{~m}$. A higher concentration curves show from top sampling point for each pair of curves
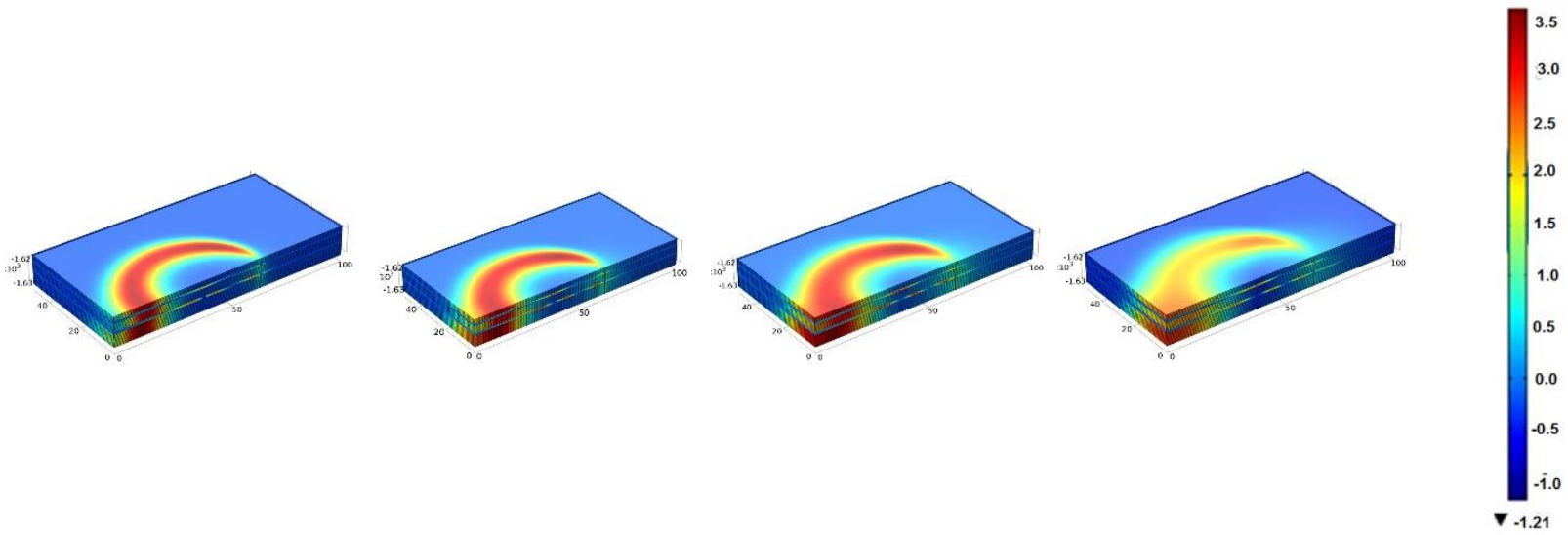

Figure 2.11: Problem 3, 3D-Simulation result on concentration spreading, at simulation time 200 days in COMSOLfor three the layers, the figure shows the result from dispersivity value $3 \mathrm{~m}, 5 \mathrm{~m}, 7 \mathrm{~m}$ and $10 \mathrm{~m}$ dispersivity value from left respectively. 


\section{List of Publications}

List of publications that are authored or co-authored by me-

1. Karmakar, S., Ghergut, J., Sauter, M., 2015. Early-flowback tracer signals for fracture characterization in an EGS developed in deep crystalline and sedimentary formations: a parametric study, Geothermics, in press (Special issue on EGS),

2. Karmakar, S., Ghergut, I., Sauter, M., 2015. EGS in sedimentary basins: sensitivity of early flowback tracer signals to induced-fracture parameters. Energy Procedia 76, 223 229.

3. Rahman M.A., Karmakar S., Salama H., Gactha-Bandjun N., Btatkeu K. B.D., Noubactep C. (2013): Optimising the design of Fe0-based filtration systems for water treatment: The suitability of porous iron composites. Journal of Applied Solution Chemistry and Modeling, 2, 165-177. DOI: http://dx.doi.org/10.6000/1929$\underline{5030.2013 .02 .03 .2}$

\section{Conference proceedings, poster, and oral presentation}

1. DGMK/ÖGEW Frühjahstagung in Celle on 22 and 23 April 2015)

2. Deutsche Geophysikalische Gesellschaft (DGG) 75. Jahrestagung on 24 March 2015 abstracts

3. Benchmark study on flow and solute transport in geological reservoirs, Shyamal Karmakar, Alexandru Tatomir, Sandra Oehlmann, Markus Giese, Martin Sauter "Computational Method in Water Resource on 10-13 June 2014 at the University of Stuttgart, Germany.”

4. Karmakar, S., Ghergut, I., Sauter, M., 2016, Early Time Flowback Test Tracer Selection for Stimulated Georeservoir Fracture Characterization. Stanford Geothermal Workshop, February 22-24

5. Co-operation or partial involvement with HEG M Sc. thesis work, During the Doctoral study period 10.2012-04.2016

i. Tolulope Abiodun Owonibi: Numerical benchmark study of single phase flow and transport in porous media (FEFLOW, COMSOL, and MODFLOW)

ii. Mr. Rizwan Mohsin: FSR study for Soulz-sous-Foret georeservoir and comparing translatability of parameter betweeen geo-reservoirs as well as carbonate aquifer. 
iii. Mr.Benjamin Olukunle Ekeade-Flow-storage repartitions (FSR) for tracer test evaluation and geothermal reservoir characterization- analyzing data and models of the Olkaria site in the East-Kenyan Rift Valley 


\section{Curriculum Vitae}

Shyamal Karmakar

PERSONAL DATA

Date of birth- 20.11.1980

Place of birth- Barkal, Rangamati

Nationality- Bangladeshi

\section{EDUCATION}

10/2012 - Current Georg-August-Universität Göttingen Geoscience Centre Department Applied Geology, Ph.D. Student

10/2009 - 11/2011 Georg-August-Universität Göttingen Geoscience Centre Department Applied Geology (degree: M Sc. in Hydrogeology and Environmental Geoscience)

02/2007 - 12/2008 Institute of Forestry and Environmental Sciences, University of Chittagong, M.S. in Environmental Science

01/2001 - 08/2006 Institute of Forestry and Environmental Sciences, University of Chittagong, B Sc. in Environmental Science

\section{WORK EXPERIENCE}

Since 05/2012 Lecturer, Institute of Forestry and Environmental Sciences, University of Chittagong, Bangladesh (On study leave since 08/2012)

02/2007- 09/2009 Institute of Forestry and Environmental Sciences, University of Chittagong, as research assistant 


\section{Acknowledgement}

My sincere thanks to my supervisors Professor Dr. Martin Sauter and Dr. Iulia Ghergut. Particularly like to mention Dr. Iulia Ghergut's extensive support throughout this period and help me to develop and process ideas, confront with my mistakes! My special thanks and gratitude to Professor Dr. Günter Buntebarth for his consent to assess my thesis as co-referee at the very last moment. My sincere thanks to all other members of the Examination Board, Professor Dr.-Ing. Thomas Ptak-Fix, Dr. Reinhard Jung and Dr. Ulrich Maier.

It has been a great occasion to work with Dr. Alex Tatomir and Dr. Sandra Oehlmann to create a fruitful project on benchmark study; thanks buddies. Thanks to all my colleagues in Applied Geology department, Ibrahim Hamadan, Markus Giese, Dr. Jannes Kordila, Dr. Torsten Lange, PD Dr. Chicgoua Noubactep, Dr. Sebastian Schmidt, Dr. Yulan Jin, Dr. William Allokhoury, Elena Shigorina, Peter Biermann, Anja Preglar, Dr. Matthias Franz, Mashia Mohammadfoghi, Viet Cao, Monthon and others. It has been a quality time with you in various occasions and discussion eventually does not limited to hydrogeology. Special thanks for Mrs. Beka PetersKewitz and Mr. Hartmut Düker.

I like to thank my home University, Chittagong University to grant me the study leave and keeping a place and opportunity to serve the country. I like to thank my teacher and colleagues Prof. Dr. Sirajul Haque, Prof. Dr. Tapan Kumar Nath, Prof. Dr. Mohammad Jashimuddin, Prof. Dr. Shafiul Alam, Tarit Kumar Bal and current director of the IFESCU Prof. Dr. Khaled Mizbahuzzaman and for their encouragement and support at many stages to avail this study leave and keep me motivated to accomplish my higher study. Thanks to my friends of Batch 22 and seniors of the institute who are always by my side in any needs of help.

I like to thank the small Bengali community, which has kept my memory and affection alive to the country.

Obviously my warmest thanks to my wife Mandira for her unconditional support and acceptance of my impatience and clumsy time. To my son Apratim (who has many complain about my disappearance during afternoon and evening time!), I hope you will forget this phase and forgive me, thanks for the sacrifice and understanding! My parents, especially my Mother, your inspiration, and sacrifice built me, kept me motivated at any stage of depression; as I know, the challenge you have faced and overcame, my struggle counts nothing to it! 
It is an excellent opportunity to achieve the finance and support from DAAD to continue this Ph.D. study. I should not forget the insurance, and administrative support from DAAD, also noteworthy to mention a nice opportunity to have a Deutschkurs in Leipzig. It is a great occasion for me to learn the language and culture also to live in Leipzig for four months. I do love to continue the journey in this language and culture! I promise. 